\begin{tabular}{|c|c|c|}
\hline Beitr. Ent. & Keltern & ISSN 0005-805X \\
\hline $\mathbf{5 3}(2003) 2$ & S. $341-435$ & 15.12 .2003 \\
\hline
\end{tabular}

\title{
Checklist of the Braconidae of Germany
}

\section{(Hymenoptera)}

\author{
S. A. Belokobylskij ${ }^{1}$, A. TAeger, C. van Achterberg, E. Haeselbarth, \\ M. RIEDEL
}

\section{Summary}

A revised checklist of the German braconids is presented. 1485 species are listed for Germany, accompanied by a reference. In addition doubtful records, species to be deleted from the German checklist, and species incertae sedis are listed. The dates of several important papers on braconids are discussed. Because these data and the comments in the list concern not only Germany, the main chapters were written in English.

\section{Zusammenfassung}

Es wird eine aktualisierte Checkliste der Braconidae Deutschlands vorgelegt. 1485 Arten werden, verbunden mit einer Quellenangabe, für Deutschland aufgeführt. Für zweifelhafte Nachweise sowie aus der Deutschlandliste zu streichende Arten und species incertae sedis erfolgte eine gesonderte Auflistung. Die Datierungen verschiedener wichtiger Arbeiten über Braconiden werden diskutiert. Weil diese Angaben und die Kommentare in der Liste in ihrer Wirkung über Deutschland hinausgehen, wurden die hauptsächlichen Kapitel auf Englisch verfasst.

\section{Contents / Inhalt}

Introduction 342

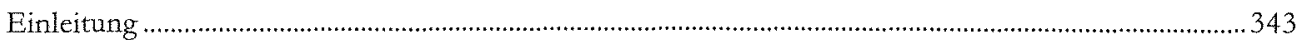

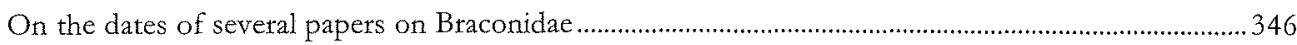

The dates of publication of NEES VON ESENBECK's "Ichneumonides adsciti ..." .........................346

PANZER's and HERRICH-SCHÄFFER's Braconidae in the "Faunae Insectorum Germanicae" ..............346

The dates of the Braconidae described by CurTts in the "British Entomology..." ............................348

The dates of publication of HALIDAY's papers on Braconidae .........................................................349

The dates of C. G. THOMSON's works on Braconidae ……................................................................352

The dates of publication of MARSHALl's volumes in "Species des Hyménoptères

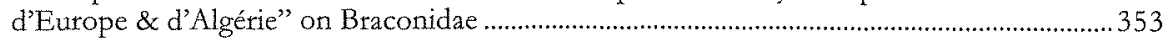

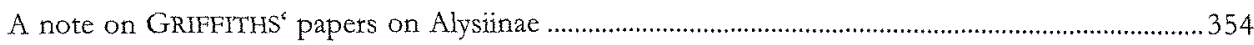

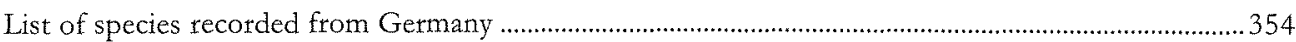

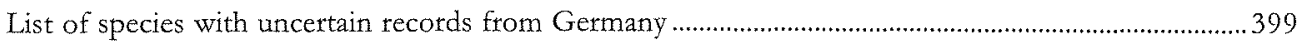

Species deleted from the German checklist ....................................................................................... 400

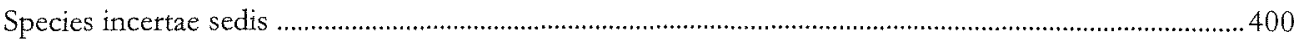

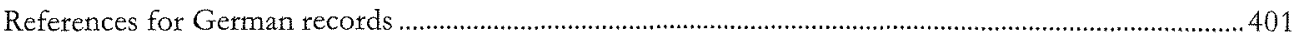

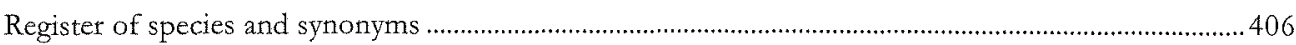

\footnotetext{
1 The work of S. A. BELOKOBYLSKIy was supported by the DFG grant 436 RUS $17 / 18 / 98$.
} 


\section{Introduction $\left(\mathrm{AT}^{2}\right)$}

The Braconidae is the second largest family of Hymenoptera in Germany after the Ichneumonidae. Although the family is rich in species, the group is also poorly known - this begins with the apparently trivial question of which species are even recorded in. Germany.

BELOKOBYLSKIJ \& TAEGER reported 1064 species from Germany in 2001 in the "Entomofauna Germanica"3. For various reasons this checklist remained very incomplete. To solve this problem, the two authors began the compilation of a revised list soon after the first was published. To further improve the quality and reliability of the list, C. VAN ACHTERBERG and E. HAESELBARTH were asked to assist. Both colleagues declared themselves willing, and their help enabled the presentation of the work in its present form.

Mr. VAN ACHTERBERG contributed previously unpublished information on synonymies of more than 30 nominal taxa. These are based mainly on unpublished revisions of the Opiinae, the Leiophron-complex, Eubazus, Syntretus and Aleiodes of the West Palaearctic. The reasons for these taxonomic-nomenclatural alternations are to appear soon in another publication. The inclusion here of this information should help to avoid future problems, in that a better nomenclatural agreement with the forthcoming "Fauna Europaea" (VAN ACHTERBERG, in prep.) is achieved.

Shortly, before the manuscript was completed, Mr. M. RIEDEL contacted us because he also was working on a more detailed, improved checklist of German Braconidae. From this work were taken valuable data on published German records.

The list presented includes not just the updated inventory of species, but also a source on which the record is based. An important disadvantage of "Entomofauna Germanica" is that although sources are listed under literature references, a direct link to the species is missing. Tracing the origin of the records is therefore made very difficult.

A considerable improvement over the old list is the inclusion of synonyms (including misidentifications and misspellings). The bibliographical references for these synonymies are not included, because these would have greatly exceeded the scope of the present work. The records of species listed below can be based on corresponding synonyms. We have let one source for each species suffice. These are listed with the following priority:

1. Material in museums (determined by recognised specialists). Extensive material was checked or determined in $1998 / 1999$ by S. BELOKOBYLSKIJ during a scientific visit funded by the DFG (436RUS17/18/98).

2. Literature record in a current revision of the group.

3. Literature record from a faunal list.

4. Literature record in the Hymenopterorum Catalogus (SHENEFELT, 1969-1978). This publication contains references to publications, which are comparable to 2. and 3 . above. We did not attempt to determine the corresponding original source.

\footnotetext{
${ }^{2}$ The responsibility for particular sections or commentaries is indicated by the following abbreviations: AT TAEger, CvA - VAN ACHTERBERG, EH - HAeselbarth, SB - BelokobylskiJ

${ }^{3}$ Belokobylski, S. A. \& TAEger, A. 2001: Braconidae. - In: DATHE, H. H.; TAEgER, A. \& BLANK, S. M. (Hrsg.): Verzeichnis der Hautflügler Deutschlands (Fauna Germanica 4). - Entomologische Nachrichten und Berichte, Dresden Beiheft 7: 103-115
} 
A further source of records, namely species (or their synonyms) with a type locality in Germany, is not separately dealt with. These taxa are in part covered by points 1 . and 2. (above). A total of approximately 600 species (or their synonyms) have been described from Germany. The bulk of these species were dealt with by the most important of the German specialists of the 19th Century: C. G. D. NEES VON ESENBECK (17761858), P. F. BOUCHÉ, (1783?-1856), J. F. RUTHE (1788-1859), G. A. W. HERRICHSCHÄFFer (1799-1874), J. T. C. RATzeburg (1801-1871), A. FörSTER (1810-1884) and H. REINHARD (1816-1892). With almost no exceptions, it can be taken that a taxon in this list described by these authors was based on German material.

Nomenclature and systematics follow the ideas of S. BELOKOBYLSKIJ. In a few cases, there are differing views as to the validity of individual species. Such controversial points are explained in corresponding commentaries. Generic placement often also differs between authors. Where it appeared necessary, and especially to promote "nomenclatural compatibility" with the immediately forthcoming "Fauna Europaea", alternative new combinations are referred to.

The literature contains frequent contradictions and unclarities in the dating of some taxa. For this reason the list is preceded by a section which deals with the main problems (see: On the dates of several papers on Braconidae).

As far as was possible, species were excluded from the list (see: Species deleted from the German checklist), which were recorded in error from Germany, or only in a historical context (outside the present borders). This type of exclusion is often difficult, because distributional information is not infrequently incorporated in subsequent publications without a reference. In such cases it is often impossible to decide whether an original record is involved or a record from previous literature.

For several different reasons, several records of species have to be considered as uncertain (see: Records from Germany uncertain).

It can be considered certain that a part of the species reported from Germany is based on misidentifications. On the other hand many of these species, on consideting their complete distribution, probably occur in Germany. As previously, many species are only known from neighbouring countries. Here also one must suspect a presence of the species in Germany. About 3,380 braconid species are now known in Europe (these and following numbers after VAN ACHTERBERG, in litt.). In the present list 1,485 species are considered to occur in Germany. If one accepts the species concepts of VAN ACHTERBERG, this number rises by about 10 .

Although the Netherlands (about 990 species) and Austria (about 760) have clearly less recorded species, so far approximately 300 of these species are missing from Germany. Considering these numbers, about 2000 species of Braconidae in surch not an overestimate for Germany.

\section{Einleitung $\left(\mathrm{AT}^{4}\right)$}

Die Brackwespen (Hymenoptera: Braconidae) sind nach den Schlupfwespen (Ichneumonidae) die artenreichste Hautflüglerfamilie in Deutschland. Diesem Artenreichtum steht

\footnotetext{
${ }^{4}$ Die Verantwortlichkeiten für bestimmte Abschnitte bzw. Kommentare wird durch die folgenden Abkürzungen gekennzeichnet: AT - TAEGER, CVA - VAN ACHTERBERG, EH - HAESELBARTH, SB - BELOKOBYLSKII
} 
allerdings ein geringes Wissen über die Gruppe gegenüber - und dies beginnt schon bei der scheinbar recht trivialen Frage, welche Arten in Deutschland überhaupt nachgewiesen sind.

BELOKOBYLSKIJ \& TAEGER meldeten 2001 in der „Entomofauna Germanica”5 1064 Arten aus Deutschland. Diese Checkliste ist leider aus verschiedenen Ursachen sehr lückenhaft geblieben. Um dieses Problem zu beheben, begannen die beiden Autoren unmittelbar nach der Publikation der erwähnten Liste, ein aktualisiertes Artenverzeichnis zusammenzustellen. Um die Qualität und Zuverlässigkeit der Liste weiter zu verbessern, wurden die Herren C. VAN ACHTERBERG und E. HAESELBARTH um Mitarbeit gebeten. Beide Kollegen erklärten sich hierzu bereit und halfen so, das neue Verzeichnis in der vorliegenden Form zu verfassen.

Herr VAN ACHTERBERG stellte bisher unveröffentlichte Informationen über Synonymien von mehr als 30 nominellen Taxa zur Verfügung. Diese basieren hauptsächlich auf unveröffentlichten Revisionen über Opiinae, den Leiopbron-Komplex, Eubazus, Syntretus und Aleiodes der Westpaläarktis. Die Begründungen der relevanten taxonomisch-nomenklatorischen Änderungen sollen demnächst an anderer Stelle erfolgen. Dieses soll Probleme in der Zukunft vermeiden, da damit eine bessere nomenklatorische Übereinstimmung mit der demnächst erscheinenden „Fauna Europaea” (VAN ACHTERBERG, in prep.) erreicht wird. Kurz vor Manuskriptabschluss setzte sich Hert M. RIEDEL mit uns in Verbindung, da er ebenfalls an einer verbesserten detaillierten Checkliste für die Braconidae Deutschlands arbeitete. Aus dieser Arbeit wurden wertvolle Hinweise auf publizierte deutsche Funde entnommen.

Diese hier vorgelegte Liste gibt, neben der Aufzählung der Arten, eine Quelle an, auf der die jeweilige Angabe basiert. Ein wesentlicher Nachteil der „Entomofauna Germanica" besteht darin, dass zwar Quellen im Literaturverzeichnis aufgelistet werden, doch ein direkter Bezug zu den Arten fehlt. Dadurch wird die Nachvollziehbarkeit der Angaben erheblich erschwert.

Eine wesentliche Verbesserung gegenüber der alten Liste besteht auch in der Auflistung der Synonyme (einschließlich Fehldeutungen und Fehlschreibungen). Auf bibliographische Belege für diese Synonymien wurde verzichtet, da diese den Rahmen der vorliegenden Arbeit gesprengt hätten. Die unten aufgeführten Nachweise der Arten können durchaus auf Meldungen der zugehörigen Synonyme beruhen. Wir haben uns mit jeweils einem Nachweis eines Taxon begnügt, wobei die Nachweise mit folgender Priorität verzeichnet sind:

1. Museumsmaterial (determiniert durch anerkannte Spezialisten). Umfangreiches Material wurde 1998/1999 von S. BELOKOBYLSKI im Rahmen des von der DFG geförderten Forschungsaufenthaltes (436 RUS 17/18/98) überprüft bzw. determiniert.

2. Literaturnachweis aus einer aktuellen Revision der Gruppe

3. Literaturnachweis durch faunistische Liste

\footnotetext{
"Betokobylskif, S. A. \& TAeger, A. 2001: Btaconidae. - In: DATHE, H. H.; TAEger, A. \& Blank, S. M. (Hrsg.): Verzeichnis der Hautflügler Deutschlands (Fauna Germanica 4). - Entomologische Nachrichten. und Berichte, Dresden Beiheft 7: 103-115
} 
4. Literaturnachweis im Hymenopterorum Catalogus (SHENEFELT, 1969-1978). Dieser Katalog enthält Verweise auf Veröffentlichungen, die mit 2. und 3. vergleichbar sind. Hier wurde von uns nicht versucht, die entsprechende Originalquelle zu ermitteln.

Eine andere Quelle für Nachweise, nämlich Arten (oder deren Synonyme) mit locus typicus in Deutschland, wurde nicht separat erfasst. Teilweise sind diese Taxa durch die Punkte 1. und 2. mit berücksichtigt. Insgesamt wurden ca. 600 Arten (bzw. deren Synonyme) aus Deutschland beschrieben. Die Masse dieser Arten geht auf die wichtigsten deutschen Spezialisten des 19. Jahrhunderts zurïck: C. G. D. NEES VON EsENBECK (1776-1858), P. F. BOUCHÉ, (1783?-1856), J. F. RUTHE (1788-1859), G. A. W. HERrICHSCHÄFFER (1799-1874), J. T. C. RATZEBURG (1801-1871), A. FÖRSTER (1810-1884) und H. REINHARD (1816-1892). Bei diesen Autoren kann fast ausnahmslos davon ausgegangen werden, dass das in der Liste aufgeführte Taxon (valid oder Synonym) auch aus Deutschland beschrieben wurde.

Die Nomenklatur und Systematik folgt den Vorstellungen von S. BELOKOBYLSKIJ. In einigen wenigen Fällen gibt es zwischen den Autoren unterschiedliche Auffassungen über die Artberechtigung einzelner Taxa. In entsprechenden Kommentaren werden diese erläutert. Die Gattungszuordnung wird von den Autoren oft unterschiedlich gehandhabt. Dort, wo es notwendig erschien (besonders im Hinblick auf eine ,nomenklatorische Kompatibilität” mit der demnächst erscheinenden „Fauna Europaea”), wurde in der Liste auch auf andere Gattungszuordnungen verwiesen.

In der Literatur gibt es häufig Widersprüche bzw. Unklarheiten bei der Datierung einiger Taxa. Darum wurde der Liste ein Abschnitt votangestellt, der sich mit den wesentlichsten Problemen auseinandersetzt (siehe: On the dates of several papers on Braconidae).

Soweit es möglich war, wurden die Arten aus der Liste ausgeschlossen (siehe: Species deleted from the German checklist), die irrtümlich (bzw. nur im historischen Kontext) für Deutschland gemeldet wurden. Ein derartiger Ausschluss ist oft problematisch, da Verbreitungsangaben nicht selten ohne Quelle kolportiert werden, wodurch unklar bleibt, ob es sich um einen eigenen Nachweis oder eine aus der Literatur übernommene Angabe handelt.

Für einige Arten müssen die Nachweise aus verschiedenen Gründen als unsicher angesehen werden (siehe: Records from Germany uncertain).

Es kann als sicher gelten, dass ein gewisser Anteil der Arten, die für Deutschland gemeldet wurden, auf Fehlbestimmungen basiert. Andererseits werden viele dieser Arten - unter Berücksichtigung ihrer Gesamtverbreitung - trotzdem in Deutschland vorkommen. Weiterhin sind zahlreiche Arten nur aus angrenzenden Staaten bekannt. Auch hier ist in der Regel mit einem Vorkommen in Deutschland zu rechnen.

In der vorliegenden Liste werden 1.485 Arten als nachgewiesen aufgeführt. Folgt man den Artauffassungen VAN ACHTERBERGs erhöht sich diese Zahl um etwa 10.

Aus Europa sind zur Zeit etwa 3.380 Braconiden-Arten bekannt (diese und folgende Zahlen nach VAN ACHTERBERG, in litt.). Obwohl die Niederlande (ca. 990 nachgewiesene Arten) und Österreich (ca. 760) über deutlich weniger nachgewiesene Arten verfügen, fehlen für bislang ca. 300 dieser Arten Nachweise aus Deutschland. Bei Betrachtung derartiger Zahlen ist es sicher nicht zu hoch gegriffen, wenn man für Deutschland mit etwa 2.000 Braconiden-Arten rechnet. 


\section{On the dates of several papers on Braconidae}

In the literature there are frequently contradictions and/or ambiguity regarding the dating of some taxa. Therefore the following chapers are given, which discuss the most important problems. The references were listed at the end of each chapter, in order to retain an overview.

The dates of publication of NEES VON ESENBECK's "Tchneumonides adsciti ..." (AT)

The dating of "Ichneumonides adsciti in genera et familias divisi" (NEES vON ESENBECK, $1811,1812,1816)$ often causes confusion. Especially the wrong year 1814 is applied often. According to SHERBORN (1922) the journal "Der Gesellschaft Naturforschender Freunde zu Berlin Magazin für die neuesten Entdeckungen in der gesamten Naturkunde. - Berlin (= Mag. Ges. Naturf. Freunde Berlin)" is to be dated as follows:I, 1807; II, 1808; III, 1809; IV, 1810; V (1-3),1811; V [1811](4),1812; VI (1-3), 1812; VI [1812](4), 1814; VII [1813](1-3), 1815; VII [1813](4), 1816; VIII [1814](1), 1816; VIII [1814](2), 1817; VIII [1814](3), 1817; VIII [1814](4), 1818.

The years in brackets [ refer to the intended year, the years without brackets refer to the year in which the journal really was published.

The correct references are as follows:

NEES VON ESENBECK, C. G. 1811: Ichneumonides adsciti in genera et familias divisi a Dre. Nees ab Esenbeck. - Der Gesellschaft Naturforschender Freunde zu Berlin Magazin für die neuesten Entdeckungen in der gesamten Naturkunde. Berlin 5(1): 3-37, Taf. I - II

NEES VON ESENBECK, C. G. 1812: Ichneumonides adsciti, in genera et familias divisi a Dre. Nees ab Esenbeck. Continuatio. - 1. c. 6(3): 183-222, Taf. IV

NEES VON ESENBECK, C. G. 1816: Ichneumonides adsciti, in genera et familias divisi. Ad Nees ab Esenbeck. Continuatio. - 1. c. 7[1813](4): 243-277, Taf. VII - VIII

Regarding NEES‘ later work (1834) see comments on HALIDAY's papers below.

References: SHERBORN, C. D. (1922): Introduction. Bibliogaphy.[a-anus]. - In: SHERBORN, C. D. (19221933): Index Animalium sive index nominum quae ab A. D. MDCCLVIII generibus et speciebus animalium imposita sunt. Sectio secunda a kalendis ianuariis, MDCCCI usque ad finem decembris, MDCCCL. - Cambridge, J. B. Peace Sect. II (1801-1850) (Part 2-33): cxxxvi u. 7056 u. vii u. cxxxiii-cxlvii u. 1098 p. Sect. II (1801-1850) (Part 2): cxxxvi p. u. 1-384

\section{PANZER's and HERRICH-SCHÄFFER's Braconidae in the „Faunae Insectorum Germanicae (AT)}

The correct references to PANZER are as follows:

PANZER, G. W. F. 1799: Faunae Insectorum Germanicae initia oder Deutschlands Insecten. - Nürnberg 6(72): 1-24 pl. (Ichneumon manducator at nr. 4). 
PANZER, G. W. F. 1804: Faunae Insectorum Germanicae initia oder Deutschlands Insecten. - Nürnberg 8[1805](92): 1-24 pl. (Bracon guttator at nr. 8)

According to SHERBORN (1923) the parts 85-96 of PANZER's „Faunae Insectorum Germanicae..." were published in 1804. Consequently, Bracon guttator PANZER was not described in 1805, but in 1804 .

In 1838 HERRICH-SCHÄFFER published several articles on braconids in the "Faunae Insectorum Germanicae initia oder Deutschlands Insecten". A number of references using the years 1835 or 1840 are wrong. The three important parts (Hefte) 153, 154, and 156 are published according to the wrappers on the 1st January, February and April 1838 respectively. The usually short articles are published on additional sheets without pagination, and the descriptions and figures are sometimes not in the same issue. Few species are described only by their figures (names on the wrapper), several species are described without figures. Though the title of the series "Faunae Insectorum Germanicae...") implies that all the species are described from Germany, some species are described from Austria (Sigalpbus longiseta, Eubadizon rufipes, Eubadizon laevis).

The references are as follows:

HERRICH-SCHÄFFER, G. A. W. (1838, January): Faunae Insectorum Germanicae initia oder Deutschlands Insecten. Regensburg 153: 1-24 + [4p.]: Uebersicht der Gattung Coelinius NEES. + [6p.]: Eubadizon, [5-6]: Uebersicht der Gattung Eubadizon NEES + [12p.]: Auseinandersetzung der Gattung Sigalphus LaTR.

HERRICH-SCHÄFFER, G. A. W. (1838, February): Faunae Insectorum Germanicae initia oder Deutschlands Insecten. Regensburg 154: 1-24 + [36p.]: Auseinandersetzung der Gattung Chelonus (JURINE).

HERRICH-SCHÄFFER, G. A. W. (1838, April): Faunae Insectorum Germanicae initia oder Deutschlands Insecten. Regensburg 156: 1-24 +[11p.]: Auseinandersetzung der Gattung Rogas NEES.

In the following overview are listed the references to HERRICH-SCHÄFFER's braconid species (alphabetical order).

153,154 and 156 are the numbers of the issue, unpaginated pages are given in brackets П, the other numbers refer to the sheets that are associated with the plates, or in the case of figures to the printed numbers on the plates. HERRICH-SCHÄFFER's numbers for the Chelonus species are given also in parentheses 0 .

Chelonus acuminatus: 154[4], [36] (nr. 32); Fig. 154.14.d.e.; bicarinatus: 154[4] (as "bicariantum", misspelling), [19] (nr. 18); Fig. 154.10.c.; bisulcatus. 154[2], [9] (nr. 5); Fig. 154.9.a.; emarginatus: 154[5], [36] (nr. 32); erosus: 154[5], [35] (nr. 31); Fig. 153.7; excisus: 154[2], [10] (nr. 6); Fig. 153.10; impressus: 154[2], [8] (nr. 4); Fig. 153.9; luteicornis: 154[4], [16] (nr. 15); Fig. 154.8; monilicornis: 154[4], [17] (nr. 16); Fig. 154.10.a.; productus: 154[5], [24] (nr. 21); Fig. 154.12.d; quadridens: 154[4], [18] (nr. 17); Fig. 154.10.b.; rimosus. Fig. 154.13.b; rufipes. 154[2], [11] (nr. 11); Fig. 154.9.b; similis: 154[3], [14] (nr. 13); Fig. 153.8; subemarginatus: 154[5], [36] (nr. 33); Fig. 154.6; subsulcatus: 154[4], [33] (nr. 28); Fig. 154.14.f.g; variabilis: 154[5], [23] (nr. 20); Coelinius bicarinatus: 153.18, 153[2]; depressus: 153[2], 153[5]; Fig. 156.15.d; flexuosus: 153[2], [4]; Fig. 156.13; gravis: 153[2], 153[6]; ruficollis: 153[2], 153[1], 154.22; Fig. 153.18, 154.22; 
Eubadizon aequator: 153[3], [6]; Fig. 153.20.b; angulator: Fig. 153.20.b; fuscipes: 153[2], [6]; laevis: 153[6], 153[4]; rufipes: 153[6], 153[3], 154.24; Fig. 153.20, 154.24.b;

Microgaster elegans: Fig. 153.14;

Perilitus brevicornis: 156[6]; Fig. 153.19; clavatus: 156[4]; distinguendus: 156[6]; erythrogaster: 156[5]; luteus: 156[7]; peregrinus: 156[8]; petiolaris: 156[6]; picipes: 156[7]; rufipes: 156[5]; ruralis: 156[8]; rutilus: 156[6];

Rogas affinis: 156[3]; albitibia: 156[8]; annulipes: 156[5]; Fig. 156.8; bifasciatus: 156[7]; compressor: 156[9]; dorsalis: 156[6]; Fig. 154.21; laevigatus: 156[8]; lanceolatus: 156[10]; longicaudis: 156[11]; miniatus: 156[6]; pallidicornis: 156[5]; pectoralis: 156[9]; picipes: 156[10]; pictus: 156[8]; ruficornis: 156[7]; Fig. 156.10; seriatus. 156[8]; Fig. 156.12; simplex: 156[9]; varius: 156[8]; Fig. 156.7;

Sigalphus affinis: 153.16, 153[2]; angustatus: 153[4], [10]; Fig. 153.22.b; brevicornis: 153[3], [7]; Fig. 153.21.b; foveolatus: 153[3], [5]; Fig. 153.21.a; fumatus: 153[4], [9]; Fig. 153.22.a; gracilis: 153.12, 153[3]; bilaris: 153.12, 153[3]; Fig. 153.12; longiseta: 153.16, 153[3]; pallidipennis. 153[4], [11]; Fig. 153.22.c, 153.24; mufipes. 153[4], [8]; Fig. 153.11; vitripennis. 153.16, 153[2]; Fig. 153.16.b.

Both WESMAEL and HERRICH-SCHÄFFER published in 1838 papers containing the same taxa, but HERRICH-SCHÄFFER has priority. WESMAEL published his paper after HERRICHSCHÄFFER because he cites in his "supplement" (p. 162-164) several times the papers by HERRICH-SCHÄFFER (CVA).

References: SHERBORN, C. D. (1923): On the Dates of G. W. F. PANZER's 'Fauna Insect. German.', 1792 1844. - The Annals and Magazine of Natural History, including Zoology, Botany, and Geology; Ninth Series, London 11(No. 64): 566-567

WESMAEL, C. 1838: Monographie des Braconides de Belgique. - Nouveaux Mémoires de l'Academie Royale des Sciences et Belles-Lettres de Bruxelles, Bruxelles 11: 167 p.

\section{The dates of the Braconidae described by CURTIS in the „British Entomology...” (A'T)}

BLACKWELDER (1947) published about the dates and the various editions of CUR'TIS „British Entomology..." and he pointed out that the first edition started on January 1,1824 and was published in 16 volumes of 12 parts each, or 770 plates in 192 parts. Each plate is dated and is accompanied by an additional text sheet with the same number as the plate. On the plate is figured one species (marked bold below), the other listed names are described or mentioned in the text only. The names (including the authors names) are listed as used by CURTIS. It should be noted, that CURTIS used several in litteris names of HALIDAY.

CURTTS, J. 1824-1840: British Entomology; being illustrations and descriptions of the genera of Insects found in Great Britain and Ireland: containing Coloured Figures from Nature of the most rare and beautiful species, and in many instances of the plants upon which they are found. - London, Author 16 vol. (each $12 \mathrm{nr}$ ): 2 p. text each per plate

[Vol. 2] pl. 69, May 1 1825: Bracon denigator Linné

[Vol. 2] pl. 73, June 1 1825: Bassus calculator Fabricius

[Vol. 3] pl. 141, Nov. 1 1826: Alysia Apii Nobis, apicalis Nob, similis Nob., pratellae Nob., gracilis Nob., pallida Nob., pubescens Nob., minuta Nob. 
[Vol. 6] pl. 289, Dec. 1 1829: Chaenon anceps Hal. MSS, anceps Curt. [sic], gracilis Hal., elegans Hal., viduus Hal., obscurus Curt., similis Curt., affinis Hal., fuliginosus Curt., cingulatus Hal., rufinotatus Curt., brevicornis $\mathrm{Hal}$., apterus Curt.

[Nol. 7] pl. 321, Aug. 1 1830: Microgaster alvearius Fab., annulipes Curt. (cocoon), vitripennis Curt., lacteipennis Curt., tibialis Curt., lineola Curt., gracilis Curt., atrator Curt., anomalon Curt.

[Vol. 8] pl. 383, Dec. 1 1831: Aphidius cirsii Curt., [A. pini Hal., infulatus Hal., pictus Curt., dimidiatus Curt., picipes Nees, fumatus Hal., basalis Curt. letifer Hal., minutus Curt., constrictus Hal. mentioned, but only in groups, not individually described, thus these names are not available]

[Vol. 9] pl. 415, Aug. 1 1832: Zele albiditarsus Curt., testaceator Curt., Z. ochraceator Curt. (n. nud.), Ephippium Curt., pectoralis Curt. (n. nud.), fulvifrons Curt. (n. nud.), thoracicus Curt., atrator Curt., longicauda Curt.

[Vol. 10] pl. 476, Nov. 1 1833: Leiophron apicalis Curt., mitis Haliday's MSS., Ochresiae Curt., pallipes Curt., picipes Curt., nitidus Curt., similis Curt., fulvipes Curt., pallidistigma Curt., basalis Curt.

[Vol. 11] pl. 507, July 1 1834: Hecabolus sulcatus Curt.

[Vol. 11] pl. 512, Aug. 1 1834: Rogas balteatus Hal. MSS, dispar Hal., ochraceus Curt., ater Curt., Subucola Curt., similis Curt., spathuliformis Curt., nobilis Hal.

[Vol. 14] pl. 672, Dec. 1 1837: Chelonus Wesmaelii Curt., Ascogaster Consobrinus Curt., pallidicornis Curt., fulviventris Curt., Esenbeckii Curt., Chelonus basalis Curt.

References: BLACKWELDER, R. E. 1947: The dates and editions of CURTIS'British Entomology. - Smithonian Miscellaneous Collections, Washington 107(5): 1-27

\section{The dates of publication of HALIDAY's papers on Braconidae (CvA \& AT)}

There exists some confusion concerning the exact dates of publication of HALIDAY's papers. For instance, HORN \& SCHENKLING (1928-1929) lists "1836", but FISCHER (1972) gives "1837" as the date of publication for the Opiinae part of HALIDAY's paper published in Ent. Mag. 4:203-221. This part is included in the third issue of the volume, which has on its frontispiece the date of completion of the whole volume, thus (at least) the last issue (together with the frontispiece) was published in 1837. Since HALIDAY's paper on the Opiinae is not in the last issue, it could be assumed (as was done by VAN ACHTERBERG, 1997) that HORN \& SCHENKLING (1928-1929) had good reasons for taking 1836 as date of publication. More confusing, HORN \& SCHENKLING date earlier papers of HALIDAY in the second issue of the joumal as 1837 (on Thysanoptera and Diptera, published in October 1836). However, on p. 197 of the volume 4 of the Ent. Mag. is given as date January 1837. Combining all the evidence (including the list of received journals published in the Mag. Nat. Hist. during 1835-1837), we artive at the dates for HALIDAY's publications on Braconidae as given in tab. 1.

When HALIDAY started his series of publications on Braconidae several other important publications on Braconidae appeared almost simultaneously, viz., CuRTIS, (1833, 1834, 1837), NEES (1834), BOUCHÉ (1834), WESMAEL (1835-1838), and HERRICH-SCHÄFFER (1838) describing partly the same species under different names (or same names in case of the CURTIS (1833, 1834)). HALIDAY's (1833a [April]) first publication on Braconidae pre- 
Tab 1: Dates of Haliday's publications on Braconidae

\begin{tabular}{|l|l|l|}
\hline Journal/book & pages & date \\
\hline Ent. Mag. 1(iii): & $259-276$ & $1833 \mathrm{a}$ (April) \\
\hline Ent. Mag. 1(v): & $480-491$ & $1833 \mathrm{~b}$ (October) \\
\hline Ent. Mag. 2(i): & $93-106$ & $1834 \mathrm{a}$ (January) \\
\hline Ent. Mag. 2(iii): & $225-259$ & $1834 \mathrm{~b}$ July) \\
\hline Ent. Mag. 2(v): & $458-468$ & $1835 \mathrm{a}$ (January) \\
\hline Ent. Mag. 3(i): & $20-45$ & $1835 \mathrm{~b}$ (April) \\
\hline Ent. Mag. 3(ii): & $121-147$ & $1835 \mathrm{c}$ (July) \\
\hline Ent. Mag. 4(i): & $38-59$ & $1836 \mathrm{a}$ (July) \\
\hline Ent. Mag. 4(ii): & $92-106$ & $1836 \mathrm{~b}$ (Oktober) \\
\hline Trans. Linn. Soc. London & $17: 316-331$ & $1836 \mathrm{c}$ \\
\hline Ent. Mag. 4(iii) & $203-221$ & 1837 (January) \\
\hline Ent. Mag. 5(iii): & $209-248$ & $1838 \mathrm{a}$ (July) \\
\hline Ent. Mag. 5(v): (addendum) & 519 & $1838 \mathrm{~b}$ (October) \\
\hline (Chapter in Westwood) & $61-65$ & $1839 \mathrm{a}$ (June) \\
\hline Hym. Brit. 2: & $1-28$ & $1839 \mathrm{a}$ (June) \\
\hline
\end{tabular}

dates CURTIS (1833b [November]), as is also evident because CURTIS cites HALIDAY's paper. HALIDAY (1834a January]) has priority over NEES (1834a [July or earlier, see WESMAEL, 1835]) and CURTIS, (1834 [August]); in part NEES (1834a-b) has priority over BOUCHE (1834 [December]), but only regarding the first volume (NEES, 1834a), as the second volume (NEES, 1834b) appeared after BOUCHÉ (1834), see NEES (1834b: 404). Ms P. GILBERT (former librarian of BMNH; in litt.) could not find an actual date of publication of the book by BoUCHÉ. Neither is further information available in the library of the DEI. In the absence of this knowledge it has to be assumed to have been available in December 1834; but the actual date is probably before December because NEES (1834b) refers to it. Finally, HALIDAY (1835a Danuary], 1835b [April], 1835c [July]) pre-dates WESMAEL (1835 [later than October, see HAESELBARTH, 1971]).

Considering the comments given above the following order of nomenclatural priority for the works published between 1833 and 1839 results:

CURTIS 1833 a (January): Ent. Mag. 1(ii): 186-199

HALIDAY 1833a (April): Ent. Mag. 1(iii): 259-276

HALIDAY 1833 b (October): Ent. Mag. 1(v): 480-491

CURTIS 1833 b (November 1): Brit. Ent. pl. 476

HALIDAY 1834a (January): Ent. Mag. 2(i): 93-106

NEES 1834 a (July or earlier): Hym. Ichn. aff. 1: 1-320

CURTIS 1834a (July): Brit. Ent. pl. 507

HALIDAY 1834b (July): Ent. Mag. 2(iii): 225-259

CurTIS 1834b (August 1): Brit. Ent. pl. 512 
BOUCHÉ 1834 (December or earlier, before NEES, 1834b): Naturgeschichte etc. 216 p. NEES 1834b (December or earlier, after BoUCHÉ, 1834): Hym. Ichn. aff. 2: 1-448

HALIDAY 1835a (January): Ent. Mag. 2(v): 458-468

HAIIDAY 1835b (April): Ent. Mag. 3(i): 20-45

HALIDAY 1835c (July): Ent. Mag. 3(ii): 121-147

WeSMAEL 1835 (later than October): Nouv. Mém. Acad. R. Sci. Bruxelles 9: 252 p. HALIDAY 1836a (July): Ent. Mag. 4(i): 38-59

HALIDAY 1836b (October): Ent. Mag. 4(ii): 92-106

HALIDAY 1836c: Trans. Linn. Soc. London 17: 316-331 (often listed as 1837, but on p. 573 of Mag. Zool. Bot. 1: dated 1836)

HALIDAY 1837 (January): Ent. Mag. 4(iii): 203-221

CURTIS 1837 (December 1): Brit. Ent. pl. 672

Wesmael 1837: Nouv. Mém. Acad. R. Sci. Bruxelles 10: 1+70 p.

HERRICH-SCHÄFFER 1838a-c (January, February, Aptil): Faun. Ins. Germ. 153, 154, 156 HALIDAY 1838a (April): Ent. Mag. 5(iii): 209-248

Wesmael 1838 (later than April): Nouv. Mém. Acad. R. Sci. Bruxelles 11: 167 p.

HALIDAY 1838b (October): Ent. Mag. 5(v): 519 (addendum)

HALIDAY 1839a (June): (chapter in WESTWOOD): 61-65 (according to Direction 32 ICZN)

HALIDAY 1839b: Hym. Brit. 2: 1-28

\section{References:}

ACHTERBERG, C. van 1997: Revision of the HALIDAY collection of Braconidae. - Zoologische Verhandelingen, Leiden 314: 1-115.

BOUCHÉ, P. F. 1834: Naturgeschichte det Garten Insecten. - Berlin: 216 p

CURTIS, J. 1833a: Characters of some undescribed Genera and Species, indicated in the "Guide to an Arrangement of British Insects". - The Entomological Magazine, London 1[1832-1833](2): 186-199.

CURTIS, J. 1833b: British Entomology; being illustrations and descriptions of the genera of Insects found in Great Britain and Ireland: containing Coloured Figures from Nature of the most rare and beautiful species, and in many instances of the plants upon which they are found. - London, Author [10] plate 476.

CURTIS, J. 1834a: dito - [11] plate 507.

CURTIS, J. 1834b: dito - [11] plate 512.

CURTIS, J. 1837: dito - [14] plate 672.

FISCHER, M. 1972: Hymenoptera, Braconidae (Opinae I). - Das Tierreich, Berlin 91: I-XII, 1-620.

HAESELBARTH, E. 1971: Notizen zur Gattung Pygostolus HALIDAY (Hymenoptera, Braconidae). - Opuscula Zoologica, München 112: 1-8.

HAIIDAY, A. H. 1833a: An Essay on the Classification of the Parasitic Hymenoptera of Britain, which correspond with the Ichneumones minuti of Linnaeus. - The Entomological Magazine, London 1[18321833](3): 259-276.

HALIDAY, A. H. 1833b: Essay on the Classification of Parasitic Hymenoptera, \&c. Of the Ichneumones of the Second Line, (Ichneumones adsciti, Essenbeck [sic!]). - The Entomological Magazine, London 1[1832-1833](5): 480-491.

HALIDAY, A. H. 1834a: dito - 2[1834-1835](1): 93-106.

HALIDAY, A. H. 1834b: dito - 2[1834-1835](3): 225-259. 
HALIDAY, A. H. 1835a: Essay on Parasitic Hymenoptera. Of the Ichneumones Adsciti. - The Entomological Magazine, Lotidon 2[1834-1835](5): 458-468.

HALIDAY, A. H. 1835b: dito - 3[1835-1836](1): 20-45.

HaLIDAY, A. H. 1835c: dito - 3[1835-1836](2): 121-147.

HALIDAY, A. H. 1836a: Essay on Parasitic Hymenoptera. - The Entomological Magazine, London 4[1836$1837](1): 38-59$.

HALIDAY, A. H. 1836b: dito - 4[1836-1837](2): 92-106.

HALIDAY, A. H. 1836c: Hymenoptera. - In: Curtis, J.; Haliday, A. H. \& Walker, F.: Descriptions of Insects collected by Capt. P. P. King in the Survey of the Straits of Magellan. Hymenoptera and Diptera. Trans. Linn. Soc. Lond. 17[1837]: 316-331.

HALidAY, A. H. 1837: Essay on Parasitic Hymenopteta. - The Entomological Magazine, London 4[18361837](3): $203-221$.

HALIDAY, A. H. 1838a: Essay on the Classification of Parasitic Hymenoptera. - The Entomological Magazine, London 5[1837-1838](3): 209-248.

HALIDAY, A. H. 1838b: Addenda to the genus Alysia. - The Entomological Magazine, London 5[1837$1838](5): 519$.

HALIDAY, A. H. 1839a: p. 61-65 In: WESTWOOD, J. O. [1839]: Synopsis of the Genera of British Insects. [1838-1840](E-F): 49-80. (Part of: WESTWOOD, J. O. 1838-1840: An Introduction to the modern Classification of Insects; founded on the natural habits and corresponding organisation of the different families. - London, Longman, Orme, Brown, Green, and Longmans 1; $2(16$ parts): [III]-XII-[XIII]+1462; [II]-XI+1-587+1-158(Synopsis)).

HALidAY, A. H. 1839b: Alysia. - Hymenoptera Britannica. - London, Ballière (Fasc. 2): 28 p.

HERRICH-SCHÄFFER, G. A. W. 1838a: Faunae Insectorum Germanicae initia oder Deutschlands Insecten. Regensburg 153: 1-24 + [4p.]: Uebersicht der Gattung Coelinius NEES. + [6p.]: Eubadizon, [5-6]: Uebersicht der Gattung Eubadizon NEES + [12p.]: Auseinandersetzung der Gattung Sigalpbus LATR.

HERRICH-SCHÄFFER, G. A. W. 1838b: dito - 154: 1-24 + [36p.]: Auseinandersetzung der Gattung Cbelonus JURINE)

HERRICH-SCHÄFFER, G. A. W. 1838c: dito - 156: 1-24 +[11p.]: Auseinandersetzung der Gattung Rogas NEES.

HORN, W. H. R. \& SCHENKLING, S. 1928-1929: Index Litteraturae Entomologicae, Serie I: die Welt-Literatur über die gesamte Entomologie bis inklusive 1863. - Berlin-Dahlem, Selbstverlag W. Horn 1-4: XXI p., $1426 \mathrm{p}$.

NEES VON ESENBECK, C. G. D. 1834a: Hymenopterorum Ichneumonibus affinium, Monographiae, genera Europaea et species illustratae. Volumen primum, Ichneumonidum Braconideorum Alysioideorum, tum Evanialium Monographias complectens. - Stuttgartiae et Tubingae, J. G. Cottae 1: XII+1-320.

NEES VON ESENBECK, C. G. D. 1834b: Hymenopterorum Ichneumonibus affinium, Monographiae, genera Europaea et species illustratac. Volumen secundeum, Pteromalinorum, Codrinorum et Dryineorum Monographias complectens. - Stuttgartiae et Tubingae, J. G. Cottae 2: 1-448.

Wesmael, C. 1835: Monographie des Braconides de Belgique. - Nouveaux Mémoires de l'Academie Royale des Sciences et Belles-Lettres de Bruxelles, Bruxelles 9:252 p.

WESMAEL, C. 1837: dito - 10: 1+70 p.

WESMAEL, C. 1838: dito - 11: 167 p.

\section{The dates of C. G. THOMSON's works on Braconidae (AT)}

SHENEFELT (1965) gave a list of THOMSON's works on Braconidae, all published in THOMSON's Opuscula Entomologica. He attributed the works published in vol. 17 to the year "[1892]1894". Later he used several different years $(1891,1892,1894)$ for the species in the volumes 16 and 17 without a comprehensible system (SHENEFELT 19691978). The wrappers of both volumes are dated "MDCCCXCII". There is no evidence, 
that a year other than 1892 is to be assumed for these volumes. The vol. 17 is mentioned later (1894) in the Zoological Record, but with reference to the year 1892. Therefore THOMSON's papers seem to have been published as follows:

THomson, C. G. 1874: Öfversigt af Sveriges Sigalpher. - Opuscula Entomologica edidit C. G. THOMSON, Lund 6: 553-588

-1892: XLIV. Bidrag till Braconidernas kännedom. - 1. c. 16: 1659-1751

-1892: Bidrag till Braconidernas kännedom. - 1. c. 17: 1777-1861

-1892: XLVIII. Bidrag till Sveriges insectfauna. - 1. c. 17: 1862

-1895: LII. Bidrag till Braconidernas kännedom. - 1. c. 20: 2141-2339

-1897: LVII. Bidrag till Sveriges insectfauna. - 1. c. 22: 2451-2452

\section{References:}

SHENEFELT, R. D. 1965: A contribution towards knowledge of the world literature regatding Braconidae (Hymenoptera: Braconidae), - Beiträge zur Entomologie, Berlin 15(3-4): 243-500.

SHENEFELT, R. D. 1969-1978: Braconidae 1-10. - Hymenopterorum Catalogus (nova editio) 4-7, 9-13, 15 : 1-1872. - 's-Gravenhage (10: SHENEFELT, R. D. \& MARSH, P. M.; Index - Braconidae 11: 16 ACHTERBERG, C. VAN \& SHENEFELT, R. D., 1980, 384 pp.).

\section{The dates of publication of MARSHALL's volumes in 'Species des Hyménoptères d'Europe \& d'Algérie" on Braconidae (CvA)}

Dates according to the Zoological Record (but for 1893 after the "Archiv für Naturgeschichte").

MARSHALt, T. A. 1888-1900: Les Braconides. - Species des Hyménoptères d'Europe \& d'Algérie. - Beaune (Côte-d'Or)

Tome IV: fasc. 28-32: pp. 1-324, pls i-v. 1888

fasc. 33-35: pp. 325-492, pls vii-x. $\quad 1889$

fasc. $36+37$ : pp. 493-609, pls xi-xviii. $\quad 1890$

Tome V: fasc. 38: pp. 1-136, pls 1-2 ～(? Febr.) 1891

pp. 137-240, pls 1893

pp. 241-336, pls 1894a

pp. 337-400, pls ?1894b

pp. 401-480, pls 12-14 1895

pp. $481-635$, pls $15-20 \quad$ (May) 1896

Tome Vbis: fasc. 39: pp. i-viii + 1-144, pls 1-6 1897

pp. 145-288, pls 7-12 1898

pp. 289-334, pls 13-15 1899

pp. 337-373, pls \& 1-24 (? + 25-82) 1900 


\section{A note on GRIFFITHS' papers on Alysiinae (AT)}

G. C. D. GRIFFITHS published in the Beiträge zur Entomologie, Berlin between 1964 and 1984 seven papers on Alysiinae. According to the files of the DEI the part II was distributed after January 25, 1967. Therefore, it is very likely that also part III was published in 1967 . The references are as follows:

GrIfFITHS, G. C. D. 1964-1984: The Alysiinae (Hym. Braconidae) parasites of Agromyzidae (Diptera).

- 1964: I. General questions of taxonomy, biology and evolution. - Beiträge zur Entomologie, Berlin 14(7-8): 823-914.

- 1967a: II. The parasites of Agromyza FALLÉN. - Beiträge zur Entomologie, Berlin 16[1966](5-6): 551-605

- 1967b: III. The parasites of Parapbytomyza ENDERLEIN, Pbytagromyza HENDEL and Pbytomyza FALLÉN. - Beiträge zur Entomologie, Berlin 16[1966](7-8): 775-951

- 1967c: IV. The parasites of Hexomyza ENDERLEIN, Melanagromyza HeNDEL, Opbiomyia BRASCHNIKOV and Napomyza WESTWOOD. - Beiträge zur Entomologie, Berlin 17(58): 653-696.

- 1968a: V. The parasites of Liriomyza M匹K and certain small genera of Phytomyzinae. Beiträge zur Entomologie, Berlin 18(1-2): 5-62.

- 1968b: VI. The parasites of Cerodontha RONDANI s. 1. - Beiträge zur Entomologie, Berlin 18(1-2): 63-152.

- 1984: VII. Supplement. - Beiträge zur Entomologie, Berlin 34(2): 343-362.

\section{List of species recorded from Germany}

The list includes - apart from the species names that are considered to be valid - in brackets [] a source (a museum or reference from literature, the latter marked with \#). Furthermore the synonyms are listed in parentheses. During the final preparation of the checklist three taxa were deleted from the list. Thus the highest registration number (1488) does not agree with number of species recorded for Germany.

Other Abbreviations:
AT - A. TAEGER
CVA - C. VAN ACHTERBERG
EH - E. HAESELBARTH
SB - S. A. BELOKOBYLSKIJ

CVA provided hitherto unpublished information about synonymies and valid names. This is mainly based on unpublished revisional work on Opiinae, the Leiophron complex, Eubazus, Syntretus and Aleiodes, all of the West Palaearctic. This is to avoid problems in future, especially to achieve more compatibility with the system that will be applied in the "Fauna Europaea" (VAN ACHTERBERG in prep.). More than 30 synonyms are mentioned as "unpublished synonyms" and are not to be considered as "syn. nov." CvA will explain the relevant changes and synonymies in a later paper. 


\section{Adeliinae}

1 Adelius clandestinus (FÖRSTER, 1851) [\# 86]

2 Adelius determinatus (FÖRSTER, 1851) [ZMHUB] (= concinnus Ruthe)

3 Adelius dubius (FÖRSTER, 1851) [ZMHUB]

4 Adelius erytbronotus (FÖRSTER, 1851) [ZMHUB] (= flavus Tobias; pyrrhia Beirne)

$5 \quad$ Adelius germanus (HALIDAY, 1834) [\# 86]

6 Adelins byalimipennis (FÖRSTER, 1851) [\# 86] (= hyalipennis Förster misspell.)

7 Adelius parnulus (FÖRSTER, 1851) [\# 86]

8 Adelius subfasciatus HALIDAY, 1833 [ZMHUB] (= minutissimus Zetterstedt)

$9 \quad$ Adelizus viator (FÖRSTER, 1851) [\# 86]

\section{Agathidinae}

10 Agathis anglica MARSHALl, 1885 [\# 87] (= albanica Fischer; caucasica Tobias; longicauda Kokujev; marshalli Fahringer; syriaca Fischer; taiwanensis Chou \& Sharkey)

11 Agathis assimilis KOKUJEV, 1895 [ZSM] (= anchisiades Nixon; propinqua Kokujev)

12 Agathis breviseta NEES, 1812 [\# 86]

13 Agatbis fuscipennis (ZETTERSTEDT, 1838) [ZSM] (= albicostellac Fischer; artemesiana Fischer; glabricula Thomson; meridionellae Fischer; rostrata sensu Nixon; schmiedeknechti Kokujev)

14 Agathis griseifrons THOMSON, 1895 [ZSM] (= laticarpa Telenga)

15 Agatbis lugubris (FÖRSTER, 1862) [ZSM] (= minuta Niezabitowski)

16 Agathis malvacearum LATREILLE, 1805 [ZSM] (- panzeri Jurine)

17 Agathis nigra NEES, 1812 [ZSM] (= kasachstanica Tobias; nixoni Belokobylskij \& Jervis; testaceipes Fischer)

18 Agatbis rufipalpis NEES, 1812 [\# 60]

19 Agathis semiaciculata IvANov, 1899 [\#32] (= striolata Shestakov)

20 Agathis syngenesiae NEES, 1812 [\# 86] (= gilvus Papp; insularis Snellen van Vollenhoven; syngenesii Nees misspell.; tadzhica Telenga)

21 Agathis tibialis NEES, 1812 [\# 87] (= genualis Marshall)

22 Agathis umbellatarum NEES, 1812 [\# 86] (= aurantiaca Fahringer; brullaei Lucas; gussakovskyi Tobias; kolazyi Fischer; thoracica Lucas)

23 Agathis varipes THOMSON, 1895 [\# 86] (= ariadne Nixon; dissimilis Shestakov; glabricollis Telenga; lederi Fischer; rufipes Ivanov; serratulae Tobias; simulatrix Kokujev)

24 Bassus arcuatus (REINHARD, 1867) [\# 88]

25 Bassus brevicaudis (REINHARD, 1867) [\# 88]

26 Bassus calculator (FABRICIUS, 1798) [ZSM] (= abscissus Ratzeburg)

27 Bassus cingulipes (NEES, 1812) [\# 88]

28 Bassus claustbalianus (RATZEBURG, 1844) [\# 88]

29 Bassus conspicuus (WESMAEL, 1837) [ZSM] (= zonatus Marshall)

30 Bassus dimidiator (NEES, 1834) [\# 88] (= angulator Ratzeburg misspell.; cingulator Ratzeburg)

31 Bassus fortipes (REINHARD, 1867) [\# 88]

32 Bassus linguarius (NEES, 1812) [\# 88] (= kaszabi Papp; minor Enderlein; minorend Shenefelt)

33 Bassus mediator (NEES, 1812) [\# 88] (= lugubrator Ratzeburg) 
34 Bassus nugax (REINHARD, 1867) [\# 88] (= rufiventris Abdinbekova)

35 Bassus pumilus (RATZEBURG, 1844) [\# 88]

36 Bassus rufipes (NEES, 1812) [\# 88] (= germanica Enderlein)

37 Bassus rugulosus (NEES, 1834) [\# 88] (= punctatus Abdinbekova)

38 Bassus tegularis (THOMSON, 1895) [\# 88]

39 Bassus tumidulus (NEES, 1812) [\# 88] (= annae Enderlein; annuligaster Fulmek; anuphrievi Tobias; intermedius Ivanov; rufa Fahringer; ruficoxis Fahringer; victoris Telenga)

40 Bassus raykovi (NIXON, 1986) [ZSM]

41 Cremnops desertor (LINNE, 1758) [ZSM] (= alternans Enderlein; atricornis Smith; deflagrator Nees; deflagrator Spinola; emniscatus Enderlein)

42 Disophrys caesa (KLUG, 1835) [\# 86] (= anthracina Kriechbaumer; caesia Klug misspell.; imperialis A. Costa; inculcator auct.; inculcator Linné misident.; initiator Fonscolombe)

43 Earinus elator (FABRICIUS, 1804) [ZSM] (= major Fonscolombe; nitidulus Nees; pilosus Tobias; thoracicus Nees)

44 Earinus gloriatorius (PANZER, 1809) [\# 60] (= affinis Wesmael; bicingulatus Thomson; delusor Wesmael; gloratorius Panzer misspell.; gloriator Nees; niger Zetterstedt; ochropes Lyle; ruficoxis Fahringer; tuberculatus Wesmael)

Rbampbagathis nasicomis (TELENGA, 1955) [\# 60]

\section{Alysiinae}

46 Adelurola florimela (HALIDAY, 1838) [ZSM] (= multiarticulata Marshall; pentapleuroides Fischer) Remark: According to QUICKE et al. (1997 [\# 96]) the species belongs to the genus Dapsilarthra (based on internal morphology) (CvA).

47 Alloea bonessi FISCHER, 1966 [\# 86]

48 Alloea contracta HALIDAY, 1833 [DET]

49 Alloea lonchopterae FISCHER, 1966 [\# 90]

50 Alysia atra HALIDAY, 1838 [ZSSM]

51 Absia cingulata NEES, 1834 [\# 86]

52 Alysia frigida HALIDAY, 1838 [DE]

53 Absia fuscipennis HALIDAY, 1838 [\# 92] (= obscuripes Thomson)

54 Alysia incongria NEES, 1834 [\# 86]

55 Alysia lucia HALDAY, 1838 [ZSM] (= diversiceps Fischer; rudis Tobias)

56 Alysia lucicola HALIDAY, 1838 [\#91]

57 Alysia mandibulator (NEES, 1812) [\# 92] (= loripes Haliday)

58 Aly'sia manducator (PANZER, 1799) [ZSM] (= apicalis Curtis; bucephala Marshall; curtungula Thomson; similis Curtis; stercoraria Latreille)

59 Alysia rufidens NEES, 1834 [DEI]

60 Alysia similis (NEES, 1812) [\# 86]

61 Alysia sophia HALIDAY, 1838 [\# 86]

62 Alysia tipulae (SCOPOLI, 1763) [ZMHUB] (= abdominator Nees; curtangula Thomson; notabilis Förster)

63 Alysia triangulator (NEES, 1812) [\# 86]

64 Alysia truncator (NEES, 1812) [\# 86]

65 Alysia umbrata STELFOX, 1941 [\#92] 
66

68

69

70

71

72

73

74

75

76

77

78

79

80

81

82

83

84

85

86

87

88

89

90

91

92

93

94

95

96

97

98

99

100

101

102

103

Amyras clandestina (HALIDAY, 1839) [\# 54]

Anisocyrtaperdita (HALIDAY, 1838) [ZMHUB]

Antrusa flawicoxa. (THOMSON, 1895) [\# 27] Remark: According to QUICKE et al. (1997 [\# 96]) the species fits better in Dacnusa (CvA).

Antrusa melanocera (THOMSON, 1895) [ZSM] (= persimilis Nixon) Remark: Probably the species belongs to Exotela ( $\mathrm{CVA})$.

Antrusa vaenia (NIXON, 1954) [\# 54] Remark: Probably the species belongs to Exotela (CvA).

Apbaereta cepbalotes (HALIDAY, 1833) [\# 86]

Apbaereta difficilis NIXON, 1939 [ZSM]

Aphaereta falcigera GRAHAM, 1960 [\# 41]

Aphaereta major (THOMSON, 1895) [ZSM]

Apbaereta minuta (NEES, 1811) [ZSM] (= fuscipes Nees)

Apbaereta scaptomyzae FISCHER, 1966 [ZSM] (= minutissima Tobias)

Aphaereta tenuicornis NIXON, 1939 [\# 86]

Apronopa baeselbarthi VAN ACHTERBERG, 1980 [ZSM]

Aristelix pboenicura (HALIDAY, 1839) [ZSM] (= phaenicura Haliday misspell.)

Asobara mufescens (FÖRSTER, 1862) [\# 86]

Asobara tabida (NEES, 1834) [ZSM]

Aspilota abrburgensis FISCHER, 1974 [\# 17]

Aspilota efoveolata (THOMSON, 1895) [\# 86] (= pneumatica Fischer)

Aspilota fuscicomis (HALIDAY, 1838) [\# 86] (= minutus Nees)

Aspilota birticornis (THOMSON, 1895) [\# 86]

Aspilota microcera (THOMSON, 1895) [\# 86]

Aspilota nervulata FISCHER, 1974 [\# 17]

Aspilota ruficornis (NEES, 1834) [\# 05]

Asyntactus rbogaleus MARSHALL, 1898 [ZMHUB]

Asyntactus sigalphoides MARSHALL, 1898 [\# 13]

Atopandrium debilitatum (MORLEY, 1933) [ZSM] (= conflucta Fischer; loripenne Graham)

Remark: According to WHARTON it belongs to Aphaereta (CvA).

Cbaenusa conjungens (NEES, 1811) [\# 41] (= conjugens Nees misspell.)

Chaenusa naiadum (HALIDAY, 1839) [\# 86]

Cbaenusa natator (SCHULZ, 1907) [\# 86]

Chaenusa nereidum (HALIDAY, 1839) [\# 40]

Chasmodon apterus (NEES, 1812) [ZSM]

Chorebus abrota (NIXON, 1945) [\# 50]

Chorebus agraules (NIXON, 1945) [\# 27]

Chorebus albimarginis GRIFFITHS, 1967 [\# 24]

Cborebus albipes (HALIDAY, 1839) [\# 24]

Chorebus alecto (MORLEY, 1924) [STUTTG] (= turissa Nixon)

Chorebus amauromyzae GRIFFITHS, 1968 [\# 26]

Chorebus ampliator (NEES, 1834) [ZMHUB] 
104

105

106

107

108

109

110

111

112

113

114

115

116

117

118

119

120

121

122

123

124

125

126

127

128

129

130

131

132

133

134

135

136

137

138

139

140

141

142

143

144

145

Chorebus aphantus (MARSHALL, 1895) [STUT'TG]

Chorebus armida NIXON, 1945 [STUTTG]

Chorebus artemisiellus GRIFFITHS, 1968 [\# 26]

Chorebus asramenes (NIXON, 1943) [\# 27]

Chorebus atis (NIXON, 1943) [\# 47]

Cborebus anesta (NIXON, 1944) [STUT'TG]

Cborebus batbyzonus (MARSHALL, 1895) [ZSM]

Chorebus bensoni (NIXON, 1943) [\# 28]

Chorebus brevicomis (THOMSON, 1895) [\# 25] (= chrysippe Nixon; ea Nixon)

Cborebus bubri GRIFFITHS, 1967 [\#24]

Cborebus cbenopodii GRIFFITHS, 1984 [\# 28]

Chorebus cinctus (HALIDAY, 1839) [\# 48]

Chorebus claripennis GRIFFITHS, 1984 [\#28]

Chorebus coxator (THOMSON, 1895) [\# 40]

Cborebus credne (NIXON, 1944) [STUTTG]

Cborebus crenulatus (THOMSON, 1895) [\# 50] (= elegantula Nixon)

Cborebus cubocepbalus (TELENGA, 1934) [\# 51] (= cyclops Nixon)

Chorebus gylindricus (TELENGA, 1934) [\# 25] (= cybele Nixon)

Cborebus cyparissa (NIXON, 1944) [ZSM]

Chorebus yytherea (NIXON, 1937) [STUTTG] (= calliope Nixon; tesmia Nixon)

Cborebus dagda (NIXON, 1943) [\#24]

Cborebus daimenes (NIXON, 1945) [\# 26]

Chorebus deione (NIXON, 1944) [STUTTG]

Chorebus difficilis GRIFFITHS, 1968 [STUTTG]

Cborebus diremtus (NEES, 1834) [\# 51]

Chorebus dirona (NrXON, 1945) [\#26]

Chorebus endymion GRIFFITHS, 1967 [\# 24]

Chorebus enephes (NIXON, 1945) [\# 27]

Chorebus ergias (NIXON, 1945) [\# 24]

Chorebus esbelta (NIXON, 1937) [\# 53]

Chorebus eucodonis GRIFFITHS, 1984 [\# 28]

Chorebus fallax (NIXON, 1937) [\# 24]

Chorebus flavipes (GOUREAU, 1851) [STUT'TG] (= raissa Nixon)

Chorebus fordi (NIXON, 1954) [\# 86]

Chorebus foveolus (HALIDAY, 1839) [\# 40]

Chorebus fuscipennis (NIXON, 1937) [ZSM]

Chorebus galii GRIFFITHS, 1984 [\# 28]

Chorebus ganesa (NIXON, 1945) [\# 27]

Cborebus gedanensis (RATZEBURG, 1852) [\# 86] (= anguligena Nixon)

Chorebus gentianellus GRIFFITHS, 1967 [STUTTG]

Chorebus glaber (NIXON, 1944) [\# 49]

Chorebus glabriculus (THOMSON, 1895) [\#37] 
146 Chorebus gnapbalii GRIFFITHS, 1967 [\# 24]

147 Chorebus groscbkei GRIFFITHS, 1967 [STUTTG]

148 Chorebus beringianus GRIFEITHS, 1967 [\# 25]

149 Chorebus interstitialis (THOMSON, 1895) [ZSM] (= mamertes Nixon) Remark: According to GRIFFITHS (1964 [\#22]) in Exotela but seems to fit in Trichocborebus TOBIAS (CvA).

150 Chorebuskama (NIXON, 1945) [\# 41]

151 Cborebus knautiae GRIFFITHS, 1967 [\# 28]

152 Chorebus lateralis (HALIDAY, 1839) [STUTTG] (= fuscula Haliday)

153 Chorebus leptogaster (HALIDAY, 1839) [ZSM] (= naenia Morley)

154 Cborebus limnicola (NEES, 1812) [\# 86]

155 Chorebus limoniadum (MARSHALL, 1896) [\# 40]

156 Chorebus lonchopterae (RUSCHKA, 1926) [\# 86]

157 Chorebus longicomis (NEES, 1811) [ZMHUB] (= affinis Nees)

158 Chorebus lugubris (NTXON, 1937) [STUTTG]

159 Chorebus lurvlae GRIFFITHS, 1967 [\# 24]

160 Chorebus melanopbytobiae GRIFFITHS, 1968 [\# 26]

161 Chorebus merellus (NIXON, 1937) [STUTTG]

162 Chorebus misellus (MARSHALL, 1895) [STUTTG]

163 Chorebus mucronatus (TELENGA, 1934) [\# 86]

164 Chorebus myles (NIXON, 1943) [\# 47]

165 Chorebus nana (NIXON, 1943) [STUTTG]

166 Chorebus navicularis (NEES, 1812) [\# 86]

167 Chorebus nerissa (NIXON, 1937) [ZSM]

168 Chorebus ninella (NIXON, 1945) [\# 27]

169 Cborebus nobilis GRIFEITHS, 1968 [STUTTG]

170 Chorebus nomia (NIXON, 1937) [ZSM]

171 Chorebus nydia (NIXON, 1937) [\# 37]

172 Cborebus obscuripes (RUSCHKA, 1913) [\# 86]

173 Cborebus oritias (NIXON, 1945) [\# 28]

174 Cborebus ovalis (MARSHALL, 1896) [\# 50]

175 Chorebus parvungula (THOMSON, 1895) [\# 25] (= acco Nixon)

176 Chorebus pelion (NIXON, 1944) [STUTTG]

177 Cborebus perkinsi (NIXON, 1944) [\#23]

178 Chorebus petiolatus (NEES, 1834) [ZSM]

179 Chorebus phaedra (NIXON, 1937) [ZSM]

180 Chorebus poemyzae GRIFFITHS, 1968 [\# 27]

181 Chorebus polygoni GRIFFITHS, 1967 [\# 23]

182 Chorebus pospelovi (KURDJUMOV, 1912) [\# 86]

183 Chorebus posticus (HALIDAY, 1839) [\# 86] (= dentatus Tobias; egregia Marshall; gracilis Nees)

184 Chorebus pseudomisellus GRIFFITHS, 1968 [STUTTG]

185 Chorebus pulchellus GRIFFITHS, 1967 [\# 25]

186 Chorebus punctus (GOUREAU, 1851) [STUTTG] 
187 Cborebus resa (NIXON, 1937) [\# 86]

188 Chorebus rbanis (NIXON, 1943) [ZSM]

189 Cborebus rondanii (GIARD, 1904) [ZSM] (= galba Nixon)

190 Chorebus rostratae GRIFFITHS, 1984 [\# 28]

191 Chorebus mbicundus GRIFFITHS, 1968 [\# 27]

192 Chorebus rufipes (NEES, 1812) [\# 86]

193 Chorebus scabiosae GRIFFITHS, 1967 [STUTTG]

194 Chorebus senilis (NEES, 1812) [ZSM]

195 Chorebus sera (NIXON, 1937) [\# 49]

196 Chorebus solstitialis (STELFOX, 1952) [\# 23]

197 Chorebus stagnalis (HEYMONS, 1908) [\# 86]

198 Chorebus stenocentrus (THOMSON, 1895) [\# 28]

199 Chorebus stilifer GRIFFITHS, 1968 [STUTTG]

200 Chorebus subfuscus GRIFFITHS, 1968 [\# 26]

201 Chorebus sylvestris GRIFFITHS, 1967 [STUTTG]

202 Chorebus talaris (HALIDAY, 1839) [ZSM]

203 Chorebus tanis (NIXON, 1945) [STUTTG]

204 Chorebus thecla (NIXON, 1943) [\#24]

205 Chorebus thisbe (NixON, 1937) [\#23]

206 Cborebus thusa (NIXON, 1937) [\# 41]

207 Chorebus transversus (NIXON, 1954) [STUTTG]

208 Chorebus trilobomyzae GRIFFITHS, 1968 [STUTTG]

209 Cborebus uliginosus (HALIDAY, 1839) [\# 86] (= thienemanni Ruschka)

210 Cborebus uma (NIXON, 1944) [STUTTG]

211 Cborebus venustus (TOBIAS, 1962) [\# 26]

212 Chorebus veratri GRIFFITHS, 1968 [STUTTG]

213 Chorebus xipbidius GRIFFITHS, 1967 [ZSM]

214 Cborebus xylostellus GRIFFITHS, 1967 [STUTTG]

215 Coelinidea depressa (HERRICH-SCHÄFFER, 1838) [\# 86]

216 Coelinidea elegans (CURTIS, 1829) [ZSM] (= brevicornis Curtis; cingulatus Curtis; rufinotatus Curtis; similis Curtis)

217 Coelinidea gracilis (CURTIS, 1829) [ZSM]

218 Coelinidea gravis (HERRICH-SCHAFFER, 1838) [\# 86]

219 Coelinidea nigra (NEES, 1811) [\# 86] (= niger Nees)

220 Coelinidea ruficollis (HERRICH-SCHÄFFER, 1838) [\# 86] (= procera Haliday)

221 Coelinidea stenostigma (THOMSON, 1895) [ZSM]

222 Coelinidea vidua (CURTIS, 1829) [\# 86] (= ater Curtis)

223 Coelinius parvulus (NEES, 1811) [ZSM] (= anceps Curtis; anceps Schiödte; bicarinatus HerrichSchäffer; bicolor Marechal; circulator Gravenhorst; flexuosus Herrich-Schäffer; rimator Schiödte) Remark: Ichneumon circulator GRAVENHORST, 1807 is a primary homonym of Ichneumon circulator PANZER, 1801. Thus the combination "Coelinius circulator" cannot be applied for the species ( $\mathrm{CVA}$ ).

224 Colonewra dice (NIXON, 1943) [STUTTG] 
225 Coloneura stylata FÖRSTER, 1862 [ZMHUB] (= exilis Förster; oligomera Förster; stilata Förster misspell.; tates Nixon)

226 Cratospila circe (HALIDAY, 1838) [ZMHUB]

227 Dacnusa abdita (HALIDAY, 1839) [\# 23] (= incidens Thomson)

228 Dacnusa adducta (HALIDAY, 1839) [\# 54] Remark: Belongs to the subgenus Agonia (SB), that can be considered to be a valid genus ( $\mathrm{CvA}$ ).

229 Dacnusa alpestris GRIFFITHS, 1967 [\# 24]

230 Dacnusa angelicina GRIFFITHS, 1967 [\# 24]

231 Dacnusa aquilegiae MARSHALL, 1896 [STUTTG]

232 Dacnusa areolaris (NEES, 1811) [\# 24]

233 Dacnusa austriaca (FISCHER, 1961) [\# 26]

234 Dacnusa brevistigma (TOBIAS, 1962) [\# 24]

235 Dacnusa centaureae GRIFFITHS, 1967 [STUTTG]

236 Dacnusa cerpheres (NIXON, 1948) [\# 28] (= cepheres Nixon misspell.)

237 Dacnusa cingulator (NEES, 1834) [\# 86]

238 Dacnusa confinis RUTHE, 1859 [ZSM]

239 Dacnusa delpbinii GRIFFITHS, 1967 [\# 24]

240 Dacnusa discolor (FÖRSTER, 1862) [\# 26]

241 Dacnusa faeroeensis (ROMAN, 1917) [ZSM] (= lestes Nixon)

242 Dacnusa gentianae GRIFFITHS, 1967 [STUTTG]

243 Dacnusa groschkeana GRIFFITHS, 1968 [STUTTG]

244 Dacnusa beringi GRIFFITHS, 1967 [\# 24]

245 Dacnusa bospita (FÖRSTER, 1862) [STUTTG] Remark: Belongs to the subgenus Aphantia (SB), that can be considered to be a valid genus $(\mathrm{CvA})$.

246 Dacnusa laeta (NIXON, 1954) [\# 54]

247 Dacnusa laevipectus THOMSON, 1895 [STUTTG]

248 Dacnusa liopleuris THOMsON, 1895 [\# 26]

249 Dacnusa lissos (NIXON, 1954) [STUTTTG]

250 Dacnusa lithospermi GRIFFITHS, 1967 [STUTTG]

251 Dacnusa longiradialis NIXON, 1937 [ZSM]

252 Dacnusa lonicerella GRIFFITHS, 1967 [\# 24]

253 Dacnusa lugens (HALIDAY, 1839) [\# 52]

254 Dacnusa macrospila (HALIDAY, 1839) [ZSM] (= monospila Haliday misspell.)

255 Dacnusa maculipes THOMSON, 1895 [ZSM]

256 Dacnusa maxima (FISCHER, 1961) [\# 23]

257 Dacnusa melicerta (NIXON, 1954) [\#24]

258 Dacnusa merope (NIXON, 1948) [\# 24]

259 Dacnusa ogroe NixON, 1937 [STUTTG] (= ocyrae Nixon misspell.)

260 Dacnusa plantaginis GRIFFITHS, 1967 [STUTTG]

261 Dacnusa pubescens (CURTIS, 1826) [ZSM] (= exserens Nees)

262 Dacnusa sasakawai TAKADA, 1977 [\# 28] Remark: Belongs to the subgenus Aphantia (SB), that can be considered to be a valid genus $(\mathrm{CVA})$.

263 Dacnusa sibirica TELENGA, 1934 [\# 26] (= comes Nixon) 
264 Dacnusa soldanellae GRIFFITHS, 1967 [STUTTG]

265 Dacnusa stramineipes (HALIDAY, 1839) [\# 28]

266 Dacnusa tarsalis THOMSON, 1895 [STUTTG] (= nitetis Nixon)

267 Dacnusa temula (HALIDAY, 1839) [\# 54]

268 Dapsilarthra apii (CURTIS, 1826) [\# 86]

269 Dapsilarthra balteata (THOMSON, 1895) [ZSM] Remark: Belongs to the subgenus Heterolexis (SB), that can be considered according to QUICKE et al. (1997 [\# 96]) to be a valid genus (CvA).

270 Dapsilartbra dictynna (MARSHALL, 1895) [ZSM] Remark: Belongs to the subgenus Heterolexis (SB), that can be considered according to QUICKE et al. (1997 [\# 96]) to be a valid genus (CVA).

271 Dapsilartbra isabella (HALIDAY, 1838) [DET Remark: Belongs to the subgenus Grammospila $(\mathrm{SB})$, that can be considered to be a valid genus ( $\mathrm{CVA}$, in prep.).

272 Dapsilarthra rufiventris (NEES, 1812) [ZSM] (= flaviventris Haliday) Remark: Belongs to the subgenus Grammospila (SB), that can be considered to be a valid genus (CvA, in prep.).

273 Dapsilartbra subtilis (FÖRSTER, 1862) [ZMHUB] (= testacea Griffiths) Remark: Belongs to the subgenus Heterolexis (SB), that can be considered according to QUICKE et al. (1997 [\# 96]) to be a valid genus (CvA).

274 Dapsilarthra sylvia (HALIDAY, 1839) [\# 26] (= carpathica van Achterberg)

275 Dinotrema amplisignatum (FISCHER, 1973 [\# 18] (= spiniphorae Fischer) Remark: Aspilota spiniphorae FISCHER, 1985 is an unpublished synonym of amplisignatum (CvA).

276 Dinotrema brevicorne (NEES, 1812) [\# 86]

277 Dinotrema concinnum (HALIDAY, 1838) [\# 41] (= maxima Fischer)

278 Dinotrema dimidiatum (THOMSON, 1895) [\# 86]

279 Dinotrema erythropa FÖRSTER, 1862 [ZMHUB]

280 Dinotrema falsificum STELFOX \& GRAHAM, 1950 [\# 86] (= falcificum Stelfox \& Graham misspell.)

281 Dinotrema latifemur (FISCHER, 1974) [\# 17]

282 Dinotrema macrocera (THOMSON, 1895) [\# 86]

283 Dinotrema nervosum (HALIDAY, 1833) [\# 86] (= crassicosta 'Thomson)

284 Dinotrema perlustrandum (FISCHER, 1973) [\# 16]

285 Dinotrema pullum (FÖRSTER, 1862) [\# 86]

286 Dinotrema pusillum (NEES, 1812) [\# 86]

287 Dinotrema reductidens (FISCHER, 1974) [\# 17]

288 Dinotrema speculum (HALIDAY, 1838) [ZSM] (= venusta Haliday)

289 Epimicta marginalis (HALIDAY, 1839) [\# 40]

290 Eudinostigma latistigma (FISCHER, 1962) [\# 19]

291 Exotela aconiti GRIFFITHS, 1967 [ZSM]

292 Exotela arunci GRIFFITHS, 1967 [STUTTG]

293 Exotela chromatomyiae GRIFFITHS, 1984 [\# 28]

294 Exotela yyclogaster FÖRSTER, 1862 [ZSM] (= bellina Nixon)

295 Exotela bera (NIXON, 1937) [ZSM]

296 Exotela latbyri GRIFFITHS, 1984 [\# 28] 
297 Exotela minuscula GRIFFITHS, 1967 [\# 24] (= miniscula Griffiths misspell.)

298 Exotela nowakowskï GRIFFITHS, 1967 [ZSM]

299 Exotela obscura GRIFFITHS, 1967 [ZSM]

300 Exotela pbryne (NIXON, 1954) [\#30]

301 Exotela senecionis GRIFFITHS, 1967 [STUTTG]

302 Exotela soncbina GRIFFITHS, 1967 [STUTTG]

303 Exotela spinifer (NIXON, 1954) [ZSM]

304 Exotela umbellina (NIXON, 1954) [ZSM]

305 Idiasta dichrocera KÖNIGSMANN, 1960 [ZMHUB]

306 Idiasta maritima (HALIDAY, 1838) [\# 40]

307 Idiasta nepbele (HALIDAY, 1838) [\# 40$]$

308 Idiasta paramaritima KÖNIGSMANN, 1960 [DED]

309 Idiastapicticomis (RUTHE, 1854) [\# 86]

310 ldiasta westphalica KÖNIGSMANN, 1960 [\# 42]

311 Laotris striatula (HALDAX, 1839) [STUTTG]

312 Leptotrema dentifemur (STELFOX, 1943) [\# 86]

313 Mesocrina indagatrix FöRSTER, 1862 [ZSM] (= venatrix Marshall)

314 Ortbostigma cratospilum (THOMSON, 1895) [\# 43]

315 Ortbostigma laticeps (THOMSON, 1895) [ZSM]

316 Orthostigma longicome KÖNIGSMANN, 1969 [ZSM]

317 Orthostigma maculipes (HALIDAY, 1838) [\# 40]

318 Orthostigma mandibulare (TOBIAS, 1962) [ZSM]

319 Ortbostigma pseudolaticeps KÖNIGSMANN, 1969 [\# 43]

320 Ortbostigma pumila (NEES, 1834) [ZSM]

321 Ortbostigma sculpturatum TOBIAS, 1962 [ZSM]

322 Orthostigma sordipes (THOMSON, 1895) [ZSM]

323 Panerema inops FörSTER, 1862 [ZMHUB]

324 Pentapleura angustula (HALIDAY, 1838) [ZMHUB] (= laevipleuris Tobias)

325 Pentapleura fuliginosa (HALIDAY, 1838) [\# 86] (= carinata Thomson)

326 Pentapleura pumilio (NEES, 1812) [\# 86] (= mesocrinoides Goidanich)

327 Pentapleura tenuicomis (FÖRSTER, 1862) [ZMHUB] Remark: In the past treated as a synonym of angustula, but it represents a valid species (CvA, in prep.).

328 Phaenocarpa bicolor (FÖRSTER, 1862) [ZMHUB] (= bicolorina Shenefelt)

329 Phaenocarpa canaliculata STELFOX, 1941 [ZSM]

330 Phaenocarpa carinthiaca FISCHER, 1970 [ZSM]

331 Pbaenocarpa conspurcator (HALIDAY, 1838) [ZSM] (= arctica Thomson; tatrica Niezabitowski)

332 Phaenocarpa engenia (HALIDAY, 1838) [ZSM] (= piceator Zetterstedt)

333 Phaenocarpa flavipes (HALIDAY, 1838) [ZSM]

334 Pbaenocarpagalatea (HALIDAY, 1838) [\# 40]

335 Phaenocarpa lichasberstovi'TELENGA, 1935 [ZSM]

336 Phaenocarpa livida (HALIDAY, 1838) [ZSM] (= debilis Förster)

337 Phaenocarpamaria (HALIDAY, 1838) [\# 40] 
Phaenocarpa nitida (THOMSON, 1895) [\# 40]

339 Phaenocarpapicinervis (HALIDAY, 1838) [ZSM]

340 Phaenocarpa pratellae (CURTIS, 1826) [\# 86]

341 Phaenocarpapsalliotae TELENGA, 1935 [DED]

342 Pbaenocarpapullata (HALIDAY, 1838) [ZSM]

344 Phaenocarpa ruficeps (NEES, 1812) [ZSM] (= ferga Papp; testaceus Nees)

345 Phaenocarpa theodori (SNELLEN VAN VOLLENHOVEN, 1878) [DE]]

346 Pbaenocarpa ungularis (THOMSON, 1895) [ZSM]

347 Polemochartus aboletus PAPP, 1992 [RMNH]

348 Polemocbartus liparae (GIRAUD, 1863) [ZSM]

349 Polemochartus melas (GIRAUD, 1863) [ZSM]

350 Protodacnusa aridula (THOMSON, 1895) [\# 22] (= miser Nixon)

351 Protodacnusa ruthei GRIFFITHS, 1964 [\# 22]

352 Protodacnusa tristis (NEES, 1834) [ZSM] (= longistigma Telenga)

353 Sarops rea NIXON, 1942 [ZSM]

354 Symphanes aciculata FÖRSTER, 1862 [ZMHUB]

355 Synaldis concolor (NEES, 1812) [\#19] Remark: The species belongs according to CVA to Dinotrema.

356 Synaldis distrata (NEES, 1834) [\# 19] Remark: The species belongs according to CvA to Dinotrema.

357 Synaldis parvicornis (THOMSON, 1895) [\# 19] Remark: The species belongs according to CvA to Aspilota.

358 Synorasis fucicola (HALIDAY, 1838) [\# 37]

359 Syncrasis halidayi (FÖRSTER, 1862) [\# 37] (= fuscipes Haliday)

360 Synelix semirugosa (HALIDAY, 1839) [ZMHUB] (= amaurosomae Telenga)

361 Tanycarpa amplipennis (FÖRSTER, 1862) [ZMHUB]

362 Tanycarpa bicolor (NEES, 1812) [\# 86] (= ancilla Haliday)

363 Tanycarpa gracilicornis (NEES, 1812) [\# 86]

364 Tanycarpa rufinotata (HALIDAY, 1838) [ZMHUB] (= carinata Förster; erythrogaster Förster; foersteri Shenefelt)

365 Tates heterocera ('THOMSON, 1895) [\# 86]

366 Tracbionus bians (NEES, 1816) [ZMHUB]

367 Trachionus mandibularis (NEES, 1816) [ZMHUB]

368 Tracbionus ringens (HALIDAY, 1839) [ZMHUB]

369 Tracbyusa aurora (HALIDAY, 1838) [ZSM]

\section{Aphidiinae}

370 Aclitus obscunipennis FÖRSTER, 1862 [ZMHUB]

371 Adialytus ambigutus (HALIDAY, 1834) [\# 46] (= arvicola Starý; crocinus Mackauer; mackaueri Stary)

372 Adialytus salicaphis (FITCH, 1855) [\# 46] (= laticephalus Telenga; obsoletus sensu Ratzeburg)

373 Adialytus tenuis FÖRSTER, 1862 [ZMHUB]

374 Adialytus thelaxis (STARÝ, 1961) [\# 46] 
375 Apbidius aquilus MACKAUER, 1961 [\# 46] (= aquilis Mackauer misspell.; sicarius Mackauer)

376 Apbidius asteris HALIDAY, 1834 [\# 46] (= absinthii Marshall; absintii Marshall misspell.; lutescens Haliday)

377 Apbidius avenae HALIDAY, 1834 [\# 46] (= caraganae Starý; crithmi Marshall; granarius Marshall; hungaricus Györfi; pascuorum Marshall; picipes Nees)

378 Apbidius cingulatus RUTHE, 1859 [\# 45] (= gregarius Marshall; pterocommae Ashmead; pterocommae Marshall)

379 Apbidius colemani VIERECK, 1912 [\# 10] (- transcaspicus Telenga)

380 Apbidius eglanteriae HALIDAY, 1834 [\# 46]

381 Aphidius ervi HALIDAY, 1834 [ZSM] (= fumipennis Györfi; infirmus Nees; mirotarsi Starý; ulmi Marshall)

382 Aphidius funebris MACKAUER, 1961 [\# 46] (= cirsii Ivanov ?; eriophori Mackauer)

383 Apbidius bieracionm STARÝ, 1962 [ZSM]

384 Apbidius bortensis MARSHALL, 1896 [\# 46] (= berberidis Smith)

385 Apbidius matricariae HALIDAY, 1834 [\# 46] (= affinis Quilis; arundinis Haliday; baudysi Quilis; cirsii Haliday; dyscritus Quilis; goidanichi Quilis; lychnidis Marshall; merceti Quilis; nigriteleus Smith; obscuriforme Quilis; phorodontis Ashmead; polygoni Marshall; renominatus Hincks; valentinus Quilis)

386 Aphidius phalangomyzi STARÝ, 1963 [ZSM]

387 Apbidius rhopalosiphi DE STEFANI, 1902 [\# 10] (= equiseticola Starý; indivisus Quilis; poacearum Starý)

388 Apbidius ribis HALIDAY, 1834 [\# 46]

389 Apbidius rosae HALIDAY, 1834 [\# 46] (= protaeus Wesmael; rosarum Nees)

390 Apbidius salicis HALIDAY, 1834 [\# 46] (= dauci Marshall; donci Marshall; restrictus Nees)

391 Apbidius schimitscheki (STARÝ, 1960) [\# 46]

392 Apbidius setiger (MACKAUER, 1961) [\# 46]

393 Aphidius sonchi MARSHALL, 1896 [\# 46]

394 Apbidius tanacetarius MACKAUER, 1962 [\# 46] (= tanaceti Curtis nom. nud.; tanaceticola Starý)

395 Apbidius urticae HALIDAY, 1834 [\# 46] (= aulacorthi Starý; longulus Marshall; nigrescens Mackauer; rubi Starý; silvaticus Starý)

396 Apbidius wabekistanicus LUZHETZKI, 1960 [\# 46] (= impressus Mackauer)

397 Areopraon lepelleyi (WATERSTON, 1926) [\# 46]

398 Areopraon pilosum MACKAUER, 1959 [ZMHUB]

399 Calapbidius elegans MACKAUER, 1961 [\# 46]

400 Diaeretellus epbippizm (HALIDAY, 1834) [DEI]

401 Diaeretellus beinzei (MACKAUER, 1959) [\# 46] (= heinzi Mackauer misspell.)

402 Diaeretellus macrocarpus MACKAUER, 1961 [\# 46]

403 Diaeretellus palustris STARÝ, 1971. [\# 90]

404 Diaeretiella rapae (M'INTOSH, 1855) [\# 46] (= aphidum Mukerii \& Chatterjee; brassicae Marshall; californicus Baker; chenopodiaphidis Ashmead; chenopodii Förster; crawfordi Rohwer; croaticus Quilis; napus Quilis; nipponensis Viereck; obsoletus Kurdjumov; piceus Cresson; plesiorapae Blanchard; vulgaris Bouché ?) Remark: If the synonymy is correct, $D$. vulgaris (BOUCHÉ, 1834) would be the ralid name for the species (CvA).

405 Diaeretus leucopterus (HALIDAY, 1834) [\# 46] 
406

407

408

409

410

411

412

413

414

415

416

417

418

419

420

421

422

423

424

425

426

427

428

429

430

431

432

433

434

435

436

437

438

439

Dyscritulus planiceps (MARSHALL, 1896) [\# 46]

Ephedrus cerasicola STARÝ, 1962 [\# 46]

Ephedrus chaitophori GÄRDENFORS, 1986 [\#21]

Ephedrus lacertosus (HALIDAY, 1833) [\# 45] (= homostigma Fahringer; muesebecki Smith)

Ephedrus laevicollis (THOMSON, 1895) [\#21] (= brevicomis Nees; minor Stelfox)

Ephedrus nacheri (QUILIS, 1934) [\# 46]

Ephedrus niger GAUTIER, BONNAMOUR \& GAUMONT, 1929 [\# 46] (= campestris Starý)

Ephedrus persicae FROGGATT, 1904 [\# 45] (= holmani Starý; impressus Granger; interstitialis Watanabe; nevadensis Baker; nitidus Gahan; palaestinensis Mackauer; pulchellus Stelfox; vidalis Quilis)

Ephedrus plagiator (NEES, 1811) [ZMHUB] (= japonicus Ashmead; parcicornis Nees)

Ephedrus validus (HALIDAY, 1833) [\# 45]

Falciconus pseudoplatani (MARSHALL, 1896) [\# 46]

Lipolexis gracilis FÖRSTER, 1862 [ZMHUB] (= palpator Gautier \& Bonnamour; pieltaini Quilis)

Lysapbidus arvensis STARÝ, 1960 [\# 46]

Lysaphidus viaticus SEDLAG, 1969 [\# 90]

Lysiphlebus confusus TREMBLAY \& EADY, 1978 [\# 45] (= ambiguus auct.)

Lysipblebus dissolutus (NEES, 1811) [ZMHUB] (= macrocornis Mackauer)

Lysipblebus fabarum (MARSHALL, 1896) [\# 45] (= aphidiperda Rondani ?; aurantii Pierantoni; cardui Marshall; gomezi Quilis; inermis Quilis; innovatus Quilis; janinii Quilis; monilicomis Thomson; moroderi Quilis) Remark: If the synonymy is correct, $L$. aphidiperda (RONDANI, 1874) would be the valid name of the species (CvA).

Lysipblebus fritamuelleri MACKAUER, 1960 [\# 46]

Lysipblebus birticornis MACKAUER, 1960 [\# 46]

Metaphidius aterrimus (FAHRINGER, 1935) [\# 46] (= trioxiformis Stary \& Sedlag)

Misapbidus acalephae (MARSHALL, 1896) [\# 46] (= rietscheli Mackauer; urticae Mackauer)

Remark: The species of Misaphidius usually have been treated before in the genus Trioxys.

The reasons for the new combinations will be given in the Fauna Europaea (CvA).

Misaphidus angelicae (HALIDAY, 1833) [ZSM] (= boscai Quilis; fumariae Quilis; granatensis Quilis; mediterraneus Mackauer; obscuriformis Quilis; placidus Gautier; wollastonii Cabrera)

Misapbidus brevicornis (HALIDAY, 1833) [ZMHUB]

Misapbidus centaureae (HALIDAY, 1833) [ZMHUB]

Misapbidus genistae (MACKAUER, 1960) [\# 46]

Misapbidus beraclei (HALIDAY, 1833) [ZMHUB] (= obsoletus Wesmael; variegator Szépligeti)

Misaphidus letifer (HALIDAY, 1833) [\# 46]

Monoctonus caricis (HALIDAY, 1833) [\# 46]

Monoctonus cerasi (MARSHALL, 1896) [\# 46]

Monoctonus crepidis (HALIDAY, 1834) [\# 46]

Monoctonus ligustri VAN ACHTERBERG, 1989 [\# 06]

Monoctonus nervosus (HALIDAY, 1833) [\# 46] (= biroi Györfi; breviantennatum Starý)

Paralipsis enervis (NEES, 1834) [\# 46]

Pauesia abietis (MARSHALL, 1896) [\# 46]

DOI: $10.21248 /$ contrib.entomol.53.2.341-435 
440 Pauesia cupressobii (STARÝ, 1960) [\# 46]

441 Pawesia infulata (HALIDAY, 1834) [\# 46] (= albiflagellaris Starý)

442 Pawesiajuniperorum (STARÝ, 1960) [\#46]

443 Panesia laricis (HALIDAY, 1834) [\# 46]

444 Pauesia piceacollis (STARÝ, 1960) [\# 46]

445 Pawesia picta (HALIDAY, 1834) [\# 46]

446 Pawesiapini (HALIDAY, 1834) [\# 46] (=varius Nees)

447 Panesia pinicollis (STARÝ, 1960) [\# 46]

448 Pawesia siluestris (STARÝ, 1960) [\# 46]

449 Pauesia spatulata SEDLAG \& STARÝ, 1980 [\# 90]

450 Pauesia unilachni (GAHAN, 1927) [\# 45] (= basilewskyi Benoit; praevisus Gautier \& Bonnamour)

451 Praon absintbii BIGNELL, 1894 [\# 46]

452 Praon barbatum MACKAUER, 1967 [\# 46]

453 Praon bicolor MACKAUER, 1959 [\# 46]

454 Praon caricicola MACKAUER, 1967 [\# 46]

455 Praon dorsale (HALIDAY, 1833) [ZMHUB] (= grossum Starý; longicorne Marshall)

456 Praon exsoletum (NEES, 1811) [ZMHUB] (= exoletum Nees misspell.; palitans Muesebeck)

457 Praon flawinode (HALIDAY, 1833) [ZMHUB] (= flavonode Haliday misspell.)

458 Praon gallicum STARÝ, 1971 [\# 10]

459 Praon lemantinum GAUTIER, 1922 [\# 46]

460 Praon myzophagum MACKAUER, 1959 [\# 46]

461 Praon necans MACKAUER, 1959 [\# 46]

462 Praon spinosum MACKAUER, 1959 [\# 46]

463 Praon volucre HALIDAY, 1833 [\# 45] (= angulator Nees; aphidivorus Ratzeburg; pruni Ivanov)

464 Protaphidius wissmannii (RATZEBURG, 1848) [ZMHUB] (= formicaria Goidanich)

465 Toxares deltiger (HALIDAY, 1833) [\# 45] (= flaveolus Györfi)

466 Trioxys auctus (HALIDAY, 1833) [ZMHUB]

467 Triaxys betulae MARSHALL, 1896 [\# 46]

468 Trioxys cirsii (CURTIS, 1831) [\# 46] (= aceris Haliday)

469 Trioxys complanatus QuILIS, 1931 [\# 46] (= utilis Muesebeck)

470 Trioxys compressicomis RUTHE, 1859 [\# 46] (= testaceus Stelfox)

471 Trioxys cumicandus MACKAUER, 1967 [\# 46]

472 Trioxys falcatus MACKAUER, 1959 [\# 46]

473 Trioxys bortorum STARY, 1960 [\# 46] (= affinis Mackauer)

474 Trioxys bumuli MACKAUER, 1960 [\# 46]

475 Trioxys izipbyae MACKAUER, 1967 [\# 46]

476 Trioxys lambersi MACKAUER, 1960 [\# 46]

477 Trioxys pallidus (HALIDAY, 1833) [\# 46]

478 Trioxys pannonicus STARÝ, 1960 [\# 46]

479 Trioxys parauctus STARÝ, 1960 [\# 46] 


\section{Brachistinae}

480 Eubazus abieticola VAN ACHTERBERG \& KENIS, 2000 [\# 01]

481 Eubazus aequator (HERRICH-SCHÄFER, 1838) [\# 86] (= angulator Herrich-Schäffer) Remark: The names refer to the same figure in the original description. Consequently, both names are objective synonyms. Eubadizon angulator HERRICH-SCHÄFFER, 1838 is considered as syn. nov. of Eubadizon aequator HERRICH-SCHÄFFER, 1838 (AT).

482 Eubazus angustimus (RUTHE, 1867) [\# 86] (= angustinus Ruthe misspell.)

483 Eubaqus carinatus (NEES, 1816) [\# 86]

484 Eubaマus claviventris (RUTHE, 1867) [DET]

485 Eubazus corrugatus (RUTHE, 1867) [ZSM]

486 Eubarus cruentatus (RUTHE, 1867) [\# 86]

487 Eubarus destitutus (RATZEBURG, 1848) [\# 86]

488 Eubazus exsertor (REINHARD, 1867) [ZMHUB]

489 Eubaqus fasciatus (NEES, 1816) [DET] (= fuscipalpis Wesmael)

490 Eubazus flavipes (HALIDAY, 1835) [\# 86] (= laevis Herrich-Schäffer)

491 Eubarus fuscipes (HERRICH-SCHÄFFER, 1838) [ZMHUB] (= nigripes Ruthe)

492 Eubarus gallicus (REINHARD, 1867) [ZMHUB]

493 Eubazus interstitialis (RATZEBURG, 1844) [\# 86]

494 Eubazus lepidus (HALIDAY, 1835) [\# 86]

495 Eubazus longicaudis (RATZEBURG, 1844) [\# 86] (= denticulatus van Achterberg)

496 Eubarus macrocephalus NEES, 1812 [\# 86] (= ratzeburgi Fischer; synchitae Hedquist; xiphydriae Tobias) Remark: Eubadizon ratreburgi FISCHER, 1962 and Eubazus xipbydriae TOBIAS, 1986 are hitherto unpublished synonyms (CvA).

497 Eubarus mimutus (RATZEBURG, 1848) [ZSM]

498 Eubazus nigricoxis (WESMAEL, 1835) [ZSM]

499 Eubazus pallipes NEES, 1812 [ZMHUB] (= coxalis Nees; pallidipes Marshall; pallipede Rondani; pollipes Kirchner; semistriatus Haliday)

500 Eubaqus parvulus (RUTHE, 1867) [ZMHUB]

501 Eubazus punctatus (RATZEBURG, 1852) [\# 86]

502 Eubazus robustus (RATZEBURG, 1844) [\# 86] (= firmus Ratzeburg; infirmus Leonardi; noctuae Ratzeburg; strigator Thomson; tenthredinum Hartig)

503 Eubazus mficoxis (WESMAEL, 1835) [\# 86] (= ruficornis Kirchner; politus Ratzeburg) Remark: Eubazus politus RATZEBURG, 1852 is a hitherto unpublished synonym (CvA).

504 Eubazus rugosus (RATZEBURG, 1848) [\# 86]

505 Eubazus semirugosus (NEES, 1816) [DEI] (= arete Fahringer; atricornis Ratzeburg; curculionum Hartig; glabrata Fahringer; mucronatus Thomson; rufipes Herrich-Schäffer; truncatus Thomson; tuberculator Zetterstedt)

506 Eubazus sigalpboides (MARSHALL, 1889) [\# 40]

507 Eubazus tibialis (HALIDAY, 1835) [ZSM] (= uncigenis Wesmael)

508 Eubarus vagus (RUTHE, 1867) [\# 86]

509 Eubazus vitripennis (HERRICH-SCHÄFFER, 1838) [\# 86]

510 Foersteria puber (HALIDAX, 1835) [DE] (= opacus Reinhard; talitzkii 'Tobias)

511 Polydegmon foveolatus (HERRICH-SCHÄFFER, 1838) [\# 86] (= intermedius Szépligeti)

512 Polydegmon sinuatus FörSTER, 1862 [ZMHUB] 
513 Schizoprymms actaphractus SNOFLAK, 1953 [ZSM]

514 Schizoprymnus ambiguus (NEES, 1816) [\# 86]

515 Scbizoprymnus angustatus (HERRICH-SCHÄFFER, 1838) [\# 86]

516 Schizoprymns crassiceps (THOMSON, 1892) [\# 86] (= ambiguus Thomson)

517 Schizoprymnus fumatus (HERRICH-SCHÄFFER, 1838) [\# 86]

518 Schizoprymnus hilaris (HERRICH-SCHÄFFER, 1838) [\# 80] (= ambiguus Wesmael; antennalis Thomson; obscurus Thomson)

519 Schizoprymnus longiseta (HERRICH-SCHÄFFER, 1838) [\# 86]

520 Schizoprymnus obscurus (NEES, 1816) [\# 86]

521 Schizoprymnus opacus (THOMSON, 1892) [DED]

522 Schiroprymnus pallidipennis (HERRICH-SCHÄFFER, 1838) [\# 86] (= strigosa Fahringer)

523 Schizoprymnus parvus (THOMSON, 1892) [\# 86]

524 Schizoprymnus pullatus (DAHLBOM, 1833) [\# 86] (= bimaculatus Fahringer; globosus Szépligeti; rufipes Herrich-Schäffer)

525 Triaspis affinis (HERRICH-SCHÄFFER, 1838) [\# 86] Remark: The generic name Triaspis $=$ "three (parted) shield") is feminine and not masculine as "aspis" is feminine (CvA).

526 Triaspis brevicornis (HERRICH-SCHÄFFER, 1838) [\# 86] Remark: The species seems to fit better in Schizoprymnus (CvA).

527 Triaspis breviventris (THOMSON, 1892) [ZSM]

528 Triaspis caudata (NEES, 1816) [\# 86] (= arcticus Hellén; australis Szépligeti; gracilis Herrich-Schäffer)

529 Triaspis collaris (THOMSON, 1874) [\# 40]

530 Triaspis complanellae (HARTIG, 1847) [ZSM] (= companellae Hartig misspell.; flavipes Ivanov)

531 Triaspis facialis (RATZEBURG, 1852) [\# 86]

532 Triaspis flawipalpis (WESMAEL, 1835) [\# 86]

533 Triaspis floricola (WESMAEL, 1835) [ZSM]

534 Triaspis luteipes (THOMSON, 1874) [\# 86]

535 Triaspis obscurella (NEES, 1816) [ZSM] (= aciculatus Ratzeburg; similis Szépligeti) Remark: Sigalphus aciculatus RATZEBURG, 1848 is a hitherto unpublished synonym of obscurella (CVA).

536 Triaspispallipes (NEES, 1816) [\# 86] (= fulvipes Haliday)

537 Triaspis podlussanyi PAPP, 1998 [\# 80]

538 Triaspis rimulosa (THOMSON, 1892) [\# 40]

539 Triaspis striatula (NEES, 1816) [\# 86]

540 Triaspis striola (THOMsON, 1874) [\# 86]

\section{Braconinae}

541 Atanyolus denigrator (LINNÉ, 1758) [DET] (= albiscutis Telenga)

542 Atanycolus fulviceps (KRIECHBAUMER, 1898) [DEI]

543 Atanycolus genalis (THOMSON, 1892) [DET (= initiator auct.)

544 Atanycolus neesii (MARsHaLL, 1897) [DED]

545 Atanycolus petiolaris (THOMSON, 1892) [\# 86]

546 Atanycolus sculpturatus (THOMSON, 1892) [DE] (= ivanovi Kokujev; ivanowi Kokujev misspell.; signatus Szépligeti) 
547 Baryproctus barypus (MARSHALL, 1885) [\# 86] (= caucasicus Telenga; hungaricus Szépligeti)

548 Bracon abbreviator NEES, 1834 [DEI] (= fraudator Marshall)

549 Bracon albipennis NEES, 1834 [\# 86]

550 Bracon apricus SCHMIEDEKNECHT, 1896 [\# 86]

551 Bracon arcuatus THOMSON, 1892 [\# 86]

552 Bracon atrator NEES, 1834 [ZMHUB]

553 Bracon atrorufus NEES, 1834 [\# 86]

554 Bracon balteatus MARSHALL, 1897 [\# 86]

555 Bracon bicellularis RATZEBURG, 1852 [\# 86]

556 Bracon bipartitus WESMAEL, 1838 [DED] (= otiosus Marshall)

557 Bracon bracbycerus THOMSON, $1892[$ [ 86] (= kudsiricus Papp)

558 Bracon brevicornis WESMAEL, 1838 [\# 86]

559 Bracon breviusculus WESMAEL, 1838 [\# 86]

560 Bracon brumescens FAHRINGER, 1928 [\# 86]

561 Bracon caudatus RATZEBURG, 1848 [ZMHUB]

562 Bracon caudiger NEES, 1834 [\# 86]

563 Bracon claripennis THOMSON, 1892 [ZSM]

564 Bracon colpophorus WESMAEL, 1838 [ZSM]

565 Bracon coniferarum FAHRINGER, 1928 [\# 86]

566 Bracon conjugellae BENGTSSON, 1924 [DED]

567 Bracon crassiceps THOMSON, 1892 [ZSM]

568 Bracon delibator HALIDAY, 1833 [DED] (= anthracinus Nees)

569 Bracon delusor SPINOLA, 1808 [\# 86]

570 Bracon dicbromus WESMAEL, 1838 [\# 61]

571 Bracon discoideus WESMAEL, 1838 [ZMHUB]

572 Bracon epitriptus MARSHALL, 1885 [ZSM] (= epitriphus Marshall misspell.; maculifer Szépligeti; novus Szépligeti) Remark: If $B$. nowus is considered to be valid: also recorded from Germany (ZSM; det. Papp).

573 Bracon erraticus WESMAEL, 1838 [DED] ( $=$ confinis Szépligeti; erythrostictus Marshall; foveola Thomson; hades Papp; lagodechianus Telenga; planiceps Telenga; superciliosus Wesmael; talitzkii Telenga; transcaspicus Telenga)

574 Bracon exarator MARSHALL, 1885 [\# 86]

575 Bracon exbilarator NEES, 1834 [ZSM] (= satanas Wesmael; striolatus Thomson; tibialis Zetterstedt)

576 Bracon fasciatorior SHENEFELT, 1978 [\# 86] (= fasciator Spinola)

577 Bracon flagellaris THOMSON, 1892 [\# 63] (= dallatorrei Szépligeti; facialis Thomson; quinquemaculatus auct.; thomsonii Dalla Torre)

578 Bracon flavipes NEES, 1834 [\# 86]

579 Bracon fortipes WESMAEL, 1838 [\# 86]

580 Bracon fulvipes NEES, 1834 [ZSM] (= kiritshenkoi Telenga)

581 Bracon fuscicoxis WESMAEL, 1838 [\# 86]

582 Bracon fuscipennis WESMAEL, 1838 [\# 86]

583 Bracon grandiceps THOMSON, 1892 [DEI] (= crassiusculus Szépligeti; gallicus Thomson) 
584 Bracon guttator PANZER, 1804 [\# 86]

585 Bracon guttiger WESMAEL, 1838 [DEI]

586 Bracon bebetor SAY, 1836 [DEI] (= breviantennatus De Stefani; dorsator Say; juglandis Ashmead)

587 Bracon bylobii RATZEBURG, 1848 [DEI]

588 Bracon immutator NEES, 1834 [DET] (= efoveolatus Thomson)

589 Bracon instabilis MARSHALL, 1897 [\# 83]

590 Bracon intercessor NEES, 1834 [DEI] (= adjectus Szépligeti; bisinuatus Szépligeti; fallaciosus Szépligeti; fulvus Szépligeti; fumigatus Szépligeti; hemirugosus Szépligeti; kacheticus Telenga; maslovskii Telenga; mixtus Szépligeti; nitidiusculus Szépligeti; rhynchiti Grese; rufiscapus Szépligeti; segregatus Telenga; suspectus Szépligeti; vigilax Kokujev) Remark: If $B$. fulvus is considered to be valid: also recorded from Germany (ZSM; det. Papp).

591 Bracon labrator RATZEBURG, 1844 [\# 86]

592 Bracon laetus WESMAEL, 1838 [\# 80]

593 Bracon laevigatissimus DALLA TORRE, 1898 [\# 86] (= laevigatus Ratzeburg; scutellaris Ratzeburg)

594 Bracon larvicida WESMAEL, 1838 [\# 64]

595 Bracon leptus MARSHALL, 1897 [DED] (= centaureae Szépligeti; obscuricornis Szépligeti; rufipalpis Szépligeti; rufipedator Szépligeti)

596 Bracon livida TELENGA, 1936 [\# 82]

597 Bracon longicollis WESMAEL, 1838 [DE] (= alutaceus Szépligeti; aluteceus Szépligeti misspell.; crassicauda Thomson; depressiusculus Szépligeti; rugulosus Szépligeti; subcylindricus Wesmael) Remark: If $B$. rugolosus is considered to be valid: also recorded from Germany (ZSM; det. Papp).

598 Bracon longulus THOMSON, 1892 [ZSM]

599 Bracon mariae DALLA TORRE, 1898 [ZSM] (= semiflavus Thomson)

600 Bracon marshalli SZÉPLIGETI, 1901 [ZSM]

601 Bracon mediator NEES, 1834 [DE]

602 Bracon megapterus WESMAEL, 1838 [\# 86]

603 Bracon minutator (FABRICIUS, 1798) [DED] (= abscissor Nees; oestmaeli Wesmael; regularis Wesmael; rufigaster Szépligeti)

604 Bracon nigratus WESMAEL, 1838 [\# 62]

605 Bracon nigricollis WESMAEL, 1838 [\# 86]

606 Bracon nigriventris WESMAEL, 1838 [\# 64] (= fumigidus Szépligeti; indubius Szépligeti; laticeps Telenga; lautus Szépligeti; lencoranus Telenga; persimilis Telenga; sphaerocephalus Szépligeti) Remark: If $B$. sphaerocephalus is considered to be valid: also recorded from Germany (ZSM; det. Papp).

607 Bracon obscurator NEES, 1811 [DEM]

608 Bracon ocbropus NEES, 1834 [\# 86]

609 Bracon osculator NEES, 1811 [DED] (= bisignatus Wesmael; degenerator Marshall; temporalis Telenga; venustus Telenga)

610 Bracon pallicarpus THOMSON, 1892 [\# 83]

611 Bracon pallidator NEES, 1811 [\# 86]

612 Bracon palpebrator RATZEBURG, 1844 [\# 86]

613 Bracon pamicomis THOMSON, 1892 [ZSM] (= carbonarius Szépligeti) 
614 Bracon parulus WESMAEL, 1838 [ZSM] (= fumipennis Thomson; fuscipennis Thomson; thomsoni Marshall)

615 Bracon pectoralis WESMAEL, 1838 [\# 62] (= ochrosus Szépligeti)

616 Bracon pellucidus RATZEBURG, 1852 [\# 86]

617 Bracon peroculatus WESMAEL, 1838 [\# 86]

618 Bracon picticornis WESMAEL, 1838 [\# 86] (= gallarum Ratzeburg)

619 Bracon pineti THOMSON, 1892 [ZSM]

620 Bracon pliginskii TELENGA, 1936 [\# 86]

621 Bracon pratermissus MARSHALL, 1885 [ZSM]

622 Bracon punctifer THOMSON, 1892 [\# 86]

623 Bracon punctulator NEES, 1834 [\# 86]

624 Bracon pusillimus FAFRINGER, 1928 [\# 86] (= pusillus Ratzeburg)

625 Bracon ratzeburgii DALLA TORRE, 1898 [\# 86] (= longicaudis Ratzeburg)

626 Bracon rimulator NEES, 1834 [\# 86]

627 Bracon roberti WESMAEL, 1838 [\# 86]

628 Bracon scabriusculus DALLA TORRE, 1898 [\# 86] (= scaber Thomson)

629 Bracon schmiedeknechti FAHRINGER, 1927 [\# 86]

630 Bracon sinuatus NEES, 1834 [\# 86]

631 Bracon speerscbneideri SCHMIEDEKNECHT, 1896 [\# 86]

632 Bracon stabilis WESMAEL, 1838 [DET (= concolor Thomson; concolorans Marshall)

633 Bracon subglaber SZÉPLIGETI, 1901 [DEI] (= tauricus Telenga)

634 Bracon subrugosus SZÉPLIGETI, 1901 [ZSM]

635 Bracon telengai (MULJARSKAYA, 1955) [ZSM]

636 Bracon tener SZÉPLIGETI, 1904 [ZSM]

637 Bracon terebella WESMAEL, 1838 [ZSM] (= curticaudis Szépligeti)

638 Bracon thuringiacus SCHMIEDEKNECHT, 1896 [DEI]

639 Bracon titubans WESMAEL, 1838 [\# 86] (= tarsator Thomson)

640 Bracon tornator MARSHALL, 1885 [\# 40] (= aequalis Thomson)

641 Bracon triangularis NEES, 1834 [\# 86]

642 Bracon trucidator MARSHALL, 1898 [\# 86] (= minutator auct.)

643 Bracon trypetanus FAHRINGER, 1927 [\# 86]

644 Bracon urinator (FABRICIUS, 1798) [DEI] (= comptus Marshall)

645 Bracon variator NEES, 1811 [ZSM] (= breviventris Szépligeti; caudiger Thomson; collaris Telenga; collinus Szépligeti; explorator Szépligeti; hemiflavus Szépligeti; maculiger Wesmael; praecox Wesmael; pumilionis Roman) Remark: If $B$. maculiger and / or B. pumilionis are considered to be valid: also recorded from Germany (ZSM; det. Papp).

646 Bracon variegator SPINOLA, 1808 [DEI] (= melanosoma Szépligeti; natıulus Szépligeti)

647 Bracon vitripennis RATZEBURG, 1852 [\# 86]

648 Bracon xanthogaster NEES, 1834 [\# 40]

649 Ceratobracon stshegolevi TELENGA, 1933 [DED]

650 Coeloides abdominalis (ZETTERSTEDT, 1838) [ZSM]

651 Coeloides bostrichorum GIRAUD, 1872 [ZSM] 
652 Coeloides filiformis RATZEBURG, 1852 [ZSM] (= nigritorax Russo)

653 Coeloides forsteri HAESELBARTH, 1967 [ZSM] (= foersteri Haeselbarth misspell.)

654 Coeloides melanostigma STRAND, 1918 [ZSM] (= sordidator Ratzeburg auct.; stigmaticus Hellén) Remark: The currently usually used name "Coeloides sordidator" seems to be unjustified, as the description does not fit with Coeloides (EH).

655 Coeloides melanotus WESMAEL, 1838 [ZSM]

656 Coeloides rossicus (KOKUJEV, 1902) [DEI]

657 Coeloides scolyticida WESMAEL, 1838 [\# 29]

658 Coeloides strobilorum (RATZEBURG, 1848) [\# 86]

659 Coeloides ungularis THOMSON, 1892 [\# 29]

660 Cyanopterus extricator (NEES, 1834) [\# 86] (= brachyurus Telenga; brevicauda Thomson)

661 Cyanopterus flavator (FABRICIUS, 1793) [\# 86] (= barcinonensis Marshall; flavulator Ratzeburg; longipalpis Thomson)

662 Cyanopterus konowii (MARSHALL, 1897) [\# 86] (= konowii Marshall misspell.)

663 Cyanopterus nigrator (ZETTERSTEDT, 1838) [ZSM] (= borealis Hellén)

664 Ipbiaulax fastidiator (FABRICIUS, 1781) [\# 86] (= coccineus Brullé; fascidiator Fabricius misident.; multiarticulatus Ratzeburg)

665 Ipbiaulax impostor (SCOPOLI, 1763) [DEI] (= carissimus Shestakov; denigrator Fabricius; parvulus Shestakov; pulchellus Telenga)

666 Iphiaulax mactator (KLUG, 1817) [DEI]

667 Iphiaulax umbraculator (NEES, 1834) [\# 86]

668 Pigeria piger (WESMAEL, 1838) [DE] (= ornatulus Telenga)

669 Pseudovipio castrator (FABRICIUS, 1798) [\# 86]

670 Pseudovipio corsicus (MARSHALL, 1897) [\# 86] (= appellator Marshall)

671 Pseudovipio guttiventris (THOMSON, 1892) [DET] (= variegata Boheman)

672 Pseudovipio inscriptor (NEES, 1834) [\# 86]

673 Vipio appellator (NEES, 1834) [DET (= mendax Kokujev)

674 Vipio longicauda (BOHEMAN, 1853) [DED (= nominator Fabricius, 1793 not 1787)

675 Vipio terrefactor (VILLERS, 1789) [\# 86]

\section{Cardiochilinae}

676 Cardiocbiles saltator (FABRICIUS, 1781) [ZMHUB] (= brachialis Rondani; katkowi Kokujev; sibiricus Telenga)

\section{Cenocoeliinae}

677 Cenocoelius analis (NEES, 1834) [\# 86] (= flavifrons Haliday)

678 Cenocoelius secalis (LINNÉ, 1758) [\# 86] (= agricolorator Linné; femorator Tobias) Remark: The species belongs to the subgenus Lestricus (SB) that can be considered to be a valid genus (CvA).

\section{Charmontinae}

679 Charmon extensor (LINNÉ, 1758) [\# 02] (= brevicauda Hellén; cruentatus HALIDAY; gracilis Provancher; luteus Cameron; pectoralis Nees; pleuralis Cresson; striatus SHESTAKOV) Remark: CvA considers Cbarmon cruentatus HALIDAY, 1833 as a valid species (also recorded from Germany). 


\section{Cheloninae}

680 Ascogaster abdominator(DAHLBOM, 1833) [ZMHUB] (= femoralis Herrich-Schäffer; fulviventris Curtis; instabilis Wesmael; pallida Ruthe; rufiventris Herrich-Schäffer)

681 Ascogaster albitarsis REINHARD, 1867 [\# 86] (= albitarsus Reinhard misspell.; leptopus Thomson; similis Herrich-Schäffer)

682 Ascogaster anmularis (NEES, 1816) [ZSM]

683 Ascogaster armata WESMAEL, 1835 [\# 39] (= esenbeckii Curtis; luteicornis HerrichSchäffer; pulchellus Curtis)

684 Ascogaster bicarinata (HERRICH-SCHÄFFER, 1838) [\# 39] (= mlokossewitschi Kokujev; rufiventris Telenga)

685 Ascogaster bidentula WESMAEL, 1835 [ZSM] (= atamiensis Ashmead; fuscipennis Thomson; gibbiscuta Thomson; multiarticulatus Ratzeburg; rufipes sensu Reinhard; scabriusculus Zetterstedt)

686 Ascogaster brevicornis WESMAEL, 1835 [\# 39] (= monilicornis Herrich-Schäffer)

687 Ascogaster canifrons WESMAEL, 1835 [ZSM] (= zernyanus Fahringer)

688 Ascogaster consobrina CURTIS, 1837 [ZSM]

689 Ascogaster dentifer TOBIAS, 1976 [ZSM]

690 Ascogaster dispar FAHRINGER, 1934 [\# 40] (= spinifer Tobias)

691 Ascogaster excisa (HERRICH-SCHÄFFER, 1838) [ZMHUB] (= longiventris Tobias)

692 Ascogaster grahami HUDDLESTON, 1984 [DEI]

693 Ascogaster klugii (NEES, 1816) [ZSM] (= neesii Reinhard; ruficeps Wesmael)

694 Ascogaster quadridentata WESMAEL, 1835 [ZSM] (= carpocapsae Viereck; cynipum Thomson; egregius Kokujev; epinotiae Watanabe; impressus Herrich-Schäffer; nigrator Szépligeti; nigricornis Thomson; rufipes auct.)

695 Ascogaster rufidens WESMAEL, 1835 [ZSM] (= laevigator Ratzeburg; rufipes HerrichSchäffer)

696 Ascogaster rufipes (LATREILLE, 1809) [ZSM] (= elegans Nees; fasciatus Dahlbom; nigribasis Fahringer; pallipes Herrich-Schäffer; ratzeburgii Marshall; rugosulus Goureau; soror Telenga)

697 Ascogaster scabricula (DAHLBOM, 1833) [ZSM] (= clypealis Thomson; limitatus Wesmael)

698 Ascogaster varipes WESMAEL, 1835 [ZSM] (= sternalis Thomson)

699 Chelonus acuminatus HERRICH-SCHÄFFER, 1838 [\# 86]

700 Chelonus annulatus (NEES, 1816) [ZMHUB] (= decorus Marshall; fenestratus Nees)

701 Chelonus annulipes WESMAEL, 1835 [ZMHUB]

702 Chelonus antennalis THOMSON, 1874 [\# 40]

703 Chelonus bimaculatus SZÉPLIGETI, 1896 [\# 40] (= minor Fahringer; nigriventris Papp)

704 Chelonus canescens WESMAEL, 1835 [\# 86]

705 Chelonus carbonator MARSHALL, 1885 [ZMHUB]

706 Cbelonus corvulus MARSHALL, 1885 [ZMHUB] (= suturalis Szépligeti)

707 Cbelonus cylindrus (NEES, 1816) [ZMHUB] (= ebenius Fahringer; speculator Marshall; variabilis Herrich-Schäffer) Remark: See comments under Cbelonus oculator.

708 Chelonus elongatus SZÉPLIGETI, 1898 [\# 86]

709 Cbelonus inanitus (LinNÉ, 1767) [\# 86] (= atomos Rossi)

710 Cbelonus nigrinus RATZEBURG, 1844 [\# 86] 
711 Cbelonus obscuratus HERRICH-SCHÄFFER, 1838 [ZMHUB] (= intermedius Thomson; nigrifemur Papp)

712 Chelonus oculator (FABRICIUS, 1775) [\# 86] (= integer von Block; mutabilis Nees; oculator sensu Panzer ?) Remark: It is not certain, if oculator sensu PANZER is the same as oculator FABRICIUS. On the base of much material it seems that oculator is a rather variable and very common species. There should be a revision of the genus first to decide about the synonymy. Perhaps Chelonus cylindrus belongs to oculator, too ( $\mathrm{C} \vee \mathrm{A})$.

713 Chelonus olgae KOKUJEV, 1895 [\# 86] (= schmiedeknechti Szépligeti)

714 Chelonus pictipes WESMAEL, 1838 [\# 40]

715 Chelonus productus HERRICH-SCHÄFFER, 1838 [\# 86] (= submuticus auct.) Remark: There are no records of the true Cb. submuticus WESMAEL, 1835 (=luteipes Thomson) from Germany.

716 Chelomus pusio MARSHALl, 1885 [\# 86]

717 Chelonus rimosus HERRICH-SCHÄFFER, 1838 [\# 86]

718 Chelonus scabrator (FABRICIUS, 1793) [DET (= buccatus Thomson; humilis Thomson; rugigena Thomson; scaber Nees)

719 Cbelonus zimini TOBIAS, 1972 [\# 79] Remark: Perhaps a pale form of Cbelonus elongatus SZÉPLIGETI, 1898 (cf. PAPP, 1995 [\# 79]).

720 Microchelonus atripes (THOMSON, 1874) [\# 76] (= kamtshaticus Tobias)

721 Microcbelonus basalis (CURTIS, 1837) [\# 86]

722 Microcbelonus contractus (NEES, 1816) [ZMHUB]

723 Microcbelonus erosus (HERRICH-SCHÄFFER, 1838) [\# 36] Remark: According to PAPP (1975 [\# 95]) a valid species. Often considered to belong to $M$. retusus.

724 Microcbelonus exilis (MARSHALL, 1885) [\# 86]

725 Microcbelonus fenestratus (NEES, 1816) [\# 86] (= basalis sensu Telenga)

726 Microcbelonus gravenhorstii (NEES, 1816) [ZMHUB] (= adjaricus Tobias; gravenhorsti Wesmael; maculator Dahlbom; mandibularis Haliday; tricolor Tobias)

727 Microcbelonus lugubris (WESMAEL, 1835) [\# 86]

728 Microcbelonus micropbtalmus (WESMAEL, 1838) [\# 86] ( = microphthalmus Wesmael misspell.)

729 Microchelonus minutus (COSTA, 1884) [\# 86]

730 Microcbelonus nigritulus (DAHLBOM, 1832) [\# 77]

731 Microcbelonus parcicornis (HERRICH-SCHÄFFER, 1838) [ZMHUB] (= rectus Papp)

732 Microcbelonus pedator (DAHLBOM, 1832) [\# 40] (= secutor Marshall)

733 Microcbelonus pellucens (NEES, 1816) [\# 86] (= alboannulatus Szépligeti; nitens Reinhard; pulchricornis Szépligeti)

734 Microcbelonus retusus (NEES, 1816) [ZMHUB] (= caudatus Thomson; emarginatus HerrichSchäffer; pamiricus Voinovskaja-Kriger; subemarginatus Herrich-Schäffer)

735 Microchelonus risorius (REINHARD, 1867) [ZMHUB] (= fissuralis Tobias)

736 Microchelonus striatiscuta (FAHRINGER, 1934) [\# 86]

737 Microcbelonus subsulcatus (HERRICH-SCHÄFFER, 1838) [\# 86]

738 Microcbelonus sulcatus (JURINE, 1807) [\# 86]

739 Phanerotoma acuminata SzÉPLIGETI, 1908 [ZSM]

740 Phanerotoma bilinea LYLE, 1924 [\# 07] (= gregori Šnoflak) 
741 Pbanerotoma dentata (PANZER, 1805) [ZSM] (= bilinea sensu Telenga; flavescens Fahringer; minor Šnoflak; rendiles Fahringer)

742 Phanerotoma planifrons (NEES, 1816) [\# 86] (= asini Llopsis; bicolor Šnoflak; blanda Fahringer; maderi Fahringer; snoflaki Shenefelt)

743 Pbanerotoma rufescens (LATREILLE, 1809) [\# 40] (= planifrons auct.; platypyga Šnoflak; rugiferum Wesmael)

744 Phanerotomella bisulcata (HERRICH-SCHÄFFER, 1838) [\# 07] (= flavipes Šnoflak; nigxa Szépliget)

\section{Dirrhopinae}

745 Dirrhope rufa FÖRSTER, 1851 [ZMHUB]

\section{Doryctinae}

746 Caenopbanes incompletus (RATZEBURG, 1844) [DEI] (= tetropis Fischer)

747 Dendrosoter curtisii (RATZEBURG, 1848) [\# 86]

748 Dendrosoter hartigii (RATZEBURG, 1848) [DE] (= caenopachoides Ruschka; flaviventris Förster; hartigi Ratzeburg misspell.) Remark: CvA considers Dendrosoter flaviventris FÖRSTER, 1878 (= caenopachoides Ruschka) a valid species (also recorded from Germany).

749 Dendrosoter middendorffi (RATZEBURG, 1848) [ZSM]

750 Dendrosoter protuberans (NEES, 1834) [DET] (= incompletus Thomson; insignis Förster)

751 Doryctes fuscatus (NEES, 1834) [\# 86]

752 Doryctes germanicus BELOKOBYLSKI, 2001 [DEI]

753 Doryctes beydenii REINHARD, 1865 [ZMHUB] (= heydeni Reinhard misspell.)

754 Doryctes leucogaster (NEES, 1834) [ZSM] (= caucasica Kokujev, disputabilis Kokujev; erythrogaster Wesmael; liogaster Marshall; marothiensis Szépligeti; pulchripes Szepligeti; turkestanicus Telenga)

755 Doryctes nobilis (NEES, 1834) [\#86]

756 Doryctes pomarius REINHARD, 1865 [ZMHUB]

757 Doryctes striatellus (NEES, 1834) [DEI] (= brachyurus Marshall; mutillator auct.; obliteratus Nees; petrovskii Kokujev; rex Marshall; striatelloides Strand; strigatus Kokujev; tabidus Haliday) Remark: The widely used name "Doryctes mutillator (THUNBERG, 1822)" is based on Ichneumon mutillator THUNBERG, 1824, which is a replacement name or an unjustified emendation for Ichneumon mutillarius FABRICIUS, 1775 (see HORSTMANN, 1999 [\# 93]). I. mutillarius is considered to be a junior subjective synonym of Spatbius exarator (LINNAEUS, 1758). The species hitherto called Doryctes mutillator is to be named Doryctes striatellus. CvA considers D. obliteratus (NEES, 1834) to be valid (also recorded from Germany).

758 Doryctes undulatus (RATZEBURG, 1852) [ZSM]

759 Ecpbylus silesiacus (RATZEBURG, 1848) [ZSM] (= beltrani Docavo; carinatus Hedqvist; chaetoptelii Gautier \& Russo; eccoptogastri Ratzeburg; hylesini Ratzeburg; minutissimus Ratzeburg; pinicola Hedqvist; sileseacus Ratzeburg misspell.) Remark: There are good reasons (e.g. differences in biology) to suppose that several of the synonyms are valid species (CvA).

Hecabolus sulcatus CURTIS, 1834 [ZSM] (= belgicum Wesmael)

761 Heterospilus ater FISCHER, 1960 [ZSM]

762 Heterospilus austriacus (SZÉPLIGETT, 1906) [\# 40] (= incompletus auct.)

763 Heterospilus caesus (NEES, 1834) [\# 86]

764 Heterospilus ceplizi ROHWER, 1925 [ZSM] (= basifurcatus Fischer; rubicundus Fischer; testaceus Telenga) 
765 Heterospilus hemipterus (THOMSON, 1892) [ZMHUB]

766 Heterospilus mbicola FISCHER, 1968 [SCHNEE] (= tobiasi Belokobylskij)

767 Heterospilus tanricus TELENGA, 1941 [ZMHUB] (= graeffei Fischer)

768 Hypodoryctes sibiricus KOKUJEV, 1900 [ZSM]

769 Monolexis foersteri MARSHALL, 1897 [\# 86]

770 Monolexis fuscicomis FörSTER, 1862 [ZMHUB] (= atis Nixon; doderoi Mantero; lavagnei Picard)

771 Ontsira antica (WOLLASTON, 1858) [ZSM] (= caudalis Hellén; gallicus Reinhard; incertus Ashmead)

772 Ontsira imperator (HALIDAY, 1836) [DEI] (= cingulatus Provancher; ignea Ratzeburg; praecisus Ratzeburg; zonatus Wesmael) Remark: O. ignea may be a valid species (CVA).

773 Ontsira longicaudis (GIRAUD, 1857) [DE] (= ustulata Fahringer)

774 Ontsira spathizfornis (RATZEBURG, 1848) [ZMHUB] (= maculipennis Szépligeti)

775 Polystenus rugosus FÖRSTER, 1862 [ZMHUB] (= aciculatus Reinhard)

776 Rhaconotus acioulatus RUTHE, 1854 [BMNH]

777 Rbaconotus elegans (FÖRSTER, 1862) [ZMHUB]

778 Rbaconotus pictipennis (REINHARD, 1885) [ZMHUB]

779 Rhoptrocentrus piceus MARSHALL, 1897 [DED (= reinhardi Fahringer; syrmiensis Szépligeti)

780 Spatbius brevicaudis RATZEBURG, 1844 [\# 86]

781 Spathius curvicaudis RATZEBURG, 1844 [DED] (= curvicaudus Ratzeburg misspell.)

782 Spatbius erythrocephalus WESMAEL, 1838 [ZSM]

783 Spatbius exarator (LINNÉ, 1758) [ZSM] (= clavatus Panzer; exannulatus Ratzeburg; mutillator Thunberg; mutillarius Fabricius; strandi Fahringer)

784 Spatbius lignarius (RATZEBURG, 1852) [\# 86] Remark: May be synonym of S. brevicaudis RATZEBURG, 1844 (CvA).

785 Spatbius pedestris WESMAEL, 1838 [ZMHUB] (= apterus Wesmael)

786 Spatbius polonicus NIEZABITOWSKI, 1910 [ZMHUB]

787 Spatbius radrayanus RATZEBURG, 1848 [ZSM]

788 Spatbius rubidus (ROSSI, 1794) [DE] (=umbratus Trentepohl)

\section{Euphorinae}

789 Allurus lituratus (HALIDAY, 1835) [\# 86]

790 Allurus muricatus (HALIDAY, 1833) [DED (= armatus Wesmael; nigra Lyle)

791 Aridelus egregius (SCHMIEDEKNECHT, 1907) [ZMHUB] (= nigricans Chao)

792 Blacometeorus brevicauda (HELLÉN, 1958) [RMNH]

793 Blacus armatulus RUTHE, 1861 [BMNH]

794 Blacus conformis WESMAEL, 1835 [ZSM]

795 Blacus diversicomis (NEES, 1834) [ZSM] (= compar Ruthe)

796 Blacus errans (NEES, 1812) [ZSM] (= vagans Ruthe)

797 Blacus exilis (NEES, 1812) [ZMHUB] (= pallipes Förster; propallipes Shenefelt)

798 Blacus filicomis HAESELBARTH, 1973 [\# 04]

799 Blacus fischeri HAESELBARTH, 1973 [ \# 04]

800 Blacus bastatus HALIDAY, 1835 [\# 04] (= terebrator Ruthe) 
801 Blacus bostilis HAESELBARTH, 1973 [ZMHUB]

802 Blacus bumilis (NEES, 1812) [ZSM] (= cerealis Curtis; trivialis Haliday)

803 Blacus instabilis RUTHE, 1861 [\# 04]

804 Blacus interstitialis RUTHE, 1861 [BMNH] (= ascinellae Fischer)

805 Blacus leptostigma RuTHE, 1861 [BMNH]

806 Blacus longipennis (GRAVENHORST, 1809) [ZSM] (= dubius Ruthe)

807 Blacus macropterus HAESELBARTH, 1973 [ZSM]

808 Blacus maculipes WESMAEL, 1835 [ZSM]

809 Blacus mamillanus RUTHE, 1861 [BMNH] (= aptenodytes Marshall)

810 Blacus modestus HAESELBARTH, 1973 [ZSM]

811 Blacus paganus HALIDAY, 1835 [ZSM] (= brevicornis Ruthe)

812 Blacuspallipes HALIDAY, 1835 [ZSM] (= barynoti auct; tuberculatus Wesmael)

813 Blacus robustus HAESEIBARTH, 1973 [\# 04]

814 Blacus rufescens RUTHE, 1861 [BMNH] (- spinifer Thomson)

815 Blacus ruficomis (NEES, 1812) [ZSM] (= tipulator Zetterstedt)

816 Blacus stelfoxi HAESELBARTH, 1973 [\# 04]

817 Blacus strictus STELFOX, 1941 [\# 04]

818 Blacus tripudians HALIDAY, 1835 [\# 04]

819 Centistes ater (NEES, 1834) [DEI] ( = excrucians Haliday; lativalvis Jakimavicius)

820 Centistes uuspidatus (HALIDAY, 1833) [DE] (= lucidator Nees)

821 Centistes edentatus (HALIDAY, 1835) [DET (= edentulus Haliday misspell.)

822 Centistes fuscipes (NEES, 1834) [DEM]

823 Centistes nasutus (WESMAEL, 1838) [ZMHUB] (= saxo Reinhard)

824 Centistes paupella (SHENEFELT, 1969) [DET ( = pallipes Wesmael)

825 Centistes rufithorax (TELENGA, 1950) [\# 32]

826 Centistes symmi FERRIËE, 1954 [\# 78]

827 Cosmophorus klugii RATZEBURG, 1848 [\# 86]

828 Dinocanpus coccinellae (SCHRANK, 1802) [DEI] (= terminatus Nees)

829 Elasmosoma berolinense RUTHE, 1858 [\# 86]

830 Leiophron accincta (HALIDAY, 1835) [\# 44] (= laeviventris Ruthe) Remark: Microctonus laeviventris RUTHE, 1856 (described from Germany) is a hitherto unpublished synonym (CvA). The species belongs to the subgenus Peristenus (SB) that can be considered a valid genus (CvA). The generic name Leiopbron is from "smooth brain" in Greek and is feminine (CvA), therefore the species names are to be changed accordingly.

831 Leiophron apicalis HALIDAY, 1833 [\# 86]

832 Leiophron brevicomis (HERRICH-SCHÄFFER, 1838) [\# 86] Remark: The species belongs to the subgenus Peristenus (SB) that can be considered a valid genus (CvA).

833 Leiopbron deficiens (RUTHE, 1856) [\# 44]

834 Leiophron duplobrevicornis SHENEFELT, 1969 [\# 86] (= brevicornis Ruthe) Remark: The species belongs to the subgenus Peristenus (SB) that can be considered a valid genus (CvA).

835 Leiophron duploclaviventris SHENEFELT, 1969 [\# 86] (= brevicornis sensu Ruthe; claviventris Ruthe; ruthei Loan) Remark: The species belongs to the subgenus Euphorus (SB) that can be considered a valid genus ( $\mathrm{CVA}$ ). 
836 Leiopbron fascipennis (RUTHE, 1856) [DEI] (= fasciipennis Ruthe misspell.)

837 Leiophron grandiceps (THOMSON, 1892) [DET (= orchesiae auct.) Remark: The species belongs to the subgenus Peristenus (SB) that can be considered a valid genus (CVA).

838 Leiopbron nitida CURTIS, 1833 [\# 86] Remark: The species belongs to the subgenus Peristenus (SB) that can be considered a valid genus (CvA).

839 Leiopbron oblita (RUTHE, 1856) [\# 86] Remark: The species is not a synonym of L. similis (CvA). It belongs to the subgenus Euphorus (SB) that can be considered a valid genus (CvA).

840 Leiophron pallidistigma CURTIS, 1833 [DEI] (= claviventris Wesmael; intactus Haliday; pallicornis Nees; parvulus Ruthe) Remark: The species belongs to the subgenus Euphorus (SB) that can be considered a valid genus (CvA).

841 Leiopbron pallipes CURTIS, 1833 [DET] (= barbiger Wesmael; nocturnus Viereck; punctatus Provancher; tuberculifer Marshall) Remark: The species belongs to the subgenus Peristenus (SB) that can be considered a valid genus (CvA).

842 Leiopbron picipes CURTIS, 1833 [DEI] (= coactus Marshall) Remark: The species belongs to the subgenus Peristenus (SB) that can be considered a valid genus (CvA).

843 Leiophron reclinator (RUTHE, 1856) [\# 44] Remark: The species belongs to the subgenus Peristenus (SB) that can be considered a valid genus (CvA).

844 Leiophron relicta (RUTHE, 1856) [\# 44] (= stygicus Loan) Remark: The species belongs to the subgenus Peristents (SB) that can be considered a valid genus (CvA).

845 Leiophron rubricollis (THOMSON, 1892) [DED] ( $=$ reclinator sensu Tobias) Remark: The species belongs to the subgenus Peristenus (SB) that can be considered a valid genus (CvA).

846 Leiopbron similis CuRTIS, 1833 [\# 44] (= basalis Curtis) Remark: The species belongs to the subgenus Euphorus (SB) that can be considered a valid genus (CvA). Leiopbron basalis CURTIS, 1833 is an unpublished synonym (CvA).

847 Leiopbron truncator (RUTHE, 1856) [\# 44] Remark: The species belongs to the subgenus Eupborus (SB) that can be considered a valid genus ( $\mathrm{CvA}$ ).

848 Marshiella plumicornis (RUTHE, 1856) [\# 86]

849 Meteorus abdominator (NEES, 1811) [DEI] (= brevipes Wesmael misident.; brevipesalis Shenefelt; brunnipes Ruthe)

850 Meteorus abscissus THOMSON, 1895 [\# 85] (= pulchricornis sensu Ruthe)

851 Meteorus affinis (WESMAEL, 1835) [\# 38] (= gracilis Ruthe; punctiventris Ruthe; ruthei Schmiedeknecht; voloscensis Fischer)

852 Meteorus brevicanda THOMSON, 1895 [DE] (= mongolicus Fahringer; thuringiacus Schmiedeknecht)

853 Meteorus cespitator (THUNBERG, 1822) [DEI] (= ambiguus Ruthe; atrator Curtis; cespidator Thunberg misspell; humeralis Zetterstedt; microcerus Wesmael; rufipes Zetterstedt; similator Nees)

854 Meteorus cinctellus (SPINOLA, 1808) [DET (= fuscipes Wesmael; necator Trentepohl; tenellus Marshall)

855 Meteorus cis (BOUCHÉ, 1834) [\# 86] (= profligator Haliday) Remark: Perilitus profligator HALIDAY, 1835 is an unpublished synonym of $M$. cis (CvA).

856 Meteorus colon (HALIDAY, 1835) [DET] (= alternata Ruthe; continua Ruthe; fasciatus Ratzeburg; fragilis Haliday; fragilis Wesmael; luridus Ruthe; pallida Ruthe; trivittata Ruthe)

857 Meteorus consimilis (NEES, 1834) [DEI] (= albicornis Ruthe; brevipes Wesmael; flagellatus Alexeev) 
858 Meteorus corax MARSHALL, 1898 [\# 38] (= monochami Fischer; pospelovi Telenga; pospelowi Telenga misspell.)

859 Meteorus eadyi HUdDLESTON, 1980 [\# 38]

860 Meteorus filator (HALIDAY, 1835) [DEI] (= hodisensis Fischer; laticeps Wesmael)

861 Meteorus beliopbilus FISCHER, 1970 [DET] (= luridus sensu Tobias)

862 Meteorus hirsutipes HUDDLESTON, 1980 [\# 38]

863 Meteorus ictericus (NEEs, 1812) [DED] (= adoxophyesi Minamikawa; confinis Ruthe; consors Ruthe; crassicrus Thomson; ephippium Curtis; fallax Ruthe; icterius Nees misspell; liquis Ruthe; lophyriphagus Fahringer; lucidator Trentepohl; makinoharanus Minamikawa; minutor Thunberg; pleuralis Ruthe; rubriceps Ratzeburg; xanthomelas Wesmael)

864 Meteorys jasulator (HALIDAY, 1835) [\# 38] (= obscurellus Ruthe; tenuicornis Thomson)

865 Meteorus lionotus THOMSON, 1895 [DED] (= ruficoloratus Fischer)

866 Meteorus longicaudis (RATZEBURG, 1848) [\# 38]

867 Meteorus melanostictus CAPRON, 1887 [\# 40] (= niger Lyle)

868 Meteorus micropterus (HALIDAY, 1835) [DEI]

869 Meteorus obfuscatus (NEES, 1812) [DET (= fodori Papp; formosus Wesmael; orchesiae Boie; thoracicus Curtis)

870 Meteorus obsoletus (WESMAEL, 1835) [DEI] (= viridanae Johannson)

871 Meteorus oculatus RUTHE, 1862 [\# 38] (= pachypus Schmiedeknecht)

872 Meteorus pallipes (WESMAEL, 1835) [\# 38] (= nigritarsis Ruthe) Remark: HUDDLESTON (1980 [ 38]) considered ruficeps as nomen dubium. $M$. pallipes is a junior synonym of $M$. ruficeps (NEES, 1834) according to PAPP (1983 [\# 98])

873 Meteorus pendulator (LATREILLE, 1799) [DED] (= gyrator Thunberg; parvulus Thomson; petiolator Zetterstedt; scutellator Nees; unicolor sensu Ruthe) Remark: HUDDLESTON (1980 [ 38]) considered pendulator as a nomen dubium. Icbneumon pendulator is the type species of Meteorus and CvA will soon publish a note on its synonymy.

874 Meteorus pulcbricornis (WESMAEL, 1835) [DEI] (= graeffei Fischer; japonicus Ashmead; macedonicus Fischer; nipponensis Viereck; striatus Thomson; thomsoni Marshall; tuberculifer Fischer)

875 Meteoris rubens (NEES, 1812) [DE] (= coquilleti Ashmead; heteroneurus Thomson; islandicus Ruthe; laeviventris Wesmael misspell.; leviventris Wesmael; mamestrae Viereck; medianus Ruthe; mellinervis Viereck; mesopotamicus Fischer; scutatus Costa; szechuanensis Fahringer; vulgaris Cresson)

876 Meteorus rufus (DE GEER, 1773) [DET] (= chinensis Holmgren; unicolor Wesmael)

877 Meteorus salicorniae SCHMIEDEKNECHT, 1897 [\# 38] (= ocellatus Watanabe)

878 Meteorus sulcatus SZÉPLIGETI, 1896 [\# 38] (= insignis Muesebeck; molorchi Fischer)

879 Meteorus tabidus (WESMAEL, 1835) [DET] (= dubius Ruthe; facialis Ruthe; pentheri Fischer)

880 Meteorus versicolor (WESMAEL, 1835) [DET (= bimaculatus Wesmael; brevicornis Ratzeburg; camptolomae Watanabe; decoloratus Ruthe; hartigi Shenefelt; ikonomovi Fischer; rugator Ratzeburg)

881 Myiocephalus boops (WESMAEL, 1835) [ZMHUB] (= falconivibrans Morley; longipes Förster)

882 Neoneurus auctus (THOMSON, 1895) [ZSM] (= halidaii Marshall; bistigmaticus Morley)

883 Neoneurus clypeatus (FöRSTER, 1862) [\# 86] (= viennensis Giraud) Remark: The synonymy was published in a curious way by SHAW (1992 [\# 97]) in the revision of Neoneurus. He cites CvA's label on the FÖRTER type (with the synonymy) and comments on this. He claims that the male holotype of FÖRSTER is slightly different and postpones synony- 
mization till more material is known from the (Aachen) area. Now CVA has seen material from the Dutch side of the border and it seems that there is really only one species (CvA).

884 Perilitus aemulus (RUTHE, 1856) [\# 31] Remark: The species belongs in the subgenus Townesilitus (SB) that can be considered to be a valid genus (EH, CvA).

885 Perilitus aethiopoides (LOAN, 1975) [\# 40] (= aethiops auct.)

886 Perilitus aethiops NEES, 1834 [\# 86]

887 Perilitus areolaris GERDIN \& HEDQVIST, 1985 [DED]

888 Perilitus bicolor (WESMAEL, 1835) [DEI] Remark: The species belongs in the subgenus Tomnesilitus (SB) that can be considered to be a valid genus (EH, CvA).

889 Perilitus brevicollis HALIDAY, 1835 [\# 86]

890 Perilitus brevipetiolatus THOMSON, 1892 [ZSM]

891 Perilitus cerealium HALIDAY, 1835 [\# 86] (= rufipes Herrich-Schäffer; secalis sensu Haliday)

892 Perilitus colesi (DREA, 1968) [ZSM]

893 Perilitus consuetor NEES, 1834 [\# 86]

894 Perilitus cornelii HAESELBARTH, 1999 [\# 34]

895 Perilitus debilis WOLLASTON, 1858 [ZSM]

896 Perilitus dubius (WESMAEL, 1838) [DET]

897 Perilitus falciger (RUTHE, 1856) [\# 86]

898 Perilitus foveolatus REINHARD, 1862 [\# 34] (= sicheli Giard)

899 Perilitus fulviceps (RUTHE, 1856) [DEI] Remark: The species belongs in the subgenus Townesilitus (SB) that can be considered to be a valid genus ( $\mathrm{EH}, \mathrm{CvA}$ ).

900 Perilitus labilis (RuTHE, 1856) [\# 86] (= latibilis Meyer; latibsis Meyer)

901 Perilitus lancearius (RUTHE, 1856) [\# 86]

902 Perilitus melanopus (RUTHE, 1856) [BMNH]

903 Perilitus parcicornis (RUTHE, 1856) [BMNH]

904 Perilitus peregrinus HERRICH-SCHÄFFER, 1838 [\# 86]

905 Perilitus regius HAESELBARTH, 1999 [ 34]

906 Perilitus retusus (RUTHE, 1856) [BMNH] Remark: The species is according to the type not a synonym of $P$. brevicollis HALIDAY, but a valid species (EH).

907 Perilitus mitus (NEES, 1812) [\#34]

908 Perilitus stelleri (LOAN, 1972) [ZSM]

909 Perilitus vittatae (MUESEBECK, 1936) [ZSM]

910 Pygostolus falcatus (NEES, 1834) [ZMHUB] (= otiorhynchi Boudier)

911 Pygostolus multiarticulatus (RATZEBURG, 1852) [ZSM]

912 Pygostolus sticticus (FABRICIUS, 1798) [ZSM] (= barynoti Boudier; gigas Wesmael; sticticator Thunberg)

913 Rilipertus facialis (THOMSON, 1892) [\#33]

914 Rilipertus intricatus (RUTHE, 1859) [\# 33]

915 Rilipertus vaginator (WESMAEL, 1835) [\# 33]

916 Ropalophorus clavicornis (WESMAEL, 1835) [\# 41]

917 Streblocera flaviceps (MARSHALL, 1898) [ 86]

918 Streblocera fulviceps WESTWOOD, 1833 [DET] 
919

920

921

922

923

924

925

926

927

928

929

930

931

932

933

934

935

936

937

938

939

Streblocera macroscapus (RUTHE, 1856) [DEI]

Syniretus conterminus (NEES, 1834) [\# 86]

Syntretus elegans (RUTHE, 1856) [\# 86]

Syntretus fuscicoxis VAN ACHTERBERG \& HAESELBARTH, 2003 [ZSM]

Syntretus idalizus (HALIDAY, 1833) [ZSM] (= vernalis Wesmael; cultus Marshall)

Syntretus klugï (RUTHE, 1856) [\# 86]

Syntretus minimus VAN ACHTERBERG \& HAESELBARTH, 2003 [ZSM]

Syntretus ocularis VAN ACHTERBERG \& HAESELBARTH, 2003 [ZSM]

Syntretusparwicornis (RUTHE, 1862) [\# 86]

Syntretus politus (RUTHE, 1856) [BMNH] (= cynthius Lyle)

Syntretus pusio (MARSHALL, 1898) [ZSM]

Syntretus stenochora VAN ACHTERBERG \& HAESELBARTH, 2003 [ZSM]

Syntretus taegeri VAN ACHTERBERG \& HAESELBARTH, 2003 [DEI]

Syntretus xanthocephalus (MARSHALL, 1887) [ZSM] (= lyctaca Cole)

Syntretus quijleni VAN ACHTERBERG \& HAESELBARTH, 2003 [ZSM]

Syrrbizus delusorius FÖRSTER, 1862 [ZMHUB]

Wesmalia petiolata (WOLLASTON, 1858) [ZMHUB] (= asiatica Shestakov; cremasta Marshall; pendula Förster)

Zele albiditarsus CURTIS, 1832 [DEI] (= albiditarsis Curtis misspell.; albitarsus Haliday; albitarsus Nees; calcitrator Curtis; dispar Wesmael; maximus Muesebeck; pallitarsis Cresson; romani Fahringer; rufulus Thomson; testaceator Curtis; testatrix Schulz; wesmaeli Boie)

Zele caligatus (HALIDAY, 1835) [DEI] (= neesii Ruthe; sibiricus Fahringer)

Zele chlorophthalmus (SPINOLA, 1808) [DET] (= chrysophthalmus Nees; nigricollis Thomson; nudator Thunberg; splendens Costa)

Zele deceptor (WESMAEL, 1835) [DEI] (= reticulatus Muesebeck; separandus Fischer)

\section{Exothecinae}

940 Acrisis clavipes MarshaLL, 1888 [\# 86]

941 Acrisis fuscipes HELLÉN, 1957 [\# 41]

942 Acrisis gracilicornis FÖRSTER, 1862 [ZMHUB]

943 Avga opaca (HELLÉN, 1957) [ZMHUB] (= europaeica Tobias)

944 Cbremylus elaphus HALIDAY, 1833 [DEI] (= japonicus Ashmead; nigriceps Ashmead; rubiginosus Nees; terminalis Ashmead; tineavora Nagamori; transversus Say)

945 Clinocentrus cunctator (HALIDAY, 1836) [ZSM] (= analis Wesmael; gracilipes Thomson)

946 Clinocentrus excubitor (HALIDAY, 1836) [ZSM] (= marginellus Wesmael)

947 Clinocentrus exsertor (NEES, 1812) [ZSM] (= excubitor sensu Telenga; jaroshevskyi Telenga; orbitator Nees; striolatus Thomson)

948 Clinocentris wmbratilis HALIDAY, 1833 [ZSM] (= petiolaris Thomson)

949 Clinocentrus vestigator (HALIDAY, 1836) [ZSM]

950 Colastes abnormis (WESMAEL, 1838) [ZMHUB] (= glabricollis Thomson)

951 Colastes braconius HALIDAY, 1833 [DEI]

952 Colastes catenator (HALIDAY, 1836) [ZMHUB]

953 Colastes flavitarsis (THOMSON, 1892) [ZMHUB] 
954 Colastes foveolator (THOMSON, 1892) [\# 86]

955 Colastes fragilis (HALIDAY, 1836) [ZMHUB] (= semeyticus Jakimavicius)

956 Colastes bungaricus (SZÉPLIGETI, 1906) [ZMHUB] (= sculptiventris Tobias)

957 Colastes incertus (WESMAEL, 1838) [ZMHUB]

958 Colastes laevis (THOMSON, 1892) [ZSM] (=lissogaster Tobias) Remark: SB synonymized C. lissogaster TOBIAS, 1986 after examining of a rather long series of laevis. CvA considers lissogaster a valid species, despite the synonymisation by BELOKOBYLSKIJ (German specimen in RMNH, Leiden).

959 Colastes laticarpus (THOMSON, 1892) [\# 86] (= flaviventris Thomson)

960 Colastes lustrator (HALIDAY, 1836) [DEI] (= thomsoni Szépligeti)

961 Colastes magdalenae STERZYNSKI, 1983 [ZMHUB]

962 Colastes moldavicus TOBIAS, 1986 [ZMHUB]

963 Colastes pubicornis (THOMSON, 1892) [ZMHUB]

964 Colastes vividus PAPP, 1975 [SCHNEE]

965 Dimeris mira RUTHE, 1854 [ZSM]

966 Hormius antefurcalis BELOKOBYLSKIJ, 1995 [DED]

967 Hormitus moniliatus (NEES, 1812) [ZMHUB] (= piciventris Wesmael) Remark: Hormius piciventris WESMAEL, 1838 might be a valid species, but a revision is needed to decide the problem (also recorded from Germany) ( $\mathrm{C} v \mathrm{~A})$.

968 Lysitermus pallidus FörSTER, 1862 [ZMHUB] (= talitzkii Tobias)

969 Noserus facialis FÖRSTER, 1862 [ZMHUB] (= brevicauda Tobias)

970 Pambolus biglumis (HALIDAY, 1836) [\# 86] (= dubius Marshall; imminens Marshall; rosenhaueti Ratzeburg)

971 Pambolus tricolor (RUTHE, 1854) [BMNH]

972 Phanodus pallipes FöRSTER, 1862 [ZMHUB] (= chalveri Docavo; flavipes Förster; pallidipes Marshall; rufigaster Dahl)

973 Pbaenodus rugulosus HELLÉN, 1927 [BMNH]

974 Proacrisis acutus TOBIAS, 1983 [DEI]

975 Rhysipolis decorator (HALIDAY, 1836) [ZSM] (= caudatus Thomson; ruficeps Wesmael)

976 Rbysipolis bariolator (HALIDAY, 1836) [DEI]

977 Rhysipolis meditator (HALIDAY, 1836) [ZSM] (= bianchii Telenga; decorator auct.; flavicoxa Thomson misspell.; gigas Tobias; intermedius Wesmael; major Szépligeti; obscuripes Thomson; similis Szépligeti; variabilis Szépligeti; varicoxa Thomson) Remark: It seems to be possible that Rbysipolis varicoxa (THOMSON, 1892) represents a valid species that prefers warm dry habitats. Specimen from Germany in ZSM (CvA).

\section{Gnamptodontinae}

978 Gnamptodon decoris (FÖRSTER, 1862) [ZMHUB] (= bachmaieri Fischer; klemensiewiczii Niezabitowski)

979 Gnamptodon pumilio (NEES, 1834) [\# 86]

\section{Helconinae}

980 Aspicolpus carinator (NEES, 1812) [DEI]

981 Aspigonus flavicomis (NEES, 1834) [DEI] (= diversicornis Wesmael) Remark: Aspigonus diversicomis WESMAEL, 1835 is an unpublished synonym of flavicomis (CvA). 
982 Baeacis abietis (RATZEBURG, 1844) [DED]

983 Baeacis dissimilis (NEES, 1834) [\# 86] (= contractus Ratzeburg) Remark: The species seems to be better placed in Aspicolpus (CvA).

984 Baeacis intermedia FÖRSTER, 1878 [ZMHUB]

985 Diospilus capito (NEES, 1834) [DET]

986 Diospilus dispar (NEES, 1811) [ZMHUB] (= ephippium Nees; filator Nees)

987 Diospilus fusciventris HELLÉN, 1958 [DEI] (= longicauda Tobias)

988 Diospilus inflexus REINHARD, 1862 [DED] (= ovatus Marshall)

989 Diospilus kokujevi TOBIAS, 1986 [\# 81] Remark: It seems to be possible that the reference is based on a misidentification (CvA).

990 Diospilus melanoscelus (NEES, 1834) [\# 86]

991 Diospilus molorcbicola FISCHER, 1966 [\# 81]

992 Diospilus morosus REINHARD, 1862 [DE]

993 Diospilus nigricornis (WESMAEL, 1835) [DED (= affinis Wesmael; rufipes Reinhard) Remark: Diospilus affinis (WESMAEL, 1835) seems to be valid. Both species occur in the Netherlands in completely different habitats and are also habitually different. A German specimen is present in the RMNH (CvA).

994 Diospilus oleraceus HALIDAY, 1833 [\# 86] (= ruficornis Szépligeti)

995 Diospilus robustus REINHARD, 1862 [\# 86]

996 Diospilus rugosus THOMSON, 1895 [\# 86] Remark: Originally described from "norra Tyskland" (Northern Germany), but according to SHENEFELT (1969-1978 [\# 86]) Germany is doubtful (AT).

997 Diospilus tuberculatus ABDINBEKOVA, 1969 [\# 81]

998 Dyscoletes lancifer (HALIDAY, 1836) [ZSM] (= similis Szépligeti)

999 Helcon angustator NEES, 1812 [\# 03] (- cylindricus Wesmael; distensor Thunberg; lignator Lepeletier \& Serville; redactor Thunberg)

1000 Helcon claviventris WESMAEL, 1835 [ZSM]

1001 Helcon nunciator (FABRICIUS, 1793) [\# 03] ( = femoralis Thomson; pedalis Cresson)

1002 Helcon tardator NEES, 1812 [ZSM]

1003 Helconidea dentator (FABRICIUS, 1804) [ZSM] (= aequator Nees; armator Marshall; rugator Ratzeburg)

1004 Helconidea mispator (LINNÉ, 1758) [ZSM] (= dentator Nees)

1005 Taphaeus biator (THUNBERG, 1822) [ZMHUB] (= speculator Haliday)

1006 Taphatus rufocephalus (TELENGA, 1950) [ZMHUB]

1007 Wrougbtonia spinator (LEPELETIER \& SERVILLE, 1827) [\# 03] (= annulicornis Nees)

\section{Histeromerinae}

1008 Histeromerus mystacinus WESMAEL, 1838 [DEI]

\section{Homolobinae}

1009 Homolobus annulicomis (NEES, 1834) [ZSM] (= testaceator auct.; simplex Herrich-Schäffer)

1010 Homolobus bohemani (BENGTSSON, 1918) [ZSM] (= bohemanni Bengtsson misspell.)

1011 Homolobus discolor (WESMAEL, 1835) [ZSM] (= pectoralis Herrich-Schäffer)

1012 Homolobus flagitator (CURTIS, 1837) [ZSM] (= chlorophthalmus Haliday; geminator Lyle) 
1013 Homolobus infumator (LYLE, 1914) [ZSM] (= japonica Watanabe; wesmaeli Bengtsson)

1014 Flomolobus truncator (SAY, 1828) [ZSM] (= calcarator Wesmael; chlorophthalma auct.; crassicalcaratus Viereck; fuscitarsis Bengtsson; melleus Cresson; simillima Enderlein; unicolor Enderlein)

\section{Ichneutinae}

1015 Ichnentes brevis WESMAEL, 1835 [\# 86]

1016 Ichneutes lapponicus THOMSON, 1895 [ZSM]

1017 Ichneutes reunitor NEES, 1816 [\# 86] (= costatus Zetterstedt)

1018 Proterops nigripennis WESMAEL, 1835 [ZSM]

1019 Pseudichneutes levis (WESMAEL, 1835) [DEI]

\section{Macrocentrinae}

1020 Austroqele longipalpis VAN ACHTERBERG, 1993 [\# 08]

1021 Macrocentrus bicolor CURTIS, 1833 [ZSM] (= limbator Ratzeburg)

1022 Macrocentrus blandus EADY \& CLARK, 1964 [ZSM]

1023 Macrocentrus buolianae EADY \& CLARK, 1964 [\# 08]

1024 Macrocentrus cingulum BRISCHKE, 1882 [\# 08] (= gifuensis auct.; grandii Goidanich)

1025 Macrocentrus collaris (SPINOLA, 1808) [ZSM] (= affiniqades Shenefelt; affinis Hedwig; dubius Wesmael; ebeninus Nees; picipes Haliday)

1026 Macrocentrus equalis LYLE, 1914 [\# 08]

1027 Macrocentrus flawus SNELLEN VAN VOLLENHOVEN, 1880 [\# 08] (= turanicus Telenga)

1028 Macrocentrus gibber EADY \& CLARK, 1964 [ZSM]

1029 Macrocentrus infirmus (NEES, 1834) [\# 86]

1030 Macrocentrus kumakoni TOBIAS, 1976 [\# 08]

1031 Macrocentrus linearis (NEES, 1811) [ZSM] (= abdominalis Fabricius; amicroploides Viereck; gifuensis Ashmead; iridescens French; pallidator Zetterstedt; tenuis Ratzeburg)

1032 Macrocentrus marginator (NEES, 1811) [ZSM] (= aegeriae Rohwer; rugator Ratzeburg)

1033 Macrocentrus nidulator (NEES, 1834) [ZSM] (= curticaudis Telenga; longicaudis Herrich-Schäffer; procenus Costa)

1034 Macrocentrus nitidus (WESMAEL, 1835) [\#09]

1035 Macrocentrus pallipes (NEES, 1812) [\# 08]

1036 Macrocentrus resinellae (LINNÉ, 1758) [\# 08] (= flavipes Ratzeburg; interstitialis Ratzeburg; intricator Ratzeburg; obscurator Ratzeburg)

1037 Macrocentrus thoracicus (NEES, 1812) [ZSM] (= gracilipes Telenga)

1038 Macrocentrus townesi VAN ACHTERBERG \& HAESELBARTH, 1983 [ZSM]

\section{Microgastrinae}

1040 Apanteles arene NIXON, 1973 [\# 71]

1041 Apanteles atreus NIXON, 1973 [\#73]

1042 Apanteles carpatus (SAY, 1836) [DEI] (= fuscicornis Cameron; hawailensis Ashmead; igae Watanabe; sarcitorius Telenga; ultericus Telenga)

1043 Apanteles corvinus REINHARD, 1880 [ZMHUB] (= aptus Papp; exilis Reinhard; lucidus Szépligeti; rasterata Fahringer) 
1044 Apanteles dorsalis (SPINOLA, 1808) [ZSM] (= cruciatus Ratzeburg; cruentatus Rondani; lictorius Reinhard; suffolciensis Morley)

1045 Apanteles ingenuoides PAPP, 1971 [\#73]

1046 Apanteles lenea NIXON, 1976 [\# 59]

1047 Apanteles longicauderra SHENEFELT, 1972 [\# 86] (= longicauda Ratzeburg)

1048 Apanteles medianus (RATZEBURG, 1852) [\# 86]

1049 Apanteles metacarpalis (THOMSON, 1895) [ZSM] (= firmus Telenga)

1050 Apanteles myron NixON, 1973 [ZSM]

1051 Apanteles nigripes (RATZEBURG, 1844) [\# 86]

1052 Apanteles obscurus (NEES, 1834) [ZSM] (= arenarius Haliday)

1053 Apanteles ononidis MARSHALL, 1889 [\# 86]

1054 Apanteles parasitellae (BOUCHÉ, 1834) [ZMHUB] (= adjunctus Marshall; flavilabris Ratzeburg; polypori Gautier \& Bonnamour; rufilabris Ratzeburg)

1055 Apanteles prinoptus PAPP, 1984 [ZMHUB] (= metacarpalis sensu Tobias)

1056 Apanteles ruficornis (NEES, 1834) [\# 86] (= hedymeles Nixon; lateralis Reinhard)

1057 Apanteles sodalis (HALIDAY, 1834) [ZMHUB] (= ater Ratzeburg; carbonarius Ratzeburg)

1058 Apanteles tedellae NIXON, 1961 [ZSM] (= epinotiae Fischer; epinoticida Fischer)

1059 Apanteles tiro (REINHARD, 1880) [ZMHUB]

1060 Apanteles xanthostigma (HALIDAY, 1834) [ZSM] (= ochrostigma Wesmael)

1061 Cotesia abjecta (MARSHALL, 1885) [ZSM]

1062 Cotesia acuminata (REINHARD, 1880) [\# 86] (= cultrator Marshall)

1063 Cotesia affinis (NEES, 1834) [ZMHUB] (= euphorbiae Bouché; globatus Panzer; harpyae Niezabitowski; vinulae Bouché)

1064 Cotesia analis (NEES, 1834) [\# 86] (= leucaniae Wilkinson; praetextatus Haliday)

1065 Cotesia ancilla (NIXON, 1974) [ZSM]

1066 Cotesia arctica (THOMSON, 1895) [ZSM]

1067 Cotesia bignellii (MARSHALL, 1885) [\# 75] (= bignelli Marshall misspell.)

1068 Cotesia brevicornis (WESMAEL, 1837) [ZSM] (= cleoceridis Marshall)

1069 Cotesia cajae (BOUCHÉ, 1834) [ZMHUB] (= difficilis Nees; glomeratus sensu Newman)

1070 Cotesia cuprea (LYLE, 1925) [\# 75]

1071 Cotesia eulipis (NIXON, 1974) [\# 58]

1072 Cotesia fermginea (MARSHALL, 1885) [ZSM]

1073 Cotesia gades (NIXON, 1974) [\# 58]

1074 Cotesia gastropachae (BOUCHÉ, 1834) [ZMHUB]

1075 Cotesia geryonis (MARSHALL, 1885) [ZMHUB]

1076 Cotesia glabrata (TELENGA, 1955) [\#75]

1077 Cotesia glomerata (LINNÉ, 1758) [ZSM] (= crataegi Ratzeburg; heterotergis Fahringer; nawaii Ashmead; nigriventris Nees; pieridis Packard; pieridivora Riley; reconditus Nees; stellatarum Bouché)

1078 Cotesia gonopterygis (MARSHALL, 1898) [\# 75]

1079 Cotesia byphantriae (RILEY, 1887) [\# 75]

1080 Cotesia isolde (NIXON, 1974) [ZSM]

1081 Cotesia jucunda (MARSHALL, 1885) [ZSM] 
1082 Cotesiajuniperatae (BOUCHÉ, 1834) [ZMHUB] (= sessilis Geoffroy ?)

1083 Cotesia kurdjumoni (TELENGA, 1955) [\# 75]

1084 Cotesia limbata (MARSHALL, 1885) [\# 86]

1085 Cotesia lineola (CURTIS, 1830) [ZSM] (= gabrielis Gautier \& Riel; lineolata Hedwig; picipes auct.)

1086 Cotesia melanoscela (RATZEBURG, 1844) [ZSM] (= creatus Balevski; solitarius Ratzeburg)

1087 Cotesia notba (MARSHALI, 1885) [ZSM]

1088 Cotesia numen (NIXON, 1974) [\#75]

1089 Cotesia ofella (NixON, 1974) [DEI] (= perspicuus Nees?)

1090 Cotesia ordinaria (RATZEBURG, 1844) [ZMHUB] (= dendrolimi Matsumura; dendrolimusi Matsumura; reconditus Hartig)

1091 Cotesia orestes (NIXON, 1974) [\#75]

1092 Cotesia picipes (BOUCHÉ, 1834) [ZMHUB]

1093 Cotesia pieridis (BOUCHÉ, 1834) [ZMHUB]

1094 Cotesia pilicornis (THOMSON, 1895) [ZSM] (= sericeus Marshall)

1095 Cotesia plutellae (KURDJUMOV, 1912) [ZSM]

1096 Cotesia praepotens (HALIDAY, 1834) [\# 86] (= placidus Haliday)

1097 Cotesia rubecula (MARSHALL, 1885) [ZSM]

1098 Cotesia rubripes (HALIDAY, 1834) [ZMHUB] (= glomeratus Curtis; coryphe Nixon)

1099 Cotesia ruficrus (HALIDAY, 1834) [ZMHUB] (= antipoda Ashmead; manilae Ashmead; narangae Viereck; sydneyensis Cameron)

1100 Cotesia salebrosa (MARSHALL, 1885) [\# 86] (= callunae Nixon)

1101 Cotesia saltator (THUNBERG, 1822) [ZMHUB]

1102 Cotesia sericea (NEES, 1834) [ZSM] (= brachycerus Thomson; praepotens auct.)

1103 Cotesia sibyllarum (WILKINSON, 1936) [ZMHUB]

1104 Cotesia specularis (SZÉpLIGETI, 1896) [ZSM] (= balcanicus Balevski)

1105 Cotesia spuria (WESMAEL, 1837) [ZSM] (= insidens Ratzeburg)

1106 Cotesia telengai (TOBIAS, 1972) [\# 74] (= amabilis Nixon)

1107 Cotesia tenebrosa (WESMAEL, 1837) [ZMHUB]

1108 Cotesia tetrica (REINHARD, 1880) [ZMHUB]

1109 Cotesia tibialis (CURTIS, 1830) [ZSM] (= aranearum Walckenaer; claustratus Gautier \& Bonnamour; congestus Nees; globatus Bouché; gracilipes Thomson; intricatus Haliday; mamestrae Matsumura; perspicuus Wesmael)

1110 Cotesia vanessae (REINHARD, 1880) [ZMHUB]

1111 Cotesia vestalis (HALIDAY, 1834) [ZMHUB] (= melitaearum Wilkinson; melittearum Wilkinson misspell.)

1112 Cotesia villana (RENHARD, 1880) [ZMHUB] ( $=$ fasciatae Gautier \& Dresnay)

1113 Cotesia vygaenarum (MARSHALL, 1885) [ZSM]

1114 Deuterixys carbonaria (WESMAEL, 1837) [ZSM] (= anomalus Lyle; gracilis Marshall)

1115 Diologaster abdominalis (NEES, 1834) [DE] (= deprimator Wesmael)

1116 Diolcogaster alvearia (FABRICIUS, 1798) [ZSM] (= alveator Thunberg; aphidum Panzer; areolatus Szépligeti)

1117 Diolcogaster connexa (NEES, 1834) [ZSM] (= consularis Haliday; dilutus Ratzeburg) 
1118 Diolcogasterflavipes (HALIDAY, 1834) [ZMHUB]

1119 Diolcogaster binqi (NIXON, 1965) [ZSM]

1120 Diologaster minuta (REINHARD, 1880) [ZMHUB]

1121 Diolcogaster scotica (MARSHALL, 1885) [ZSM] (= marginata sensu Telenga)

1122 Dolichogenidea adjuncta (NEES, 1834) [ZMHUB]

1123 Dolichogenidea albipennis (NEES, 1834) [ZMHUB]

1124 Dolichogenidea annularis (HALIDAY, 1834) [ZMHUB]

1125 Dolichogenidea appellator (TELENGA, 1949) [SCHNEE] (= litae Nixon; salverdensis Hedquist)

1126 Dolichogenidea artissima (PAPP, 1971) [\# 56] (= abila Nixon)

1127 Dolichogenidea breviventris (RATZEBURG, 1848) [\# 86] (= mesoxanthus Ruschka; nilae Telenga)

1128 Dolicbogenidea candidata (HALIDAY, 1834) [ZSM] (= cerebrator Ratzeburg misspell.; coniferae Haliday; longicauda Wesmael; terebrator Ratzeburg)

1129 Dolichogenidea credne (NIXON, 1973) [\# 57]

1130 Dolichogenidea decora (HALIDAY, 1834) [ZMHUB] (= lineatus Reinhard; sibiricus Fahringer)

1131 Dolichogenidea dilecta (HALIDAY, 1834) [ZMHUB] (= femoralis Bouché)

1132 Dolichogenidea drusilla (NrXON, 1972) [\# 56]

1133 Dolichogenidea emarginata (NEES, 1834) [ZMHUB] ( $=$ scapularis Bouché)

1134 Dolichogenidea ensiformis (RATZEBURG, 1844) [\# 67]

1135 Dolichogenidea erasmi (NIXON, 1972) [ZSM]

1136 Dolichogenidea evonymellae (BOUCHÉ, 1834) [ZMHUB] (= iarbas Nixon)

1137 Dolichogenidea gagates (NEES, 1834) [ZMHUB]

1138 Dolichogenidea gracilariae (WILKINSON, 1940) [ZSM]

1139 Dolichogenidea belleni (NIXON, 1972) [\# 66]

1140 Dolichogenidea immissa (PAPP, 1977) [\# 66]

1141 Dolichogenidea imperator (WILKINSON, 1939) [ZMHUB]

1142 Dolichogenidea impura (NEES, 1834) [\# 86]

1143 Dolichogenidea infima (HALIDAY, 1834) [ZSM]

1144 Dolichogenidea lactea (NEES, 1834) [ZMHUB]

1145 Dolichogenidea lacteicolor (VIERECK, 1911) [ZSM] (= conspersae Fiske; vinimetorum Lyle)

1146 Dolichogenidea lacteipennis (CURTIS, 1830) [\# 86]

1147 Dolichogenidea laevigata (RATZEBURG, 1848) [ZMHUB] (= calcaratus Ivanov; hoplites Ratzeburg)

1148 Dolichogenidea laevigatoides (NIXON, 1972) [ZSM]

1149 Dolichogenidea laevissimus (RATZEBURG, 1848) [\# 73]

1150 Dolichogenidea lineipes (WESMAEL, 1837) [ZSM]

1151 Dolichogenidea longipalpis (REINHARD, 1880) [ZMHUB] (= tadzhicus Telenga)

1152 Dolicbogenidea oeblkei (PAPP, 1982) [\# 84]

1153 Dolichogenidea petrovae (WALLEY, 1937) [ZSM] (= dioryctriae Wilkinson; magnus Telenga; murinanae Capek \& Zwölfer)

1154 Dolichogenidea phaloniae (WILKINSON, 1940) [ZSM]

1155 Dolicbogenidea punctiger (WESMAEL, 1837) [ZMHUB] (= itea Nixon)

1156 Dolichogenidea sicaria (MARSHALL, 1885) [ZSM] (= chrysostictus Marshall; crudelis Papp)

1157 Dolicbogenidea ultor (REINHARD, 1880) [ZMHUB] (= lactipennis Ratzeburg?) 
1158 Glyptapanteles acasta (NiXON, 1973) [DED]

1159 Glyptapanteles aliphera (NrXON, 1973) [DED] (= aliphaera Nixon misspell.)

1160 Glyptapanteles antinoe (NrXON, 1973) [DEI (= antione Nixon misspell.)

1161 Glyptapanteles compressiventris (MUESEBECK, 1921) [ZMHUB]

1162 Glyptapanteles fausta (NIXON, 1973) [ZSM]

1163 Glptapanteles fraternus (REINHARD, 1880) [ZMHUB]

1164 Gyptapanteles fulvipes (HALIDAX, 1834) [ZMHUB] (= glomeratus sensu Nees)

1165 Glyptapanteles inclusus (RATZEBURG, 1844) [ZMHUB] (= rectinervis Telenga)

1166 Glyptapanteles lateralis (HALIDAY, 1834) [ZMHUB]

1167 Glyptapanteles liparidis (BOUCHÉ, 1834) [ZMHUB] (= awanomeigae Watanabe; fulvipes Howard \& Fiske; japonicus Ashmead; nemorum Hartig; politus Ashmead; posticae Sonan)

1168 Glyptapanteles luciana (NIXON, 1973) [\#32] Remark: CvA considers the species as belonging to the genus Protapanteles.

1169 Glyptapanteles mygdonia (NIXON, 1973) [ZSM]

1170 Glyptapanteles octonarius (RATZEBURG, 1852) [ZMHUB] (= lucifugus Lyle; stauropodis Bridgman)

1171 Glyptapanteles pallipes (REINHARD, 1880) [ZMHUB] (= pallidipes Marshall; reinhardi Wilkinson)

1172 Glyptapanteles porthetriae (MUESEBECK, 1928) [ZSM]

1173 Glyptapanteles ripus (PAPP, 1983) [DEM]

1174 Glyptapanteles rubens (REINHARD, 1880) [ZMHUB]

1175 Glyptapanteles sibiricus (PAPP, 1983) [DEI] Remark: In the DEI collection there are some specimens ex Lymantria dispar, det. PAPP 1995.

1176 Glyptapanteles vitripennis (CURTIS, 1830) [ZMHUB] (= fulcriger Wesmael; impavidus Gautier \& Dresnay)

1177 Hygroplitis rugulosus (NEES, 1834) [ZMHUB] (= deprimator Curtis; infumatus Haliday; opacus Ruthe)

1178 Hygroplitis russatus (HALIDAY, 1834) [ZMHUB] (= aomoriensis Matsumura; basalis Stephens; dimidiatus Wesmael)

1179 Iconella aeolus (NIXON, 1965) [\# 69]

1180 Iconella lacteoides (NIXON, 1965) [\# 69] (= memorabilis Alexeev)

1181 Iconella merula (REINHARD, 1880) [ZMHUB]

1182 Illidops butalidis (MARSHALL, 1889) [\# 68]

1183 Illidops suemus (REINHARD, 1880) [ZMHUB] (= dion Nixon; polonicus Fahringer; sesostris Nixon)

1184 Microgaster alebion NIXON, 1968 [ZSM]

1185 Microgaster areolaris THOMSON, 1895 [ZSM] (= striatoscutellaris Zilahi-Kiss)

1186 Microgaster auriculata (FABRICIUS, 1804) [ZMHUB]

1187 Microgaster australis THOMSON, 1895 [\# 86] (= deprimator auct.)

1188 Microgaster crassicomis RUTHE, 1860 [ZMHUB]

1189 Microgaster dudichi PAPP, 1961 [DED]

1190 Microgaster elegans HERRICH-SCHÄFFER, 1838 [\# 86]

1191 Microgaster erro NIXON, 1968 [\# 65] 
1192

1193

1194

1195

1196

1197

1198

1199 Microgaster nigricans NEES, 1834 [ZMHUB]

1200 Microgaster nitidula WESMAEL, 1837 [\# 86]

1201 Microgaster nobilis REINHARD, 1880 [ZMHUB]

1202 Microgaster novicia MARSHALL, 1885 [ZSM] (= swammerdamiae Muesebeck)

1203 Microgasterparvistriga THOMSON, 1895 [\# 55]

1204 Microgaster polita MARSHALL, 1885 [ZSM]

1205 Microgaster postica NEES, 1834 [ZMHUB] (= marginellus Wesmael)

1206 Microgaster procera RUTHE, 1860 [ZSM] (= intermedia Ivanov)

1207 Microgaster ruficoxis RUTHE, 1858 [\# 86]

1208 Microgaster stictica RUTHE, 1858 [ZSM] (= confusa Papp)

1209 Microgaster subcompleta NEES, 1834 [ZMHUB] (= annulipes Curtis; carinata Packard)

1210 Microgaster subtilipunctata PAPP, 1959 [\# 55] (= obsepiens Nixon)

1211 Microplitis aduncus (RUTHE, 1860) [ZSM] (= brachycera Thomson)

1212 Microplitis capeki NixON, 1970 [\# 72]

1213 Microplitis cebes NIXON, 1970 [ZSM]

1214 Microplitis combinatus (PAPP, 1984) [\# 72]

1215 Microplitis decens TOBIAS, 1964 [ZSM]

1216 Microplitis decipiens PRELL, 1925 [ZMHUB]

1217 Microplitis deprimator (FABRICIUS, 1798) [DEI] (= ingratus Haliday; sordipes Nees; tau Ratzeburg)

1218 Microplitis docilis NIXON, 1970 [ZMHUB]

1219 Microplitis eremita REINHARD, 1880 [DE]

1220 Microplitis erythrogaster ABDINBEKOVA, 1969 [\# 72]

1221 Microplitis flavipalpis (BRULLÉ, 1832) [ZSM] (= ruricola Lyle)

1222 Microplitis fordi NIXON, 1970 [ZSM]

1223 Microplitis fulwicornis (WESMAEL, 1837) [ZSM] (= calcarata sensu Nixon)

1224 Microplitis beterocerus (RUTHE, 1860) [BMNH]

1225 Microplitis idia NIXON, 1970 [\# 72]

1226 Microplitis impressus (WESMAEL, 1837) [\# 72] (= sispes Nixon)

1227 Microplitis lugubris (RUTHE, 1860) [ZSM] (= borealis Marshall; coracina Thomson; rutheana Fahringer)

1228 Microplitis mandibularis (THOMSON, 1895) [ZSM] (= pallidicornis Marshall) 
1229 Microplitis mediator (HALIDAY, 1834) [DED (= dorsalis Nees; halidayi Fahringer; mediana Ruthe; pseudomediana Fahringer)

1230 Microplitis moestus (RATZEBURG, 1852) [\# 72]

1231 Microplitis ocellatae (BOUCHÉ, 1834) [DEI] (= canaliculatus Wesmael)

1232 Microplitis pellucidus TELENGA, 1955 [\# 72]

1233 Microplitis ratzeburgii (RUTHE, 1858) [ZSM] (= cerurae Matsumura; ratzeburgi Ruthe; spinolae Ratzeburg)

1234 Microplitis semicircularis (RATZEBURG, 1844) [\# 86]

1235 Microplitis sofron NIXON, 1970 [ZSM] (= stigmaticus auct.)

1236 Microplitis spectabilis (HALIDAY, 1834) [ZSM] (= fossulatus Bouché; parvulus Ruthe; seuratii Marshall; testaceipes Cameron)

1237 Microplitis spinolae (NEES, 1834) [ZMHUB] (= radiorimata Telenga)

1238 Microplitis strenuus REINHARD, 1880 [DEI] (= gracilis Ruthe)

1239 Microplitis tristis (NEES, 1834) [DEI] (= dolens Marshall)

1240 Microplitis tuberulatus (BOUCHÉ, 1834) [ZMHUB] (= fumipennis Ratzeburg)

1241 Microplitis tuberculifer (WESMAEL, 1837) DEI] (= calcarata Thomson; manevali Gautier \& Bonnamour; trochanterata Thomson; tuberculifera Wesmael misspell.)

1242 Microplitis varipes (RUTHE, 1860) [DEI (= stigmaticus Ratzeburg ?; stigmativetus Shenefelt?)

1243 Microplitis viduus (RUTHE, 1860) [ZSM]

1244 Microplitis xanthopus (RUTHE, 1860) DEI] (= tenuipes Thomson)

1245 Napamus vipio (REINHARD, 1880) [ZMHUB]

1246 Paroplitis wesmali (RUTHE, 1860) [ZMHUB]

1247 Pboletesor arisba (NIXON, 1973) [\# 71]

1248 Pholetesor bicolor (NEES, 1834) [\# 30] (= exiguus Haliday; pedias Nixon; pedius Nixon. misspell.; umbellatarum Haliday)

1249 Pboletesor circumscriptus (NEES, 1834) [\# 86] (= ardeaepenellae Bouché; blancardellae Bouché; flavolimbatus Ratzeburg; lautellus Marshall; lividipes Wesmael; longicauda Fahringer)

1250 Pholetesor elpis (NrXON, 1973) [ZSM] (= girkanus Tobias?)

1251 Pholetesor laetus (MARSHALL, 1885) [\# 86] (= metallicus Jakimavicius)

1252 Pholetesor maritimus (WILKINSON, 1941) [ZSM]

1253 Pholetesor nanus (REINHARD, 1880) [ZMHUB] (= szoecsi Papp)

1254 Pholetesor phaetusa (NIXON, 1973) [ZSM]

1255 Pholetesor viminetorum (WESMAEL, 1837) [ZSM] (= fuliginosus Wesmael)

1256 Protapanteles anchisiades (NIXON, 1973) [ZSM]

1257 Protapanteles andromica (NIXON, 1976) [ZSM]

1258 Protapanteles delitutus (PAPP, 1984) [\# 73]

1259 Protapanteles enepbes (NIXON, 1965) [ZSM]

1260 Protapanteles formosus (WESMAEL, 1837) [\# 86] (= marshalli Bignell) Remark: PAPp (1988 [\#94]) treated the species in the Genus Distatrix MASON.

1261 Protapanteles iapetus (NIXON, 1976) [ZSM]

1262 Protapanteles immunis (HALIDAY, 1834) [ZSM] 
1263 Protapanteles incertus (RUTHE, 1859) [\# 59] (= caberae Marshall; jugosus Lyle; mihalyiu Papp)

1264 Protapanteles majalis (WESMAEL, 1837) [ZSM] (= callidus auct)

1265 Protapanteles mandanis (NIXON, 1965) [\# 59]

1266 Protapanteles parallelus (LYLE, 1917) [\# 73] (= lylei Shenefelt)

1267 Protapanteles popularis (HALIDAY, 1834) [\# 86]

1268 Protapanteles triangulator (WESMAEL, 1837) [ZSM]

1269 Rasivalva calceata (HALIDAY, 1834) [\# 86] (= pubescens Ratzeburg)

1270 Rasivalua marginata (NEES, 1834) [ZMHUB]

1271 Satbon falcatus (NEES, 1834) [ZMHUB] (= equestris Haliday; gladiator Szépligeti; minor Fahringer; priapus Gautier \& Cleu)

\section{Miracinae}

1272 Mirax rufliabris HALIDAY, 1833 [ZMHUB] (= dryochares Marshall; nanivorae Fischer; spartii Haliday)

\section{Opiinae}

1273 Ademon decrescens (NEES, 1811) [ZSM] (= mutuator Nees) Remark: Bracon mutuator NEES, 1811 is a hitherto unpublished synonym $(\mathrm{CvA})$.

1274 Aulonotus comatus (WESMAEL, 1835) [ZSM] (= sulcifera Papp) Remark: CvA considers the species as belonging to the genus Xynobius.

1275 Biosteres analis (WESMAEL, 1835) [DEI] (= colorativentris Fischer) Remark: The types of analis belong to Biosteres and are conspecific with colorativentris (CvA). FISCHER, PAPP and TOBIAS treated the species in Opius.

1276 Biosteres arenarius (STELFOX, 1959) [ZSM]

1277 Biosteres bicolor (WESMAEL, 1835) [ZSM] (= vagator Förster)

1278 Biosteres blandus (HALIDAY, 1837) [ZSM] Remark: CvA considers the species as belonging to the genus Chilatricbic.

1279 Biosteres borealis (ZETTERSTEDT, 1838) [\#15] (= brevipalpis auct.)

1280 Biosteres bremeri (BENGTSSON, 1926) [\# 14]

1281 Biosteres brevisulcus (THOMSON, 1895) [ZSM] (= melanosoma Szépligeti)

1282 Biosteres carbonarius (NEES, 1834) [ZSM] (= impressus Wesmael; procerus Wesmael)

1283 Biosteres caudatulus (THOMSON, 1895) [ZSM]

1284 Biosteres baemorrboews (HALIDAY, 1837) [ZSM] (= castaneiventris Thomson; palaearcticus Szépligeti)

1285 Biosteres longicauda (THOMSON, 1895) [ZSM]

1286 Biosteres micans (STELFOX, 1957) [ZSM] ( = nitidus Stelfox)

1287 Biosteres placidus (HALIDAY, 1837) [ZSM] (= indotatus Viereck; melanocerus Wesmael; tarsator Thomson)

1288 Biosteres punctiscuta (THOMSON, 1895) [ZSM]

1289 Biosteres rusticus (HALIDAY, 1837) [\# 14]

1290 Biosteres scabriculus (WESMAEL, 1835) [\# 14]

1291 Biosteres spinaciae (THOMSON, 1895) [ZSM] (- fissus Jakimavicius; hyosciamellus Viereck; pegomyiae Gahan) 
1292 Biosteres syluaticus (HAIIDAY, 1837) [\# 14] (= clypealis Thomson; nitida Szépligeti) Remark: Opius clypealis THOMSON, 1895 is a hitherto unpublished synonym (CvA).

1293 Biosteres wesmaelii (HALIDAY, 1837) [ZSM] (= carbonarius Wesmael; ultor Förster; wesmaeli Haliday misspell.)

1294 Cepbaloplites mocsaryi SZÉPLIGETI, 1897 [\# 14]

1295 Coleopius mgiventris (THOMSON, 1895) [\# 15] Remark: Coleopius migiventris (THOMSON, 1895) is a new combination (comb. nov., $\mathrm{CrA}$ ).

1296 Desmiostoma parvulum (WESMAEL, 1835) [ZSM] (= nudiscutum Fischer; ziratus Papp)

1297 Diachasma compressiventre (FISCHER, 1964) [\# 14]

1298 Diachasma fulgidum (HALIDAY, 1837) [ZMHUB]

1299 Diachasma striatum (FÖRSTER, 1862) [ZMHUB] Remark: CvA prefers the original combination Atoreuteus striatus FÖRSTER, 1862 for this species.

1300 Diachasma xanthopus (FÖRSTER, 1862) [ZMHUB] Remark: CvA prefers the original combination Batbystomus xantbopus FÖRSTER, 1862 for this species.

1301 Eurytenes abnormis (WESMAEL, 1835) [ZMHUB]

1302 Eurytenes macrocerus (THOMSON, 1895) [ZSM] (= hians Stelfox)

1303 Opius aethiops HALIDAY, 1837 [ZSM]

1304 Opius agromyzicola FISCHER, 1967 [ZSM]

1305 Opius amarellae FISCHER, 1967 [\# 14]

1306 Opius ambiguns WESMAEL, 1835 [ZSM]

1307 Opius apfelbeckianus FISCHER, 1967 [\# 14]

1308 Opius aureliae FISCHER, 1957 [ZSM] (= oetztalicola Fischer) Remark: CvA considers the species as belonging to the genus Opiostomus. Opius oetztalicola FISCHER, 1998 is a hitherto unpublished synonym (CrA).

1309 Opius bajulus HALIDAY, 1837 [\# 14] Remark: CvA considers the species as belonging to the genus Xynobius.

1310 Opius bicoloriformis FISCHER, 1957 [\# 14] (= bicolor Szépligeti; discolor Papp)

1311 Opius blantoni FISCHER, 1964 [\# 90] (= brusceanus Fischer; vockerothi Fischer) Remark: CvA considers the species as belonging to the genus Opiostomus.

1312 Opius brevipalpis THOMSON, 1895 [ZSM] (= mutus Fischer) Remark: Opius mutus FISCHER, 1964 is a hitherto unpublished synonym (CvA).

1313 Opius caelatus HALIDAY, 1837 [ZSM] (= isomera Förster; pallipes Förster) Remark: CvA considers the species as belonging to the genus Xynobius.

1314 Opius caesus HALIDAY, 1837 [ZSM] (= hydrelliae Rimsky-Korsakov; punctiventris Thomson; subtilis Szépligeti)

1315 Opius caricivorae FISCHER, 1964 [ZSM]

1316 Opius caudifer FISCHER, 1958 [ZSM]

1317 Opius celsus HALIDAY, 1837 [ZSM]

1318 Opius ciceris FISCHER, 1974 [\# 20]

1319 Opius cinctiventris FISCHER, 1959 [\# 20] Remark: CvA considers the species as belonging to the genus Xynobius.

1320 Opius cingulatus WESMAEL, 1835 [ZSM] (= dentifer Thomson; stramineipes Thomson)

1321 Opius circulator (NEES, 1834) [ZSM] Remark: CvA considers the species as belonging to the genus Apodesmia. 
1322 Opius compar MARSHALt, 1894 [\# 40] (= pulchrithorax Fischer)

1323 Opizs coracinus THOMSON, 1895 [ZSM] (= silvicola Szépligeti)

1324 Opius crassicrus THOMSON, 1895 [ZSM] (= nigticinctus Fischer)

1325 Opius crassipes WESMAEL, 1835 [ZSM]

1326 Opius cubitalis FISCHER, 1959 [\# 20] Remark: CvA considers the species as belonging to the genus Xynobius.

1327 Opius curvatus FISCHER, 1957 [ZSM]

1328 Opius depeculator (FörSTER, 1862) [ZSM] (= semiaciculatus Stelfox)

1329 Opiws diversus SZEPLIGETI, 1898 [ZSM] (= exiguus auct.)

1330 Opius docilis HALIDAY, 1837 [ZSM] (= parvungula Thomson) Remark: Opius parvungula THOMSON, 1895 is a hitherto unpublished synonym (CvA).

1331 Opius dureseaui FTSCHER, 1975 [ZSM]

1332 Opius exiguHs WESMAEL, 1835 [ZSM]

1333 Opius fallax SZÉpLIGETI, 1896 [\# 14]

1334 Opins fulvicollis THOMSON, 1895 [\# 15] (= cupidus Gahan) Remark: CvA considers the species as belonging to the genus Utetes.

1335 Opius fuscipennis WWESMAEL, 1835 [ZSM]

1336 Opius geniculatus THOMSON, 1895 [ZSM] (= albicoxis Marshall) Remark: CvA considers the species as belonging to the genus Xynobius.

1337 Opius gigapiceus Frscher, 1989 [\# 20]

1338 Opius gracilis FISCHER, 1957 [ZSM] (= csikii Fischer; minor Fischer; nigrithorax Fischer) Remark: Opius minor FISCHER, 1957 is a hitherto unpublished synonym (CVA).

1339 Opius beringi FISCHER, 1962 [\# 14]

1340 Opius bolconotus FISCHER, 1958 [ZSM] Remark: CVA considers the species as belonging to the genus Xynobius.

1341 Opius inmatientis FISCHER, 1957 [ZSM] Remark: CvA considers the species as belonging to the genus Opiostomus.

1342 Opius incisulus FISCHER, 1964 [\# 14] Remark: CvA considers the species as belonging to the genus Apodesmia.

1343 Opius instabilis WeSMAEL, 1835 [\# 15]

1344 Opius irregularis WESMAEL, 1835 [ZSM] (= bipustulatus Fischer) Remark: CvA considers the species as belonging to the genus Apodesmia.

1345 Opius iuxtangelum FISCHER, 1978 [ZSM]

1346 Opius karesuandensis FISCHER, 1964 [ZSM]

1347 Opius laetatorius FISCHER, 1958 [\# 20]

1348 Opius laevigatus (FÖRSTER, 1862) [\# 14]

1349 Opius leptostigma.WESMAEL, 1835 [\#15] (= percontator Fischer) Remark: Opius percontator FISCHER, 1964 is a hitherto unpublished synonym (CvA). CvA considers the species as belonging to the genus Opiostomus.

1350 Opius levis WESMAEL, 1835 [ZSM] (= filicornis 'Thomson; varipes Szépligeti) Remark: Opius filicornis THOMSON, 1895 is a hitherto unpublished synonym (CvA).

1351 Opius longicornis THOMSON, 1895 [ZSM]

1352 Opius longiradialis FISCHER, 1957 [\# 14]

1353 Opius lonicerae FISCHER, 1958 [ZSM] 
1354 Opius luidus SZÉPLIGETI, 1896 [\# 14]

1355 Opius lugens HALIDAY, 1837 [ZSM] (= abscissus Thomson; adveniens Fischer; obscurus Szépligeti)

1356 Opius maculipes WESMAEL, 1835 [ZSM] (= addendus Fischer; turcmenicus Fischer) Remark: CvA considers the species as belonging to the genus Xynobius.

1357 Opius magnicauda FISCHER, 1958 [ZSM]

1358 Opius mirabilis FTSCHER, 1958 [ZSM]

1359 Opius monticola SZÉPLIGETI, 1898 [\# 20]

1360 Opius mundus FÖRSTER, 1862 [ZSM]

1361 Opius neopendulus FISCHER, 1989 [\# 20]

1362 Opius nigricolor FISCHER, 1960 [\# 14]

1363 Opius nigricoloratus FISCHER, 1958 [ZSM]

1364 Opius nitidulator (NEES, 1834) [\# 14] (= vittatus Ruschka)

1365 Opius ocellatus WESMAEL, 1835 [ZSM] (= areolaris Thomson; brutus Papp; hungaricus Szépligeti) Remark: CvA considers the species as belonging to the genus Apodesmia.

1366 Opius ocbrogaster WESMAEL, 1835 [ZSM] (= neopusillus Fischer; nigriceps Szépligeti)

1367 Opius ocbropus THOMSON, 1895 [\# 40] Remark: CvA considers the species as belonging to the genus Utetes.

1368 Opius ocreatus PAPP, 1979 [\# 70] Remark: CvA considers the species as belonging to the genus Opiostomus.

1369 Opius orbiculator (NEES, 1811) [\# 20] (= breviscapus Thomson) Remark: Opius breviscapus THOMSON, 1895 is a hitherto unpublished synonym (CvA).

1370 Opius pactus HALIDAY, 1837 [ZSM]

1371 Opius pallipes WeSMAEL, 1835 [ZSM] (= adaequator Förster; exilis Haliday; lividipes Förster; pallidipes Marshall; piceus Thomson; subsulcata Förster) Remark: Opius piceus THOMSON, 1895 is a hitherto unpublished synonym (CvA).

1372 Opius pendulus HALIDAY, 1837 [ZSM] (= latipes Fischer) Remark: Opius latipes FISCHER, 1958 is a hitherto unpublished synonym (CvA).

1373 Opius pircbitticola FISCHER, 1974 [ZSM]

1374 Opius polyzonius WESMAEL, 1835 [ZSM] Remark: CrA considers the species as belonging to the genus Xynobius.

1375 Opius propodealis FISCHER, 1958 [ZSM]

1376 Opius pulcherrimus FISCHER, 1958 [ZSM]

1377 Opius pulchriceps SzÉPLIGETI, 1898 [\# 14] (= ilicis Nixon; pulchriventris Fischer; vexator Fischer) Remark: Opius ilicis NIXON, 1939, Opius pulchriventris FISCHER, 1958, and Opius vexator FISCHER, 1964 are hitherto unpublished synonyms (CVA).

1378 Opius pulicariae FISCHER, 1969 [ZSM]

1379 Opius pygmaeator (NEES, 1811) [ZSM] (= funebris Wesmael; meracus Fischer; pygmeator Nees misspell.) Remark: Opius funebris WESMAEL, 1835 and Opius meracus FISCHER, 1960 are hitherto unpublished synonyms (CvA).

1380 Opius pygmaeus FISCHER, 1962 [\# 20]

1381 Opius radialis FISCHER, 1957 [ZSM]

1382 Opius reconditor WESMAEL, 1835 [\# 15]

1383 Opius rex FISCHER, 1958 [ZSM]

1384 Opius rudis WESMAEL, 1835 [ZSM] (= carinaticeps Gahan) 
1385 Opius rufipes WESMAEL, 1835 [ZSM] (= taeniata Förster) Remark: Apodesmia taeniata FöRSTER, 1860 is a hitherto unpublished synonym (CvA). CVA considers the species as belonging to the genus Apodesmia.

1386 Opius saevulus FISCHER, 1958 [ZSM] Remark: CvA considers the species as belonging to the genus Apodesmia.

1387 Opius saevus HALIDAY, 1837 [ZSM] Remark: CvA considers the species as belonging to the genus Apodesmia.

1388 Opius sigmodus PAPP, 1981 [ZSM]

1389 Opius similis SZÉPLIGETI, 1898 [ZSM] (= altimontanus Fischer; basirufus Fischer; nodatus Fischer; periclymenii Fischer; similiformis Fischer; xylostei Marshall) Remark: CvA considers the species as belonging to the genus Apodesmia. Opius altimontanus FISCHER, 1969, Opius nodatus FISCHER 1958, Opius peridymenit FISCHER, 1964 and Opius similiformis FISCHER, 1957 are hitherto unpublished synonyms (CvA).

1390 Opius similoides FISCHER, 1962 [\#20] Remark: CvA considers the species as belonging to the genus Apodermia.

1391 Opius singularis WESMAEL, 1835 [ZSM] (= arenosus Szépligeti; clarus Haliday; spretus Haliday)

1392 Opius soenderupianus FISCHER, 1967 [\# 14]

1393 Opius staryi FISCHER, 1958 [ZSM]

1395 Opius tenellae FISCHER, 1969 [ZSM]

1396 Opius tersus (FÖRSTER, 1862) [ZSM] (= consors Fischer; minimus Fischer)

1397 Opius tirolensis FISCHER, 1958 [\# 14]

1398 Opius tuberculifer FISCHER, 1958 [\# 14]

1399 Opius turcicus FISCHER, 1960 [ZSM]

1400 Opius variegatus SZÉPLIGETI, 1896 [ZSM]

1401 Opius victus HALIDAY, 1837 [\# 20] Remark: CvA considers the species as belonging to the genus Atormus.

1402 Opius woerziphagus FISCHER, 1967 [\# 14]

1403 Psyttalia carinata (THOMSON, 1895) [DEI] (= rhagoleticolus Sachtleben) Remark: Opius rhagoleticolus SACHTLEBEN, 1934 is an unpublished synonym of carinata (CVA \& VAN ZUIJLEN in prep.).

1404 Rhinoplus laevigatus FÖRSTER, 1862 [ZMHUB]

1405 Stemaulopius beieri FISCHER, 1968 [ZMHUB]

1406 Utetes aemulus (HALIDAY, 1837) [ZSM] (= longipes Fischer; melbus Papp) Remark: Opius longipes FISCHER, 1957 is a hitherto unpublished synonym (CVA).

1407 Utetes caudatus (WESMAEL, 1835) [ZSM] (= excerta Thomson misspell.; exsertus Thomson)

1408 Utetes magnus (FISCHER, 1958) [\# 20] (= scrutator Tobias) Remark: Opius scrutator TOBIAS, 1977 is a hitherto unpublished synonym $(\mathrm{CvA})$.

1409 Utetes posticatae (FISCHER, 1957) [\# 84] (= hilaris Fischer; hostium Fischer; seebensteinensis Fischer) Remark: Opius bilaris FISCHER, 1962, Opius bostium FISCHER, 1964, and Opius seebensteinensis FISCHER, 1959 are hitherto unpublished synonyms (CVA).

1410 Utetes rotundiventris (THOMSON, 1895) [ZSM]

1411 Utetes ruficeps (WESMAEL, 1835) [ZSM]

1412 Utetes testaceus (WESMAEL, 1838) [\# 15] 
1413 Utetes trisulcus (THOMSON, 1895) [\# 20]

1414 Utetes truncatus (WESMAEL, 1835) [\# 15]

1415 Utetes zelotes (MARSHALL, 1891) [ZSM] (= exsertus Fischer; incertus Fischer misspell.; insertus Fischer)

\section{Orgilinae}

1416 Microtypus trigonus (NEES, 1834) [\# 12] (= ilinskyi Glowacki \& Karpinski)

1417 Microtypus nesmaelii RATZEBURG, 1848 [\# 12] (= dioryctriae Rohwer)

1418 Orgilus abbreviator (RATZEBURG, 1852) [\# 88] (= nanellae Tobias)

1419 Orgilus acbterbergi TAEGER, 1989 [\# 89]

1420 Orgilus anurus THOMSON, 1895 [DED] (= scaber Muesebeck)

1421 Orgilus capeki TAEGER, 1989 [\# 89]

1422 Orgilus dovnariTOBIAS, 1986 [\# 89] (= ukrainicus Tobias)

1423 Orgilus grunini TOBIAS, 1986 [DEI]

1424 Orgilus interjectus TAEGER, 1989 [DET]

1425 Orgilus ischnus MARSHALL, 1898 [DE] (= subtilirugosus Papp)

1426 Orgilus leptocephalus (HARTIG, 1838) [\# 89] (= hyperboreus Hellén; obscurator auct.; rugulosa Fahringer)

1427 Orgilus minor'TAEGER, 1989 [DED]

1428 Orgilus moldavicus TOBIAS, 1986 [\# 89]

1429 Orgilus obscurator (NEES, 1812) [ZMHUB]

1430 Orgilus parvipenis THOMSON, 1895 [DED (= curtipennis Fischer; decoratus Hellén; discolor Hellén; macroptera Rudow; micropterus Morley)

1431 Orgilus patzaki TAEGER, 1989 [DED]

1432 Orgilus perplexus TAEGER, 1989 [DEI]

1433 Orgilus pimpinellae NIEZABITOWSKI, 1910 [DED]

1434 Orgilus punctulator (NEES, 1812) [\# 89]

1435 Orgilus radialis] AKIMAVICIUS, 1972 [\# 89]

1436 Orgilus rubrator (RATZEBURG, 1852) [\# 89] (= nordmani Hellén)

1437 Orgilus rugosus (NEES, 1834) [DED]

1438 Orgilus saponariellae TAEGER, 1989 [\# 89]

1439 Orgilus simillimus TAEGER, 1989 [\# 89]

1440 Orgilus sticticus TAEGER, 1989 [\# 89]

1441 Orgilus temporalis TOBIAS, 1976 [\# 89] (= rufina Fahringer)

1442 Orgilus tobiasi TAEGER, 1989 [\# 89]

\section{Rhyssalinae}

1443 Dolopsidea indagator (HALIDAY, 1836) [ZSM] (= aculeator Marshall; caucasica Tobias; hastifer Marshall; rhodopeus Zaykov; testacea Fahringer)

1444 Oncophanes minutus (WESMAEL, 1838) [DEI] (= laevigatus Ratzeburg; lanceolator Nees)

1445 Pseudobatbystomus funestus (HALIDAY, 1836) [DET]

1446 Rbyssalus clavator HALIDAY, 1833 [DED (= tuberculatus Wesmael) 


\section{Rogadinae}

1447 Aleiodes affinis (HERRICH-SCHÄFFER, 1838) [\# 86]

1448 Aleiodes albitibia (HERRICH-SCHÄFFER, 1838) [ZMHUB] (= heterogaster Wesmael)

1449 Aleiodes alternator (NEES, 1834) [\# 86]

1450 Aleiodes apiculatus (FAHRINGER, 1932) [ZMHUB] (= apicalis Reinhard; negativus Tobias)

1451 Alioides articus (THOMSON, 1892) [DEI]

1452 Aleiodes assimilis (NEES, 1811) [\# 86] Remark: The species is not a synonym of Aleiodes bicolor (result of rearing) ( $\mathrm{CVA}$ ).

1453 Aleiodes aterrimus (RATZEBURG, 1852) [DED] (Egrandis Giraud; malaisei Shestakov) Remark: Aleiodes grandis GIRAUD, 1857 is a hitherto unpublished synonym (CvA).

1454 Aleiodes bicolor (SPINOLA, 1808) DEX] (= basalis Costa; incertoides Telenga; incertus Kokujev; latus Telenga; maculatus Papp; pallidus Hellén; rufiventris Fahringer; tener Kokujev; zygaenae Nees) Remark: See remark of Aleiodes assimilis (CvA).

1455 Aleiodes borealis (THOMSON, 1892) [ZSM] (= vitripennis Telenga)

1456 Aleiodes circumscriptus (NEES, 1834) [DED (= bistrigatus Roman; nigriceps Wesmael; pictus Herrich-Schäffer; testaceus sensu Spinola) Remark: If A. pictus (HERRICH-SCHÄFFER, 1838 ) is considered to be a valid species ( $\mathrm{CvA}$ ) it is present in Germany, too (ZSM).

1457 Aleiodes coxalis (SPINOLA, 1808) [DE] (= kolthoffi Fahringer; tristis Wesmael)

1458 Aleiodes cruentus (NEES, 1834) [DED] (= basalis Hellén; dorsalis Herrich-Schäffer; nigricans Fahringer; nigromaculata Hellén; rufofasciata Hellén)

1459 Aleiodes dispar (HALIDAY, 1833) [DET]

1460 Aleiodes dissector (NEES, 1834) [DE] (= diversus Szépligeti)

1461 Aleiodes ductor (THUNBERG, 1822) [DED] (= reticulator Nees; similis Szépligeti)

1462 Aleiodes esenbeckii (HARTIG, 1838) [\# 86]

1463 Aleiodes eurinus (TELENGA, 1941) [DET] (= nigratus Papp; nigripes Papp)

1464 Aleiodes laevigatus (HERRICH-SCHÄFFER, 1838) [\# 86]

1465 Aleiodes lanceolatus (HERRICH-SCHÄFFER, 1838) [\# 86]

1466 Aleiodes linearis HELLÉN, 1927 [ZSM]

1467 Aleiodes miniatus (HERRICH-SCHÄFFER, 1838) [DET] (= bicolotatus Boheman; formosus Giraud)

1468 Aleiodes modestus (REINHARD, 1863) [ZMHUB] (= piceus Fahringer)

1469 Aleiodes morio (REINHARD, 1863) [\# 86]

1470 Aleiodes nigriconis WESMAEL, 1838 [ZSM]

1471 Aleiodes nobilis (HALIDAY, 1834) [DET] (= medianus Thomson)

1472 Aleiodes pallidator(THUNBERG, 1822) [DEI] ( $=$ unicolor Wesmael)

1473 Aleiodes pallidicomis (HERRICH-SCHÄFFER, 1838) [ZMHUB] (= coloratus Hellén; hirtus Thomson; pallidipennis Dalla Torre)

1474 Aleiodes picipes (HERRICH-SCHÄFFER, 1838) [\# 86]

1475 Aleiodes prator (REINHARD, 1863) [ZMHUB]

1476 Aleiodes pulchripes WESMAEL, 1838 [\# 86] (= pulchricornis Kolubajev)

1477 Aleiodes ruficonis (HERRICH-SCHÄFFER, 1838) [DEI] (= brevicornis Wesmael; dimidiatus auct.; gasterator auct.; infuscatus Hellén; nigripalpis Wesmael; nigripes Hellén; nigrobasalis Hellén; ruficollis Hellén)

1478 Aleiodes rugulosus (NEES, 1811) [DET] (= pictus Kokujev) 
1479 Aleiodes seriatus (HERRICH-SCHÄFFER, 1838) [DEI (= kuslitzkyi Tobias; vittiger Wesmael)

1480 Aleiodes signatus (NEES, 1811) [DED] (= annulipes Herrich-Schäffer; balteatus Curtis; dubius Telenga; geniculator Nees; nigerrimus Fahringer; rufus Papp)

1481 Aleiodes similis (CURTIS, 1834) [\# 86] (= ochraceus Curtis; pallidus Bouché; spathuliformis Haliday; subucola Curtis)

1482 Aleiodes mipunctator (THUNBERG, 1822) [DE] (= irregularis Wesmael; nigrescens Hellén)

1483 Aleiodes varius (HERRICH-SCHÄFFER, 1838) [\# 35]

1484 Petalodes compressor (HERRICH-SCHÄFFER, 1838) [DEI] (= unicolor Wesmael) Remark: The species is likely to be a derived Aleiodes species (CvA).

1485 Rogas luteus NEES, 1834 [DED] (= testaceus Fabricius)

1486 Triraphis tricolor (WESMAEL, 1838) [DEI (= rimulosa Marshall)

\section{Sigalphinae}

1487 Acampsis alternipes (NEES, 1816) [ZSM]

1488 Sigalphus irrorator (FABRICIUS, 1775) [ZMHUB]

\section{List of species with uncertain Records from Germany}

Perilitus benacensis HAESELBARTH, 1999 (see HAESELBARTH 1999 [\# 34])

Opius subaffinis FISCHER, 1962: The record from Germany most likely based on a doubtful specimen in the ZSM, labelled by FISCHER as "subaffinis?".

Microchelonus foersteri TOBIAS, 1999

\section{Microchelonus nigellus TOBIAS, 1999}

Microcbelonus scaberrimus TOBIAS, 1999: All three species described from the FÖRSTER collection, but it remains uncertain, if the material is from Germany or not, as all species are based on material without any given locality.

Hormius similis SzÉPLIGETI, 1896: SB has seen material from Germany, but the reference can not now be found.

Kerorgilus zonator (SZÉPLIGETI, 1896): The German record is obviously only based on SzÉPLIGETI's remark "...ein Exemplar, welches noch von Förster als Org. zonator bezeichnet wurde...". This does not mean that the specimen is from Germany. The occurence of this distinct species in Germany is unlikely. (AT).

Aleiodes sibiricus KOKUJEV, 1903

Apanteles exilis (HALIDAY, 1834)

Apanteles sqaboi PAPP, 1972

Cotesia memnon (NIXON, 1974)

Diolcogaster claritibia (PAPP, 1959)

Opius reptantis FISCHER, 1957

Vipio tentator (ROSSI, 1790) There is some information that these seven species are recorded from Germany, but the original sources can not now at present be traced (CvA).

Phaenocarpa riphaeica TOBIAS, 1986: Recorded from Germany (BELOKOBYLSKIJ 1998 
[\#11]), but the origin of this information is uncertain and may be an error (SB). Atanycolus initiator (FABRICIUS, 1793): There are only old records (NEES, HERRICHSCHÄFFER, RATZEBURG) of this species that seem to refer usually to $A$. genalis (THOMSON) (= A. initiator auct.).

Apanteles arcuatus TELENGA, 1955: Identification of the ZSM specimen doubtful, other records are from Russian Far East (SB).

\section{Species deleted from the German checklist}

The following species were recorded from Germany, but these records are based on errors (i. e. localities outside of Germany or misidentifications):

Aleiodes periscelis (REINHARD, 1863) (type locality "Wien", Austria)

Ahysia divergens BENGTSSON, 1926 (type locality "Maltsch" = Malczyce, Poland)

Apanteles seripbia NixON, 1972 ("Germany: Südtirol" = Italy)

Gyanopterus torkai (PAPP, 1971) (type locality "Neustadt Oberschlesien" = Poland)

Dacnusa annulata (NEES, 1834) (type locality Sudetes = Czech Republic)

Dacnusa gallarum (RATZEBURG, 1852) (type locality "Danzig" = Gdansk, Poland)

Dinotrema castaneum (NEES, 1834) (type locality Sudetes = Czech Republic)

Pambolus brevipennis (KIEFFER, 1905)

Perilitus deceptor (WeSMAas, 1835) (see HAESELBART'H 1988 [\# 31])

Tracbyusa bucephala PAPP, 1967

\section{Species incertae sedis}

The original genera are given in brackets [].

Apbidius constrictus (NEES, 1811)[Bracon]

Aphidius diminuens NEES, 1834

Aphidius flavidens RATZEBURG, 1844

Apbidius melanocephalus (NEES, 1811) [Bracon]

Aphidiuspanzerii RONDANI, 1848

Aphidius planistipes NEES, 1834

Ascogaster contractus (RATZEBURG, 1848)[Chelonus]

Ascogaster tersus REINHARD, 1867

Bracon ater NEES, 1811

Bracon dimidiatus NEES, 1811, nec SPINOLA, 1808; not available

Chelonus atriceps RATZEBURG, 1844

Chelonus quadridens HERRICH-SCHÄFFER, 1838

Cbelonus similis RATZEBURG, 1844

Coloboma nigrina FÖRSTER, 1862; according to the type an Aspilota species (CVA).

Leiopbron ornatus (MARSHALL, 1887) [Euphorus]

Leiopbron mitis HALIDAY, 1833 (The type - most likely a deformed specimen - is lost. CVA)

Meteorus effeminatus RUTHE, 1862

Orgilus annulator (NEES, 1812) [Microdus]

Orgilus laevigator (NEES, 1812) [Microdus] 


\section{Orgilus nigripennis (DAHL, 1912) [Eubadiron] \\ Orthostigma praedo (FÖRSTER, 1862) [Delocarpa] \\ Perilitus distinguendus HERRICH-SCHÄFFER, 1838 \\ Perilitus erythrogaster HERRICH-SCHÄFFER, 1838 \\ Praon emacerator (NEES, 1834) [Bracon] \\ Rogas praerogator (NEES, 1812) [Bracon] \\ Ascogaster similis (NEES, 1816) [Sigalphus]}

\section{References for German records}

[BMNH] The Natural History Museum (formerly: British Museum, Natural History), London, United Kingdom

[DEI] Deutsches Entomologisches Institut im ZALF, Eberswalde

[RMNH] Nationaal Natuurhistorische Museum ("Naturalis") [formerly Rijksmuseum van Natuurlijke Historie], Leiden, Netherlands

[SCHNEE] Collection of H. SCHNEE, Markleeberg

[STUTTG] Staatliches Museum für Naturkunde, Stuttgart

[ZMHUB] Museum für Naturkunde, Humboldt Universität, Institut für Systematische Zoologie, Berlin

[ZSM] Zoologische Staatssammlung, Munich

[\# 01] Achterberg, C. VAN \& KenIS, M. 2000: The Holarctic species of the subgenus Allodorus Foerster s.s. of the genus Eubarus NeEs (Hymenoptera: Braconidae). - Zoologische Mededelingen, Leiden 73: $427-455$

[\# 02] ACHTERBERG, C. vAN 1979: A Revision of the Subfamily Zelinae auct. (Hymenoptera, Braconidac). - Tijdschrift voor Entomologie, Leiden 122(7): 241-479

[\# 03] ACHTERBERG, C. VAN 1987: Revision of the European Helconini (Hymenoptera: Braconidac: Helconinae). - Zoologische Mededelingen, Leiden 61(18): 263-285.

[\# 04] ACHTERBERG, C. VAN 1988: Revision of the subfamily Blacinae FoERSTER (Hymenoptera, Braconidae). - Zoologische Verhandelingen, Leiden 249: 1-324.

[\# 05] ACHTERBERG, C. VAN 1988: The genera of the Aspilota-group and some descriptions of fungicolous Alysini from the Netherlands (Hymenoptera: Braconidae: Alysiinae). - Zoologische Verhandelingen, Leiden 247: 1-88.

[\# 06] Achterberg, C. VAN 1989: Revision of the subtribe Monoctonina MackaUer sensu stricto (Hymenoptera: Braconidae: Aphidiinae). - Zoologische Mededelingen, Leiden 63(1): 1-22

[\# 07] Achterberg, C. VAN 1990: Revision of the Western Palaearctic Phanerotomini (Hymenoptera: Braconidae). - Zoologische Verhandelingen, Leiden 255: 1-106.

[\# 08] ACHTERBERG, C. VAN 1993: Revision of the subfamily Macrocentrinae FOERSTER (Hymenoptera:Braconidae) from the Palaearctic region. - Zoologische Verhandelingen, Leiden 286: $1-110$.

[\# 09] ACHTERBERG, C. VAN \& HAESELBARTH, E. 1983: Revisionary notes on the European species of Macrocentrus CuRTIS sensu stricto (Hymenoptera: Braconidae). - Entomofauna, Linz. 4(2): 37-59.

[\# 10] Adisu, B.; STARÝ, P.; FreIER, B. \& BÜTTTER, C. 2002: Apbidius colemani Vier. (Hymenoptera, Braconidae, Aphidiinae) detected in cereal fields in Germany. - Anzeiger für Schädlingskunde. Journal of Pest Science, Berlin 75(4): 89-94

[\# 11] BelokobylskIJ, S. A. 1998: [Alysinae]. - Opredelitel' nasekomych Dalnego Vostoka Rossii, Vladivostok 4(3): 163-411

[\# 12] ČAPEK, M. \& ACHTERBERg, C. van 1992: A revision of the genus Microtypus Ratzeburg (Hymenoptera: Braconidae). - Zoologische Mededelingen, Leiden 66(21): 323-338. 
[\# 13] FisCHER, M. 1971: Untersuchungen über die europäischen Alysiini mit besonderer Berücksichtigung der Fauna Niederösterreichs (Hymenoptera, Braconidae). - Polskie Pismo Entomologiczne, Warszawa, Wrocław 41(1): 19-160.

[\# 14] Fischer, M. 1971: World Opiinae (Hym. Braconidae). - In: Delucchi, V. \& Remaudière, G. (Hrsg.): Index of Entomophagous Insects 5: 1-189.

[\# 15] FISCHER, M. 1972: Hymenoptera, Braconidae (Opiinae I). - Das Tierreich, Berlin 91: I-XII, 1-620, 463

[\# 16] FisCHER, M. 1973: Aspilota-Wespen aus der weiteren Umgebung von Admont (Hym., Braconidae, Alysiinae). - Mitteilungen der Abteilung für Zoologie am Landesmuseum Joanneum, Graz 2(3): $137-167$

[\# 17] Fischer, M. 1974: Aspilota-Arten gezogen aus Phoriden (Hymenoptera: Braconidae). - Bolletino del Laboratorio di Entomologia Agraria "Filippo Silvestri" Portici 31: 253-264

[\# 18] FISCHER, M. 1985: Eine neue Aspilota-Art aus Bayern (Hymenoptera, Braconidae, Alysiinae). Entomofauna, Linz 6(18): 233-238

[\# 19] FIscheR, M. 1993: Zur Formenvielfalt der Kieferwespen der Alten Welt: Über die Gattungen Synaldis FOERSTER, Trisynaldis FISCHER und Kritscberysia FISCHER gen. nov. (Hymenoptera, Braconidae, Alysiinae). - Annalen des Naturhistorischen Museums in Wien. Ser. B: Botanik und Zoologie, Wien 94/95: 451-490

[\# 20] FISCHER, M. 1997: Die paläarktischen Opïnae (Madenwespen) der Zoologischen Staatssammlung München (Hymenoptera, Braconidae). - Entomofauna, Ansfelden 18(14): 137-196.

[\#21] GÄRDENFors, U. 1986: Taxonomic and biological revision of Palaearctic Ephedrus HALIDAY (Hymenoptera: Braconidae, Aphidiinae). - Entomologica Scandinavica, Supplement, Copenhagen 27: $1-95$

[\# 22] Griffiths, G. C. D. 1964: The Alysiinae (Hym. Braconidae) parasites of Agromyzidae (Diptera). I. General questions of taxonomy, biology and evolution. - Beiträge zur Entomologie, Berlin 14(78): 823-914.

[\# 23] GRIfFItHS, G. C. D. 1967: The Alysiinae (Hym. Braconidae) parasites of Agromyzidae (Diptera). II. The parasites of Agromyza FALLÉN. - Beiträge zur Entomologie, Berlin 16[1966](5-6): 551-605

[\# 24] GRIfFItHS, G. C. D. 1967: The Alysiinae (Hym. Braconidae) parasites of Agromyzidae (Diptera). III. The parasites of Paraphytomyza ENDERLEIn, Pbytagromyza HeNDEL and Phytomyza FAllén. Beiträge zur Entomologie, Berlin 16[1966](7-8): 775-951

[\# 25] GriffithS, G. C. D. 1967: The Alysiinae (Hym. Braconidae) parasites of Agromyzidae (Diptera). IV. The parasites of Hexomyza ENDERLEIN, Melanagromyza HENDEL, Ophiomyia BRASCHNIKOV and Napomyza WeSTwOOD. - Beiträge zur Entomologie, Berlin 17(5-8): 653-696

[\# 26] GrIfFItHS, G. C. D. 1968: The Alysiinae (Hym. Braconidae) parasites of Agromyzidae (Diptera). $\mathrm{V}$. The parasites of Liriomyza MIK and certain small genera of Phytomyzinae. - Beiträge zur Entomologie, Berlin 18(1-2): 5-62

[\# 27] GRIfFItHS, G. C. D. 1968: The Alysiinae (Hym. Braconidae) parasites of Agromyzidae (Diptera). VI. The parasites of Cerodontha RONDANI s. 1. - Beiträge zur Entomologie, Berlin 18(1-2): 63-152

[\# 28] GRIffrthS, G. C. D. 1984: The Alysinae (Hym. Braconidae) parasites of Agromyzidae (Diptera). VII. Supplement. - Beiträge zur Entomologie, Berlin 34(2): 343-362.

[\# 29] HAESElBARTH, E. 1967: Zur Kenntnis der palaearktischen Arten der Gattung Coeloides WeSMAEL (Hymenoptera, Braconidae). - Mitteilungen der Münchner Entomologischen Gesellschaft, München 57: 20-53

[\# 30] HAESELBARTH, E. 1985: Bestimmungsliste entomophager Insekten Nr. 10. - Bulletin SROP. Organisation Internationale de Lutte Biologique contre les animaux et les plantes nuisibles. Section Regionale Quest Palearctique, Montfavet 8(4): 1-61

[\# 31] HAeselbartH, E. 1988: Zur Braconidengattung Townesilitus HAESELBARTH \& LOAN, 1983. Entomofauna, Linz 9(23): 429-460

[\# 32] HAESELBARTH, E. 1989: Bestimmungsliste entomophager Insekten Nr. 11. - Bulletin SROP. Organisation Internationale de Lutte Biologique contre les animaux et les plantes nuisibles. Section Regionale Quest Palearctique, Montfavet 12(7): 1-63 
[\#33] HAESELBARTH, E. 1996: Rilipertas gen. nov., eine neue Gattung der Euphorinae (Hymenoptera, Braconidae). - Entomofauna, Ansfelden 17(26): 397-412.

[\# 34] HAEsELBARTH, E. 1999: Zur Braconiden-Gattung Perilitus NEES, 1818. 2. Beitrag: Die Arten mit ausgebildetem ersten Cubitus-Abschnitt (Insecta, Hymenoptera, Braconidae). - Mitteilungen der Münchner Entomologischen Gesellschaft, München 89: 11-46

[\# 35] HERRICH-SCHÄFFER, G. A. W. (1838): Faunae Insectorum Germanicae initia oder Deutschlands Insecten. Regensburg 156: 1-24

[\# 36] HERRICH-SChÄpFER, G. A. W. (1838): Faunae Insectorum Germanicae initia oder Deutschlands Insecten, Regensburg 154: 1-24

[\# 37] HeydenanN, B. 1967: Die biologische Grenze Land-Meet im Bereich der Salzwiesen. - Franz Steiner Verlag GmbH, Wiesbaden, 200 p.

[\#38] Huddleston, T. 1980: A revision of the western Palaearctic species of the genus Meteorus (Hymenoptera: Braconidae). - Bulletin of the British Museum (Natural History). Entomology series, London 41(1): $1-58$

[\# 39] Huddeston, T. 1984: The Palaearctic species of Ascogaster (Hymenoptera: Braconidae). - Bulletin of the British Museum (Natural History). Entomology series, London 49(5): 341-392

[\# 40] KeTtNeR, F. W. 1965: Deutsche Braconiden und thre Wirte (Hymenoptera). - Verhandlungen des Vereins für naturwissenschaftliche Heimatforschung, Hamburg 36: 102-146

[\# 41] KÖNIG, R. 1972: Zur Systematik, Faunistik, Phänologie und Ölkologie mitteleuropäischer Btaconiden (Hymenoptera) (1) - Faunistisch-ökologische Mitteilungen, Kiel 4: 85-106

[\# 42] Königsmann, E. 1960: Revision der paläarktischen Arten der Gattung Idiasta. 3. Beitrag zur systematischen Bearbeitung der Alysiinae (Hymenoptera:Braconidae). - Beiträge zur Entomologie, Berlin 10: 624-654

[\# 43] Königsmann, E. 1969: Beitrag zur Revision der Gattung Orthostigma (Hymenoptera, Braconidae). - Deutsche entomologische Zeitschrift, Neue Folge, Berlin 16(1-3): 1-53

[\# 44] LOAN, C. C. 1974: The European species of Leiopbron NEES and Peristenus FoERSTER (Hymenoptera: Braconidae, Euphotinae). - The Transactions of the Royal Entomological Society of London, London 126(2): 207-238

[\# 45] MACKauer, M. \& StarÝ, P. 1967: Hym. Ichneumonoidea. World Aphidiidae. - In: Delucchi, V. \& Remaddiere, G. (eds.): Index of Entomophagous Insects. - Le François, Paris: 195 pp.

[\# 46] MACKAUER, M. 1968: Aphidiidae. - Hymenopterorum Catalogus (nova editio) - 's-Gravenhage 3: $1-103$

[\# 47] NIXON, G. E. J. 1943: A Revision of the European Dacnusini (Hym., Braconidae, Dacnusinae). Entomologist's Monthly Magazine, Fourth Series, London 79(7): 159-168

[\# 48] NixON, G. E. J. 1944: A Revision of the European Dacnusini (Hym., Braconidae, Dacnusinae). Entomologist's Monthly Magazine, Fourth Series, London 80(11): 249-255

[\# 49] NixON, G. E. J. 1944: A Revision of the European Dacnusini (Hym., Braconidae, Dacnusinae). Entomologist's Monthly Magazine, Fourth Series, London 80(4-5): 88-108

[\# 50] NIXON, G. E. J. 1945: A Revision of the European Dacnusini (Hym., Braconidae, Dacnusinae). Entomologist's Monthly Magazine, Fourth Series, London 81(8-9): 189-204

[\# 51] NIXON, G. E. J. 1946: A Revision of the European Dacnusini (Hym., Braconidae, Dacnusinae). Entomologist's Monthly Magazine, Fourth Series, London 82(11-12): 279-300

[\# 52] Nixon, G. E. J. 1948: A Revision of the European Dacnusini (Hym., Braconidae, Dacnusinae). Entomologist's Monthly Magazine, Fourth Series, London 84(9-10): 207-224

[\# 53] Nrxon, G. E. J. 1949: A Revision of the European Dacnusini (Hym., Braconidae, Dacnusinae). Entomologist's Monthly Magazine, Fourth Series, London 85(12): 289-298

[\# 54] NIXON, G. E. J. 1954: A Revision of the European Dacnusini (Hym., Braconidae, Dacnusinae). - Entomologist's Monthly Magazine, Fourth Series, London 90(11-12): 257-290

[\# 55] Nixon, G. E. J. 1968: A Revision of the Genus Microgaster LATRerlle (Hymenoptera: Braconidae). - Bulletin of the British Museum (Natural History). Entomology series, London 22(2): 31-72 
[\# 56] NIXON, G. E. J. 1972: A revision of the north-western European species of the laevigatus-group of Apanteles FÖRSTER (Hymenoptera, Braconidae). - Bulletin of Entomological Research, London 61: 701-743

[\# 57] NixON, G. E. J. 1973: A revision of the north-western European species of the vitripennis, pallipes, octonarius, triangulator, fraternus, formosus, parasitellae, metacarpalis and circumscriptus-groups of Apanteles FÖRSTER (Hymenoptera, Braconidae). - Bulletin of Entomological Research, London 63: 169-228

[\# 58] NIXON, G. E. J. 1974: A revision of the north-western European species of the glomeratus-group of Apanteles FörSTER (Hymenoptera, Braconidae). - Bulletin of Entomological Research, London 64: $453-524$

[\# 59] NIXON, G. E. J. 1976: A revision of the north-western European species of the merula, lacteus, vipio, ultor, ater, butalidis, popularis, carabonarius and validus-groups of Apanteles FÖRSTER (Hymenoptera, Braconidae). - Bulletin of Entomological Research, London 65[1975-76]: 687.732

[\# 60] Nixon, G. E. J. 1986: A revision of the European Agathidinae (Hymenoptera: Braconidae). - Bulletin of the British Museum (Natural History). Entomology series, London 52(3): 183-242, 68 Fig.

[\# 61] PAPP, J. 1966: A Synopsis of the Bracon F. Species of the Carpathian Basin (Hymenoptera, Braconidae). I. Subgenus Glabrobracon FAHR. - Annales historico-naturales Musei Nationalis Hungarici, Budapest 58: 373-394.

[\# 62] PAPp, J. 1968: A Synopsis of the Bracon Fabr. Species of the Carpathian Basin, Central Europe (Hymenoptera, Braconidae). Il. Subgenus Bracon Fabr. - Annales historico-naturales Musei Nationalis Hungarici, Budapest 60: 191-211.

[\# 63] PAPP, J. 1969: A Revision of THOMSON's Species of Bracon FABR. (Hym. Braconidae). - Opuscula Entomologica, Lund 34: 177-205

[\# 64] PAPP, J. 1969: A Synopsis of the Bracon FABR. Species of the Carpathian Basin (Hymenoptera, Braconidae). III. Subgenus Lucobracon (FAHR.) TOB. - Annales historico-naturales Musei Nationalis Hungarici, Budapest 61: 317-335.

[\# 65] PAPP, J. (1976): Key to the European Microgaster LATR. species, with a new species and taxonomical remarks (Hymenoptera: Braconidae, Microgasterinae). - Acta Zoologica Ácademiae Scientiarum Hungaricae, Budapest 22(1-2): 97-117

[\# 66] PAPP, ]. 1978: A survey of the European species of Apanteles FÖRST. (Hymenoptera, Braconidae: Microgastrinae), II. The laevigatus-group, 1. - Annales historico-naturales Musei Nationalis Hungarici, Budapest 70: 265-301

[\# 67] PAPp, J. 1979: A survey of the European species of Apanteles FÖRST. (Hymenoptera, Braconidae: Microgastrinae) III. The laevigatis-group, 2. - Annales historico-naturales Musei Nationalis Hungarici, Budapest 71: 235-250.

[\# 68] PAPP, J. 1981: A survey of the European species of Apanteles FÖRST. (Hymenoptera, Braconidae: Microgastrinae), V. The lacteus-, longipalpis, ultor, butalidis- and vipio-group. - Annales historico-naturales Musei Nationalis Hungarici, Budapest 73: 263-291.

[\# 69] PAPP, J. 1982: A survey of the European species of Apanteles FÖRST. (Hymenoptera, Braconidae: Microgastrinae), VI. The laspeyresiella-, merula-, falcatus- und validus-group. - Annales historico-naturales Musei Nationalis Hungarici, Budapest 74: 255-267.

[\# 70] PAPP, J. 1982: Taxonomical and faunistical novelties of the Opiinae in the Arctogaea (Hymenoptera, Braconidae). - Annales historico-naturales Musei Nationalis Hungarici, Budapest 74: 241-253

[\# 71] PAPP, J. 1983: A survey of the European species of Apanteles FÖST. (Hymenoptera, Braconidae: Microgastrinae), VII. The carbonarius-, circumscriptus-, fraternus-, pallipes-, parasitellae-, yitripennis-, liparidis, octonarius-and thomsoni-group. - Annales historico-naturales Musei Nationalis Hungarici, Budapest 75: $247-283$

[\# 72] PAPP, J. (1984): Palaearctic Species of Microgaster LATREILLE (Microplitis FÖRSTER) with Description of Seven New Species (Hymenoptera, Braconidae, Microgastrinae). - Entomologische Abhandlungen Aus dem Staatliches Museum für Tierkunde in Dresden, Dresden 47[1983](7): 95-140, 148 Fig.

[\# 73] PAPp, J. 1984: A survey of the European species of Apanteles FörST. (Hymenoptera, Braconidac: Microgastrinae), VIII. The metacarpalis-, formosus-, popularis- and suevus-group. - Annales historiconaturales Musei Nationalis Hungarici, Budapest 76: 265-295. 
[\# 74] PAPp, ]. 1986: A survey of the European species of Apanteles FÖRST. (Hymenoptera, Braconidae: Microgastrinae), IX. The glomeratus-group. - Annales historico-naturales Musei Nationalis Hungarici, Budapest 78: 225-247.

[\# 75] PAPP, J. 1987: A survey of the European species of Apanteles FörST. (Hymenopteta, Braconidae: Microgastrinae), X. The glomeratus-group 2 and the cultellatus-goup. - Annales historico-naturales Musei Nationalis Hungarici, Budapest 79: 207-258.

[\# 76] PAPP,J. 1990: A Revision of THOMSON's Micracbelonus species (Hymenoptera: Braconidae, Cheloninae). - Acta Zoologica Academiae Scientiarum Hungaricae, Budapest 36(3-4): 295-317.

[\# 77] PAPP, J. 1997: Revision of the Chelonus species described by A. D. DAHLBOM (Hymenoptera: Braconidae, Cheloninae). - Acta Zoologica Academiae Scientiarum Hungaricae, Budapest 43(1): 1-19

[\# 78] PAPP, J. 1992: New braconid wasps (Hymenoptera, Braconidae) in the Hungarian Natural History Museum, 3. - Annales historico-naturales Musei Nationalis Hungarici, Budapest 84: 129-160

[\# 79] PAPP, J. 1995: Revision of C. WESMAEL's Chelonus species (Hymenoptera Braconidae Cheloninae). - Bulletin de l'Institut Royal des Sciences Naturelles de Belgique 65: 115-134

[\# 80] PAPP, J. 1998: New braconid wasps (Hymenoptera, Braconidae) in the Hungarian Natural History Museum 6. - Annales historico-naturales Musei Nationalis Hungarici, Budapest 90: 221-256

[\# 81] PApP, J. 1998: Two new braconid species from Switzerland (Hymenoptera, Braconidae). - Mitteilungen der Schweizerischen Entomologischen Gesellschaft, Zürich 71: 133-139

[\# 82] PAPp, J. 1999: Braron (Glabrobracon) dilatus sp. n. from Iran and Iraq with taxonomic remarks on several relative species (Hymenoptera: Braconidae, Braconinae). - Folia Entomologica Hungarica, Budapest 60: 269-282

[\# 83] PAPP, J. 2000: First synopsis of the species of the obscurator species-group, genus Bracon, subgenus Glabrobracon (Hymenoptera: Braconidae, Braconinae). - Annales historico-naturales Musei Nationalis Hungarici, Budapest 92: 229-264.

[\# 84] PAPP, J. \& OEHLKE, J. 1982: Zur Brackwespenfauna der Insel Hiddensee. Ein Beitrag zur Fauna von Naturschutzgebieten der DDR (Insecta, Hymenoptera, Braconidae). - Faunistische Abhandlungen Staatliches Museum für Tierkunde Dresden, Dresden 9[1981](2 (17)): 185-193

[\# 85] RUTHE, J. F. 1862: Deutsche Braconiden. Drittes Stück. - Berliner Entomologische Zeitschrift, Berlin 6: 1-58

[\# 86] SHENEFELT, R. D. 1969-1978: Braconidae 1-10. - Hymenopterorum Catalogus (nova editio) 4-7, 9-13, 15: 1-1872. - ‘s-Gravenhage (10: SHENEFELT, R. D. \& MARSH, P. M.; Index - Braconidae 11: 16 ACHTERBERG C. VAN \& SHENEFELT, R. D., 1980, 384 pp.)

[\# 87] Simbolotti, G. \& AChterberg, C. VAN 1999: Revision of the West Palaearctic species of the genus Agatbis LATREILLE (Hymenoptera: Braconidae). -Zoologische Verhandelingen, Leiden 325: $1-167$

[\# 88] SMmbolotti, G.; ACHTERBERG, C. VAN 1992: Revision of the West Palaearctic species of the genus Bassus FABRICIUS (Hymenoptera: Braconidae). - Zoologische Verhandelingen, Leiden 281: 1-80

[\# 89] TAeger, A. 1989: Die Orgilus-Arten der Paläarktis (Hymenoptera, Braconidae). - Akademie der Landwirtschaftswissenschaften der DDR, Berlin [1988]: 1-260

[\# 90] Tobias, V. I.; JAKImavicius, A. B. \& KirIYAK, I. G. (1986): III Perepontschatokrylye. - In: Medvedev, G. S. 1986: Opredelitel' nasekomych evropejskoj tschasti SSSR. - Nauka, Leningrad 3(5): 312 p., 189 pl.

[\# 91] WharTON, R. A. 1986: The braconid genus Alysia (Hymenoptera, Braconidae): a description of the subgenera and a revision of the subgenus Alysia. - Systematic Entomology, Oxford, London u.a. 11: $453-504$.

[\# 92] WHARTON, R. A. 1988: The braconid genus Alysia (Hymenoptera, Braconidae): a revision of the subgenus Anarcha. - Contributions of the American Entomological Institute, Gainesville 25(2): $1-69$.

[\# 93] HORSTMANN, K. 1999: Zur Interpretation der von THUNBERG in der Gattung Icbneamon LINNAEUS beschriebenen oder benannten Arten (Hymenoptera). - Zeitschrift der Arbeitsgemeinschaft Österreichischer Entomologen, Wien 51: 65-75 
[\# 94] PAPP, J. 1988: A survey of the European species of Apanteles FÖRST. (Hymenoptera, Braconidae: Microgastrinae), XI. "Homologization" of the species-groups of Apanteles s. l. with MASON's generic taxa. Checklist of genera. Parasitoid / host list 1. - Annales historico-naturales Musei Nationalis Hungarici, Budapest 80: 145-175

[\# 95] PAPP, J. 1975: Contribution to the braconid fauna of Yugoslavia (Hymenoptera: Braconidae): 2. - Folia Entomologica Hungarica, Budapest 28(2): 301-319

[\# 96] QUICKE, D. L. J.; VAN ACHTERBERG, K. \& GODFRAY, H. C. J. 1997: Comperative morphology of the venom gland and reservoir in opiine and alysiine braconid wasps (Insecta, Hymenoptera, Braconidae). - Zoologica Scripta, Oxford u. a. 26(1): 23-50

[\# 97] SHAW, S. 1992: Seven new North American species of Neoneurus (Hymenoptera: Braconidae). Ptoceedings of the Entomological Society of Washington, Washington 94(1): 26-47

[\# 98] PAPP, J. 1983: A survey of the Braconid Fauna of the Hortobágy National Park (Hymenoptera, Braconidae) II. - The fauna of the Hortobágy National Park 2: 315-337

\section{Register of species and synonyms}

Valid species marked with an asterisk $\left(^{*}\right)$. The numbers refer to the numbers used in the checklist above.

abbreviator Nees (Bracon) $* \rightarrow 548$

abbreviator Ratzeburg (Orgilus) $* \rightarrow 1418$

abdita Haliday (Dacnusa) $* \rightarrow 227$

abdominalis Fabricius (Macrocentrus) $\rightarrow 1031$

abdominalis Nees (Diolcogaster) $* \rightarrow 1115$

abdominalis Zetterstedt (Coeloides) $* \rightarrow 650$

abdominator Dahlbom (Ascogaster) $* \rightarrow 680$

abdominator Nees (Alysia) $\rightarrow 62$

abdominator Nees (Meteorus) $* \rightarrow 849$

abieticola van Achterberg \& Kenis (Eubazus) $* \rightarrow 480$

abietis Marshall (Pauesia) $* \rightarrow 439$

abietis Ratzeburg (Baeacis) $* \rightarrow 982$

abila Nixon (Dolichogenidea) $\rightarrow 1126$

abjecta Marshall (Cotesia) $* \rightarrow 1061$

abnormis Wesmael (Colastes) $* \rightarrow 950$

abnormis Wesmael (Eurytenes) $* \rightarrow 1301$

aboletus Papp (Polemochartus) $* \rightarrow 347$

abrota Nixon (Chorebus) $* \rightarrow 97$

abscissor Nees (Bracon) $\rightarrow 603$

abscissus Ratzeburg (Bassus) $\rightarrow 26$

abscissus Thomson (Opius) $\rightarrow 1355$

abscissus Thomson (Meteorus) $* \rightarrow 850$

absinthii Bignell (Praon) $* \rightarrow 451$

absinthii Marshall (Aphidius) $\rightarrow 376$

absintii Marshall misspell. (Aphidius) $\rightarrow 376$

acalephae Marshall (Misaphidus) $* \rightarrow 426$

acasta Nixon (Glyptapanteles) * $\rightarrow 1158$

accincta Haliday (Leiophron) $* \rightarrow 830$

acco Nixon (Chorebus) $\rightarrow 175$

aceris Haliday (Trioxys) $\rightarrow 468$

achterbergi Taeger (Orgilus) $* \rightarrow 1419$ aciculata Förster (Symphanes) $* \rightarrow 354$

aciculatus Ratzeburg (Triaspis) $\rightarrow 535$

aciculatus Reinhard (Polystenus) $\rightarrow 775$

aciculatus Ruthe (Rhaconotus) * $\rightarrow 776$

aconiti Griffiths (Exotela) $* \rightarrow 291$

actaphractus Śnoflak (Schizoprymnus) * $\rightarrow 513$

aculeator Marshall (Dolopsidea) $\rightarrow 1443$

acuminata Reinhard (Cotesia) $* \rightarrow 1062$

acuminata Szépligeti (Phanerotoma) $* \rightarrow 739$

acuminatus Herrich-Schäffer (Chelonus) $* \rightarrow 699$

acutus Tobias (Proacrisis) $* \rightarrow 974$

adaequator Förster (Opius) $\rightarrow 1371$

addendus Fischer (Opius) $\rightarrow 1356$

adducta Haliday (Dacnusa) $* \rightarrow 228$

adjaricus Tobias (Microchelonus) $\rightarrow 726$

adectus Szépligeti (Bracon) $\rightarrow 590$

adjuncta Nees (Dolichogenidea) $* \rightarrow 1122$

adjunctus Marshall (Apanteles) $\rightarrow 1054$

adoxophyesi Minamikawa (Meteorus) $\rightarrow 863$

aduncus Ruthe (Microplitis) $* \rightarrow 1211$

adveniens Fischer (Opius) $\rightarrow 1355$

aegeriae Rohwer (Macrocentrus) $\rightarrow 1032$

aemulus Haliday (Utetes) $* \rightarrow 1406$

aemulus Ruthe (Perilitus) $* \rightarrow 884$

aeolus Nixon (Iconella) $* \rightarrow 1179$

aequalis Thomson (Bracon) $\rightarrow 640$

aequator Herrich-Schäffer (Eubazus) $* \rightarrow 481$

aequator Nees (Helconidea) $\rightarrow 1003$

aethiopoides Loan (Perilitus) $* \rightarrow 885$

aethiops auct. (Perilitus) $\rightarrow 885$

aethiops Haliday (Opius) $* \rightarrow 1303$ 
aethiops Nees (Perilitus) $* \rightarrow 886$

affiniqades Shenefelt (Macrocentrus) $\rightarrow 1025$

affinis Hedwig (Macrocentrus) $\rightarrow 1025$

affinis Herrich-Schäffer (Aleiodes) $* \rightarrow 1447$

affinis Herrich-Schäffer (Triaspis) $* \rightarrow 525$

affinis Mackauer (Trioxys) $\rightarrow 473$

affinis Nees (Chorebus) $\rightarrow 157$

affinis Nees (Cotesia) $* \rightarrow 1063$

affinis Quilis (Aphidius) $\rightarrow 385$

affinis Wesmael (Diospilus) $\rightarrow 993$

affinis Wesmael (Earinus) $\rightarrow 44$

affinis Wesmael (Meteorus) $* \rightarrow 851$

agraules Nixon (Chorebus) $* \rightarrow 98$

agricolorator Linné (Cenocoelius) $\rightarrow 678$

agromyzicola Fischer (Opius) $* \rightarrow 1304$

ahrburgensis Fischer (Aspilota) $* \rightarrow 82$

albanica Fischer (Agathis) $\rightarrow 10$

albicornis Ruthe (Meteorus) $\rightarrow 857$

albicostellae Fischer (Agathis) $\rightarrow 13$

albicoxis Marshall (Opius) $\rightarrow 1336$

albiditarsis Curtis misspell. (Zele) $\rightarrow 936$

albiditarsus Curtis (Zele) $* \rightarrow 936$

albiflagellaris Starý (Pauesia) $\rightarrow 441$

albimarginis Griffiths (Chorebus) * $\rightarrow 99$

albipennis Nees (Bracon) $* \rightarrow 549$

albipennis Nees (Dolichogenidea) $* \rightarrow 1123$

albipes Haliday (Chorebus) $* \rightarrow 100$

albiscutis Telenga (Atanycolus) $\rightarrow 541$

albitarsis Reinhard (Ascogaster) $* \rightarrow 681$

albitarsus Haliday (Zele) $\rightarrow 936$

albitarsus Nees (Zele) $\rightarrow 936$

albitarsus Reinhard misspell. (Ascogaster) $\rightarrow 681$

albitibia Herrich-Schäffer (Aleiodes) $* \rightarrow 1448$

alboannulatus Szépligeti (Microchelonus) $\rightarrow 733$

alebion Nixon (Microgaster) $* \rightarrow 1184$

alecto Morley (Chorebus) * $\rightarrow 101$

alexis Curtis (Microgaster) $\rightarrow 1197$

aliphaera Nixon misspell. (Glyptapanteles) $\rightarrow 1159$

aliphera Nixon (Glyptapanteles) $* 1159$

alpestris Griffiths (Dacnusa) $* \rightarrow 229$

alternans Enderlein (Cremnops) $\rightarrow 41$

alternata Ruthe (Meteorus) $\rightarrow 856$

alternator Nees (Aleiodes) $* \rightarrow 1449$

alternipes Nees (Acampsis) $* \rightarrow 1487$

altimontanus Fischer (Opius) $\rightarrow 1389$

alutaceus Szépligeti (Bracon) $\rightarrow 597$

aluteceus Szépligeti misspell. (Bracon) $\rightarrow 597$

alvearia Fabricius (Diolcogaster) $* \rightarrow 1116$

alveator Thunberg (Diolcogaster) $\rightarrow 1116$

amabilis Nixon (Cotesia) $\rightarrow 1106$

amarellae Fischer (Opius) $* \rightarrow 1305$ amauromyzae Griffiths (Chorebus) $* \rightarrow 102$

amaurosomae Telenga (Synelix) $\rightarrow 360$

ambiguus auct. (Lysiphlebus) $\rightarrow 420$

ambiguus Haliday (Adialytus) $* 371$

ambiguus Nees (Schizoprymnus) $* \rightarrow 514$

ambiguus Ruthe (Meteorus) $\rightarrow 853$

ambiguus Ruthe (Microgaster) $\rightarrow 1198$

ambiguus Thomson (Schizoprymnus) $\rightarrow 516$

ambiguus Wesmael (Schizoprymnus) $\rightarrow 518$

ambiguus Wesmael (Opius) $* 1306$

amentorum Ratzeburg (Microgaster) $\rightarrow 1194$

amicroploides Viereck (Macrocentrus) $\rightarrow 1031$

ampliator Nees (Chorebus) $* \rightarrow 103$

amplipennis Förster (Tanycarpa) $* \rightarrow 361$

amplisignatum Fischer (Dinotrema) $* \rightarrow 275$

analis Nees (Cenocoelius) $* \rightarrow 677$

analis Nees (Cotesia) $* \rightarrow 1064$

analis Wesmael (Clinocentrus) $\rightarrow 945$

analis W'esmael (Biosteres) $* \rightarrow 1275$

anceps Curtis (Coelinius) $\rightarrow 223$

anceps Schiödte (Coelinius) $\rightarrow 223$

anchisiades Nixon (Agathis) $\rightarrow 11$

anchisiades Nixon (Protapanteles) $* \rightarrow 1256$

ancilla Haliday (Tanycarpa) $\rightarrow 362$

ancilla Nixon (Cotesia) $* \rightarrow 1065$

andromica Nixon (Protapanteles) $* \rightarrow 1257$

angelicae Haliday (Misaphidus) $* \rightarrow 427$

angelicina Griffiths (Dacnusa) $* \rightarrow 230$

anglica Marshall (Agathis) $* \rightarrow 10$

angulator Herrich-Schäffer (Eubazus) $\rightarrow 481$

angulator Nees (Praon) $\rightarrow 463$

angulator Ratzeburg misspell. (Bassus) $\rightarrow 30$

anguligena Nixon (Chorebus) $\rightarrow 142$

angustator Nees (Helcon) $* \rightarrow 999$

angustatus Herrich-Schäffer (Schizoprymnus) $* \rightarrow 515$

angustinus Ruthe misspell. (Eubazus) $\rightarrow 482$

angustula Haliday (Pentapleura) $* \rightarrow 324$

annae Enderlein (Bassus) $\rightarrow 39$

annularis Haliday (Dolichogenidea) $* \rightarrow 1124$

annularis Nees (Ascogaster) $* \rightarrow 682$

annulata Nees (Dacnusa) $* \rightarrow$ no German record

annulator Nees (Orgilus) $\rightarrow$ incertae sedis

annulatus Nees (Chelonus) $* \rightarrow 700$

annulicornis Nees (Wroughtonia) $\rightarrow 1007$

annulicornis Nees (Homolobus) $* \rightarrow 1009$

annuligaster Fulmek (Bassus) $\rightarrow 39$

annulipes Curtis (Microgaster) $\rightarrow 1209$

annulipes Herrich-Schäffer (Aleiodes) $\rightarrow 1480$

annulipes Wesmael (Chelonus) $* \rightarrow 701$

anomalus Lyle (Deuterixys) $\rightarrow 1114$

antefurcalis Belokobylskij (Hormius) $* \rightarrow 966$ 
antennalis Thomson (Schizoprymnus) $\rightarrow 518$ antennalis Thomson (Chelonus) $* \rightarrow 702$ anthomyiarum Bouché (Microgaster) $\rightarrow 1194$ anthtacina Kriechbaumer (Disophrys) $\rightarrow 42$ anthracinus Nees (Bracon) $\rightarrow 568$ antica Wollaston (Ontsira) $* 771$ antinoe Nixon (Glyptapanteles) $* \rightarrow 1160$ antione Nixon misspell. (Glyptapanteles) $\rightarrow 1160$ antipoda Ashmead (Cotesia) $\rightarrow 1099$ anuphrievi Tobias (Bassus) $\rightarrow 39$ anurus Thomson (Orgilus) $* \rightarrow 1420$ aomoriensis Matsumura (Hygroplitis) $\rightarrow 1178$ apfelbeckianus Fischer (Opius) $* 1307$ aphantus Marshall (Chorebus) $* \rightarrow 104$ aphidiiformis Ratzeburg (Achoristus) $\rightarrow$ nomen falsum aphidiperda Rondani? (Lysiphlebus) $\rightarrow 422$ aphidivorus Ratzeburg (Praon) $\rightarrow 463$ aphidum Mukerji \& Chatterjee (Diaeretiella) $\rightarrow 404$ aphidum Panzer (Diolcogaster) $\rightarrow 1116$ apicalis Curtis (Alysia) $\rightarrow 58$ apicalis Haliday (Leiophron) $* \rightarrow 831$ apicalis Reinhard (Aleiodes) $\rightarrow 1450$ apiculatus Fahringer (Aleiodes) $* \rightarrow 1450$ apii Curtis (Dapsilarthra) $* \rightarrow 268$ appellator Marshall (Pseudovipio) $\rightarrow 670$ appellator Nees (Vipio) $* \rightarrow 673$ appellator Telenga (Dolichogenidea) $* \rightarrow 1125$ apricus Schmiedeknecht (Bracon) $* \rightarrow 550$ aptenodytes Marshall (Blacus) $\rightarrow 809$ apterus Nees (Chasmodon) $* \rightarrow 96$ apterus Wesmael (Spathius) $\rightarrow 785$ aptus Papp (Apanteles) $\rightarrow 1043$ aquilegiae Marshall (Dacnusa) $* \rightarrow 231$ aquilis Mackauer misspell. (Aphidius) $\rightarrow 375$ aquilus Mackauer (Aphidius) ${ }^{*} \rightarrow 375$ aranearum Walckenaet (Cotesia) $\rightarrow 1109$ arctica Thomson (Phaenocarpa) $\rightarrow 331$ arctica Thomson (Cotesia) $* \rightarrow 1066$ arcticus Hellén (Triaspis) $\rightarrow 528$ arcticus Thomson (Aleiodes) $* \rightarrow 1451$ arcuatus Reinhard (Bassus) $* \rightarrow 24$ arcuatus Telenga (Apanteles) $* \rightarrow$ record uncertain arcuatus Thomson (Bracon) $* \rightarrow 551$ ardeaepenellae Bouché (Pholetesor) $\rightarrow 1249$ arenarius Haliday (Apanteles) $\rightarrow 1052$ arenarius Stelfox (Biosteres) $* \rightarrow 1276$ arene Nixon (Apanteles) $* \rightarrow 1040$ arenosus Szépligeti (Opius) $\rightarrow 1391$ areolaris Gerdin \& Hedqvist (Perilitus) * $\rightarrow 887$ areolaris Nees (Dacnusa) $* \rightarrow 232$ areolaris Thomson (Opius) $\rightarrow 1365$ areolaris Thomson (Microgaster) $* \rightarrow 1185$ areolatus Szépligeti (Diolcogaster) $\rightarrow 1116$ arete Fahringer (Eubazus) $\rightarrow 505$ ariadne Nixon (Agathis) $\rightarrow 23$ aridula Thomson (Protodacnusa) $* \rightarrow 350$ arisba Nixon (Pholetesor) $* 1247$ armata Wesmael (Ascogaster) $* \rightarrow 683$ armator Marshall (Helconidea) $\rightarrow 1003$ armatulus Ruthe (Blacus) $* \rightarrow 793$ armatus Wesmael (Allurus) $\rightarrow 790$ armida Nixon (Chorebus) $* \rightarrow 105$ artemesiana Fischer (Agathis) $\rightarrow 13$ artemisiellus Griffiths (Chorebus) * $\rightarrow 106$ artissima Papp (Dolichogenidea) $* 1126$ arunci Griffiths (Exotela) $* \rightarrow 292$ arundinis Haliday (Aphidius) $\rightarrow 385$ arvensis Starý (Lysaphidus) $* \rightarrow 418$ arvicola Starý (Adialytus) $\rightarrow 371$ asiatica Shestakov (Wesmaelia) $\rightarrow 935$ asini Llopsis (Phanerotoma) $\rightarrow 742$ asramenes Nixon (Chorebus) $* \rightarrow 107$ assimilis Kokujev (Agathis) $* \rightarrow 11$ assimilis Nees (Aleiodes) $* \rightarrow 1452$ asteris Haliday (Aphidius) $* \rightarrow 376$ atamiensis $A$ shmead (Ascogaster) $\rightarrow 685$ ater Curtis (Coelinidea) $\rightarrow 222$ ater Fischer (Heterospilus) $* \rightarrow 761$ ater Nees (Centistes) ${ }^{*} \rightarrow 819$ ater Nees (Bracon) $\rightarrow$ incertae sedis ater Ratzeburg (Apanteles) $\rightarrow 1057$ aterrimus Fahringer (Metaphidius) $* \rightarrow 425$ atertimus Ratzeburg (Aleiodes) $* \rightarrow 1453$ atis Nixon (Monolexis) $\rightarrow 770$ atis Nixon (Chorebus) $* \rightarrow 108$ atomos Rossi (Chelonus) $\rightarrow 709$ atra Haliday (Alysia) * $\rightarrow 50$ atrator Curtis (Meteorus) $\rightarrow 853$ atrator Nees (Bracon) $* \rightarrow 552$ atreus Nixon (Apanteles) $* \rightarrow 1041$ atriceps Ratzeburg (Chelonus) $\rightarrow$ incertae sedis atricornis Ratzeburg (Eubazus) $\rightarrow 505$ atricornis Smith (Cremnops) $\rightarrow 41$ atripes Thomson (Mictochelonus) $* \rightarrow 720$ atrorufus Nees (Bracon) $* \rightarrow 553$ auctus Haliday (Trioxys) $* \rightarrow 466$ auctus Thomson (Neoneurus) $* \rightarrow 882$ augustinus Ruthe (Eubazus) $* \rightarrow 482$ aulacorthi Stary (Aphidius) $\rightarrow 395$ aurantiaca Fahringer (Agathis) $\rightarrow 22$ aurantii Pierantoni (Lysiphlebus) $\rightarrow 422$ 
aureliae Fischer (Opius) $* \rightarrow 1308$ auriculata Fabricius (Microgaster) $* \rightarrow 1186$ aurora Haliday (Trachyusa) $* \rightarrow 369$ australis Szépligeti (Triaspis) $\rightarrow 528$ australis Thomson (Mictogaster) $* 1187$ austriaca Fischer (Dacnusa) $* \rightarrow 233$ austriacus Szépligeti (Heterospilus) $* 762$ avenae Haliday (Aphidius) $* \rightarrow 377$ avesta Nixon (Chorebus) $* \rightarrow 109$ awanomeigae Watanabe (Glyptapanteles) $\rightarrow 1167$ bachmaieri Fischer (Gnamptodon) $\rightarrow 978$ bajulus Haliday (Opius) $* \rightarrow 1309$ balcanicus Balevski (Cotesia) $\rightarrow 1104$ balteata Thomson (Dapsilarthra) $* \rightarrow 269$ balteatus Curtis (Aleiodes) $\rightarrow 1480$ balteatus Marshall (Bracon) $* \rightarrow 554$ barbatum Mackauer (Praon) $* \rightarrow 452$ barbiger Wesmael (Leiophron) $\rightarrow 841$ barcinonensis Marshall (Cyanopterus) $\rightarrow 661$ barynoti auct. (Blacus) $\rightarrow 812$ barynoti Boudier (Pygostolus) $\rightarrow 912$ barypus Marshall (Baryproctus) $* \rightarrow 547$ basalis Costa (Aleiodes) $\rightarrow 1454$ basalis Curtis (Leiophron) $\rightarrow 846$ basalis Curtis (Microchelonus) $* \rightarrow 721$ basalis Hellén (Aleiodes) $\rightarrow 1458$ basalis sensu Telenga (Microchelonus) $\rightarrow 725$ basalis Stephens (Hygroplitis) $\rightarrow 1178$ basifurcatus Fischer (Heterospilus) $\rightarrow 764$ basilewskyi Benoit (Panesia) $\rightarrow 450$ basirufus Fischer (Opius) $\rightarrow 1389$ bathyzonus Marshall (Chorebus) $* \rightarrow 110$ baudysi Quilis (Aphidius) $\rightarrow 385$ beieri Fischer (Sternaulopius) $* \rightarrow 1405$ belgicum Wesmael (Hecabolus) $\rightarrow 760$ bellina Nixon (Exotela) $\rightarrow 294$ beltrani Docavo (Ecphylus) $\rightarrow 759$ benacensis Haeselbarth (Perilitus) $* \rightarrow$ record uncertain

bensoni Nixon (Chorebus) $* \rightarrow 111$ berberidis Smith (Aphidius) $\rightarrow 384$ berolinense Ruthe (Elasmosoma) * $\rightarrow 829$ betulae Marshall (Trioxys) $* \rightarrow 467$ bianchii Telenga (Rhysipolis) $\rightarrow 977$ bicarinata Herrich-Schäffer (Ascogaster) $* \rightarrow 684$ bicarinatus Herrich-Schäffer (Coelinius) $\rightarrow 223$ bicellularis Ratzeburg (Bracon) $* \rightarrow 555$ bicingulatus Thomson (Earinus) $\rightarrow 44$ bicolor Curtis (Macrocentrus) $* \rightarrow 1021$ bicolor Förster (Phaenocarpa) $* \rightarrow 328$ bicolor Mackauer (Praon) $* \rightarrow 453$ bicolor Marechal (Coelinius) $\rightarrow 223$ bicolor Nees (Pholetesot) $* \rightarrow 1248$ bicolor Nees (Tanycarpa) $* \rightarrow 362$ bicolor Šnoflak (Phanerotoma) $\rightarrow 742$ bicolor Spinola (Aleiodes) $* \rightarrow 1454$ bicolor Szépligeti (Opius) $\rightarrow 1310$ bicolor Wesmael (Biosteres) $* \rightarrow 1277$ bicolor Wesmael (Perilitus) $* \rightarrow 888$ bicoloratus Boheman (Aleiodes) $\rightarrow 1467$ bicoloriformis Fischer (Opius) ${ }^{*} \rightarrow 1310$ bicolorina Shenefelt (Phaenocarpa) $\rightarrow 328$ bidentula Wesmael (Ascogaster) $* \rightarrow 685$ biglumis Haliday (Pambolus) $* \rightarrow 970$ bignelli Marshall misspell. (Cotesia) $\rightarrow 1067$ bignellii Marshall (Cotesia) $* \rightarrow 1067$ bilinea Lyle (Phanerotoma) $* \rightarrow 740$ bilinea sensu Telenga (Phanerotoma) $\rightarrow 741$ bimaculatus Fahringer (Schizoprymnus) $\rightarrow 524$ bimaculatus Szépligeti (Chelonus) $* \rightarrow 703$ bimaculatus Wesmael (Meteorus) $\rightarrow 880$ bipartitus Wesmael (Bracon) $* \rightarrow 556$ bipustulatus Fischer (Opius) $\rightarrow 1344$ biroi Györfi (Monoctonus) $\rightarrow 437$ bisignatus Wesmael (Bracon) $\rightarrow 609$ bisinuatus Szépligeti (Bracon) $\rightarrow 590$ bistigmaticus Morley (Neoneurus) $\rightarrow 882$ bistrigatus Roman (Aleiodes) $\rightarrow 1456$ bisulcata Herrich-Schäffer (Phanerotomella) $* \rightarrow 744$ blancardellae Bouché (Pholetesor) $\rightarrow 1249$ blanda Fahringer (Phanerotoma) $\rightarrow 742$ blandus Eady \& Clark (Macrocentrus) $* \rightarrow 1022$ blandus Haliday (Biosteres) $* \rightarrow 1278$ blantoni Fischer (Opius) $* \rightarrow 1311$ bohemani Bengtsson (Homolobus) $* \rightarrow 1010$ bohemanni Bengtsson misspell. (Homolobus) $\rightarrow 1010$ bonessi Fischer (Alloea) $* \rightarrow 47$ boops Wesmael (Myiocephalus) $* \rightarrow 881$ borealis Hellén (Cyanopterus) $\rightarrow 663$ borealis Marshall (Microplitis) $\rightarrow 1227$ borealis Thomson (Aleiodes) $* \rightarrow 1455$ borealis Zetterstedt (Biosteres) $* \rightarrow 1279$ boscai Quilis (Misaphidus) $\rightarrow 427$ bostrichorum Giraud (Coeloides) $* \rightarrow 651$ brachialis Rondani (Cardiochiles) $\rightarrow 676$ brachycera Thomson (Microplitis) $\rightarrow 1211$ brachycerus Thomson (Cotesia) $\rightarrow 1102$ brachycerus Thomson (Bracon) $* \rightarrow 557$ brachyurus Marshall (Doryctes) $\rightarrow 757$ brachyurus Telenga (Cyanopterus) $\rightarrow 660$ braconius Haliday (Colastes) $* \rightarrow 951$ brassicae Marshall (Diaetetiella) $\rightarrow 404$ 
bremeri Bengtsson (Biosteres) $* \rightarrow 1280$

breviantennatum Starý (Monoctonus) $\rightarrow 437$

breviantennatus De Stefani (Bracon) $\rightarrow 586$

brevicauda Hellén (Charmon) $\rightarrow 679$

brevicauda Hellén (Blacometeorus) $* \rightarrow 792$

brevicauda Thomson (Cyanopterus) $\rightarrow 660$

brevicauda Thomson (Meteorus) $* \rightarrow 852$

brevicauda Tobias (Noserus) $\rightarrow 969$

brevicandis Ratzeburg (Spathius) * $\rightarrow 780$

brevicaudis Reinhard (Bassus) $* \rightarrow 25$

brevicollis Haliday (Perilitus) $* \rightarrow 889$

brevicotne Nees (Dinotrema) $* \rightarrow 276$

brevicornis Curtis (Coelinidea) $\rightarrow 216$

brevicornis Haliday (Misaphidus) $* \rightarrow 428$

brevicornis Herrich-Schäffer (Leiophron) $* \rightarrow 832$

brevicornis Herrich-Schäffer (Triaspis) $* \rightarrow 526$

brevicornis Nees (Ephedrus) $\rightarrow 410$

brevicornis Ratzeburg (Meteoras) $\rightarrow 880$

brevicornis Ruthe (Blacus) $\rightarrow 811$

brevicornis Ruthe (Leiophron) $\rightarrow 834$

brevicornis sensu Ruthe (Leiophron) $\rightarrow 835$

brevicotnis Thomson (Chorebus) $* \rightarrow 112$

brevicornis Wesmael (Aleiodes) $\rightarrow 1477$

brevicornis Wesmael (Ascogaster) $* \rightarrow 686$

brevicornis Wesmael (Bracon) ${ }^{*} \rightarrow 558$

brevicornis Wesmael (Cotesia) $* \rightarrow 1068$

brevipalpis auct. (Biosteres) $\rightarrow 1279$

brevipalpis Thomson (Opius) $* \rightarrow 1312$

brevipennis Kieffer (Pambolus) $* \rightarrow$ no German record

brevipes Wesmael (Meteorus) $\rightarrow 857$

brevipes Wesmael misident. (Meteorus) $\rightarrow 849$

brevipesalis Shenefelt (Meteorus) $\rightarrow 849$

brevipetiolatus Thomson (Perilitus) $* \rightarrow 890$

brevis Wesmael (Ichneutes) $* \rightarrow 1015$

breviscapus Thomson (Opius) $\rightarrow 1369$

breviseta Nees (Agathis) $* \rightarrow 12$

brevistigma Tobias (Dacnusa) $* \rightarrow 234$

brevisulcus Thomson (Biosteres) $* \rightarrow 1281$

breviusculus Wesmael (Bracon) ${ }^{*} \rightarrow 559$

breviventris Ratzeburg (Dolichogenidea) $* \rightarrow 1127$

breviventris Szépligeti (Bracon) $\rightarrow 645$

breviventris Thomson (Triaspis) $* \rightarrow 527$

brullaei Lucas (Agathis) $\rightarrow 22$

brunescens Fahringer (Bracon) $* \rightarrow 560$

brunnipes Ruthe (Meteorus) $\rightarrow 849$

brusceanus Fischer (Opius) $\rightarrow 1311$

brutus Papp (Opius) $\rightarrow 1365$

buccatus Thomson (Chelonus) $\rightarrow 718$

bucephala Marshall (Alysia) $\rightarrow 58$

bucephala Papp (Trachyusa) $* \rightarrow$ no German record buhri Griffiths (Chorebus) * $\rightarrow 113$

buolianae Eady \& Clark (Macrocentrus) $* \rightarrow 1023$

butalidis Marshall (Illidops) $* \rightarrow 1182$

caberae Marshall (Protapanteles) $\rightarrow 1263$

caelatus Haliday (Opius) $* \rightarrow 1313$

caenopachoides Ruschka (Dendrosoter) $\rightarrow 748$

caesa Klug (Disophrys) $* \rightarrow 42$

caesia Klug misspell. (Disophrys) $\rightarrow 42$

caesus Haliday (Opius) * $\rightarrow 1314$

caesus Nees (Heterospilus) $* \rightarrow 763$

cajae Bouché (Cotesia) $* \rightarrow 1069$

calcarata sensu Nixon (Microplitis) $\rightarrow 1223$

calcarata Thomson (Microplitis) $\rightarrow 1241$

calcarator Wesmael (Homolobus) $\rightarrow 1014$

calcaratus Ivanov (Dolichogenidea) $\rightarrow 1147$

calceata Haliday (Rasivalva) $* 1269$

calcitrator Curtis (Zele) $\rightarrow 936$

calculator Fabricius (Bassus) $* \rightarrow 26$

californicus Baker (Diaeretiella) $\rightarrow 404$

caligatus Haliday (Zele) $* \rightarrow 937$

callidus auct. (Protapanteles) $\rightarrow 1264$

calliope Nixon (Chorebus) $\rightarrow 123$

callunae Nixon (Cotesia) $\rightarrow 1100$

campestris Starý (Ephedrus) $\rightarrow 412$

camptolomae Watanabe (Meteorus) $\rightarrow 880$

canaliculata Stelfox (Phaenocarpa) $* 329$

canaliculatus Wesmael (Microplitis) $\rightarrow 1231$

candidata Haliday (Dolichogenidea) $* \rightarrow 1128$

canescens Wesmael (Chelonus) $* \rightarrow 704$

canifrons Wesmael (Ascogaster) $* \rightarrow 687$

capeki Nixon (Microplitis) $* \rightarrow 1212$

capeki Taeger (Orgilus) $* \rightarrow 1421$

capito Nees (Diospilus) $* \rightarrow 985$

caraganae Starý (Aphidius) $\rightarrow 377$

carbonaria Wesmael (Deuterixys) $* \rightarrow 1114$

carbonarius Nees (Biosteres) $* \rightarrow 1282$

carbonarius Ratzeburg (Apanteles) $\rightarrow 1057$

carbonarius Szépligeti (Bracon) $\rightarrow 613$

carbonarius Wesmael (Biosteres) $\rightarrow 1293$

carbonator Marshall (Chelonus) $* \rightarrow 705$

cardui Marshall (Lysiphlebus) $\rightarrow 422$

caricicola Mackauer (Praon) $* \rightarrow 454$

caricis Haliday (Monoctonus) * $\rightarrow 433$

caricivorae Fischer (Opius) $* \rightarrow 1315$

carinata Fötster (Tanycarpa) $\rightarrow 364$

carinata Packard (Microgaster) $\rightarrow 1209$

carinata Thomson (Pentapleura) $\rightarrow 325$

carinata Thomson (Psyttalia) $* \rightarrow 1403$

carinaticeps Gahan (Opius) $\rightarrow 1384$

carinator Nees (Aspicolpus) $* \rightarrow 980$

carinatus Hedqvist (Ecphylus) $\rightarrow 759$ 


\begin{tabular}{|c|c|}
\hline carinatus Nees (Eubazus) $* \rightarrow 483$ & chlorophthalmus Spinola (Zele) $* \rightarrow 938$ \\
\hline carinthiaca Fischer (Phaenocarpa) $* \rightarrow 330$ & chromatomyiae Griffiths (Exotela) $* \rightarrow 293$ \\
\hline carissimus Shestakov (Iphiaulax) $\rightarrow 665$ & chrysippe Nixon (Chorebus) $\rightarrow 112$ \\
\hline carpathica van Achterberg (Dapsilarthra) $\rightarrow 274$ & chrysophthalmus Nees (Zele) $\rightarrow 938$ \\
\hline carpatus Say (Apanteles) $* \rightarrow 1042$ & chrysostictus Marshall (Dolichogenidea) $\rightarrow 1156$ \\
\hline carpocapsae Viereck (Ascogaster) $\rightarrow 694$ & ciceris Fischer (Opius) $* \rightarrow 1318$ \\
\hline castaneiventris Thomson (Biosteres) $\rightarrow 1284$ & cinctellus Spinola (Meteorus) $* \rightarrow 854$ \\
\hline castaneum Nees (Dinotrema) $* \rightarrow$ no German record & cinctiventris Fischer (Opius) $* \rightarrow 1319$ \\
\hline castrator Fabricius (Pseudovipio) $* \rightarrow 669$ & cinctus Haliday (Chorebus) $* \rightarrow 115$ \\
\hline catenator Haliday (Colastes) $* \rightarrow 952$ & cingulata Nees (Alysia) $* \rightarrow 51$ \\
\hline caucasica Kokujev (Doryctes) $\rightarrow 754$ & cingulator Nees (Dacnusa) $* \rightarrow 237$ \\
\hline caucasica Tobias (Agathis) $\rightarrow 10$ & cingulator Ratzeburg (Bassus) $\rightarrow 30$ \\
\hline caucasica Tobias (Dolopsidea) $\rightarrow 1443$ & cingulatus Curtis (Coelinidea) $\rightarrow 216$ \\
\hline caucasicus Telenga (Baryproctus) $\rightarrow 547$ & cingulatus Provancher (Ontsira) $\rightarrow 772$ \\
\hline caudalis Hellén (Ontsira) $\rightarrow 771$ & cingulatus Ruthe (Aphidius) $* \rightarrow 378$ \\
\hline caudata Nees (Triaspis) $* \rightarrow 528$ & cingulatus Wesmael (Opius) $* \rightarrow 1320$ \\
\hline caudatulus Thomson (Biosteres) $* \rightarrow 1283$ & cingulipes Nees (Bassus) $* \rightarrow 27$ \\
\hline caudatus Ratzeburg (Bracon) $* \rightarrow 561$ & cingulum Brischke (Macrocentrus) $* \rightarrow 1024$ \\
\hline caudatus Thomson (Microchelonus) $\rightarrow 734$ & circe Haliday (Cratospila) $* \rightarrow 226$ \\
\hline caudatus Thomson (Rhysipolis) $\rightarrow 975$ & circulator Gravenhorst (Coelinius) $\rightarrow 223$ \\
\hline caudatus Wesmael (Utetes) $* \rightarrow 1407$ & circulator Nees (Opius) $* \rightarrow 1321$ \\
\hline caudifer Fischer (Opius) $* \rightarrow 1316$ & circumscriptus Nees (Aleiodes) $* \rightarrow 1456$ \\
\hline caudiger Nees (Bracon) $* \rightarrow 562$ & circumscriptus Nees (Pholetesor) $* \rightarrow 1249$ \\
\hline caudiger Thomson (Bracon) $\rightarrow 645$ & cirsii Curtis (Trioxys) $* \rightarrow 468$ \\
\hline cebes Nixon (Microplitis) $* 1213$ & cirsii Haliday (Aphidius) $\rightarrow 385$ \\
\hline celsus Haliday (Opius) $* \rightarrow 1317$ & cirsii Ivanov? (Aphidius) $\rightarrow 382$ \\
\hline centaureae Griffiths (Dacnusa) $* \rightarrow 235$ & cis Bouché (Meteorus) $* \rightarrow 855$ \\
\hline centaureae Haliday (Misaphidus) $* \rightarrow 429$ & clandestina Haliday (Amyras) $* \rightarrow 66$ \\
\hline centaureae Szépligeti (Bracon) $\rightarrow 595$ & clandestinus Förster (Adelius) $* \rightarrow 1$ \\
\hline cephalotes Haliday (Aphaereta) $* \rightarrow 71$ & claripennis Griffiths (Chorebus) $* \rightarrow 116$ \\
\hline cepheres Nixon misspell. (Dacnusa) $\rightarrow 236$ & claripennis Thomson (Bracon) $* \rightarrow 563$ \\
\hline cephi Rohwer (Heterospilus) $* \rightarrow 764$ & claritibia Papp (Diolcogaster) ${ }^{*} \rightarrow$ record uncertain \\
\hline cerasi Marshall (Monoctonus) $* \rightarrow 434$ & clarus Haliday (Opius) $\rightarrow 1391$ \\
\hline cerasicola Starý (Ephedrus) ${ }^{*} \rightarrow 407$ & clausthalianus Ratzeburg (Bassus) $* \rightarrow 28$ \\
\hline cerealis Curtis (Blacus) $\rightarrow 802$ & claustratus Gautier \& Bonnamour (Cotesia) $\rightarrow 1109$ \\
\hline cerealium Haliday (Perilitus) $* \rightarrow 891$ & clavator Haliday (Rhyssalus) $* \rightarrow 1446$ \\
\hline cerebrator Ratzeburg misspell. (Dolichogenidea) & clavatus Panzer (Spathius) $\rightarrow 783$ \\
\hline$\rightarrow 1128$ & clavicornis Wesmael (Ropalophorus) $* \rightarrow 916$ \\
\hline cerpheres Nixon (Dacnusa) $* \rightarrow 236$ & clavipes Marshall (Acrisis) $* \rightarrow 940$ \\
\hline cerurae Matsumura (Microplitis) $\rightarrow 1233$ & claviventris Ruthe (Leiophron) $\rightarrow 835$ \\
\hline cespidator Thunberg misspell. (Meteorus) $\rightarrow 853$ & claviventris Ruthe (Eubazus) $* \rightarrow 484$ \\
\hline cespitator Thunberg (Meteorus) $* \rightarrow 853$ & claviventris Wesmael (Leiophron) $\rightarrow 840$ \\
\hline chaetoptelii Gautier \& Russo (Ecphylus) $\rightarrow 759$ & claviventris Wesmael (Helcon) $* \rightarrow 1000$ \\
\hline chaitophori Gärdenfors (Ephedrus) $* \rightarrow 408$ & cleoceridis Marshall (Cotesia) $\rightarrow 1068$ \\
\hline chalveri Docavo (Phaenodus) $\rightarrow 972$ & clypealis Thomson (Ascogaster) $\rightarrow 697$ \\
\hline chenopodiaphidis Ashmead (Diaeretiella) $\rightarrow 404$ & clypealis Thomson (Biosteres) $\rightarrow 1292$ \\
\hline chenopodii Förster (Diaeretiella) $\rightarrow 404$ & clypeatus Förster (Neoneurus) $* \rightarrow 883$ \\
\hline chenopodii Griffiths (Chorebus) $* \rightarrow 114$ & coactus Marshall (Leiophron) $\mathrm{V} \rightarrow 842$ \\
\hline hinensis Holmgren (Meteorus) $\rightarrow 876$ & coccinellae Schrank (Dinocampus) $* \rightarrow 828$ \\
\hline lorophthalma auct. (Homolobus) $\rightarrow 1014$ & coccineus Brullé (Iphiaulax) $\rightarrow 664$ \\
\hline hlorophthalmus Haliday (Homolobus) - & colemani Viereck (Aphidius) $* \rightarrow 379$ \\
\hline
\end{tabular}


colesi Drea (Perilitus) $* \rightarrow 892$

collaris Spinola (Macrocentrus) $* \rightarrow 1025$

collaris Telenga (Bracon) $\rightarrow 645$

collaris Thomson (Triaspis) $* \rightarrow 529$

collinus Szépligeti (Bracon) $\rightarrow 645$

colon Haliday (Meteorus) $* \rightarrow 856$

colorativentris Fischer (Biosteres) $\rightarrow 1275$

coloratus Hellén (Aleiodes) $\rightarrow 1473$

colpophorus Wesmael (Bracon) $* \rightarrow 564$

comatus Wesmael (Aulonotus) $* \rightarrow 1274$

combinatus Papp (Microplitis) $* \rightarrow 1214$

comes Nixon (Dacnusa) $\rightarrow 263$

companellae Hartig misspell. (Triaspis) $\rightarrow 530$

compar Marshall (Opius) $* \rightarrow 1322$

compar Ruthe (Blacus) $\rightarrow 795$

complanatus Quilis (Trioxys) $* \rightarrow 469$

complanellae Hartig (Triaspis) $* \rightarrow 530$

compressicornis Ruthe (Trioxys) $* \rightarrow 470$

compressiventre Fischer (Diachasma) $* 1297$

compressiventris Mucsebeck (Glyptapanteles) ${ }^{*} \rightarrow 1161$

compressor Herrich-Schäffer (Petalodes) $* \rightarrow 1484$

comptus Marshall (Bracon) $\rightarrow 644$

concinnum Haliday (Dinotrema) $* \rightarrow 277$

concinnus Ruthe (Adelius) $\rightarrow 2$

concolor Nees (Synaldis) * $\rightarrow 355$

concolor Thomson (Bracon) $\rightarrow 632$

concolorans Marshall (Bracon) $\rightarrow 632$

confinis Ruthe (Meteorus) $\rightarrow 863$

confinis Ruthe (Dacnusa) $* 238$

confinis Szépligeti (Bracon) $\rightarrow 573$

conflucta Fischer (Atopandrium) $\rightarrow 91$

conformis Wesmael (Blacus) $* \rightarrow 794$

confusa Papp (Microgaster) $\rightarrow 1208$

confusus Tremblay \& Eady (Lysiphlebus) $* \rightarrow 420$

congestus Nees (Cotesia) $\rightarrow 1109$

coniferae Haliday (Dolichogenidea) $\rightarrow 1128$

coniferarum Fahringer (Bracon) $* \rightarrow 565$

conjugellae Bengtsson (Bracon) $* \rightarrow 566$

conjugens Nees misspell. (Chaenusa) $\rightarrow 92$

conjungetns Nees (Chaenusa) $* \rightarrow 92$

connexa Nees (Diolcogaster) $* \rightarrow 1117$

consimilis Nees (Meteorus) $* \rightarrow 857$

consobrina Curtis (Ascogaster) $* \rightarrow 688$

consors Fischer (Opius) $\rightarrow 1396$

consors Ruthe (Meteorus) $\rightarrow 863$

conspersae Fiske (Dolichogenidea) $\rightarrow 1145$

conspicuus Wesmael (Bassus) $* \rightarrow 29$

conspurcator Haliday (Phaenocarpa) $* \rightarrow 331$

constrictus Nees (Aphidius) $\rightarrow$ incertae sedis

consuetor Nees (Perilitus) $* \rightarrow 893$

consularis Haliday (Diolcogaster) $\rightarrow 1117$ conterminus Nees (Syntretus) $* \rightarrow 920$

continua Ruthe (Meteorus) $\rightarrow 856$

contracta Haliday (Alloea) $* \rightarrow 48$

contractus Nees (Microchelonus) $* \rightarrow 722$

contractus Ratzeburg (Baeacis) $\rightarrow 983$

contractus Ratzeburg (Ascogaster) $\rightarrow$ incertae sedis

coquilleti Ashmead (Meteorus) $\rightarrow 875$

coracina Thomson (Microplitis) $\rightarrow 1227$

coracinus Thomson (Opius) $* \rightarrow 1323$

corax Marshall (Meteorus) $* \rightarrow 858$

cornelii Haeselbarth (Perilitus) $* \rightarrow 894$

corrugatus Ruthe (Eubazus) $* \rightarrow 485$

corsicus Marshall (Pseudovipio) $* \rightarrow 670$

corvinus Reinhard (Apanteles) $* \rightarrow 1043$

corvulus Marshall (Chelonus) $* \rightarrow 706$

coryphe Nixon (Cotesia) $\rightarrow 1098$

costatus Zetterstedt (Ichneutes) $\rightarrow 1017$

coxalis Nees (Eubazus) $\rightarrow 499$

coxalis Spinola (Aleiodes) $* \rightarrow 1457$

coxator Thomson (Chorebus) $* \rightarrow 117$

crassicalcaratus Viereck (Homolobus) $\rightarrow 1014$

crassicauda Thomson (Bracon) $\rightarrow 597$

crassiceps Thomson (Bracon) $* \rightarrow 567$

crassiceps Thomson (Schizoprymnus) $* \rightarrow 516$

crassicornis Ruthe (Microgaster) $* \rightarrow 1188$

crassicosta Thomson (Dinotrema) $\rightarrow 283$

crassicrus Thomson (Meteorus) $\rightarrow 863$

crassicrus Thomson (Opius) $* \rightarrow 1324$

crassipes Wesmael (Opius) $* \rightarrow 1325$

crassiusculus Szépligeti (Bracon) $\rightarrow 583$

crataegi Ratzeburg (Cotesia) $\rightarrow 1077$

cratospilum Thomson (Orthostigma) $* \rightarrow 314$

crawfordi Rohwer (Diaeretiella) $\rightarrow 404$

creatus Balevski (Cotesia) $\rightarrow 1086$

credne Nixon (Chorebus) $* \rightarrow 118$

credne Nixon (Dolichogenidea) $* \rightarrow 1129$

cremasta Marshall (Wesmaelia) $\rightarrow 935$

crenulatus Thomson (Chorebus) $* \rightarrow 119$

crepidis Haliday (Monoctonus) $* \rightarrow 435$

crithmi Marshall (Aphidius) $\rightarrow 377$

croaticus Quilis (Diaeretiella) $\rightarrow 404$

crocinus Mackauer (Adialytus) $\rightarrow 371$

cruciatus Ratzeburg (Apanteles) $\rightarrow 1044$

crudelis Papp (Dolichogenidea) $\rightarrow 1156$

cruentatus Rondani (Apanteles) $\rightarrow 1044$

cruentatus Ruthe (Eubazus) $* \rightarrow 486$

cruentus Nees (Aleiodes) $* \rightarrow 1458$

csikii Fischer (Opius) $\rightarrow 1338$

cubitalis Fischer (Opius) $* \rightarrow 1326$

cubocephalus Telenga (Chorebus) $* \rightarrow 120$

cultrator Marshall (Cotesia) $\rightarrow 1062$ 
cultus Marshall (Syntretus) $\rightarrow 923$

cunctator Halidas (Clinocentrus) $* \rightarrow 945$

cupidus Gahan (Opius) $\rightarrow 1334$

cuprea Lyle (Cotesia) $* \rightarrow 1070$

cupressobii Starý (Pauesia) $* \rightarrow 440$

curculionum Hartig (Eubazus) $\rightarrow 505$

curtangula Thomson (Alysia) $\rightarrow 62$

curticaudis Szépligeti (Bracon) $\rightarrow 637$

curticaudis Telenga (Macrocentrus) $\rightarrow 1033$

curtipennis Fischer (Orgilus) $\rightarrow 1430$

curtisii Ratzeburg (Dendrosoter) $* \rightarrow 747$

curtungula Thomson (Alysia) $\rightarrow 58$

curvatus Fischer (Opius) $* \rightarrow 1327$

curvicaudis Ratzeburg (Spathius) $* \rightarrow 781$

curvicaudus Mackauer (Trioxys) $* \rightarrow 471$

curvicaudus Ratzeburg misspell. (Spathius) $\rightarrow 781$

curvicrus Thomson (Microgaster) $\rightarrow 1196$

cuspidatus Haliday (Centistes) $* \rightarrow 820$

cybele Nixon (Chorebus) $\rightarrow 121$

cyclogaster Förster (Exotela) $* \rightarrow 294$

cyclops Nixon (Chorebus) $\rightarrow 120$

cylindricus Telenga (Chorebus) $* \rightarrow 121$

cylindricus Wesmael (Helcon) $\rightarrow 999$

cylindrus Nees (Chelonus) $* \rightarrow 707$

cynipum Thomson (Ascogaster) $\rightarrow 694$

cynthius Lyle (Syntretus) $\rightarrow 928$

cyparissa Nixon (Chorebus) $* \rightarrow 122$

cytherea Nixon (Chorebus) $* \rightarrow 123$

dagda Nixon (Chorebus) $* \rightarrow 124$

daimenes Nixon (Chorebus) $* \rightarrow 125$

dallatorrei Szépligeti (Bracon) $\rightarrow 577$

dauci Marshall (Aphidius) $\rightarrow 390$

debilis Förster (Phaenocarpa) $\rightarrow 336$

debilis Wollaston (Perilitus) * $\rightarrow 895$

debilitatum Morley (Atopandrium) $* \rightarrow 91$

decens Tobias (Microplitis) $* \rightarrow 1215$

deceptor Wesmael (Zele) $* \rightarrow 939$

deceptor Wesmael (Perilitus) $* \rightarrow$ no German record

decipiens Prell (Microplitis) $* \rightarrow 1216$

decoloratus Ruthe (Meteorus) $\rightarrow 880$

decora Haliday (Dolichogenidea) $* \rightarrow 1130$

decorator auct. (Rhysipolis) $\rightarrow 977$

decorator Haliday (Rhysipolis) * $\rightarrow 975$

decoratus Hellén (Orgilus) $\rightarrow 1430$

decoris Förster (Gnamptodon) $* \rightarrow 978$

decorus Marshall (Chelonus) $\rightarrow 700$

decrescens Nees (Ademon) $* \rightarrow 1273$

deficiens Ruthe (Leiophron) $* \rightarrow 833$

deflagrator Nees (Cremnops) $\rightarrow 41$

deflagrator Spinola (Cremnops) $\rightarrow 41$

degenerator Marshall (Bracon) $\rightarrow 609$ deione Nixon (Chorebus) $* \rightarrow 126$

delibator Haliday (Bracon) $* \rightarrow 568$

delitutus Papp (Protapanteles) $* \rightarrow 1258$

delphinii Griffiths (Dacnusa) $* \rightarrow 239$

deltiger Haliday (Toxares) $* \rightarrow 465$

delusor Spinola (Bracon) * $\rightarrow 569$

delusor Wesmael (Earinus) $\rightarrow 44$

delusorius Förster (Syrrhizus) $* \rightarrow 934$

dendrolimi Matsumura (Cotesia) $\rightarrow 1090$

dendrolimusi Matsumura (Cotesia) $\rightarrow 1090$

denigrator Fabricius (Iphiaulax) $\rightarrow 665$

denigrator Linné (Atanycolus) $* \rightarrow 541$

dentata Panzer (Phanerotoma) $* \rightarrow 741$

dentator Fabricius (Helconidea) $* \rightarrow 1003$

dentator Nees (Helconidea) $\rightarrow 1004$

dentatus Tobias (Chorebus) $\rightarrow 183$

denticulatus van Achterberg (Eubazus) $\rightarrow 495$

dentifemur Stelfox (Leptotrema) $* 312$

dentifer Thomson (Opius) $\rightarrow 1320$

dentifer Tobias (Ascogaster) $* \rightarrow 689$

depeculator Förster (Opius) $* \rightarrow 1328$

depressa Herrich-Schäffer (Coelinidea) $* \rightarrow 215$

depressiusculus Szépligeti (Bracon) $\rightarrow 597$

deprimator auct. (Microgaster) $\rightarrow 1187$

deprimator Curtis (Hygroplitis) $\rightarrow 1177$

deprimator Fabricius (Microplitis) $* \rightarrow 1217$

deprimator Wesmael (Diolcogaster) $\rightarrow 1115$

desertor Linné (Cremnops) $* \rightarrow 41$

destitutus Ratzeburg (Eubazus) $* 487$

determinatus Förster (Adelius) $* \rightarrow 2$

dice Nixon (Coloneura) * $\rightarrow 224$

dichrocera Königsmann (Idiasta) $* \rightarrow 305$

dichromus Wesmael (Bracon) $* \rightarrow 570$

dictynna Marshall (Dapsilarthra) $* \rightarrow 270$

difficilis Griffiths (Chorebus) $* \rightarrow 127$

difficilis Nees (Cotesia) $\rightarrow 1069$

difficilis Nixon (Aphaereta) $* \rightarrow 72$

dilecta Haliday (Dolichogenidea) $* \rightarrow 1131$

dilutus Ratzeburg (Diolcogaster) $\rightarrow 1117$

dimidiator Nees (Bassus) $* \rightarrow 30$

dimidiatum Thomson (Dinotrema) $* \rightarrow 278$

dimidiatus auct. (Aleiodes) $\rightarrow 1477$

dimidiatus Nees (Bracon) $\rightarrow$ incertae sedis

dimidiatus Wesmael (Hygroplitis) $\rightarrow 1178$

diminuens Nees (Aphidius) $\rightarrow$ incertae sedis

dion Nixon (Illidops) $\rightarrow 1183$

dioryctriae Rohwer (Microtypus) $\rightarrow 1417$

dioryctriae Wilkinson (Dolichogenidea) $\rightarrow 1153$

diremtus Nees (Chorebus) $* \rightarrow 128$

dirona Nixon (Chorebus) $* \rightarrow 129$

discoideus Wesmael (Bracon) $* \rightarrow 571$ 
discolor Förster (Dacnusa) $* \rightarrow 240$

discolor Hellén (Orgilus) $\rightarrow 1430$

discolor Papp (Opius) $\rightarrow 1310$

discolor Wesmael (Homolobus) $* \rightarrow 1011$

dispar Fahringer (Ascogaster) $* \rightarrow 690$

dispar Haliday (Aleiodes) $* \rightarrow 1459$

dispar Nees (Diospilus) $* \rightarrow 986$

dispar Wesmael (Zele) $\rightarrow 936$

disputabilis Kokujev (Doryctes) $\rightarrow 754$

dissector Nees (Aleiodes) $* \rightarrow 1460$

dissimilis Nees (Baeacis) $* \rightarrow 983$

dissimilis Shestakov (Agathis) $\rightarrow 23$

dissolutus Nees (Lysiphlebus) $* \rightarrow 421$

distensor Thunberg (Helcon) $\rightarrow 999$

distinguendus Hertich-Schäffer (Perilitus) $\rightarrow$ incertae sedis

distracta Nees (Synaldis) $* \rightarrow 356$

divergens Bengtsson (Alysia) $* \rightarrow$ no German record

diversiceps Fischer (Alysia) $\rightarrow 55$

diversicotnis Nees (Blacus) $* \rightarrow 795$

diversicornis Wesmael (Aspigonus) $\rightarrow 981$

diversus Szépligeti (Aleiodes) $\rightarrow 1460$

diversus Szépligeti (Opius) $* \rightarrow 1329$

docilis Haliday (Opius) $* \rightarrow 1330$

docilis Nixon (Microplitis) $* \rightarrow 1218$

doderoi Mantero (Monolexis) $\rightarrow 770$

dolens Marshall (Microplitis) $\rightarrow 1239$

donci Marshall (Aphidius) $\rightarrow 390$

dorsale Haliday (Praon) $* \rightarrow 455$

dorsalis Herrich-Schäffer (Aleiodes) $\rightarrow 1458$

dorsalis Nees (Microplitis) $\rightarrow 1229$

dorsalis Spinola (Apanteles) $* \rightarrow 1044$

dorsator Say (Bracon) $\rightarrow 586$

dovnari Tobias (Orgilus) $* \rightarrow 1422$

drusilla Nixon (Dolichogenidea) $* \rightarrow 1132$

dryochares Marshall (Mirax) $\rightarrow 1272$

dubius Förster (Adelius) $* \rightarrow 3$

dubius Marshall (Pambolus) $\rightarrow 970$

dubius Ruthe (Blacus) $\rightarrow 806$

dubius Ruthe (Meteorus) $\rightarrow 879$

dubius Telenga (Aleiodes) $\rightarrow 1480$

dubius Wesmael (Macrocentrus) $\rightarrow 1025$

dubius Wesmael (Perilitus) $* \rightarrow 896$

ductor Thunberg (Aleiodes) $* \rightarrow 1461$

dudichi Papp (Microgaster) $* \rightarrow 1189$

duplobrevicornis Shenefelt (Leiophron) $* \rightarrow 834$

duploclaviventris Shenefelt (Leiophron) $* \rightarrow 835$

dureseaui Fischer (Opius) $* \rightarrow 1331$

dyscritus Quilis (Aphidius) $\rightarrow 385$

ea Nixon (Chorebus) $\rightarrow 112$

eadyi Huddleston (Meteorus) ${ }^{*} \rightarrow 859$ ebeninus Nees (Macrocentrus) $\rightarrow 1025$

ebenius Fahringer (Chelonus) $\rightarrow 707$

eccoptogastri Ratzeburg (Ecphylus) $\rightarrow 759$

edentatus Haliday (Centistes) ${ }^{*} \rightarrow 821$

edentulus Haliday misspell. (Centistes) $\rightarrow 821$

effeminatus Ruthe (Meteorus) $\rightarrow$ incertae sedis

efoveolata Thomson (Aspilota) $* \rightarrow 83$

efoveolatus Thomson (Bracon) $\rightarrow 588$

eglanteriae Haliday (Aphidius) $* \rightarrow 380$

egregia Marshall (Chorebus) $\rightarrow 183$

egregius Kokujev (Ascogaster) $\rightarrow 694$

egregius Schmiedeknecht (Aridelus) $* \rightarrow 791$

elaphus Haliday (Chremylus) $* \rightarrow 944$

elator Fabricius (Earinus) $* \rightarrow 43$

elegans Curtis (Coelinidea) $* \rightarrow 216$

elegans Förster (Rhaconotus) $* \rightarrow 777$

elegans Herrich-Schäffer (Microgaster) $* \rightarrow 1190$

elegans Mackauer (Calaphidius) $* 399$

elegans Nees (Ascogaster) $\rightarrow 696$

elegans Ruthe (Syntretus) * $\rightarrow 921$

elegantula Nixon (Chorebus) $\rightarrow 119$

elongatus Szépligeti (Chelonus) $* \rightarrow 708$

elpis Nixon (Pholetesor) $* \rightarrow 1250$

emacerator Nees (Praon) $\rightarrow$ incertae sedis

emarginata Nees (Dolichogenidea) $* \rightarrow 1133$

emarginatus Herrich-Schäffer (Microchelonus) $\rightarrow 734$

emniscatus Enderlein (Cremnops) $\rightarrow 41$

endymion Griffiths (Chorebus) $* \rightarrow 130$

enephes Nixon (Chorebus) $* \rightarrow 131$

enephes Nixon (Protapanteles) $* \rightarrow 1259$

enervis Nees (Paralipsis) $* \rightarrow 438$

ensiformis Ratzeburg (Dolichogenidea) $* \rightarrow 1134$

ephippium Curtis (Meteorus) $\rightarrow 863$

ephippium Haliday (Diaeretellus) $* \rightarrow 400$

ephippium Nees (Diospilus) $\rightarrow 986$

epinotiae Fischer (Apanteles) $\rightarrow 1058$

epinotiae Watanabe (Ascogaster) $\rightarrow 694$

epinoticida Fischer (Apanteles) $\rightarrow 1058$

epitriphus Marshall misspell. (Bracon) $\rightarrow 572$

epitriptus Marshall (Bracon) * $\rightarrow 572$

equalis Lyle (Macrocentrus) $* \rightarrow 1026$

equestris Haliday (Sathon) $\rightarrow 1271$

equiseticola Starý (Aphidius) $\rightarrow 387$

exasmi Nixon (Dolichogenidea) $* \rightarrow 1135$

eremita Reinhard (Microplitis) $* \rightarrow 1219$

ergias Nixon (Chorebus) * $\rightarrow 132$

eriophori Mackauer (Aphidius) $\rightarrow 382$

erosus Herrich-Schäffer (Microchelonus) $* \rightarrow 723$

errans Nees (Blacus) $* \rightarrow 796$

erraticus Wesmael (Bracon) $* \rightarrow 573$

erro Nixon (Microgaster) $* \rightarrow 1191$ 
ervi Haliday (Aphidius) $* \rightarrow 381$

erythrocephalus Wesmael (Spathius) $* \rightarrow 782$

erythrogaster Abdinbekova (Microplitis) $* \rightarrow 1220$

erythrogaster Förster (Tanycarpa) $\rightarrow 364$

erythrogaster Herrich-Schäffer (Perilitus) $\rightarrow$ incertae sedis

erythrogaster Wesmael (Doryctes) $\rightarrow 754$

erythronotus Förster (Adelius) $* \rightarrow 4$

erythropa Förster (Dinotrema) $* \rightarrow 279$

erythrostictus Marshall (Bracon) $\rightarrow 573$

esbelta Nixon (Chorebus) $* \rightarrow 133$

esenbeckii Curtis (Ascogaster) $\rightarrow 683$

esenbeckii Hartig (Aleiodes) $* \rightarrow 1462$

eucodonis Griffiths (Chorebus) $* \rightarrow 134$

eugenia Haliday (Phaenocarpa) $* \rightarrow 332$

eulipis Nixon (Cotesia) $* \rightarrow 1071$

euphorbiae Bouché (Cotesia) $\rightarrow 1063$

eupolis Nixon (Microgaster) $* \rightarrow 192$

eurinus Telenga (Aleiodes) $* \rightarrow 1463$

europaeica Tobias (Avga) $\rightarrow 943$

evonymellae Bouché (Dolichogenidea) $* 1136$

exannulatus Ratzeburg (Spathius) $\rightarrow 783$

exarator Linné (Spathius) $* \rightarrow 783$

exarator Marshall (Bracon) $* \rightarrow 574$

excerta Thomson misspell. (Utetes) $\rightarrow 1407$

excisa Herrich-Schäffer (Ascogaster) $* \rightarrow 691$

excrucians Haliday (Centistes) $\rightarrow 819$

excubitor Haliday (Clinocentrus) $* \rightarrow 946$

excubitor sensu Telenga (Clinocentrus) $\rightarrow 947$

exhilarator Nees (Bracon) $* \rightarrow 575$

exiguus auct. (Opius) $\rightarrow 1329$

exiguus Haliday (Pholetesor) $\rightarrow 1248$

exiguus Wesmael (Opius) $* \rightarrow 1332$

exilis Förster (Coloneura) $\rightarrow 225$

exilis Haliday (Opius) $\rightarrow 1371$

exilis Haliday (Apanteles) $* \rightarrow$ record uncertain

exilis Marshall (Microchelonus) $* \rightarrow 724$

exilis Nees (Blacus) $* \rightarrow 797$

exilis Reinhard (Apanteles) $\rightarrow 1043$

exoletum Nees misspell. (Praon) $\rightarrow 456$

explorator Szépligeti (Bracon) $\rightarrow 645$

exserens Nees (Dacnusa) $\rightarrow 261$

exsertor Nees (Clinocentrus) $* \rightarrow 947$

exsertor Reinhard (Eubazus) $* \rightarrow 488$

exsertus Fischer (Utetes) $\rightarrow 1415$

exsertus Thomson (Utetes) $\rightarrow 1407$

exsoletum Nees (Praon) $* \rightarrow 456$

extensor Linné (Charmon) $* \rightarrow 679$

extricator Nees (Cyanopterus) $* \rightarrow 660$

fabarum Marshall (Lysiphlebus) $* \rightarrow 422$

facialis Förster (Noserus) $* \rightarrow 969$ facialis Ratzeburg (Triaspis) $* \rightarrow 531$

facialis Ruthe (Meteorus) $\rightarrow 879$

facialis Thomson (Bracon) $\rightarrow 577$

facialis Thomson (Rilipertus) $* \rightarrow 913$

faeroeensis Roman (Dacnusa) $* \rightarrow 241$

falcatus Mackauer (Trioxys) $* \rightarrow 472$

falcatus Nees (Pygostolus) $* \rightarrow 910$

falcatus Nees (Sathon) $* \rightarrow 1271$

falcificum Stelfox \& Graham misspel (Dinotrema) $\rightarrow 280$

falciger Ruthe (Perilitus) $* \rightarrow 897$

falcigera Graham (Aphaereta) $* \rightarrow 73$

falconivibrans Morley (Myiocephalus) $\rightarrow 881$

fallaciosus Szépligeti (Bracon) $\rightarrow 590$

fallax Nixon (Chorebus) $* \rightarrow 135$

fallax Ruthe (Meteorus) $\rightarrow 863$

fallax Szépligeti (Opius) $\rightarrow 1343$

fallax Szépligeti (Opius) $* \rightarrow 1333$

falsificum Stelfox \& Graham (Dinotrema) $* \rightarrow 280$

fasciatae Gautier \& Dresnay (Cotesia) $\rightarrow 1112$

fasciator Spinola (Bracon) $\rightarrow 576$

fasciatorior Shenefelt (Bracon) $* \rightarrow 576$

fasciatus Dahlbom (Ascogaster) $\rightarrow 696$

fasciatus Nees (Eubazus) $* \rightarrow 489$

fasciatus Ratzeburg (Meteorus) $\rightarrow 856$

fascidiator Fabricius misident. (Iphiaulax) $\rightarrow 664$

fasciipennis Ruthe misspell. (Leiophron) $\rightarrow 836$

fascipennis Ruthe (Leiophron) $* \rightarrow 836$

fastidiator Fabricius (Iphiaulax) $* \rightarrow 664$

fausta Nixon (Glyptapanteles) $* \rightarrow 1162$

femoralis Bouché (Dolichogenidea) $\rightarrow 1131$

femoralis Herrich-Schäffer (Ascogaster) $\rightarrow 680$

femoralis Thomson (Helcon) $\rightarrow 1001$

femorator Tobias (Cenocoelius) $\rightarrow 678$

fenestratus Nees (Chelonus) $\rightarrow 700$

fenestratus Nees (Microchelonus) $* \rightarrow 725$

ferga Papp (Phaenocarpa) $\rightarrow 344$

ferruginea Marshall (Cotesia) $* \rightarrow 1072$

flator Haliday (Meteorus) $* \rightarrow 860$

filator Nees (Diospilus) $\rightarrow 986$

filicornis Haeselbarth (Blacus) $* \rightarrow 798$

filicornis Thomson (Opius) $\rightarrow 1350$

filiformis Ratzeburg (Coeloides) $* \rightarrow 652$

firmus Ratzeburg (Eubazus) $\rightarrow 502$

firmus Telenga (Apanteles) $\rightarrow 1049$

fischeri Haeselbarth (Blacus) $* \rightarrow 799$

fissuralis Tobias (Microchelonus) $\rightarrow 735$

fissus Jakimavicius (Biosteres) $\rightarrow 1291$

flagellatis Thomson (Bracon) $* \rightarrow 577$

flagellatus Alexeev (Meteorus) $\rightarrow 857$

flagitator Curtis (Homolobus) $* \rightarrow 1012$ 
flavator Fabricius (Cyanopterus) $* \rightarrow 661$ flaveolus Györfi (Toxares) $\rightarrow 465$ flavescens Fahringer (Phanerotoma) $\rightarrow 741$ flaviceps Marshall (Streblocera) $* \rightarrow 917$ flavicornis Nees (Aspigonus) $* \rightarrow 981$ flavicoxa Thomson (Antrusa) $* \rightarrow 68$ flavicoxa Thomson misspell. (Rhysipolis) $\rightarrow 977$ flavidens Ratzeburg (Aphidius) $\rightarrow$ incertae sedis flavifrons Haliday (Cenocoelius) $\rightarrow 677$ flavilabris Ratzeburg (Apanteles) $\rightarrow 1054$ flavinode Haliday (Praon) $* \rightarrow 457$ flavipalpis Brullé (Microplitis) $* \rightarrow 1221$ flavipalpis Wesmael (Triaspis) $* \rightarrow 532$ flavipes Förster (Phaenodus) $\rightarrow 972$ flavipes Goureau (Chorebus) $* \rightarrow 136$ flavipes Haliday (Diolcogaster) $* \rightarrow 1118$ flavipes Haliday (Eubazus) $* \rightarrow 490$ flavipes Haliday (Phaenocarpa) $* \rightarrow 333$ flavipes Ivanov (Triaspis) $\rightarrow 530$ flavipes Nees (Bracon) $* \rightarrow 578$ flavipes Ratzeburg (Macrocentrus) $\rightarrow 1036$ flavipes Šnoflak (Phanerotomella) $\rightarrow 744$ flavitarsis Thomson (Colastes) $* \rightarrow 953$ flaviventris Förster (Dendrosoter) $\rightarrow 748$ flaviventris Haliday (Dapsilarthra) $\rightarrow 272$ flaviventris Thomson (Colastes) $\rightarrow 959$ flavolimbatus Ratzeburg (Pholetesor) $\rightarrow 1249$ flavonode Haliday misspell. (Praon) $\rightarrow 457$ flavulator Ratzeburg (Cyanopterus) $\rightarrow 661$ flavus Snellen van Vollenhoven (Macrocentrus) * $\rightarrow 1027$

flavus Tobias (Adelius) $\rightarrow 4$

flexuosus Herrich-Schäffer (Coelinius) $\rightarrow 223$

floricola Wesmael (Triaspis) $* \rightarrow 533$

florimela Haliday (Adelurola) $* \rightarrow 46$ fodori Papp (Meteorus) $\rightarrow 869$

foersteri Haeselbarth misspell. (Coeloides) $\rightarrow 653$ foersteri Marshall (Monolexis) $* \rightarrow 769$

foersteri Shenefelt (Tanycarpa) $\rightarrow 364$ foersteri Tobias (Microchelonus) ${ }^{*} \rightarrow$ record uncertain fordi Nixon (Chorebus) $* \rightarrow 137$

fordi Nixon (Mictoplitis) $* \rightarrow 1222$

formicaria Goidanich (Protaphidius) $\rightarrow 464$ formosus Giraud (Aleiodes) $\rightarrow 1467$ formosus Wesmael (Meteorus) $\rightarrow 869$ formosus Wesmael (Protapanteles) $* \rightarrow 1260$ forsteri Haeselbarth (Coeloides) $* \rightarrow 653$ fortipes Reinhard (Bassus) $* \rightarrow 31$ fortipes Wesmael (Bracon) $* \rightarrow 579$ fossulatus Bouché (Microplitis) $\rightarrow 1236$ foveola Thomson (Bracon) $\rightarrow 573$ foveolator Thomson (Colastes) $* \rightarrow 954$ foveolatus Herrich-Schäffer (Polydegmon) $* \rightarrow 511$ foveolatus Reinhard (Perilitus) $* \rightarrow 898$ foveolus Haliday (Chorebus) $* \rightarrow 138$ fragilis Haliday (Meteorus) $\rightarrow 856$ fragilis Haliday (Colastes) $* \rightarrow 955$ fragilis Wesmael (Meteorus) $\rightarrow 856$ fraternus Reinhard (Glyptapanteles) $* \rightarrow 1163$ fraudator Marshall (Bracon) $\rightarrow 548$ frigida Haliday (Alysia) $* \rightarrow 52$ fritzmuelleri Mackauer (Lysiphlebus) $* \rightarrow 423$ fucicola Haliday (Syncrasis) $* \rightarrow 358$ fulcriger Wesmael (Glyptapanteles) $\rightarrow 1176$ fulgidum Haliday (Diachasma) $* \rightarrow 1298$ fuliginosa Haliday (Pentapleura) $* \rightarrow 325$ fuliginosus Wesmael (Pholetesor) $\rightarrow 1255$ fulviceps Kriechbaumer (Atanycolus) $* \rightarrow 542$ fulviceps Ruthe (Perilitus) $* \rightarrow 899$ fulviceps Westwood (Streblocera) $* \rightarrow 918$ fulvicollis Thomson (Opius) $* \rightarrow 1334$ fulvicornis Wesmael (Microplitis) $* \rightarrow 1223$ fulvicrus Thomson (Microgaster) $* \rightarrow 1193$ fulvipes Haliday (Triaspis) $\rightarrow 536$ fulvipes Haliday (Glyptapanteles) $* \rightarrow 1164$ fulvipes Howard \& Fiske (Glyptapanteles) $\rightarrow 1167$ fulvipes Nees (Bracon) $* \rightarrow 580$ fulviventris Curtis (Ascogaster) $\rightarrow 680$ fulvus Szépligeti (Bracon) $\rightarrow 590$ fumariae Quilis (Misaphidus) $\rightarrow 427$ fumatus Herrich-Schäffer (Schizoprymnus) $* \rightarrow 517$ fumigatus Szépligeti (Bracon) $\rightarrow 590$ fumigidus Szépligeti (Bracon) $\rightarrow 606$ fumipennis Györfi (Aphidius) $\rightarrow 381$ fumipennis Ratzeburg (Microplitis) $\rightarrow 1240$ fumipennis Thomson (Bracon) $\rightarrow 614$ funebris Mackauer (Aphidius) $* \rightarrow 382$ funebris Wesmael (Opius) $\rightarrow 1379$ funestus Haliday (Pseudobathystomus) ${ }^{*} \rightarrow 1445$ fuscatus Nees (Doryctes) ${ }^{*} \rightarrow 751$ fuscicornis Cameron (Apanteles) $\rightarrow 1042$ fuscicornis Förster (Monolexis) $* \rightarrow 770$ fuscicornis Haliday (Aspilota) $* \rightarrow 84$ fuscicoxis van Achterberg \& Haeselbarth (Syntretus) $* \rightarrow 922$

fuscicoxis Wesmael (Bracon) $* 581$ fuscipalpis Wesmael (Eubazus) $\rightarrow 489$ fuscipennis Haliday (Alysia) $* 53$ fuscipennis Nixon (Chorebus) $* \rightarrow 139$ fuscipennis Thomson (Ascogaster) $\rightarrow 685$ fuscipennis Thomson (Bracon) $\rightarrow 614$ fuscipennis Wesmael (Bracon) $* \rightarrow 582$ 
fuscipennis Wesmael (Opius) $* \rightarrow 1335$

fuscipennis Zetterstedt (Agathis) $* \rightarrow 13$

fuscipes Haliday (Syncrasis) $\rightarrow 359$

fuscipes Hellén (Acrisis) $* 941$

fuscipes Herrich-Schäffer (Eubazus) $* \rightarrow 491$

fuscipes Nees (Aphaereta) $\rightarrow 75$

fuscipes Nees (Centistes) $* \rightarrow 822$

fuscipes Wesmael (Meteotus) $\rightarrow 854$

fuscitarsis Bengtsson (Homolobus) $\rightarrow 1014$

fusciventris Hellén (Diospilus) $* \rightarrow 987$

fuscula Haliday (Chorebus) $\rightarrow 152$

gabrielis Gautier \& Riel (Cotesia) $\rightarrow 1085$

gades Nixon (Cotesia) $* \rightarrow 1073$

gagates Nees (Dolichogenidea) $* \rightarrow 1137$

galatea Haliday (Phaenocarpa) $* \rightarrow 334$

galba Nixon (Chorebus) $\rightarrow 189$

galii Griffiths (Chorebus) $* \rightarrow 140$

gallarum Ratzeburg (Bracon) $\rightarrow 618$

gallarum Ratzeburg (Dacnusa) $* \rightarrow$ no German record

gallicum Starý (Praon) * $\rightarrow 458$

gallicus Reinhard (Ontsira) $\rightarrow 771$

gallicus Reinhard (Eubazus) $* \rightarrow 492$

gallicus Thomson (Bracon) $\rightarrow 583$

ganesa Nixon (Chorebus) $* \rightarrow 141$

gasterator auct. (Aleiodes) $\rightarrow 1477$

gastropachae Bouché (Cotesia) $* \rightarrow 1074$

gedanensis Ratzeburg (Chorebus) $* \rightarrow 142$

geminator Lyle (Homolobus) $\rightarrow 1012$

genalis Thomson (Atanycolus) $* \rightarrow 543$

geniculator Nees (Aleiodes) $\rightarrow 1480$

geniculatus Thomson (Opius) $* \rightarrow 1336$

genistae Mackauer (Misaphidus) $* \rightarrow 430$

gentianae Griffiths (Dacnusa) $* \rightarrow 242$

gentianellus Griffiths (Chorebus) * $\rightarrow 143$

genualis Marshall (Agathis) $\rightarrow 21$

germanica Enderlein (Bassus) $\rightarrow 36$

germanicus Belokobylskij (Doryctes) $* \rightarrow 752$

germanus Haliday (Adelius) $* \rightarrow 5$

geryonis Marshall (Cotesia) $* \rightarrow 1075$

gibber Eady \& Clark (Macrocentrus) $* \rightarrow 1028$

gibbiscuta Thomson (Ascogaster) $\rightarrow 685$

gifuensis Ashmead (Macrocentrus) $\rightarrow 1031$

gifuensis auct. (Macrocentrus) $\rightarrow 1024$

gigapiceus Fischer (Opius) $* \rightarrow 1337$

gigas Tobias (Rhysipolis) $\rightarrow 977$

gigas Wesmael (Pygostolus) $\rightarrow 912$

gilvus Papp (Agathis) $\rightarrow 20$

girkanus Tobias? (Pholetesor) $\rightarrow 1250$

glaber Nixon (Chorebus) $* \rightarrow 144$

glabrata Fahringer (Eubazus) $\rightarrow 505$ glabtata Telenga (Cotesia) $* \rightarrow 1076$

glabratus Ceballos (Nicrogaster) $\rightarrow 1194$

glabricollis Telenga (Agathis) $\rightarrow 23$

glabricollis Thomson (Colastes) $\rightarrow 950$

glabricula Thomson (Agathis) $\rightarrow 13$

glabriculus Thomson (Chorebus) $* \rightarrow 145$

gladiator Szépligeti (Sathon) $\rightarrow 1271$

globata Linné (Microgaster) $* \rightarrow 1194$

globatus Bouché (Cotesia) $\rightarrow 1109$

globatus Panzer (Cotesia) $\rightarrow 1063$

globosus Szépligeti (Schizoprymnus) $\rightarrow 524$

glomerata Linné (Cotesia) $* \rightarrow 1077$

glomeratus sensu Curtis (Cotesia) $\rightarrow 1098$

glomeratus sensu Nees (Glyptapanteles) $\rightarrow 1164$

glomeratus sensu Newman (Cotesia) $\rightarrow 1069$

gloratorius Panzer misspell. (Eatinus) $\rightarrow 44$

gloriator Nees (Earinus) $\rightarrow 44$

gloriatorius Panzer (Earinus) $* \rightarrow 44$

gnaphalii Griffiths (Chorebus) $* \rightarrow 146$

goidanichi Quilis (Aphidius) $\rightarrow 385$

gomezi Quilis (Lysiphlebus) $\rightarrow 422$

gonopterygis Marshall (Cotesia) $* \rightarrow 1078$

gossypinus Retzius (Microgaster) $\rightarrow 1194$

gracilariae Wilkinson (Dolichogenidea) $* \rightarrow 1138$

gracilicornis Förster (Acrisis) $* \rightarrow 942$

gracilicornis Nees (Tanycarpa) $* \rightarrow 363$

gracilipes Telenga (Macrocentrus) $\rightarrow 1037$

gracilipes Thomson (Clinocentrus) $\rightarrow 945$

gracilipes Thomson (Cotesia) $\rightarrow 1109$

gracilis Curtis (Coelinidea) $* \rightarrow 217$

gracilis Fischer (Opius) $* \rightarrow 1338$

gracilis Förster (Lipolexis) $* \rightarrow 417$

gracilis Herrich-Schäffer (Triaspis) $\rightarrow 528$

gracilis Marshall (Deuterixys) $\rightarrow 1114$

gracilis Nees (Chorebus) $\rightarrow 183$

gracilis Provancher (Charmon) $\rightarrow 679$

gracilis Ruthe (Meteorus) $\rightarrow 851$

gracilis Ruthe (Microplitis) $\rightarrow 1238$

graeffei Fischer (Heterospilus) $\rightarrow 767$

graeffei Fischer (Meteorus) $\rightarrow 874$

grahami Huddleston (Ascogaster) $* \rightarrow 692$

granarius Marshall (Aphidius) $\rightarrow 377$

granatensis Quilis (Misaphidus) $\rightarrow 427$

grandiceps Thomson (Bracon) $* \rightarrow 583$

grandiceps Thomson (Leiophron) $* \rightarrow 837$

grandii Goidanich (Macrocentrus) $\rightarrow 1024$

grandis Giraud (Aleiodes) $\rightarrow 1453$

grandis Thomson (Microgaster) $\rightarrow 1197$

gravenhorsti Wesmael (Microchelonus) $\rightarrow 726$

gravenhorstii Nees (Microchelonus) $* \rightarrow 726$

gravis Herrich-Schäffer (Coelinidea) $* \rightarrow 218$ 
gregarius Marshall (Aphidius) $\rightarrow 378$ gregori Šnoflak (Phanerotoma) $\rightarrow 740$ griseifrons Thomson (Agathis) $* \rightarrow 14$ groschkeana Griffiths (Dacnusa) $* \rightarrow 243$ groschkei Griffiths (Chorebus) $* \rightarrow 147$ grossum Starý (Praon) $\rightarrow 455$ grunini Tobias (Orgilus) $* \rightarrow 1423$ gussakovskyi Tobias (Agathis) $\rightarrow 22$ guttator Panzer (Bracon) $* \rightarrow 584$ guttiget Wesmael (Bracon) $* \rightarrow 585$ guttiventris Thomson (Pseudovipio) $* \rightarrow 671$ gytator Thunberg (Meteorus) $\rightarrow 873$ hades Papp (Bracon) $\rightarrow 573$ haemorrhoeus Haliday (Biosteres) $* \rightarrow 1284$ haeselbarthi van Achterberg (Apronopa) $* \rightarrow 78$ halidaii Marshall (Neoneurus) $\rightarrow 882$ halidayi Fahringer (Microplitis) $\rightarrow 1229$ halidayi Förster (Syncrasis) $* \rightarrow 359$ hariolator Haliday (Rhysipolis) $* \rightarrow 976$ harpyae Niezabitowski (Cotesia) $\rightarrow 1063$ hartigi Ratzeburg misspell. (Dendrosoter) $\rightarrow 748$ hartigi Shenefelt (Meteorus) $\rightarrow 880$ hartigii Ratzeburg (Dendrosoter) $* \rightarrow 748$ hastatus Haliday (Blacus) $* \rightarrow 800$ hastifer Marshall (Dolopsidea) $\rightarrow 1443$ hawaiiensis Ashmead (Apanteles) $\rightarrow 1042$ hebetor Say (Bracon) $* \rightarrow 586$ hedymeles Nixon (Apanteles) $\rightarrow 1056$ heinzei Mackauer (Diaeretellus) $* \rightarrow 401$ heinzi Mackauer misspell. (Diaeretellus) $\rightarrow 401$ heliophilus Fischer (Meteorus) $* \rightarrow 861$ helleni Nixon (Dolichogenidea) $* \rightarrow 1139$ hemiflavus Szépligeti (Bracon) $\rightarrow 645$ hemipterus Thomson (Heterospilus) $* \rightarrow 765$ hemirugosus Szépligeti (Bracon) $\rightarrow 590$ hera Nixon (Exotela) $* \rightarrow 295$ heraclei Haliday (Misaphidus) $* \rightarrow 431$ heringi Fischer (Opius) $* \rightarrow 1339$ heringi Griffiths (Dacnusa) $* \rightarrow 244$ heringianus Griffiths (Chorebus) $* \rightarrow 148$ heterocera Thomson (Tates) $* \rightarrow 365$ heterocerus Ruthe (Microplitis) $* \rightarrow 1224$ heterogaster Wesmael (Aleiodes) $\rightarrow 1448$ heteroneurus Thomson (Meteorus) $\rightarrow 875$ heterotergis Fahringer (Cotesia) $\rightarrow 1077$ heydeni Reinhard misspell. (Doryctes) $\rightarrow 753$ heydenii Reinhard (Doryctes) $* \rightarrow 753$ hians Nees (Trachionus) $* \rightarrow 366$ hians Stelfox (Eurytenes) $\rightarrow 1302$ hiator Thunberg (Taphaeus) $* \rightarrow 1005$ hieraciorum Starý (Aphidius) $* \rightarrow 383$ hilaris Fischer (Utetes) $\rightarrow 1409$

hilaris Herrich-Schäffer (Schizoprymnus) $* \rightarrow 518$

hinzi Nixon (Diolcogaster) $* \rightarrow 1119$

hitsutipes Huddleston (Meteorus) ${ }^{*} \rightarrow 862$

hirticornis Mackauer (Lysiphlebus) $* \rightarrow 424$

hirticornis Thomson (Aspilota) $* \rightarrow 85$

hirtus Thomson (Aleiodes) $\rightarrow 1473$

hodisensis Fischer (Meteorus) $\rightarrow 860$

holconotus Fischer (Opius) $* \rightarrow 1340$

holmani Starý (Ephedrus) $\rightarrow 413$

homostigma Fahringer (Ephedrus) $\rightarrow 409$

hoplites Ratzeburg (Dolichogenidea) $\rightarrow 1147$

hortensis Marshall (Aphidius) $* \rightarrow 384$

hortorum Starý (Trioxys) $* \rightarrow 473$

hospes Marshall (Microgaster) $* \rightarrow 1195$

hospita Förster (Dacnusa) $* 245$

hostilis Haeselbarth (Blacus) $* \rightarrow 801$

hostium Fischer (Utetes) $\rightarrow 1409$

humeralis Zetterstedt (Meteorus) $\rightarrow 853$

humilis Nees (Blacus) $* \rightarrow 802$

humilis Thomson (Chelonus) $\rightarrow 718$

humuli Mackauer (Trioxys) $* \rightarrow 474$

hungaricus Györfi (Aphidius) $\rightarrow 377$

hungaricus Szépligeti (Baryproctus) $\rightarrow 547$

hungaricus Szépligeti (Opius) $\rightarrow 1365$

hungaricus Szépligeti (Colastes) ${ }^{*} \rightarrow 956$

hyalinipennis Förster (Adelius) $* \rightarrow 6$

hyalipennis Förster (Adelius) $\rightarrow 6$

hydrelliae Rimsky-Korsakov (Opius) $\rightarrow 1314$

hylesini Ratzeburg (Ecphylus) $\rightarrow 759$

hylobii Ratzeburg (Bracon) $* \rightarrow 587$

hyosciamellus Viereck (Biosteres) $\rightarrow 1291$

hyperboreus Hellén (Orgilus) $\rightarrow 1426$

hyphantriac Riley (Cotesia) $* \rightarrow 1079$

iapetus Nixon (Protapanteles) $* \rightarrow 1261$

iarbas Nixon (Dolichogenidea) $\rightarrow 1136$

ictericus Nees (Meteorus) $* \rightarrow 863$

icterius Nees misspell. (Meteorus) $\rightarrow 863$

idalius Haliday (Syntretus) $* \rightarrow 923$

idia Nixon (Microplitis) $* \rightarrow 1225$

igae Watanabe (Apanteles) $\rightarrow 1042$

ignea Ratzeburg (Ontsira) $\rightarrow 772$

ikonomovi Fischer (Meteorus) $\rightarrow 880$

ilicis Nixon (Opius) $\rightarrow 1377$

ilinskyi Glowacki \& Karpinski (Microtypus) $\rightarrow 1416$

imminens Marshall (Pambolus) $\rightarrow 970$

immissa Papp (Dolichogenidea) $* \rightarrow 1140$

immunis Haliday (Protapanteles) $* \rightarrow 1262$

immutator Nees (Bracon) $* \rightarrow 588$

impatientis Fischer (Opius) $* \rightarrow 1341$

impavidus Gautier \& Dresnay (Glyptapanteles) $\rightarrow 1176$ 
imperator Haliday (Ontsita) $* 772$

imperator Wilkinson (Dolichogenidea) $* \rightarrow 1141$

imperialis A. Costa (Disophrys) $\rightarrow 42$

impostor Scopoli (Iphiaulax) $* \rightarrow 665$

impressus Granger (Ephedrus) $\rightarrow 413$

impressus Herrich-Schäffer (Ascogaster) $\rightarrow 694$

impressus Mackauer (Aphidius) $\rightarrow 396$

impressus Wesmael (Biosteres) $\rightarrow 1282$

impressus Wesmael (Microplitis) * $\rightarrow 1226$

impura Nees (Dolichogenidea) $* \rightarrow 1142$

inanitus Linné (Chelonus) $* \rightarrow 709$

incertoides Telenga (Aleiodes) $\rightarrow 1454$

incertus Ashmead (Ontsira) $\rightarrow 771$

incertus Fischer misspell. (Utetes) $\rightarrow 1415$

incertus Kokujev (Aleiodes) $\rightarrow 1454$

incertus Ruthe (Protapanteles) $* 1263$

incertus Wesmael (Colastes) $* \rightarrow 957$

incidens Thomson (Dacnusa) $\rightarrow 227$

incisulus Fischer (Opius) $* \rightarrow 1342$

inclusus Ratzeburg (Glyptapanteles) $* 1165$

incompletus auct. (Heterospilus) $\rightarrow 762$

incompletus Ratzeburg (Caenophanes) $* \rightarrow 746$

incompletus Thomson (Dendrosoter) $\rightarrow 750$

incongrua Nees (Alysia) $* \rightarrow 54$

inculcator auct. (Disophrys) $\rightarrow 42$

inculcator Linné misident. (Disophrys) $\rightarrow 42$

indagator Haliday (Dolopsidea) $* \rightarrow 1443$

indagatrix Förster (Mesocrina) $* \rightarrow 313$

indivisus Quilis (Aphidius) $\rightarrow 387$

indotatus Viereck (Biosteres) $\rightarrow 1287$

indubius Szépligeti (Bracon) $\rightarrow 606$

inermis Quilis (Lysiphlebus) $\rightarrow 422$

infima Haliday (Dolichogenidea) $* \rightarrow 1143$

infirmus Leonardi (Eubazus) $\rightarrow 502$

infirmus Nees (Aphidius) $\rightarrow 381$

infirmus Nees (Macrocentrus) $* \rightarrow 1029$

inflexus Reinhard (Diospilus) $* \rightarrow 988$

infulata Haliday (Pauesia) $* \rightarrow 441$

infumator Lyle (Homolobus) $* \rightarrow 1013$

infumatus Haliday (Hygroplitis) $\rightarrow 1177$

infuscatus Hellén (Aleiodes) $\rightarrow 1477$

ingenuoides Papp (Apanteles) $* \rightarrow 1045$

ingratus Haliday (Mictoplitis) $\rightarrow 1217$

initiator auct. (Atanycolus) $\rightarrow 543$

initiator Fabricius (Atanycolus)* $\rightarrow$ record uncertain

initiator Fonscolombe (Disophrys) $\rightarrow 42$

innovatus Quilis (Lysiphlebus) $\rightarrow 422$

inops Förster (Panerema) * $\rightarrow 323$

inscriptor Nees (Pseudovipio) $* \rightarrow 672$

insertus Fischer (Utetes) $\rightarrow 1415$

insidens Ratzeburg (Cotesia) $\rightarrow 1105$ insignis Förster (Dendrosoter) $\rightarrow 750$

insignis Muesebeck (Meteorus) $\rightarrow 878$

instabilis Marshall (Bracon) $* \rightarrow 589$

instabilis Ruthe (Blacus) $* \rightarrow 803$

instabilis Wesmael (Ascogaster) $\rightarrow 680$

instabilis Wesmael (Opius) $* 1343$

insularis Snellen van Vollenhoven (Agathis) $\rightarrow 20$

intactus Haliday (Leiophron) $\rightarrow 840$

integer von Block (Chelonus) $\rightarrow 712$

intercessor Nees (Bracon) $* \rightarrow 590$

interjectus Taeger (Orgilus) $* \rightarrow 1424$

intermedia Förster (Baeacis) $* \rightarrow 984$

intermedia Ivanov (Microgaster) $\rightarrow 1206$

intermedius Ivanov (Bassus) $\rightarrow 39$

intermedius Szépligeti (Polydegmon) $\rightarrow 511$

intermedius Thomson (Chelonus) $\rightarrow 711$

intermedius Wesmael (Rhysipolis) $\rightarrow 977$

interstitialis Ratzeburg (Macrocentrus) $\rightarrow 1036$

interstitialis Ratzeburg (Eubazus) $* \rightarrow 493$

interstitialis Ruthe (Blacus) $* \rightarrow 804$

interstitialis Thomson (Chorebus) $* \rightarrow 149$

interstitialis Watanabe (Ephedrus) $\rightarrow 413$

intricator Ratzeburg (Macrocentrus) $\rightarrow 1036$

intricatus Haliday (Cotesia) $\rightarrow 1109$

intricatus Ruthe (Rilipertus) $* \rightarrow 914$

iridescens French (Macrocentrus) $\rightarrow 1031$

irtegularis Wesmael (Aleiodes) $\rightarrow 1482$

irregularis Wesmael (Opius) $* \rightarrow 1344$

irrorator Fabricius (Sigalphus) $* \rightarrow 1488$

isabella Haliday (Dapsilarthra) $* \rightarrow 271$

ischnus Marshall (Orgilus) $* 1425$

islandicus Ruthe (Meteorus) $\rightarrow 875$

isolde Nixon (Cotesia) $* \rightarrow 1080$

isomera Förster (Opius) $\rightarrow 1313$

itea Nixon (Dolichogenidea) $\rightarrow 1155$

iuxtangelum Fischer (Opius) $* \rightarrow 1345$

ivanovi Kokujev (Atanycolus) $\rightarrow 546$

ivanowi Kokujev misspell. (Atanycolus) $\rightarrow 546$

iziphyae Mackauer (Trioxys) $* \rightarrow 475$

jaculator Haliday (Meteorus) $* \rightarrow 864$

janinii Quilis (Lysiphlebus) $\rightarrow 422$

japonica Watanabe (Homolobus) $\rightarrow 1013$

japonicus Ashmead (Chremylus) $\rightarrow 944$

japonicus Ashmead (Ephedrus) $\rightarrow 414$

japonicus Ashmead (Glyptapanteles) $\rightarrow 1167$

japonicus Ashmead (Meteorus) $\rightarrow 874$

jaroshevskyi Telenga (Clinocentrus) $\rightarrow 947$

jucunda Marshall (Cotesia) $* \rightarrow 1081$

juglandis Ashmead (Bracon) $\rightarrow 586$

jugosus Lyle (Protapanteles) $\rightarrow 1263$

juniperatae Bouché (Cotesia) $* \rightarrow 1082$ 
juniperorum Starý (Pauesia) $* \rightarrow 442$

kacheticus Telenga (Bracon) $\rightarrow 590$

kama Nixon (Chorebus) $* \rightarrow 150$

kamtshaticus Tobias (Microchelonus) $\rightarrow 720$

karesuandensis Fischer (Opius) $* 1346$

kasachstanica Tobias (Agathis) $\rightarrow 17$

kaszabi Papp (Bassus) $\rightarrow 32$

katkowi Kokujev (Cardiochiles) $\rightarrow 676$

kiritshenkoi Telenga (Bracon) $\rightarrow 580$

klemensiewiczii Niezabitowski (Gnamptodon) $\rightarrow 978$

kJugii Nees (Ascogaster) $* \rightarrow 693$

klugii Ratzeburg (Cosmophorus) $* \rightarrow 827$

klugii Ruthe (Syntretus) $* \rightarrow 924$

knautiae Griffiths (Chorebus) $* \rightarrow 151$

kokujevi Tobias (Diospilus) $* \rightarrow 989$

kolazyi Fischer (Agathis) $\rightarrow 22$

kolthoffi Fahringer (Aleiodes) $\rightarrow 1457$

konovii Marshall (Cyanopterus) $* \rightarrow 662$

konowii Marshall misspell. (Cyanopterus) $\rightarrow 662$

kudsiricus Papp (Bracon) $\rightarrow 557$

kurdjumovi Telenga (Cotesia) $* \rightarrow 1083$

kurnakovi Tobias (Macrocentrus) $* \rightarrow 1030$

kuslitzkyi Tobias (Aleiodes) $\rightarrow 1479$

labilis Ruthe (Perilitus) $* \rightarrow 900$

labrator Ratzeburg (Bracon) $* \rightarrow 591$

lacertosus Haliday (Ephedrus) $* \rightarrow 409$

lactea Nees (Dolichogenidea) $* \rightarrow 1144$

lacteicolor Viereck (Dolichogenidea) $* \rightarrow 1145$

lacteipennis Curtis (Dolichogenidea) $* \rightarrow 1146$

lacteoides Nixon (Iconella) $* \rightarrow 1180$

lactipennis Ratzeburg? (Dolichogenidea) $\rightarrow 1157$

laeta Nixon (Dacnusa) $* \rightarrow 246$

lactatorius Fischer (Opius) $* \rightarrow 1347$

laetus Marshall (Pholetesor) $* \rightarrow 1251$

laetus Wesmael (Bracon) $* \rightarrow 592$

laevicollis Thomson (Ephedrus) $* \rightarrow 410$

laevigata Ratzeburg (Dolichogenidea) $* \rightarrow 1147$

laevigatissimus Dalla Torre (Bracon) ${ }^{*} \rightarrow 593$

laevigatoides Nixon (Dolichogenidea) $* \rightarrow 1148$

laevigator Nees (Orgilus) $\rightarrow$ incertae sedis

laevigator Ratzeburg (Ascogaster) $\rightarrow 695$

laevigatus Förster (Opius) $* \rightarrow 1348$

laevigatus Förster (Rhinoplus) $* \rightarrow 1404$

laevigatus Herrich-Schäffer (Aleiodes) $* \rightarrow 1464$

laevigatus Ratzeburg (Bracon) $\rightarrow 593$

laevigatus Ratzeburg (Oncophanes) $\rightarrow 1444$

laevipectus Thomson (Dacnusa) $* \rightarrow 247$

laevipleuris Tobias (Pentapleura) $\rightarrow 324$

laevis Herrich-Schäffer (Eubazus) $\rightarrow 490$

laevis Thomson (Colastes) $* \rightarrow 958$

laeviscuta Thomson (Microgaster) $\rightarrow 1194$ laevissimus Ratzeburg (Dolichogenidea) $* \rightarrow 149$

laeviventris Ruthe (Leiophron) $\rightarrow 830$

laeviventris Wesmael misspell. (Meteorus) $\rightarrow 875$

lagodechianus Telenga (Bracon) $\rightarrow 573$

lambersi Mackauer (Trioxys) $* \rightarrow 476$

lancearius Ruthe (Perilitus) $* \rightarrow 901$

lanceolator Nees (Oncophanes) $\rightarrow 1444$

lanceolatus Herrich-Schäffer (Aleiodes) $* \rightarrow 1465$

lancifer Haliday (Dyscoletes) $* \rightarrow 998$

lapponicus Thomson (Ichneutes) $* \rightarrow 1016$

laricis Haliday (Pauesia) $* \rightarrow 443$

larvicida Wesmael (Bracon) $* \rightarrow 594$

lateralis Haliday (Chorebus) $* \rightarrow 152$

lateralis Haliday (Glyptapanteles) $* 1166$

lateralis Reinhard (Apanteles) $\rightarrow 1056$

lathyri Griffiths (Exotela) $* \rightarrow 296$

latibilis Meyer (Perilitus) $\rightarrow 900$

latibsis Meyer (Perilitus) $\rightarrow 900$

laticarpa Telenga (Agathis) $\rightarrow 14$

laticarpus Thomson (Colastes) $* \rightarrow 959$

laticephalus Telenga (Adialytus) $\rightarrow 372$

laticeps Telenga (Bracon) $\rightarrow 606$

laticeps Thomson (Orthostigma) $* \rightarrow 315$

laticeps Wesmael (Meteorus) $\rightarrow 860$

latifemur Fischer (Dinotrema) $* \rightarrow 281$

latipes Fischer (Opius) $\rightarrow 1372$

latistigma Fischer (Eudinostigma) $* \rightarrow 290$

lativalvis Jakimavicius (Centistes) $\rightarrow 819$

latus Telenga (Aleiodes) $\rightarrow 1454$

lautellus Marshall (Pholetesor) $\rightarrow 1249$

lautus Szépligeti (Bracon) $\rightarrow 606$

lavagnei Picard (Monolexis) $\rightarrow 770$

lederi Fischer (Agathis) $\rightarrow 23$

lemantinum Gautier (Praon) $* \rightarrow 459$

lencoranus Telenga (Bracon) $\rightarrow 606$

lenea Nixon (Apanteles) $* \rightarrow 1046$

lepelleyi Waterston (Areopraon) $* \rightarrow 397$

lepidus Haliday (Eubazus) $* \rightarrow 494$

leptocephalus Hartig (Orgilus) $* \rightarrow 1426$

leptogaster Haliday (Chorebus) $* \rightarrow 153$

leptopus Thomson (Ascogaster) $\rightarrow 681$

leptostigma Ruthe (Blacus) $* \rightarrow 805$

leptostigma Wesmael (Opius) $* \rightarrow 1349$

leptus Marshall (Bracon) $* \rightarrow 595$

lestes Nixon (Dacnusa) $\rightarrow 241$

letifer Haliday (Misaphidus) $* \rightarrow 432$

leucaniae Wilkinson (Cotesia) $\rightarrow 1064$

leucogaster Nees (Doryctes) $* \rightarrow 754$

leucopterus Haliday (Diaeretus) $* \rightarrow 405$

levis Wesmael (Opius) $* \rightarrow 1350$

levis Wesmael (Pseudichneutes) $* \rightarrow 1019$ 
leviventris Wesmael (Meteorus) $\rightarrow 875$ lichasherstovi Telenga (Phaenocarpa) $* \rightarrow 335$ lictorius Reinhard (Apanteles) $\rightarrow 1044$ lignarius Ratzeburg (Spathius) $* \rightarrow 784$ lignator Lepeletier \& Serville (Helcon) $\rightarrow 999$ ligustri van Achterberg (Monoctonus) $* \rightarrow 436$ limbata Marshall (Cotesia) $* \rightarrow 1084$ limbator Ratzeburg (Macrocentrus) $\rightarrow 1021$ limitatus Wesmael (Ascogaster) $\rightarrow 697$ limnicola Nees (Chorebus) $* \rightarrow 154$ limoniadum Marshall (Chorebus) $* 155$ linearis Hellén (Aleiodes) $* \rightarrow 1466$ linearis Nees (Macrocentrus) $* \rightarrow 1031$ lineatus Reinhard (Dolichogenidea) $\rightarrow 1130$ lineipes Wesmael (Dolichogenidea) $* \rightarrow 1150$ lineola Curtis (Cotesia) $* \rightarrow 1085$ lineolata Hedwig (Cotesia) $\rightarrow 1085$ linguarius Nees (Bassus) $* \rightarrow 32$ liogaster Marshall (Doryctes) $\rightarrow 754$ lionotus Thomson (Meteorus) $* \rightarrow 865$ liopleuris Thomson (Dacnusa) $* \rightarrow 248$ liparae Giraud (Polemochartus) $* \rightarrow 348$ liparidis Bouché (Glyptapanteles) $* 1167$ liquis Ruthe (Meteorus) $\rightarrow 863$ lissogaster Tobias (Colastes) $\rightarrow 958$ lissos Nixon (Dacnusa) $* \rightarrow 249$ litae Nixon (Dolichogenidea) $\rightarrow 1125$ lithospermi Griffiths (Dacnusa) $* \rightarrow 250$ lituratus Haliday (Allurus) $* \rightarrow 789$ livida Haliday (Phaenocarpa) $* \rightarrow 336$ livida Telenga (Bracon) $* \rightarrow 596$ lividipes Förster (Opius) $\rightarrow 1371$ lividipes Wesmael (Pholetesor) $\rightarrow 1249$ lonchopterae Fischer (Alloea) $* \rightarrow 49$ lonchopterae Ruschka (Chorebus) $* \rightarrow 156$ longicauda Boheman (Vipio) $* \rightarrow 674$ longicauda Fahringer (Pholetesor) $\rightarrow 1249$ longicauda Kokujev (Agathis) $\rightarrow 10$ longicauda Ratzeburg (Apanteles) $\rightarrow 1047$ longicauda Thomson (Biosteres) $* \rightarrow 1285$ longicauda Tobias (Diospilus) $\rightarrow 987$ longicauda Wesmael (Dolichogenidea) $\rightarrow 1128$ longicauderra Shenefelt (Apanteles) $* \rightarrow 1047$ longicaudis Giraud (Ontsira) $* \rightarrow 773$ longicaudis Herrich-Schäffer (Macrocentrus) $\rightarrow 1033$ longicaudis Ratzeburg (Bracon) $\rightarrow 625$ longicaudis Ratzeburg (Eubazus) $* 495$ longicaudis Ratzeburg (Meteorus) $* \rightarrow 866$ longicollis Wesmael (Bracon) $* \rightarrow 597$ longicorne Königsmann (Orthostigma) $* \rightarrow 316$ longicorne Marshall (Praon) $\rightarrow 455$ longicornis Nees (Chorebus) $* \rightarrow 157$ longicornis Thomson (Opius) $* \rightarrow 1351$ longipalpis Reinhard (Dolichogenidea) $* \rightarrow 1151$ longipalpis Thomson (Cyanopterus) $\rightarrow 661$ longipalpis van Achterberg (Austrozele) $* \rightarrow 1020$ longipennis Gravenhorst (Blacus) $* \rightarrow 806$ longipes Fischer (Utetes) $\rightarrow 1406$ longipes Förster (Myiocephalus) $\rightarrow 881$ longiradialis Fischer (Opius) $* \rightarrow 1352$ longiradialis Nixon (Dacnusa) $* 251$ longiseta Herrich-Schäffer (Schizoprymnus) $* \rightarrow 519$ longistigma Telenga (Protodacnusa) $\rightarrow 352$ longiventris Tobias (Ascogaster) $\rightarrow 691$ longulus Marshall (Aphidius) $\rightarrow 395$ longulus Thomson (Bracon) $* \rightarrow 598$ lonicerae Fischer (Opius) $* \rightarrow 1353$ lonicerella Griffiths (Dacnusa) $* \rightarrow 252$ lophyriphagus Fahringer (Meteorus) $\rightarrow 863$ loripenne Graham (Atopandrium) $\rightarrow 91$ loripes Haliday (Alysia) $\rightarrow 57$ lucia Haliday (Alysia) $* \rightarrow 55$ luciana Nixon (Glyptapanteles) $* \rightarrow 1168$ lucicola Haliday (Alysia) $* \rightarrow 56$ lucidator Nees (Centistes) $\rightarrow 820$ lucidator Trentepohl (Meteorus) $\rightarrow 863$ lucidus Szépligeti (Apanteles) $\rightarrow 1043$ lucidus Szépligeti (Opius) $* \rightarrow 1354$ lucifugus Lyle (Glyptapanteles) $\rightarrow 1170$ luctuosa Haliday (Microgaster) $* \rightarrow 1196$ lugens Haliday (Dacnusa) $* \rightarrow 253$ lugens Haliday (Opius) $* \rightarrow 1355$ lugubrator Ratzeburg (Bassus) $\rightarrow 33$ lugubris Förster (Agathis) $* \rightarrow 15$ lugubris Nixon (Chorebus) $* \rightarrow 158$ lugubris Ruthe (Microplitis) $* \rightarrow 1227$ lugubris Wesmael (Microchelonus) $* \rightarrow 727$ luridus Ruthe (Meteorus) $\rightarrow 856$ luridus sensu Tobias (Meteorus) $\rightarrow 861$ lustrator Haliday (Colastes) $* \rightarrow 960$ luteicornis Herrich-Schäffer (Ascogaster) $\rightarrow 683$ luteipes Thomson (Triaspis) $* \rightarrow 534$ lutescens Haliday (Aphidius) $\rightarrow 376$ luteus Cameron (Charmon) $\rightarrow 679$ luteus Nees (Rogas) * $\rightarrow 1485$ luzulae Griffiths (Chorebus) $* \rightarrow 159$ lychnidis Marshall (Aphidius) $\rightarrow 385$ lyctaea Cole (Syntretus) $\rightarrow 932$ lylei Shenefelt (Protapanteles) $\rightarrow 1266$ macedonicus Fischer (Meteorus) $\rightarrow 874$ mackaueri Starý (Adialytus) $\rightarrow 371$ macrocarpus Mackauer (Diaeretellus) $* \rightarrow 402$ 
macrocephalus Nees (Eubazus) $* \rightarrow 496$ macrocera Thomson (Dinotrema) $* \rightarrow 282$ macrocerus Thomson (Eurytenes) $* \rightarrow 1302$ macrocornis Mackauer (Lysiphlebus) $\rightarrow 421$ macroptera Rudow (Orgilus) $\rightarrow 1430$ macropterus Haeselbarth (Blacus) $* \rightarrow 807$ macroscapus Ruthe (Streblocera) $* \rightarrow 919$ macrospila Haliday (Dactrusa) $* \rightarrow 254$ mactator Klug (Iphiaulax) * $\rightarrow 666$ maculator Dahlbom (Microchelonus) $\rightarrow 726$ maculatus Papp (Aleiodes) $\rightarrow 1454$ maculatus Ruthe (Microgaster) $\rightarrow 1198$ maculifer Szépligeti (Bracon) $\rightarrow 572$ maculiger Wesmael (Bracon) $\rightarrow 645$ maculipennis Szépligeti (Ontsita) $\rightarrow 774$ maculipes Haliday (Orthostigma) $* \rightarrow 317$ maculipes Thomson (Dacnusa) $* \rightarrow 255$ maculipes Wesmael (Blacus) $* \rightarrow 808$ maculipes Wesmael (Opius) $* \rightarrow 1356$ maderi Fahringer (Phanerotoma) $\rightarrow 742$ magdalenae Sterzynski (Colastes) $* \rightarrow 961$ magnicauda Fischer (Opius) $* \rightarrow 1357$ magnus Fischer (Utetes) $* \rightarrow 1408$ magnus Telenga (Dolichogenidea) $\rightarrow 1153$ majalis Wesmael (Protapanteles) $* \rightarrow 1264$ major Fonscolombe (Earinus) $\rightarrow 43$ major Szépligeti (Rhysipolis) $\rightarrow 977$ major Thomson (Aphaereta) $* \rightarrow 74$ makinoharanus Minamikawa (Meteorus) $\rightarrow 863$ malaisei Shestakov (Aleiodes) $\rightarrow 1453$ malvacearum Latreille (Agathis) $* \rightarrow 16$ mamertes Nixon (Chorebus) $\rightarrow 149$ mamestrae Matsumura (Cotesia) $\rightarrow 1109$ mamestrae Viereck (Meteorus) $\rightarrow 875$ mamillanus Ruthe (Blacus) $* \rightarrow 809$ mandanis Nixon (Protapanteles) $* \rightarrow 1265$ mandibulare Tobias (Orthostigma) $* \rightarrow 318$ mandibularis Haliday (Microchelonus) $\rightarrow 726$ mandibularis Nees (Trachionus) $* \rightarrow 367$ mandibularis Thomson (Microplitis) $* \rightarrow 1228$ mandibulator Nees (Alysia) $* \rightarrow 57$ manducator Panzer (Alysia) $* \rightarrow 58$ manevali Gautier \& Bonnamour (Microplitis) $\rightarrow 1241$ manilae Ashmead (Cotesia) $\rightarrow 1099$ marginalis Haliday (Epimicta) $* 289$ marginata Nees (Rasivalva) $* \rightarrow 1270$ marginata sensu Telenga (Diolcogaster) $\rightarrow 1121$ marginator Nees (Macrocentrus) $* \rightarrow 1032$ marginellus Wesmael (Clinocentrus) $\rightarrow 946$ marginellus Wesmael (Microgaster) $\rightarrow 1205$ maria Haliday (Phaenocarpa) $* \rightarrow 337$ mariae Dalla Torre (Bracon) $* \rightarrow 599$

maritima Haliday (Idiasta) $* \rightarrow 306$

maritimus Wilkinson (Pholetesor) $* \rightarrow 1252$

marothiensis Szépligeti (Doryctes) $\rightarrow 754$

marshalli Bignell (Protapanteles) $\rightarrow 1260$

marshalli Fahringer (Agathis) $\rightarrow 10$ marshalli Szépligeti (Bracon) $* \rightarrow 600$ maslovskii Telenga (Bracon) $\rightarrow 590$ matricariae Haliday (Aphidius) ${ }^{*} \rightarrow 385$ maxima Fischer (Dinotrema) $\rightarrow 277$ maxima Fischer (Dacnusa) $* \rightarrow 256$ maximus Muesebeck (Zele) $\rightarrow 936$ mediana Ruthe (Microplitis) $\rightarrow 1229$ medianus Ratzeburg (Apanteles) $* \rightarrow 1048$ medianus Ruthe (Meteorus) $\rightarrow 875$ medianus Thomson (Aleiodes) $\rightarrow 1471$ mediator Haliday (Microplitis) $* \rightarrow 1229$ mediator Nees (Bassus) $* \rightarrow 33$ mediator Nees (Bracon) $* \rightarrow 601$ meditator Haliday (Rhysipolis) * $\rightarrow 977$ meditertaneus Mackauer (Misaphidus) $\rightarrow 427$ megapterus Wesmael (Bracon) $* \rightarrow 602$ melanocephalus Nees (Aphidius) $\rightarrow$ incertae sedis melanocera Thomson (Antrusa) $* \rightarrow 69$ melanocerus Wesmael (Biosteres) $\rightarrow 1287$ melanophytobiae Griffiths (Chorebus) $* \rightarrow 160$ melanopus Ruthe (Perilitus) $* \rightarrow 902$ melanoscela Ratzeburg (Cotesia) $* 1086$ melanoscelus Nees (Diospilus) $* \rightarrow 990$ melanosoma Szépligeti (Biosteres) $\rightarrow 1281$ melanosoma Szépligeti (Bracon) $\rightarrow 646$ melanostictus Capron (Meteorus) $* \rightarrow 867$ melanostigma Strand (Coeloides) $* \rightarrow 654$ melanotus Wesmael (Coeloides) * $\rightarrow 655$ melas Giraud (Polemochartus) $* 349$ melbus Papp (Utetes) $\rightarrow 1406$ melicerta Nixon (Dacnusa) $* \rightarrow 257$ melitaearum Wilkinson (Cotesia) $\rightarrow 1111$ melittearum Wilkinson misspell. (Cotesia) $\rightarrow 1111$ melleus Cresson (Homolobus) $\rightarrow 1014$ mellinervis Viereck (Meteorus) $\rightarrow 875$ memnon Nixon (Cotesia) $\rightarrow$ record uncertain memorabilis Alexeev (Iconella) $\rightarrow 1180$ mendax Kokujev (Vipio) $\rightarrow 673$ meracus Fischer (Opius) $\rightarrow 1379$ merceti Quilis (Aphidius) $\rightarrow 385$ merellus Nixon (Chorebus) $* \rightarrow 161$ meridiana Haliday (Microgaster) $* \rightarrow 1197$ meridionalis Schutze \& Roman (Microgaster) $\rightarrow 1197$ meridionellae Fischer (Agathis) $\rightarrow 13$ merope Nixon (Dacnusa) $* \rightarrow 258$ 
merula Reinhard (Iconella) $* \rightarrow 1181$

mesocrinoides Goidanich (Pentapleura) $\rightarrow 326$

mesopotamicus Fischer (Meteorus) $\rightarrow 875$

mesoxanthus Ruschka (Dolichogenidea) $\rightarrow 1127$

messoria Haliday (Microgaster) $* \rightarrow 1198$

metacarpalis sensu Tobias (Apanteles) $\rightarrow 1055$

metacarpalis Thomson (Apanteles) $* \rightarrow 1049$

metallicus Jakimavicius (Pholetesor) $\rightarrow 1251$

micans Stelfox (Biosteres) $* 1286$

microcera Thomson (Aspilota) $* \rightarrow 86$

microcerus Wesmael (Meteorus) $\rightarrow 853$

microphtalmus Wesmael (Microchelonus) $* \rightarrow 728$

microphthalmus Wesmael misspell. (Microchelonus)

$\rightarrow 728$

micropterus Haliday (Meteorus) $* \rightarrow 868$

micropterus Morley (Orgilus) $\rightarrow 1430$

middendorffi Ratzeburg (Dendrosoter) $* \rightarrow 749$

mihalyii Papp (Protapanteles) $\rightarrow 1263$

miniatus Herrich-Schäffer (Aleiodes) $* \rightarrow 1467$

minimus Fischer (Opius) $\rightarrow 1396$

minimus van Achterberg \& Haeselbarth (Syntretus)

$* \rightarrow 925$

miniscula Griffiths misspell. (Exotela) $\rightarrow 297$

minor Enderlein (Bassus) $\rightarrow 32$

minor Fahringer (Chelonus) $\rightarrow 703$

minor Fahringer (Sathon) $\rightarrow 1271$

minor Fischer (Opius) $\rightarrow 1338$

minor Šnoflak (Phanerotoma) $\rightarrow 741$

minor Stelfox (Ephedrus) $\rightarrow 410$

minor Tacger (Orgilus) $* \rightarrow 1427$

minotend Shenefelt (Bassus) $\rightarrow 32$

minuscula Griffiths (Exotela) $* 297$

minuta Nees (Aphaereta) $* \rightarrow 75$

minuta Niezabitowski (Agathis) $\rightarrow 15$

minuta Reinhard (Diolcogaster) $* \rightarrow 1120$

minutator auct. (Bracon) $\rightarrow 642$

minutator Fabricius (Bracon) $* \rightarrow 603$

minutissima Tobias (Aphaereta) $\rightarrow 76$

minutissimus Ratzeburg (Ecphylus) $\rightarrow 759$

minutissimus Zetterstedt (Adelius) $\rightarrow 8$

minutor Thunberg (Meteorus) $\rightarrow 863$

minutus Costa (Microchelonus) $* \rightarrow 729$

minutus Nees (Aspilota) $\rightarrow 84$

minutus Ratzeburg (Eubazus) $* \rightarrow 497$

minutus Wesmael (Oncophanes) $* \rightarrow 1444$

mira Ruthe (Dimeris) * $\rightarrow 965$

mirabilis Fischer (Opius) $* \rightarrow 1358$

mirotarsi Starý (Aphidius) $\rightarrow 381$

misellus Marshall (Chorebus) $* \rightarrow 162$

miser Nixon (Protodacnusa) $\rightarrow 350$

mitis Haliday (Leiophron) $\rightarrow$ incertae sedis mixtus Szépligeti (Bracon) $\rightarrow 590$

mlokossewitschi Kokujev (Ascogaster) $\rightarrow 684$

mocsaryi Szépligeti (Cephaloplites) $* \rightarrow 1294$

modestus Haeselbarth (Blacus) $* \rightarrow 810$

modestus Reinhard (Aleiodes) $* \rightarrow 1468$

moestus Ratzeburg (Microplitis) $* \rightarrow 1230$

moldavicus Tobias (Colastes) $* \rightarrow 962$

moldavicus Tobias (Orgilus) $* \rightarrow 1428$

molorchi Fischer (Meteorus) $\rightarrow 878$

molorchicola Fischer (Diospilus) $* \rightarrow 991$

mongolicus Fahringer (Meteorus) $\rightarrow 852$

moniliatus Nees (Hormius) $* \rightarrow 967$

monilicornis Herrich-Schäffer (Ascogaster) $\rightarrow 686$

monilicornis Thomson (Lysiphlebus) $\rightarrow 422$

monochami Fischer (Meteorus) $\rightarrow 858$

monospila Haliday misspell. (Dacnusa) $\rightarrow 254$

monticola Szépligeti (Opius) $* 1359$

motio Reinhard (Aleiodes) $* \rightarrow 1469$

moroderi Quilis (Lysiphlebus) $\rightarrow 422$

morosus Reinhard (Diospilus) $* \rightarrow 992$

muctonatus Telenga (Chorebus) $* \rightarrow 163$

mucronatus Thomson (Eubazus) $\rightarrow 505$

muesebecki Smith (Ephedrus) $\rightarrow 409$

multiarticulata Marshall (Adelurola) $\rightarrow 46$

multiarticulatus Ratzeburg (Ascogaster) $\rightarrow 685$

multiarticulatus Ratzeburg (Iphiaulax) $\rightarrow 664$

multiarticulatus Ratzeburg (Pygostolus) * $\rightarrow 911$

mundus Förster (Opius) $* \rightarrow 1360$

muricatus Haliday (Allurus) $* 790$

murinanae Capek \& Zwölfer (Dolichogenidea)

$\rightarrow 1153$

mutabilis Nees (Chelonus) $\rightarrow 712$

mutillarius Fabricius (Spathius) $\rightarrow 783$

mutillator auct. (Doryctes) $\rightarrow 757$

mutillator Thunberg (Spathius) $\rightarrow 783$

mutuator Nees (Ademon) $\rightarrow 1273$

mutus Fischer (Opius) $\rightarrow 1312$

mygdonia Nixon (Glyptapanteles) $* \rightarrow 1169$

myles Nixon (Chorebus) $* \rightarrow 164$

myron Nixon (Apanteles) $* \rightarrow 1050$

mystacinus Wesmael (Histeromerus) $* \rightarrow 1008$

myzophagum Mackauer (Ptaon) $* \rightarrow 460$

nacheri Quilis (Ephedrus) $* \rightarrow 411$

naenia Morley (Chorebus) $\rightarrow 153$

naiadum Haliday (Chaenusa) $* \rightarrow 93$

nana Nixon (Chorebus) $* \rightarrow 165$

nanellae Tobias (Orgilus) $\rightarrow 1418$

nanivorac Fischer (Mirax) $\rightarrow 1272$

nanulus Szépligeti (Bracon) $\rightarrow 646$

nanus Reinhard (Pholetesor) $* \rightarrow 1253$

napus Quilis (Diaeretiella) $\rightarrow 404$ 
narangae Viereck (Cotesia) $\rightarrow 1099$ nasicornis Telenga (Rhamphagathis) $* \rightarrow 45$ nasutus Wesmael (Centistes) $* \rightarrow 823$ natator Schulz (Chaenusa) $* \rightarrow 94$ navicularis Nees (Chorebus) $* \rightarrow 166$ nawaii Ashmead (Cotesia) $\rightarrow 1077$ necans Mackauer (Praon) * $\rightarrow 461$ necator 'Trentepohl (Meteorus) $\rightarrow 854$ neesii Marshall (Atanycolus) $* \rightarrow 544$ neesii Reinhard (Ascogaster) $\rightarrow 693$ neesii Ruthe (Zele) $\rightarrow 937$ negativus Tobias (Aleiodes) $\rightarrow 1450$ nemorum Hartig (Glyptapanteles) $\rightarrow 1167$ neopendulus Fischer (Opius) $* \rightarrow 1361$ neopusillus Fischer (Opius) $\rightarrow 1366$ nephele Haliday (Idiasta) $* \rightarrow 307$ nereidum Haliday (Chaenusa) $* \rightarrow 95$ nerissa Nixon (Chorebus) $* \rightarrow 167$ nervosum Haliday (Dinotrema) $* \rightarrow 283$ nervosus Haliday (Monoctonus) $* \rightarrow 437$ nervulata Fischer (Aspilota) $* \rightarrow 87$ nevadensis Baker (Ephedrus) $\rightarrow 413$ nidulator Nees (Macrocentrus) $* \rightarrow 1033$ nigellus Tobias (Microchelonus) ${ }^{*} \rightarrow$ record uncertain niger Gautier, Bonnamout \& Gaumont (Ephedrus)

$$
* \rightarrow 412
$$

niger Lyle (Meteorus) $\rightarrow 867$

niger Nees (Coelinidea) $\rightarrow 219$

niger Zetterstedt (Earinus) $\rightarrow 44$

nigetrimus Fahringer (Aleiodes) $\rightarrow 1480$

nigra Lyle (Allurus) $\rightarrow 790$

nigra Nees (Agathis) $* \rightarrow 17$

nigra Nees (Coelinidea) $* \rightarrow 219$

nigra Szépligeti (Phanerotomella) $\rightarrow 744$

nigrator Szépligeti (Ascogaster) $\rightarrow 694$

nigtator Zetterstedt (Cyanopterus) $* \rightarrow 663$

nigratus Papp (Aleiodes) $\rightarrow 1463$

nigratus Wesmael (Bracon) $* \rightarrow 604$

nigrescens Hellén (Aleiodes) $\rightarrow 1482$

nigrescens Mackauer (Aphidius) $\rightarrow 395$

nigribasis Fahringer (Ascogaster) $\rightarrow 696$

nigricans Chao (Aridelus) $\rightarrow 791$

nigricans Fahringer (Aleiodes) $\rightarrow 1458$

nigricans Nees (Microgaster) $* \rightarrow 1199$

nigriceps Ashmead (Chremylus) $\rightarrow 944$

nigriceps Szépligeti (Opius) $\rightarrow 1366$

nigriceps Wesmael (Aleiodes) $\rightarrow 1456$

nigricinctus Fischer (Opius) $\rightarrow 1324$

nigricollis Thomson (Zele) $\rightarrow 938$

nigricollis Wesmael (Bracon) $* \rightarrow 605$

nigricolor Fischer (Opius) $* \rightarrow 1362$ nigricoloratus Fischer (Opius) $* \rightarrow 1363$

nigricornis Thomson (Ascogaster) $\rightarrow 694$

nigticornis Wesmael (Aleiodes) $* \rightarrow 1470$

nigricornis Wesmael (Diospilus) $* \rightarrow 993$

nigricoxis Wesmael (Eubazus) $* 498$

nigrifemur Papp (Chelonus) $\rightarrow 711$

nigrina Förster (Coloboma) $\rightarrow$ incertae sedis

nigrinus Ratzeburg (Chelonus) $* \rightarrow 710$

nigripalpis Wesmael (Aleiodes) $\rightarrow 1477$

nigripennis Dahl (Orgilus) $\rightarrow$ incertae sedis

nigripennis Wesmael (Proterops) $* 1018$

nigripes Hellén (Aleiodes) $\rightarrow 1477$

nigripes Papp (Aleiodes) $\rightarrow 1463$

nigripes Ratzeburg (Apanteles) $* \rightarrow 1051$

nigripes Ruthe (Eubazus) $\rightarrow 491$

nigritarsis Ruthe (Meteorus) $\rightarrow 872$

nigriteleus Smith (Aphidius) $\rightarrow 385$

nigrithorax Fischer (Opius) $\rightarrow 1338$

nigritorax Russo (Coeloides) $\rightarrow 652$

nigritulus Dahlbom (Microchelonus) $* \rightarrow 730$

nigriventris Nees (Cotesia) $\rightarrow 1077$

nigriventris Papp (Chelonus) $\rightarrow 703$

nigriventris Wesmael (Bracon) $* \rightarrow 606$

nigrobasalis Hellén (Aleiodes) $\rightarrow 1477$

nigromaculata Hellén (Aleiodes) $\rightarrow 1458$

nilae Telenga (Dolichogenidea) $\rightarrow 1127$

ninella Nixon (Chorebus) $* \rightarrow 168$

nipponensis Viereck (Diaeretiella) $\rightarrow 404$

nipponensis Viereck (Meteorus) $\rightarrow 874$

nitens Reinhard (Microchelonus) $\rightarrow 733$

nitetis Nixon (Dacnusa) $\rightarrow 266$

nitida Curtis (Leiophron) $* \rightarrow 838$

nitida Szépligeti (Biosteres) $\rightarrow 1292$

nitida Thomson (Phaenocarpa) * $\rightarrow 338$

nitidiusculus Szépligeti (Bracon) $\rightarrow 590$

nitidula Wesmael (Microgaster) $* \rightarrow 1200$

nitidulator Nees (Opius) $* \rightarrow 1364$

nitidulus Nees (Eatinus) $\rightarrow 43$

nitidus Gahan (Ephedrus) $\rightarrow 413$

nitidus Stelfox (Biosteres) $\rightarrow 1286$

nitidus Wesmael (Macrocentrus) $* \rightarrow 1034$

nixoni Belokobylskij \& Jervis (Agathis) $\rightarrow 17$

nobilis Griffiths (Chorebus) $* \rightarrow 169$

nobilis Haliday (Aleiodes) $* \rightarrow 1471$

nobilis Nees (Doryctes) $* \rightarrow 755$

nobilis Reinhard (Mictogaster) $* \rightarrow 1201$

noctuae Ratzeburg (Eubazus) $\rightarrow 502$

nocturnus Viereck (Leiophron) $\rightarrow 841$

nodatus Fischer (Opius) $\rightarrow 1389$

nomia Nixon (Chorebus) $* \rightarrow 170$

nominator Fabricius, 1793 not 1787 (Vipio) $\rightarrow 674$ 
nordmani Hellén (Orgilus) $\rightarrow 1436$

notabilis Förster (Alysia) $\rightarrow 62$

notha Marshall (Cotesia) $* \rightarrow 1087$

novicia Marshall (Microgaster) $* \rightarrow 1202$

novus Szépligeti (Bracon) $\rightarrow 572$

nowakowskii Griffiths (Exotela) $* \rightarrow 298$

nudator Thunberg (Zele) $\rightarrow 938$

nudiscutum Fischer (Desmiostoma) $\rightarrow 1296$

nugax Reinhard (Bassus) $* \rightarrow 34$

numen Nixon (Cotesia) $* \rightarrow 1088$

nunciator Fabricius (Helcon) $* \rightarrow 1001$

nydia Nixon (Chorebus) $* \rightarrow 171$

obfuscatus Nees (Meteotus) $* \rightarrow 869$

oblita Ruthe (Leiophron) $* \rightarrow 839$

obliteratus Nees (Doryctes) $\rightarrow 757$

obscura Griffiths (Exotela) $* \rightarrow 299$

obscurator auct. (Orgilus) $\rightarrow 1426$

obscurator Nees (Bracon) $* \rightarrow 607$

obscurator Nees (Orgilus) $* \rightarrow 1429$

obscurator Ratzeburg (Macrocentrus) $\rightarrow 1036$

obscuratus Herrich-Schäffer (Chelonus) $* \rightarrow 711$

obscurella Nees (Triaspis) $* \rightarrow 535$

obscutellus Ruthe (Meteorus) $\rightarrow 864$

obscuricornis Szépligeti (Bracon) $\rightarrow 595$

obscuriforme Quilis (Aphidius) $\rightarrow 385$

obscuxiformis Quilis (Misaphidus) $\rightarrow 427$

obscuripennis Förster (Aclitus) $* \rightarrow 370$

obscuripes Ruschka (Chorebus) $* \rightarrow 172$

obscuripes Thomson (Alysia) $\rightarrow 53$

obscuripes Thomson (Rhysipolis) $\rightarrow 977$

obscurus Nees (Apanteles) $* \rightarrow 1052$

obscurus Nees (Schizoprymnus) $* \rightarrow 520$

obscurus Szépligeti (Opius) $\rightarrow 1355$

obscurus Thomson (Schizoprymnus) $\rightarrow 518$

obsepiens Nixon (Microgaster) $\rightarrow 1210$

obsoletus Kurdjumov (Diaeretiella) $\rightarrow 404$

obsoletus sensu Ratzeburg (Adialytus) $\rightarrow 372$

obsoletus Wesmael (Misaphidus) $\rightarrow 431$

obsoletus Wesmael (Meteorus) $* \rightarrow 870$

ocellatae Bouché (Microplitis) $* \rightarrow 1231$

ocellatus Watanabe (Meteorus) $\rightarrow 877$

ocellatus Wesmael (Opius) $* \rightarrow 1365$

ochraceus Curtis (Aleiodes) $\rightarrow 1481$

ochrogaster Wesmael (Opius) $* \rightarrow 1366$

ochropes Lyle (Earinus) $\rightarrow 44$

ochropus Nees (Bracon) $* \rightarrow 608$

ochropus Thomson (Opius) $* \rightarrow 1367$

ochrostigma Wesmael (Apanteles) $\rightarrow 1060$

ochrosus Szépligeti (Bracon) $\rightarrow 615$

ocreatus Papp (Opius) $* \rightarrow 1368$

octonarius Ratzeburg (Glyptapanteles) $* \rightarrow 1170$ ocularis van Achterberg \& Haeselbarth (Syntretus)

$* \rightarrow 926$

oculator Fabricius (Chelonus) $* \rightarrow 712$

oculator sensu Panzer? (Chelonus) $\rightarrow 712$

oculatus Ruthe (Meteorus) $* \rightarrow 871$

ocyrae Nixon misspell. (Dacnusa) $\rightarrow 259$

ocyroe Nixon (Dacnusa) $* \rightarrow 259$

oehlkei Papp (Dolichogenidea) $* \rightarrow 1152$

oestmaeli Wesmael (Bracon) $\rightarrow 603$

oetztalicola Fischer (Opius) $\rightarrow 1308$

ofella Nixon (Cotesia) $* \rightarrow 1089$

oleraceus Haliday (Diospilus) $* \rightarrow 994$

olgae Kokujev (Chelonus) $* \rightarrow 713$

oligomera Fötster (Coloneura) $\rightarrow 225$

ononidis Marshall (Apanteles) $* \rightarrow 1053$

opaca Hellén (Avga) $* \rightarrow 943$

opacus Reinhard (Foersteria) $\rightarrow 510$

opacus Ruthe (Hygroplitis) $\rightarrow 1177$

opacus Thomson (Schizoprymnus) $* \rightarrow 521$

orbiculator Nees (Opius) * $\rightarrow 1369$

orbitator Nees (Clinocentrus) $\rightarrow 947$

orchesiae auct. (Leiophron) $\rightarrow 837$

orchesiae Boie (Meteorus) $\rightarrow 869$

ordinaria Ratzeburg (Cotesia) $* \rightarrow 1090$

orestes Nixon (Cotesia) $* \rightarrow 1091$

oritias Nixon (Chorebus) $* \rightarrow 173$

ornatulus Telenga (Pigeria) $\rightarrow 668$

ornatus Marshall (Leiophron) $\rightarrow$ incertae sedis

oscinellae Fischer (Blacus) $\rightarrow 804$

osculator Nees (Bracon) $* \rightarrow 609$

otiorhynchi Boudier (Pygostolus) $\rightarrow 910$

otiosus Marshall (Bracon) $\rightarrow 556$

ovalis Marshall (Chorebus) $* \rightarrow 174$

ovatus Marshall (Diospilus) $\rightarrow 988$

pachypus Schmiedeknecht (Meteorus) $\rightarrow 871$

pactus Haliday (Opius) $* \rightarrow 1370$

paganus Haliday (Blacus) $* \rightarrow 811$

palaearcticus Szépligeti (Biosteres) $\rightarrow 1284$

palaestinensis Mackauer (Ephedrus) $\rightarrow 413$

palitans Muesebeck (Praon) $\rightarrow 456$

pallicarpus Thomson (Bracon) $* \rightarrow 610$

pallicornis Nees (Leiophron) $\rightarrow 840$

pallida Ruthe (Ascogaster) $\rightarrow 680$

pallida Ruthe (Meteorus) $\rightarrow 856$

pallidator Nees (Bracon) $* \rightarrow 611$

pallidator Thunberg (Aleiodes) $* \rightarrow 1472$

pallidator Zetterstedt (Macrocentrus) $\rightarrow 1031$

pallidicornis Herrich-Schäffer (Aleiodes) $* \rightarrow 1473$

pallidicornis Marshall (Microplitis) $\rightarrow 1228$

pallidipennis Dalla Torre (Aleiodes) $\rightarrow 1473$ 
pallidipennis Herrich-Schäffer (Schizoprymnus)

$* \rightarrow 522$

pallidipes Marshall (Eubazus) $\rightarrow 499$

pallidipes Marshall (Glyptapanteles) $\rightarrow 1171$

pallidipes Marshall (Opius) $\rightarrow 1371$

pallidipes Marshall (Phaenodus) $\rightarrow 972$

pallidistigma Curtis (Leiophron) $* \rightarrow 840$

pallidus Bouché (Aleiodes) $\rightarrow 1481$

pallidus Förster (Lysitermus) $* \rightarrow 968$

pallidus Haliday (Trioxys) $* \rightarrow 477$

pallidus Hellén (Aleiodes) $\rightarrow 1454$

pallipede Rondani (Eubazus) $\rightarrow 499$

pallipes Curtis (Leiophron) $* \rightarrow 841$

pallipes Förster (Blacus) $\rightarrow 797$

pallipes Förster (Opius) $\rightarrow 1313$

pallipes Förster (Phaenodus) $* \rightarrow 972$

pallipes Haliday (Blacus) $* \rightarrow 812$

pallipes Herrich-Schäffer (Ascogaster) $\rightarrow 696$

pallipes Nees (Eubazus) $* \rightarrow 499$

pallipes Nees (Macrocentrus) $* \rightarrow 1035$

pallipes Nees (Triaspis) $* \rightarrow 536$

pallipes Reinhard (Glyptapanteles) $* \rightarrow 1171$

pallipes Wesmael (Centistes) $\rightarrow 824$

pallipes Wesmael (Meteorus) $* \rightarrow 872$

pallipes Wesmael (Opius) $* \rightarrow 1371$

pallitarsis Cresson (Zele) $\rightarrow 936$

palpator Gautier \& Bonnamour (Lipolexis) $\rightarrow 417$

palpebrator Ratzeburg (Bracon) $* \rightarrow 612$

palustris Starý (Diaeretellus) $* \rightarrow 403$

paminicus Voinovskaja-Kriger (Microchelonus) $\rightarrow 734$

pannonicus Starý (Trioxys) $* \rightarrow 478$

panzeri Jurine (Agathis) $\rightarrow 16$

panzerii Rondani (Aphidius) $\rightarrow$ incertae sedis

parallelus Lyle (Protapanteles) $* \rightarrow 1266$

paramaritima Königsmann (Idiasta) $* \rightarrow 308$

parasitellae Bouché (Apanteles) $* \rightarrow 1054$

parauctus Starý (Trioxys) $* \rightarrow 479$

parciconnis Herrich-Schäffer (Microchelonus) $* \rightarrow 731$

parcicornis Nees (Ephedrus) $\rightarrow 414$

parcicomis Ruthe (Perilitus) $* \rightarrow 903$

parvicornis Ruthe (Syntretus) $* \rightarrow 927$

parvicornis Thomson (Bracon) $* \rightarrow 613$

parvicornis Thomson (Synaldis) $* \rightarrow 357$

parvipennis Thomson (Orgilus) $* \rightarrow 1430$

parvistriga Thomson (Mictogaster) $* \rightarrow 1203$

parvulum Wesmael (Desmiostoma) $* \rightarrow 1296$

parvulus Förster (Adelius) $* \rightarrow 7$

parvulus Nees (Coelinius) $* \rightarrow 223$

parvulus Ruthe (Leiophron) $\rightarrow 840$

parvulus Ruthe (Microplitis) $\rightarrow 1236$

parvulus Ruthe (Eubazus) $* \rightarrow 500$ parvulus Shestakov (Iphiaulax) $\rightarrow 665$ paryulus Thomson (Meteorus) $\rightarrow 873$

parvulus Wesmael (Bracon) $* \rightarrow 614$

parvungula Thomson (Opius) $\rightarrow 1330$

parvungula Thomson (Chorebus) $* \rightarrow 175$

parvus Thomson (Schizoprymnus) $* \rightarrow 523$

pascuorum Marshall (Aphidius) $\rightarrow 377$

patzaki Taeger (Orgilus) $* \rightarrow 1431$

paupella Shenefelt (Centistes) $* \rightarrow 824$

pectoralis Herrich-Schäffer (Homolobus) $\rightarrow 1011$

pectoralis Nees (Charmon) $\rightarrow 679$

pectoralis Wesmael (Bracon) $* \rightarrow 615$

pedalis Cresson (Helcon) $\rightarrow 1001$

pedator Dahibom (Microchelonus) * $\rightarrow 732$

pedestris Wesmael (Spathius) $* \rightarrow 785$

pedias Nixon (Pholetesor) $\rightarrow 1248$

pedius Nixon misspell. (Pholetesor) $\rightarrow 1248$

pegomyiae Gahan (Biosteres) $\rightarrow 1291$

pelion Nixon (Chorebus) $* \rightarrow 176$

pellucens Nees (Microchelonus) $* 733$

pellucidus Ratzeburg (Bracon) $* \rightarrow 616$

pellucidus Telenga (Microplitis) $* \rightarrow 1232$

pendula Förster (Wesmaelia) $\rightarrow 935$

pendulator Latreille (Meteorus) $* \rightarrow 873$

pendulus Haliday (Opius) $* \rightarrow 1372$

pentapleuroides Fischer (Adelurola) $\rightarrow 46$

pentheri Fischer (Meteorus) $\rightarrow 879$

percontator Fischer (Opius) $\rightarrow 1349$

perdita Haliday (Anisocyrta) $* \rightarrow 67$

peregrinus Herrich-Schäffer (Perilitus) $* \rightarrow 004$

periclymenii Fischer (Opius) $\rightarrow 1389$

periscelis Reinhard (Aleiodes) $* \rightarrow$ no German record

perkinsi Nixon (Chorebus) $* \rightarrow 177$

perlustrandum Fischer (Dinotrema) * $\rightarrow 284$

peroculatus Wesmael (Bracon) $* \rightarrow 617$

perplexus Taeger (Orgilus) $* \rightarrow 1432$

persicae Froggatt (Ephedrus) $* \rightarrow 413$

persimilis Nixon (Antrusa) $\rightarrow 69$

persimilis Telenga (Bracon) $\rightarrow 606$

perspicuus Nees? (Cotesia) $\rightarrow 1089$

perspicuus Wesmael (Cotesia) $\rightarrow 1109$

petiolaris Thomson (Clinocentrus) $\rightarrow 948$

petiolaris Thomson (Atanycolus) $* \rightarrow 545$

petiolata Wollaston (Wesmaelia) $* \rightarrow 935$

petiolator Zetterstedt (Meteorus) $\rightarrow 873$

petiolatus Nees (Chorebus) $* \rightarrow 178$

petrovae Walley (Dolichogenidea) $* 1153$

petrovskii Kokujev (Doryctes) $\rightarrow 757$

phaedra Nixon (Chorebus) $* \rightarrow 179$

phaenicura Haliday misspell. (Aristelix) $\rightarrow 79$

phaetusa Nixon (Pholetesor) $* \rightarrow 1254$ 
phalangomyzi Starý (Aphidius) $* \rightarrow 386$ phaloniae Wilkinson (Dolichogenidea) $* \rightarrow 1154$ phoenicura Haliday (Aristelix) $* \rightarrow 79$ phorodontis Ashmead (Aphidius) $\rightarrow 385$ phryne Nixon (Exotela) $* \rightarrow 300$ piceaecollis Starý (Pauesia) $* \rightarrow 444$ piceator Zetterstedt (Phaenocarpa) $\rightarrow 332$ piceus Cresson (Diaeretiella) $\rightarrow 404$ piceus Fahringer (Aleiodes) $\rightarrow 1468$ piceus Marshall (Rhoptrocentrus) $* \rightarrow 779$ piceus Thomson (Opius) $\rightarrow 1371$ picinervis Haliday (Phaenocarpa) $* \rightarrow 339$ picipes auct. (Cotesia) $\rightarrow 1085$ picipes Bouché (Cotesia) $* \rightarrow 1092$ picipes Curtis (Leiophron) $* \rightarrow 842$ picipes Haliday (Macrocentrus) $\rightarrow 1025$ picipes Herrich-Schäffer (Aleiodes) $* \rightarrow 1474$ picipes Nees (Aphidius) $\rightarrow 377$ piciventris Wesmael (Hormius) $\rightarrow 967$ picta Haliday (Pauesia) $* \rightarrow 445$ picticornis Ruthe (Idiasta) $* \rightarrow 309$ picticornis Wesmael (Bracon) $* \rightarrow 618$ pictipennis Reinhard (Rhaconotus) ${ }^{*} \rightarrow 778$ pictipes Wesmael (Chelonus) $* \rightarrow 714$ pictus Herrich-Schäffer (Aleiodes) $\rightarrow 1456$ pictus Kokujev (Aleiodes) $\rightarrow 1478$ pieltaini Quilis (Lipolexis) $\rightarrow 417$ pieridis Bouché (Cotesia) $* \rightarrow 1093$ pieridis Packard (Cotesia) $\rightarrow 1077$ pieridivora Riley (Cotesia) $\rightarrow 1077$ piger Wesmael (Pigeria) $* \rightarrow 668$ pilicotnis Thomson (Cotesia) $* \rightarrow 1094$ pilosum Mackauer (Areopraon) $* \rightarrow 398$ pilosus Tobias (Earinus) $\rightarrow 43$ pimpinellae Niezabitowski (Orgilus) $* \rightarrow 1433$ pineti Thomson (Bracon) $* \rightarrow 619$ pini Haliday (Pauesia) $* \rightarrow 446$ pinicola Hedquist (Ecphylus) $\rightarrow 759$ pinicollis Starý (Pauesia) $* \rightarrow 447$ pirchitticola Fischer (Opius) $* \rightarrow 1373$ placidus Gautier (Misaphidus) $\rightarrow 427$ placidus Haliday (Cotesia) $\rightarrow 1096$ placidus Haliday (Biosteres) $* \rightarrow 1287$ plagiator Nees (Ephedrus) $* \rightarrow 414$ planiceps Marshall (Dyscritulus) $* \rightarrow 406$ planiceps Telenga (Bracon) $\rightarrow 573$ planifrons auct. (Phanerotoma) $\rightarrow 743$ planifrons Nees (Phanerotoma) * $\rightarrow 742$ planistipes Nees (Aphidius) $\rightarrow$ incertae sedis plantaginis Griffiths (Dacnusa) $* \rightarrow 260$ platypyga Šnoflak (Phanerotoma) $\rightarrow 743$ plesiorapae Blanchard (Diaeretiella) $\rightarrow 404$ pleuralis Cresson (Charmon) $\rightarrow 679$ pleuralis Ruthe (Meteorus) $\rightarrow 863$ pliginskii Telenga (Bracon) $* \rightarrow 620$ plumicornis Ruthe (Marshiella) $* \rightarrow 848$ plutellae Kurdjumov (Cotesia) $* \rightarrow 1095$ pluto Morley (Microgaster) $\rightarrow 1198$ pneumatica Fischer (Aspilota) $\rightarrow 83$ poacearum Starý (Aphidius) $\rightarrow 387$ podlussanyi Papp (Triaspis) $* \rightarrow 537$ poemyzae Griffiths (Chorebus) $* \rightarrow 180$ polita Marshall (Microgaster) $* \rightarrow 1204$ politus Ashmead (Glyptapanteles) $\rightarrow 1167$ politus Ratzeburg (Eubazus) $\rightarrow 503$ politus Ruthe (Syntretus) $* \rightarrow 928$ pollipes Kirchner (Eubazus) $\rightarrow 499$ polonicus Fahringer (Illidops) $\rightarrow 1183$ polonicus Niezabitowski (Spathius) $* \rightarrow 786$ polygoni Griffiths (Chorebus) $* \rightarrow 181$ polygoni Marshall (Aphidius) $\rightarrow 385$ polypori Gautier \& Bonnamour (Apanteles) $\rightarrow 1054$ polyzonius Wesmael (Opius) $* \rightarrow 1374$ pomarius Reinhard (Doryctes) $* \rightarrow 756$ popularis Haliday (Protapanteles) $* \rightarrow 1267$ porthetriae Muesebeck (Glyptapanteles) $* \rightarrow 1172$ pospelovi Kurdjumov (Chorebus) $* \rightarrow 182$ pospelovi Telenga (Meteorus) $\rightarrow 858$ pospelowi Telenga misspell. (Meteorus) $\rightarrow 858$ postica Nees (Microgaster) $* \rightarrow 1205$ posticae Sonan (Glyptapanteles) $\rightarrow 1167$ posticatae Fischer (Utetes) $* \rightarrow 1409$ posticus Haliday (Chorebus) $* \rightarrow 183$ praecisus Ratzeburg (Ontsira) $\rightarrow 772$ praecox Wesmael (Bracon) $\rightarrow 645$ praedo Förster (Orthostigma) $\rightarrow$ incertae sedis praepotens auct. (Cotesia) $\rightarrow 1102$ praepotens Haliday (Cotesia) $* \rightarrow 1096$ praerogator Nees (Rogas) $\rightarrow$ incertae sedis praetermissus Marshall (Bracon) $* \rightarrow 621$ praetextatus Haliday (Cotesia) $\rightarrow 1064$ praetor Reinhard (Aleiodes) $* \rightarrow 1475$ praevisus Gautier \& Bonnamour (Pauesia) $\rightarrow 450$ pratellae Curtis (Phaenocarpa) $* \rightarrow 340$ priapus Gautier \& Cleu (Sathon) $\rightarrow 1271$ prinoptus Papp (Apanteles) $* \rightarrow 1055$ procera Haliday (Coelinidea) $\rightarrow 220$ procera Ruthe (Microgaster) $* \rightarrow 1206$ procerus Costa (Macrocentrus) $\rightarrow 1033$ procerus Wesmael (Biosteres) $\rightarrow 1282$ productus Herrich-Schäffer (Chelonus) $* \rightarrow 715$ profligator Haliday (Meteorus) $\rightarrow 855$ 
propallipes Shenefelt (Blacus) $\rightarrow 797$

propinqua Kokujev (Agathis) $\rightarrow 11$

propodealis Fischer (Opius) $* \rightarrow 1375$

protaeus Wesmael (Aphidius) $\rightarrow 389$

protuberans Nees (Dendrosoter) $* \rightarrow 750$

pruni Ivanov (Praon) $\rightarrow 463$

psalliotae Telenga (Phaenocarpa) $* \rightarrow 341$

pseudolaticeps Königsmann (Othostigma) $* \rightarrow 319$

pseudomediana Fahringer (Microplitis) $\rightarrow 1229$

pseudomisellus Griffiths (Chorebus) $* \rightarrow 184$

pseudoplatani Marshall (Falciconus) $* \rightarrow 416$

pterocommae Ashmead (Aphidius) $\rightarrow 378$

pterocommae Marshall (Aphidius) $\rightarrow 378$

puber Haliday (Foersteria) $* \rightarrow 510$

pubescens Curtis (Dacnusa) $* \rightarrow 261$

pubescens Ratzeburg (Rasivalva) $\rightarrow 1269$

pubicornis Thomson (Colastes) $* \rightarrow 963$

pulchellus Curtis (Ascogaster) $\rightarrow 683$

pulchellus Griffiths (Chorebus) * $\rightarrow 185$

pulchellus Stelfox (Ephedrus) $\rightarrow 413$

pulchellus Telenga (Iphiaulax) $\rightarrow 665$

pulcherrimus Fischer (Opius) $* \rightarrow 1376$

pulchriceps Szépligeti (Opius) $* \rightarrow 1377$

pulchricornis Kolubajev (Aleiodes) $\rightarrow 1476$

pulchricornis sensu Ruthe (Meteorus) $\rightarrow 850$

pulchricornis Szépligeti (Microchelonus) $\rightarrow 733$

pulchricornis Wesmael (Meteorus) $* \rightarrow 874$

pulchripes Szepligeti (Doryctes) $\rightarrow 754$

pulchripes Wesmael (Aleiodes) $* \rightarrow 1476$

pulchrithorax Fischer (Opius) $\rightarrow 1322$

pulchriventris Fischer (Opius) $\rightarrow 1377$

pulicariae Fischer (Opius) $* \rightarrow 1378$

pullata Haliday (Phaenocarpa) $* \rightarrow 342$

pullatus Dahlbom (Schizoprymnus) $* \rightarrow 524$

pullum Förster (Dinotrema) $* \rightarrow 285$

pumila Nees (Orthostigma) $* \rightarrow 320$

pumilio Nees (Gnamptodon) $* \rightarrow 979$

pumilio Nees (Pentapleura) $* \rightarrow 326$

pumilionis Roman (Bracon) $\rightarrow 645$

pumilus Ratzeburg (Bassus) $* \rightarrow 35$

punctatus Abdinbekova (Bassus) $\rightarrow 37$

punctatus Provancher (Leiophron) $\rightarrow 841$

punctatus Ratzeburg (Eubazus) $* 501$

punctifer Thomson (Bracon) $* \rightarrow 622$

punctiger Wesmael (Dolichogenidea) $* \rightarrow 1155$

punctiscuta Thomson (Biosteres) $* \rightarrow 1288$

punctiventris Ruthe (Meteorus) $\rightarrow 851$

punctiventris Thomson (Opius) $\rightarrow 1314$

punctulator Nees (Bracon) * $\rightarrow 623$

punctulator Nees (Orgilus) $* \rightarrow 1434$

punctus Goureau (Chorebus) ${ }^{*} \rightarrow 186$ pusillimus Fahringer (Bracon) $* \rightarrow 624$

pusillum Nees (Dinotrema) $* \rightarrow 286$

pusillus Ratzeburg (Bracon) $\rightarrow 624$

pusio Marshall (Chelonus) $* \rightarrow 716$

pusio Marshall (Syntretus) $* \rightarrow 929$

pygmaeator Nees (Opius) $* \rightarrow 1379$

pygmaeus Fischer (Opius) $* \rightarrow 1380$

pygmeator Nees misspell. (Opius) $\rightarrow 1379$

pyrrhia Beirne (Adelius) $\rightarrow 4$

quadridens Herrich-Schäffer (Chelonus)

$\rightarrow$ incertae sedis

quadridentata Wesmael (Ascogaster) $* \rightarrow 694$

quinquemaculatus auct. (Bracon) $\rightarrow 577$

radialis Fischer (Opius) $* \rightarrow 1381$

radialis Jakimavicius (Orgilus) $* \rightarrow 1435$

radiorimata Telenga (Mictoplitis) $\rightarrow 1237$

radzayanus Ratzeburg (Spathius) $* \rightarrow 787$

raissa Nixon (Chorebus) $\rightarrow 136$

rapae M'Intosh (Diaeretiella) $* \rightarrow 404$

rasterata Fahringer (Apanteles) $\rightarrow 1043$

ratzeburgi Fischer (Eubazus) $\rightarrow 496$

ratzeburgi Ruthe (Microplitis) $\rightarrow 1233$

ratzeburgii Dalla Torre (Bracon) $* \rightarrow 625$

ratzeburgii Marshall (Ascogaster) $\rightarrow 696$

ratzeburgii Ruthe (Microplitis) $* \rightarrow 1233$

rea Nixon (Sarops) $* \rightarrow 353$

reclinator Ruthe (Leiophron) $* \rightarrow 843$

reclinator sensu Tobias (Leiophron) $\rightarrow 845$

reconditor Wesmael (Opius) $* 1382$

reconditus Hartig (Cotesia) $\rightarrow 1090$

reconditus Nees (Cotesia) $\rightarrow 1077$

rectinervis Telenga (Glyptapanteles) $\rightarrow 1165$

rectus Papp (Microchelonus) $\rightarrow 731$

redactor Thunberg (Helcon) $\rightarrow 999$

reductidens Fischer (Dinotrema) * $\rightarrow 287$

regius Haeselbarth (Perilitus) $* \rightarrow 905$

regularis Wesmael (Bracon) $\rightarrow 603$

reinhardi Fahringer (Rhoptrocentrus) $\rightarrow 779$

reinhardi Wilkinson (Glyptapanteles) $\rightarrow 1171$

relicta Ruthe (Leiophron) $* \rightarrow 844$

rendiles Fahringer (Phanerotoma) $\rightarrow 741$

renominatus Hincks (Aphidius) $\rightarrow 385$

reptantis Fischer (Opius) $\rightarrow$ record uncertain

resa Nixon (Chorebus) $* \rightarrow 187$

resinellae Linné (Macrocentrus) $* \rightarrow 1036$

restrictus Nees (Aphidius) $\rightarrow 390$

reticulator Nees (Aleiodes) $\rightarrow 1461$

reticulatus Muesebeck (Zele) $\rightarrow 939$

retusus Nees (Microchelonus) $* 734$

retusus Ruthe (Perilitus) $* \rightarrow 906$

reunitor Nees (Ichneutes) $* \rightarrow 1017$ 
rex Fischer (Opius) * $\rightarrow 1383$

rex Marshall (Doryctes) $\rightarrow 757$

rhagoleticolus Sachtleben (Psyttalia) $\rightarrow 1403$

thanis Nixon (Chorebus) * $\rightarrow 188$

rhodopeus Zaykov (Dolopsidea) $\rightarrow 1443$

thogaleus Marshall (Asyntactus) $* \rightarrow 89$

rhopalosiphi De Stefani (Aphidius) $* 387$

thynchiti Grese (Bracon) $\rightarrow 590$

ribis Haliday (Aphidius) $* 388$

rietscheli Mackauer (Misaphidus) $\rightarrow 426$

rimator Schiödte (Coelinius) $\rightarrow 223$

rimosus Herrich-Schäffer (Chelonus) $* \rightarrow 717$

rimulator Nees (Bracon) $* \rightarrow 626$

rimulosa Matshall (Tritaphis) $\rightarrow 1486$

rimulosa Thomson (Triaspis) $* \rightarrow 538$

ringens Haliday (Trachionus) $* \rightarrow 368$

riphaeica Tobias (Phaenocarpa) $* \rightarrow$ record uncertain

ripus Papp (Glyptapanteles) * $\rightarrow 1173$

risorius Reinhard (Microchelonus) $* \rightarrow 735$

roberti Wesmael (Bracon)* $\rightarrow 627$

robustus Haeselbarth (Blacus) $* \rightarrow 813$

robustus Ratzeburg (Eubazus) $* \rightarrow 502$

robustus Reinhard (Diospilus) $* \rightarrow 995$

romani Fahringer (Zele) $\rightarrow 936$

rondanii Giard (Chorebus) $* \rightarrow 189$

rosae Haliday (Aphidius) $* \rightarrow 389$

rosarum Nees (Aphidius) $\rightarrow 389$

rosenhaueri Ratzeburg (Pambolus) $\rightarrow 970$

rossicus Kokujev (Coeloides) $* \rightarrow 656$

rostrata sensu Nixon (Agathis) $\rightarrow 13$

rostratae Griffiths (Chorebus) $* \rightarrow 190$

rotundiventris Thomson (Utetes) $* \rightarrow 1410$

rubecula Marshall (Cotesia) $* \rightarrow 1097$

rubens Nees (Meteorus) $* \rightarrow 875$

rubens Reinhard (Glyptapanteles) $* \rightarrow 1174$

rubi Starý (Aphidius) $\rightarrow 395$

rubicola Fischer (Heterospilus) $* \rightarrow 766$

rubicundus Fischer (Heterospilus) $\rightarrow 764$

rubicundus Griffiths (Chorebus) * $\rightarrow 191$

rubidus Rossi (Spathius) $* \rightarrow 788$

rubiginosus Nees (Chremylus) $\rightarrow 944$

rubrator Ratzeburg (Orgilus) $* \rightarrow 1436$

rubriceps Ratzeburg (Meteorus) $\rightarrow 863$

rubricollis Thomson (Leiophron) $* \rightarrow 845$

rubripes Haliday (Cotesia) $* \rightarrow 1098$

rudis Tobias (Alysia) $\rightarrow 55$

rudis Wesmael (Opius) $* \rightarrow 1384$

rufa Fahringer (Bassus) $\rightarrow 39$

rufa Förster (Dirthope) $* \rightarrow 745$

rufescens Förster (Asobara) $* \rightarrow 80$

rufescens Latreille (Phanerotoma) $* \rightarrow 743$ rufescens Ruthe (Blacus) $* \rightarrow 814$

ruficeps Nees (Phaenocarpa) $* \rightarrow 344$

ruficeps Wesmael (Ascogaster) $\rightarrow 693$

ruficeps Wesmael (Rhysipolis) $\rightarrow 975$

ruficeps Wesmael (Utetes) $* \rightarrow 1411$

ruficollis Hellén (Aleiodes) $\rightarrow 1477$

ruficollis Herrich-Schäffer (Coelinidea) $* \rightarrow 220$

ruficoloratus Fischer (Meteorus) $\rightarrow 865$

ruficornis Herrich-Schäffer (Aleiodes) $* \rightarrow 1477$

ruficornis Kirchner (Eubazus) $\rightarrow 503$

ruficornis Nees (Apanteles) $* \rightarrow 1056$

ruficornis Nees (Aspilota) $* \rightarrow 88$

ruficornis Nees (Blacus) $* \rightarrow 815$

ruficornis Szépligeti (Diospilus) $\rightarrow 994$

ruficoxis Fahringer (Bassus) $\rightarrow 39$

ruficoxis Fahringer (Earinus) $\rightarrow 44$

ruficoxis Ruthe (Microgaster) ${ }^{*} \rightarrow 1207$

ruficoxis Wesmael (Eubazus) $* \rightarrow 503$

ruficrus Haliday (Cotesia) $* \rightarrow 1099$

rufidens Nees (Alysia) $* \rightarrow 59$

rufidens Wesmael (Ascogaster) $* \rightarrow 695$

rufigaster Dahl (Phaenodus) $\rightarrow 972$

rufigaster Szépligeti (Bracon) $\rightarrow 603$

rufilabris Haliday (Mirax) $* \rightarrow 1272$

rufilabris Ratzeburg (Apanteles) $\rightarrow 1054$

rufina Fahringer (Orgilus) $\rightarrow 1441$

rufinotata Haliday (Tanycarpa) $* \rightarrow 364$

rufinotatus Curtis (Coelinidea) $\rightarrow 216$

rufipalpis Nees (Agathis) $* \rightarrow 18$

rufipalpis Szépligeti (Bracon) $\rightarrow 595$

rufipedator Szépligeti (Bracon) $\rightarrow 595$

rufipes auct. (Ascogaster) $\rightarrow 694$

rufipes Herrich-Schäffer (Ascogaster) $\rightarrow 695$

rufipes Herrich-Schäffer (Eubazus) $\rightarrow 505$

rufipes Herrich-Schäffer (Perilitus) $\rightarrow 891$

rufipes Herrich-Schäffer (Schizoprymnus) $\rightarrow 524$

rufipes Ivanov (Agathis) $\rightarrow 23$

rufipes Latreille (Ascogaster) $* \rightarrow 696$

rufipes Nees (Microgaster) $\rightarrow 1194$

rufipes Nees (Bassus) $* \rightarrow 36$

rufipes Nees (Chorebus) $* \rightarrow 192$

rufipes Reinhard (Diospilus) $\rightarrow 993$

rufipes sensu Reinhard (Ascogaster) $\rightarrow 685$

rufipes Wesmael (Opius) $* \rightarrow 1385$

rufipes Zetterstedt (Meteorus) $\rightarrow 853$

rufiscapus Szépligeti (Bracon) $\rightarrow 590$

rufithorax Telenga (Centistes) $* \rightarrow 825$

rufiventris Abdinbekova (Bassus) $\rightarrow 34$

rufiventris Fahringer (Aleiodes) $\rightarrow 1454$

rufiventris Herrich-Schäffer (Ascogaster) $\rightarrow 680$

rufiventris Nees (Dapsilarthra) $* \rightarrow 272$ 
rufiventris Telenga (Ascogaster) $\rightarrow 684$ rufocephalus Telenga (Taphaeus) $* \rightarrow 1006$ rufofasciata Hellén (Aleiodes) $\rightarrow 1458$ rufulus Thomson (Zele) $\rightarrow 936$ rufus De Geer (Meteorus) $* \rightarrow 876$ rufus Papp (Aleiodes) $\rightarrow 1480$ rugator Ratzeburg (Helconidea) $\rightarrow 1003$ rugator Ratzeburg (Macrocentrus) $\rightarrow 1032$ rugator Ratzeburg (Meteorus) $\rightarrow 880$ rugiferum Wesmael (Phanerotoma) $\rightarrow 743$ rugigena Thomson (Chelonus) $\rightarrow 718$ rugiventris Thomson (Coleopius) $* \rightarrow 1295$ rugosulus Goureau (Ascogaster) $\rightarrow 696$ rugosus Förster (Polystenus) $* \rightarrow 775$ rugosus Nees (Orgilus) $* \rightarrow 1437$ rugosus Ratzeburg (Eubazus) $* \rightarrow 504$ rugosus Thomson (Diospilus) * $\rightarrow 996$ rugulosa Fahringer (Orgilus) $\rightarrow 1426$ rugulosus Hellén (Phaenodus) * $\rightarrow 973$ rugulosus Nees (Aleiodes) $* \rightarrow 1478$ rugulosus Nees (Bassus) * $\rightarrow 37$ rugulosus Nees (Hygroplitis) $* \rightarrow 1177$ rugulosus Szépligeti (Bracon) $\rightarrow 597$ ruricola Lyle (Microplitis) $\rightarrow 1221$ ruspator Linné (Helconidea) $* \rightarrow 1004$ russatus Haliday (Hygroplitis) $* \rightarrow 1178$ rusticus Haliday (Biosteres) ${ }^{*} \rightarrow 1289$ rutheana Fahringer (Microplitis) $\rightarrow 1227$ ruthei Griffiths (Protodacnusa) $* \rightarrow 351$ ruthei Loan (Leiophron) $\rightarrow 835$ ruthei Schmiedeknecht (Meteorus) $\rightarrow 851$ rutilus Nees (Perilitus) $* \rightarrow 907$ saevulus Fischer (Opius) $* \rightarrow 1386$ saevus Haliday (Opius) $* \rightarrow 1387$ salebrosa Marshall (Cotesia) $* \rightarrow 1100$ salicaphis Fitch (Adialytus) $* \rightarrow 372$ salicis Haliday (Aphidius) $* \rightarrow 390$ salicorniae Schmiedeknecht (Meteorus) $* \rightarrow 877$ saltator Fabricius (Cardiochiles) $* \rightarrow 676$ saltator Thunberg (Cotesia) $* \rightarrow 1101$ salverdensis Hedqqist (Dolichogenidea) $\rightarrow 1125$ saponariellae Taeger (Orgilus) $* \rightarrow 1438$ sarcitorius Telenga (Apanteles) $\rightarrow 1042$ sasakawai Takada (Dacnusa) * $\rightarrow 262$ satanas Wesmael (Bracon) $\rightarrow 575$ saxo Reinhard (Centistes) $\rightarrow 823$ scaber Muesebeck (Orgilus) $\rightarrow 1420$ scaber Nees (Chelonus) $\rightarrow 718$ scaber Thomson (Bracon) $\rightarrow 628$ scaberrimus Tobias (Microchelonus)

$* \rightarrow$ record uncertain scabiosae Griffiths (Chorebus) $* \rightarrow 193$ scabrator Fabricius (Chelonus) $* \rightarrow 718$ scabricula Dahlbom (Ascogaster) $* \rightarrow 697$ scabriculus Wesmael (Biosteres) $* \rightarrow 1290$ scabriusculus Dalla Torre (Bracon) $* \rightarrow 628$ scabriusculus Zetterstedt (Ascogaster) $\rightarrow 685$ scaptomyzae Fischer (Aphaereta) $* \rightarrow 76$ scapularis Bouché (Dolichogenidea) $\rightarrow 1133$ schimitscheki Starý (Aphidius) * $\rightarrow 391$ schmiedeknechti Fahringer (Bracon) $* \rightarrow 629$ schmiedeknechti Kokujev (Agathis) $\rightarrow 13$ schmiedeknechti Szépligeti (Chelonus) $\rightarrow 713$ scolyticida Wesmael (Coeloides) $* \rightarrow 657$ scotica Marshall (Diolcogaster) $* \rightarrow 1121$ scrutator Tobias (Utetes) $\rightarrow 1408$ sculptiventris Tobias (Colastes) $\rightarrow 956$ sculpturatum Tobias (Orthostigma) $* \rightarrow 321$ sculpturatus Thomson (Atanycolus) $* \rightarrow 546$ scutatus Costa (Meteorus) $\rightarrow 875$ scutellaris Ratzeburg (Bracon) $\rightarrow 593$ scutellator Nees (Meteorus) $\rightarrow 873$ scymni Ferrière (Centistes) $* \rightarrow 826$ secalis Linné (Cenocoelius) $* \rightarrow 678$ secalis sensu Haliday (Perilitus) $\rightarrow 891$ secutor Marshall (Microchelonus) $\rightarrow 732$ seebensteinensis Fischer (Utetes) $\rightarrow 1409$ segregatus Telenga (Bracon) $\rightarrow 590$ semeyticus Jakimavicius (Colastes) $\rightarrow 955$ semiaciculata Ivanov (Agathis) * $\rightarrow 19$ semiaciculatus Stelfox (Opius) $\rightarrow 1328$ semicircularis Ratzeburg (Microplitis) $* \rightarrow 1234$ semiflavus Thomson (Bracon) $\rightarrow 599$ semirugosa Haliday (Synelix) $* \rightarrow 360$ semirugosus Nees (Eubazus) $* \rightarrow 505$ semistriatus Haliday (Eubazus) $\rightarrow 499$ senecionis Griffiths (Exotela) $* 301$ senilis Nees (Chorebus) $* \rightarrow 194$ separandus Fischer (Zele) $\rightarrow 939$ sera Nixon (Chorebus) $* \rightarrow 195$ seriatus Herrich-Schäffer (Aleiodes) $* \rightarrow 1479$ sericea Nees (Cotesia) $* \rightarrow 1102$ sericeus Marshall (Cotesia) $\rightarrow 1094$ seriphia Nixon (Apanteles) $* \rightarrow$ no German tecord serratulae Tobias (Agathis) $\rightarrow 23$ sesostris Nixon (1llidops) $\rightarrow 1183$ sessilis Geoffroy? (Cotesia) $\rightarrow 1082$ setiger Mackauer (Aphidius) $* \rightarrow 392$ seuratii Marshall (Microplitis) $\rightarrow 1236$ sibirica Telenga (Dacnusa) $* \rightarrow 263$ sibiricus Fahringer (Dolichogenidea) $\rightarrow 1130$ sibiricus Fahringer (Zele) $\rightarrow 937$ 
sibiricus Kokujev (Hypodoryctes) $* \rightarrow 768$ sibiricus Kokujev (Aleiodes) ${ }^{*} \rightarrow$ record uncertain sibiricus Papp (Glyptapanteles) $* \rightarrow 1175$ sibiricus Telenga (Cardiochiles) $\rightarrow 676$ sibyllarum Wilkinson (Cotesia) $* \rightarrow 1103$ sicaria Marshall (Dolichogenidea) $* \rightarrow 1156$ sicarius Mackauer (Aphidius) $\rightarrow 375$ sicheli Giard (Perilitus) $\rightarrow 898$ sigalphoides Marshall (Asyntactus) $* \rightarrow 90$ sigalphoides Marshall (Eubazus) ${ }^{*} \rightarrow 506$ sigmodus Papp (Opius) $* \rightarrow 1388$ signatus Nees (Aleiodes) $* \rightarrow 1480$ signatus Szépligeti (Atanycolus) $\rightarrow 546$ sileseacus Ratzeburg misspell. (Ecphylus) $\rightarrow 759$ silesiacus Ratzeburg (Ecphylus) $* \rightarrow 759$ silvaticus Starý (Aphidius) $\rightarrow 395$ silvestris Starý (Pauesia) $* \rightarrow 448$ silvicola Szépligeti (Opius) $\rightarrow 1323$ similator Nees (Meteorus) $\rightarrow 853$ similiformis Fischer (Opius) $\rightarrow 1389$ similis Curtis (Alysia) $\rightarrow 58$ similis Curtis (Coelinidea) $\rightarrow 216$ similis Curtis (Aleiodes) $* \rightarrow 1481$ similis Curtis (Leiophron) $* \rightarrow 846$ similis Herrich-Schäffer (Ascogaster) $\rightarrow 681$ similis Nees (Alysia) $* 60$ similis Nees (Ascogaster) $\rightarrow$ incertae sedis similis Ratzeburg (Chelonus) $\rightarrow$ incertae sedis similis Szépligeti (Aleiodes) $\rightarrow 1461$ similis Szépligeti (Dyscoletes) $\rightarrow 998$ similis Szépligeti (Rhysipolis) $\rightarrow 977$ similis Szépligeti (Triaspis) $\rightarrow 535$ similis Szépligeti (Opius) $* 1389$ similis Szépligeti (Hormius) ${ }^{*} \rightarrow$ record uncertain simillima Enderlein (Homolobus) $\rightarrow 1014$ simillimus Taeger (Orgilus) $* \rightarrow 1439$ similoides Fischer (Opius) $* \rightarrow 1390$ simplex Herrich-Schäffer (Homolobus) $\rightarrow 1009$ simulatrix Kokujev (Agathis) $\rightarrow 23$ singularis Wesmael (Opius) $* \rightarrow 1391$ sinuatus Förster (Polydegmon) $* \rightarrow 512$ sinuatus Nees (Bracon) $* \rightarrow 630$ sispes Nixon (Microplitis) $\rightarrow 1226$ snoflaki Shenefelt (Phanerotoma) $\rightarrow 742$ sodalis Haliday (Apanteles) $* \rightarrow 1057$ soenderupianus Fischer (Opius) $* \rightarrow 1392$ sofron Nixon (Microplitis) $* \rightarrow 1235$ soldanellae Griffiths (Dacnusa) $* \rightarrow 264$ solitarius Ratzeburg (Cotesia) $\rightarrow 1086$ solstitialis Stelfox (Chorebus) $* \rightarrow 196$ sonchi Marshall (Aphidius) $* \rightarrow 393$ sonchina Griffiths (Exotela) $* \rightarrow 302$

sophia Haliday (Alysia) $* \rightarrow 61$ sordidator Ratzeburg auct. (Coeloides) $\rightarrow 654$ sordipes Niees (Microplitis) $\rightarrow 1217$ sordipes Thomson (Orthostigma) $* \rightarrow 322$ soror Telenga (Ascogaster) $\rightarrow 696$ spartii Haliday (Mirax) $\rightarrow 1272$ spathiiformis Ratzeburg (Ontsira) $* \rightarrow 774$ spathuliformis Haliday (Aleiodes) $\rightarrow 1481$ spatulata Sedlag \& Stary (Pauesia) $* \rightarrow 449$ spectabilis Haliday (Microplitis) $* \rightarrow 1236$ specularis Szépligeti (Cotesia) $* \rightarrow 1104$ speculator Haliday (Taphaeus) $\rightarrow 1005$ speculator Marshall (Chelonus) $\rightarrow 707$ speculum Haliday (Dinotrema) $* \rightarrow 288$ speerschneideri Schmiedeknecht (Bracon) $* \rightarrow 631$ sphaerocephalus Szépligeti (Bracon) $\rightarrow 606$ spinaciae Thomson (Biosteres) $* \rightarrow 1291$ spinator Lepeletier \& Serville (Wroughtonia) $* \rightarrow 1007$ spinifer Nixon (Exotela) $* \rightarrow 303$ spinifer Thomson (Blacus) $\rightarrow 814$ spinifer Tobias (Ascogaster) $\rightarrow 690$ spiniphorae Fischer (Dinottema) $\rightarrow 275$ spinolae Haliday (Microgaster) $\rightarrow 1197$ spinolae Nees (Microplitis) $* \rightarrow 1237$ spinolae Ratzeburg (Microplitis) $\rightarrow 1233$ spinosum Mackauer (Praon) $* \rightarrow 462$ splendens Costa (Zele) $\rightarrow 938$ spretus Haliday (Opius) $\rightarrow 1391$ spuria Wesmael (Cotesia) $* \rightarrow 1105$ stabilis Wesmael (Bracon) $* \rightarrow 632$ stagnalis Heymons (Chorebus) $* \rightarrow 197$ staryi Fischer (Opius) $* \rightarrow 1393$ stauropodis Bridgman (Glyptapanteles) $\rightarrow 1170$ stelfoxi Haeselbarth (Blacus) $* \rightarrow 816$ stellatarum Bouché (Cotesia) $\rightarrow 1077$ stelleri Loan (Perilitus) $* \rightarrow 908$ stenocentrus Thomson (Chorebus) $* \rightarrow 198$ stenochora van Achterberg \& Haeselbarth

(Syntretus) $* \rightarrow 930$

stenostigma Thomson (Coelinidea) $* \rightarrow 221$ stercoraria Latreille (Alysia) $\rightarrow 58$ sternalis Thomson (Ascogaster) $\rightarrow 698$ stictica Ruthe (Microgaster) $* \rightarrow 1208$ sticticator Thunberg (Pygostolus) $\rightarrow 912$ sticticus Fabricius (Pygostolus) $* \rightarrow 912$ sticticus Taeger (Orgilus) $* \rightarrow 1440$ stigmaticus auct. (Microplitis) $\rightarrow 1235$ stigmaticus Hellén (Coeloides) $\rightarrow 654$ stigmaticus Ratzeburg? (Microplitis) $\rightarrow 1242$ stigmativetus Shenefelt? (Microplitis) $\rightarrow 1242$ 
stilata Förster misspell. (Coloneura) $\rightarrow 225$ stilifer Griffiths (Chorebus) $* \rightarrow 199$ stramineipes Haliday (Dacnusa) $* \rightarrow 265$ stramineipes Thomson (Opius) $\rightarrow 1320$ strandi Fahringer (Spathius) $\rightarrow 783$ strenuus Reinhard (Microplitis) ${ }^{*} \rightarrow 1238$ striatelloides Strand (Doryctes) $\rightarrow 757$ striatellus Nees (Doryctes) $* \rightarrow 757$ striatiscuta Fahringer (Microchelonus) $* \rightarrow 736$ striatoscutellaris Zilahi-Kiss (Microgaster) $\rightarrow 1185$ striatula Haliday (Laotris) $* \rightarrow 311$ striatula Nees (Triaspis) $* \rightarrow 539$ striatum Förster (Diachasma) $* \rightarrow 1299$ striatus Thomson (Meteorus) $\rightarrow 874$ strictus Stelfox (Blacus) ${ }^{*} \rightarrow 817$ strigator Thomson (Eubazus) $\rightarrow 502$ strigatus Kokujev (Doryctes) $\rightarrow 757$ strigosa Fahringer (Schizoprymnus) $\rightarrow 522$ striola Thomson (Triaspis) $* \rightarrow 540$ striolata Shestakov (Agathis) $\rightarrow 19$ striolatus Thomson (Bracon) $\rightarrow 575$ striolatus Thomson (Clinocentrus) $\rightarrow 947$ strobilorum Ratzeburg (Coeloides) $* \rightarrow 658$ stshegolevi Telenga (Ceratobracon) $* \rightarrow 649$ stygicus Loan (Leiophron) $\rightarrow 844$ stylata Förster (Coloneura) $* \rightarrow 225$ subaffinis Fischer (Opius) ${ }^{*} \rightarrow$ record uncertain subcompleta Nees (Microgaster) $* \rightarrow 1209$ subcylindricus Wesmael (Bracon) $\rightarrow 597$ subemarginatus Herrich-Schäffer (Microchelonus) $\rightarrow 734$

subfasciatus Haliday (Adelius) $* \rightarrow 8$ subfuscus Griffiths (Chorebus) $* \rightarrow 200$ subglaber Szépligeti (Bracon) $* \rightarrow 633$ subincompletus Ratzeburg (Microgaster) $\rightarrow 1194$ submuticus auct. (Chelonus) $\rightarrow 715$ subrugosus Szépligeti (Bracon) $* \rightarrow 634$ subsulcata Förster (Opius) $\rightarrow 1371$ subsulcatus Herrich-Schäffer (Microchelonus) $* \rightarrow 737$ subtilipunctata Papp (Microgaster) $* \rightarrow 1210$ subtilinugosus Papp (Orgilus) $\rightarrow 1425$ subtilis Förster (Dapsilarthra) $* \rightarrow 273$ subtilis Szépligeti (Opius) $\rightarrow 1314$ subucola Curtis (Aleiodes) $\rightarrow 1481$ suevus Reinhard (llidops) $* \rightarrow 1183$ suffolciensis Morley (Apanteles) $\rightarrow 1044$ sulcatus Curtis (Hecabolus) $* \rightarrow 760$ sulcatus Jurine (Microchelonus) $* \rightarrow 738$ sulcatus Szépligeti (Meteorus) $* \rightarrow 878$ sulcifera Papp (Aulonotus) $\rightarrow 1274$ superciliosus Wesmael (Bracon) $\rightarrow 573$ suspectus Szépligeti (Bracon) $\rightarrow 590$ suturalis Szépligeti (Chelonus) $\rightarrow 706$ swammerdamiae Muesebeck (Microgaster) $\rightarrow 1202$ sydneyensis Cameron (Cotesia) $\rightarrow 1099$ sylvaticus Haliday (Biosteres) $* \rightarrow 1292$ sylvestris Griffiths (Chorebus) $* \rightarrow 201$ sylvia Haliday (Dapsilarthra) $* \rightarrow 274$ synchitae Hedquist (Eubazus) $\rightarrow 496$ syngenesiae Nees (Agathis) $* \rightarrow 20$ syngenesii Nees misspell. (Agathis) $\rightarrow 20$ syriaca Fischer (Agathis) $\rightarrow 10$ syrmiensis Szépligeti (Rhoptrocentrus) $\rightarrow 779$ szaboi Papp (Apanteles)* $\rightarrow$ record uncertain szechuanensis Fahringer (Meteorus) $\rightarrow 875$ szoecsi Papp (Pholetesor) $\rightarrow 1253$ tabida Nees (Asobara) $* \rightarrow 81$ tabidus Haliday (Dotyctes) $\rightarrow 757$ tabidus Wesmael (Meteorus) $* \rightarrow 879$ tadzhica Telenga (Agathis) $\rightarrow 20$ tadzhicus Telenga (Dolichogenidea) $\rightarrow 1151$ taegeri van Achterberg \& Haeselbarth

(Syntretus) $* \rightarrow 931$ taeniata Förster (Opius) $\rightarrow 1385$ taiwanensis Chou \& Sharkey (Agathis) $\rightarrow 10$ talaris Haliday (Chorebus) $* \rightarrow 202$ talitzkii Telenga (Bracon) $\rightarrow 573$ talitzkii Tobias (Foersteria) $\rightarrow 510$ talitzkii Tobias (Lysitermus) $\rightarrow 968$ tanacetarius Mackauer (Aphidius) $* \rightarrow 394$ tanaceti Curtis nom. nud. (Aphidius) $\rightarrow 394$ tanaceticola Starý (Aphidius) $\rightarrow 394$ tanis Nixon (Chorebus) $* \rightarrow 203$ tardator Nees (Helcon) $* \rightarrow 1002$ tarsalis Thomson (Dacnusa) $* \rightarrow 266$ tarsator Thomson (Biosteres) $\rightarrow 1287$ tarsator Thomson (Bracon) $\rightarrow 639$ tates Nixon (Coloneura) $\rightarrow 225$ tatrica Niezabitowski (Phaenocarpa) $\rightarrow 331$ tau Ratzeburg (Microplitis) $\rightarrow 1217$ tauricus Telenga (Bracon) $\rightarrow 633$ tauricus Telenga (Heterospilus) $* \rightarrow 767$ tedellae Nixon (Apanteles) $* \rightarrow 1058$ tegularis Thomson (Bassus) $* \rightarrow 38$ telengai Muljarskaya (Bracon) $* \rightarrow 635$ telengai Tobias (Cotesia) $* \rightarrow 1106$ temporalis Telenga (Bracon) $\rightarrow 609$ temporalis Tobias (Orgilus) $* \rightarrow 1441$ temula Haliday (Dacnusa) $* \rightarrow 267$ tenebrosa Wesmael (Cotesia) $* \rightarrow 1107$ tenellae Fischer (Opius) * $\rightarrow 1395$ tenellus Marshall (Meteorus) $\rightarrow 854$ 
tener Kokujev (Aleiodes) $\rightarrow 1454$ tener Szépligeti (Bracon) $* \rightarrow 636$ tentator Rossi (Vipio)* $\rightarrow$ tecord uncertain tenthredinum Hartig (Eubazus) $\rightarrow 502$ tenucornis Förster (Pentapleura) $* \rightarrow 327$ tenuicornis Nixon (Aphaereta) $* \rightarrow 77$ tenuicornis Thomson (Meteorus) $\rightarrow 864$ tenuipes Thomson (Microplitis) $\rightarrow 1244$ tenuis Förster (Adialytus) $* \rightarrow 373$ tenuis Ratzeburg (Macrocentrus) $\rightarrow 1031$ terebella Wesmael (Bracon) $* \rightarrow 637$ terebrator Ratzeburg (Dolichogenidea) $\rightarrow 1128$ terebrator Ruthe (Blacus) $\rightarrow 800$ terminalis Ashmead (Chremylus) $\rightarrow 944$ terminatus Nees (Dinocampus) $\rightarrow 828$ terrefactor Villers (Vipio) ${ }^{*} \rightarrow 675$ tersus Förster (Opius) * $\rightarrow 1396$ tersus Reinhard (Ascogaster) $\rightarrow$ incertae sedis tesmia Nixon (Chorebus) $\rightarrow 123$ tessula Fallén (Dacnusa) $\rightarrow$ nomen falsum testacea Fahringer (Dolopsidea) $\rightarrow 1443$ testacea Griffiths (Dapsilarthra) $\rightarrow 273$ testaceator auct. (Homolobus) $\rightarrow 1009$ testaceator Curtis (Zele) $\rightarrow 936$ testaceipes Cameron (Microplitis) $\rightarrow 1236$ testaceipes Fischer (Agathis) $\rightarrow 17$ testaceus Fabricius (Rogas) $\rightarrow 1485$ testaceus Nees (Phaenocarpa) $\rightarrow 344$ testaceus sensu Spinola (Aleiodes) $\rightarrow 1456$ testaceus Stelfox (Trioxys) $\rightarrow 470$ testaceus Telenga (Heterospilus) $\rightarrow 764$ testaceus Wesmael (Utetes) $* \rightarrow 1412$ testatrix Schulz (Zele) $\rightarrow 936$ tetrica Reinhard (Cotesia) $* \rightarrow 1108$ tetropis Fischer (Caenophanes) $\rightarrow 746$ thecla Nixon (Chorebus) $* \rightarrow 204$ thelaxis Starý (Adialytus) $* \rightarrow 374$ theodori Snellen van Vollenhoven (Phaenocatpa) * $\rightarrow 345$

thienemanni Ruschka (Chorebus) $\rightarrow 209$ thisbe Nixon (Chorebus) $* \rightarrow 205$ thomsoni Marshall (Bracon) $\rightarrow 614$ thomsoni Marshall (Meteorus) $\rightarrow 874$ thomsoni Szépligeti (Colastes) $\rightarrow 960$ thomsonii Dalla Torre (Bracon) $\rightarrow 577$ thoracica Lucas (Agathis) $\rightarrow 22$ thoracicus Curtis (Meteorus) $\rightarrow 869$ thoracicus Nees (Earinus) $\rightarrow 43$ thoracicus Nees (Macrocentrus) $* 1037$ thuringiacus Schmiedeknecht (Meteorus) $\rightarrow 852$ thutingiacus Schmiedeknecht (Bracon) $* \rightarrow 638$ thusa Nixon (Chorebus) $* \rightarrow 206$

tibialis Curtis (Cotesia) $* \rightarrow 1109$ tibialis Haliday (Eubazus) $* \rightarrow 507$ tibialis Nees (Microgaster) $\rightarrow 1198$ tibialis Nees (Agathis) $* \rightarrow 21$ tibialis Zetterstedt (Bracon) $\rightarrow 575$ tineavora Nagamori (Chremylus) $\rightarrow 944$ tipulae Scopoli (Alysia) * $\rightarrow 62$ tipulator Zetterstedt (Blacus) $\rightarrow 815$ tiro Reinhard (Apanteles) $* \rightarrow 1059$ tirolensis Fischer (Opius) $* \rightarrow 1397$ titubans Wesmael (Bracon) $* \rightarrow 639$ tobiasi Belokobylskij (Heterospilus) $\rightarrow 766$ tobiasi Taeger (Orgilus) $* \rightarrow 1442$ torkai Papp (Cyanopterus) $* \rightarrow$ no German record tornator Marshall (Bracon) $* \rightarrow 640$ townesi van Achterberg \& Hacselbarth

(Macrocentrus) $* \rightarrow 1038$ transcaspicus Telenga (Aphidius) $\rightarrow 379$ transcaspicus Telenga (Bracon) $\rightarrow 573$ transversus Nixon (Chorebus) $* \rightarrow 207$ transversus Say (Chremylus) $\rightarrow 944$ triangularis Nees (Bracon) $* \rightarrow 641$ triangulator Nees (Alysia) $* \rightarrow 63$ triangulator Wesmael (Protapanteles) $* \rightarrow 1268$ tricolor Ruthe (Pambolus) $* \rightarrow 971$ tricolor Tobias (Microchelonus) $\rightarrow 726$ tricolor Wesmael (Triraphis) $* \rightarrow 1486$ trigonus Nees (Microtypus) $* \rightarrow 1416$ trilobomyzae Griffiths (Chorebus) * $\rightarrow 208$ trioxiformis Starý \& Sedlag (Metaphidius) $\rightarrow 425$ tripudians Haliday (Blacus) $* \rightarrow 818$ tristis Nees (Microplitis) * $\rightarrow 1239$ tristis Nees (Protodacnusa) $* \rightarrow 352$ tristis Wesmael (Aleiodes) $\rightarrow 1457$ trisulcus Thomson (Utetes) ${ }^{*} \rightarrow 1413$ trivialis Haliday (Blacus) $\rightarrow 802$ trivittata Ruthe (Meteorus) $\rightarrow 856$ trochanterata Thomson (Microplitis) $\rightarrow 1241$ trucidator Marshall (Bracon) $* \rightarrow 642$ truncator Nees (Alysia) $* \rightarrow 64$ truncator Ruthe (Leiophron) $* \rightarrow 847$ truncator Say (Homolobus) $* \rightarrow 1014$ truncatus Thomson (Eubazus) $\rightarrow 505$ truncatus Wesmael (Utetes) $* \rightarrow 1414$ trypetanus Fahringer (Bracon) $* \rightarrow 643$ tuberculator Zetterstedt (Eubazus) $\rightarrow 505$ tuberculatus Abdinbekova (Diospilus) $* \rightarrow 997$ tuberculatus Bouché (Microplitis) * $\rightarrow 1240$ tuberculatus Wesmael (Blacus) $\rightarrow 812$ tuberculatus Wesmael (Earinus) $\rightarrow 44$ 
tuberculatus Wesmael (Rhyssalus) $\rightarrow 1446$ tuberculifer Fischer (Meteorus) $\rightarrow 874$ tuberculifer Fischer (Opius) $* \rightarrow 1398$ tuberculifer Marshall (Leiophron) $\rightarrow 841$ tuberculifer Wesmael (Microplitis) $* \rightarrow 1241$ tuberculifera Wesmael misspell. (Microplitis) $\rightarrow 1241$ tumidulus Nees (Bassus) ${ }^{*} \rightarrow 39$ turanicus Telenga (Macrocentrus) $\rightarrow 1027$ turcicus Fischer (Opius) $* \rightarrow 1399$ turcmenicus Fischer (Opius) $\rightarrow 1356$ turissa Nixon (Chorebus) $\rightarrow 101$ turkestanicus Telenga (Doryctes) $\rightarrow 754$ ukrainicus Tobias (Orgilus) $\rightarrow 1422$ uliginosus Haliday (Chorebus) $* \rightarrow 209$ ulmi Marshall (Aphidius) $\rightarrow 381$ ultericus Telenga (Apanteles) $\rightarrow 1042$ ultor Förster (Biosteres) $\rightarrow 1293$ ultor Reinhard (Dolichogenidea) $* \rightarrow 1157$ uma Nixon (Chorebus) * $\rightarrow 210$ umbellatarum Haliday (Pholetesor) $\rightarrow 1248$ umbellatarum Nees (Agathis) $* \rightarrow 22$ umbellina Nixon (Exotela) $* \rightarrow 304$ umbraculator Nees (Iphiaulax) $* \rightarrow 667$ umbrata Stelfox (Alysia) $* \rightarrow 65$ umbratilis Haliday (Clinocentrus) $* \rightarrow 948$ umbratus Trentepohl (Spathius) $\rightarrow 788$ uncigenis Wesmael (Eubazus) $\rightarrow 507$ undulatus Ratzeburg (Doryctes) $* \rightarrow 758$ ungularis Thomson (Coeloides) $* \rightarrow 659$ ungularis Thomson (Phaenocarpa) $* 346$ unicolor Enderlein (Homolobus) $\rightarrow 1014$ unicolor sensu Ruthe (Meteorus) $\rightarrow 873$ unicolor Wesmael (Aleiodes) $\rightarrow 1472$ unicolor Wesmael (Meteorus) $\rightarrow 876$ unicolot Wesmael (Petalodes) $\rightarrow 1484$ unilachni Gahan (Pauesia) $* \rightarrow 450$ unipunctator Thunberg (Aleiodes) $* \rightarrow 1482$ urinator Fabricius (Bracon) * $\rightarrow 644$ urticae Haliday (Aphidius) $* \rightarrow 395$ urticae Mackauer (Misaphidus) $\rightarrow 426$ ustulata Fahringer (Ontsira) $\rightarrow 773$ utilis Muesebeck (Trioxys) $\rightarrow 469$ uzbekistanicus Luzhetzki (Aphidius) $* \rightarrow 396$ vaenia Nixon (Antrusa) $* \rightarrow 70$ vagans Ruthe (Blacus) $\rightarrow 796$ vagator Förster (Biosteres) $\rightarrow 1277$ vaginator Westmael (Rilipertus) $* \rightarrow 915$ vagus Ruthe (Eubazus) * $\rightarrow 508$ valentinus Quilis (Aphidius) $\rightarrow 385$ validus Haliday (Ephedrus) $* \rightarrow 415$ vanessae Reinhard (Cotesia) $* \rightarrow 1110$ variabilis Herrich-Schäffer (Chelonus) $\rightarrow 707$ variabilis Szépligeti (Rhysipolis) $\rightarrow 977$ variator Nees (Bracon) $* \rightarrow 645$ varicoxa Thomson (Rhysipolis) $\rightarrow 977$ variegata Boheman (Pseudoripio) $\rightarrow 671$ variegator Spinola (Bracon) $* \rightarrow 646$ variegator Szépligeti (Misaphidus) $\rightarrow 431$ variegatus Szépligeti (Opius) * $\rightarrow 1400$ varipes Ruthe (Microplitis) $* \rightarrow 1242$ varipes Szépligeti (Opius) $\rightarrow 1350$ varipes Thomson (Agathis) $* \rightarrow 23$ varipes Wesmael (Ascogaster) $* \rightarrow 698$ varius Herrich-Schäffer (Aleiodes) $* \rightarrow 1483$ varius Nees (Pauesia) $\rightarrow 446$ venatrix Marshall (Mesocrina) $\rightarrow 313$ venusta Haliday (Dinotrema) $\rightarrow 288$ venustus Telenga (Bracon) $\rightarrow 609$ venustus Tobias (Chorebus) $* \rightarrow 211$ veratri Griffiths (Chorebus) $* \rightarrow 212$ vernalis Wesmael (Syntretus) $\rightarrow 923$ versicolor Wesmael (Meteorus) $* \rightarrow 880$ vestalis Haliday (Cotesia) $* \rightarrow 1111$ vestigator Haliday (Clinocentrus) $* \rightarrow 949$ vexator Fischer (Opius) $\rightarrow 1377$ viaticus Sedlag (Lysaphidus) $* \rightarrow 419$ viator Förster (Adelius) $* \rightarrow 9$ victoris Telenga (Bassus) $\rightarrow 39$ victus Haliday (Opius) $* \rightarrow 1401$ vidalis Quilis (Ephedrus) $\rightarrow 413$ vidua Curtis (Coelinidea) $* \rightarrow 222$ viduus Ruthe (Microplitis) $* \rightarrow 1243$ viennensis Giraud (Neoneurus) $\rightarrow 883$ vigilax Kokujev (Bracon) $\rightarrow 590$ villana Reinhard (Cotesia) $* \rightarrow 1112$ viminetorum Wesmael (Pholetesor) $* \rightarrow 1255$ vinimetorum Lyle (Dolichogenidea) $\rightarrow 1145$ vinulae Bouché (Cotesia) $\rightarrow 1063$ vipio Reinhard (Napamus) $* \rightarrow 1245$ viridanae Johannson (Meteorus) $\rightarrow 870$ vitripennis Curtis (Glyptapanteles) $* \rightarrow 1176$ vitripennis Herrich-Schäffer (Eubazus) $* 509$ vitripennis Ratzeburg (Bracon) $* \rightarrow 647$ vitripennis Telenga (Aleiodes) $\rightarrow 1455$ vittatae Muesebeck (Perilitus) $* \rightarrow 909$ vittatus Ruschka (Opius) $\rightarrow 1364$ vittiger Wesmael (Aleiodes) $\rightarrow 1479$ vividus Papp (Colastes) $* \rightarrow 964$ vocketothi Fischer (Opius) $\rightarrow 1311$ voloscensis Fischer (Meteorus) $\rightarrow 851$ volucre Haliday (Praon) $* \rightarrow 463$ vulgaris Bouché ? (Diaeretiella) $\rightarrow 404$ 
vulgaris Cresson (Meteorus) $\rightarrow 875$

vulgaris Ruthe (Microgaster) $\rightarrow 1198$

wesmaeli Bengtsson (Homolobus) $\rightarrow 1013$

wesmaeli Boie (Zele) $\rightarrow 936$

wesmaeli Haliday misspell. (Biosteres) $\rightarrow 1293$

wesmacli Ruthe (Paroplitis) $* \rightarrow 1246$

wesmaelii Haliday (Biosteres) $* \rightarrow 1293$

wesmaclii Ratzeburg (Microtypus) $* \rightarrow 1417$

westphalica Königsmann (Idiasta) $* \rightarrow 310$

wissmannii Ratzeburg (Protaphidius) $* 464$

woerziphagus Fischer (Opius) $* \rightarrow 1402$

wollastonii Cabrera (Misaphidus) $\rightarrow 427$

xanthocephalus Marshall (Syntretus) $* \rightarrow 932$

xanthogaster Nees (Bracon) $* \rightarrow 648$

xanthomelas Wesmael (Meteorus) $\rightarrow 863$

xanthopus Förster (Diachasma) $* \rightarrow 1300$

xanthopus Ruthe (Microplitis) * $\rightarrow 1244$ xanthostigma Haliday (Apanteles) $* \rightarrow 1060$

xiphidius Griffiths (Chorebus) $* \rightarrow 213$

xiphydriae Tobias (Eubazus) $\rightarrow 496$

xylostei Marshall (Opius) $\rightarrow 1389$

xylostellus Griffiths (Chorebus) $* \rightarrow 214$

zaykovi Nixon (Bassus) $* \rightarrow 40$

zelotes Marshall (Utetes) $* \rightarrow 1415$

zernyanus Fahringer (Ascogaster) $\rightarrow 687$

zimini Tobias (Chelonus) $* \rightarrow 719$

ziratus Papp (Desmiostoma) $\rightarrow 1296$

zonator Szépligeti (Kerorgilus) $\rightarrow^{*}$ record uncertain zonatus Marshall (Bassus) $\rightarrow 29$

zonatus Wesmael (Ontsira) $\rightarrow 772$

zujlenivan Achterberg\& Haeselbarth (Syntretus) $* \rightarrow 933$

zygaenae Nees (Aleiodes) $\rightarrow 1454$

zygaenarum Marshall (Cotesia) $* \rightarrow 1113$

\section{Acknowledgements}

We wish to thank Dr M. SHAW (Edinburgh) and Mr J. W. A. VAN ZUtJLEN (Waalwijk) for providing information on several listed species, and Mr. A. D. LISTON (Frontenhausen) for checking the English of the manuscript.

\section{Author's addresses:}

S. A. BELOKOBYLSKI, Zoological Institute, Russian Academy of Sciences Universitetskaya nab. 1 St. Petersburg 199034 Russia

/ Museum i Institut zoologii PAN, Wilcza 64, Warsaw, Poland,e-mail: hymenopt@zin.ru

Dr. A. TAEgER

Deutsches Entomologisches Institut im Zentrum für Agrarlandschafts- und Landnutzungsforschung (ZALF), Schicklerstr. 5, D-16225 Eberswalde, Germany, e-mail: taeger@zalf.de

\section{VAN ACHTERBERG}

Department of Entomology

Nationaal Natuurhistorisch Museum

Postbus 9517, 2300 RA Leiden, Netherlands

e-mail: achterberg@naturalis.nnm.nl

E. HAESELBARTH

c/o Zoologische Staatssammlung

Münchhausenstr. 21, D- 81247 München

M. RIEDEL, Am Hamberg 8

D-29683 Bad Fallingborstel

e-mail:mamaflo.Riedel@t-online.de 